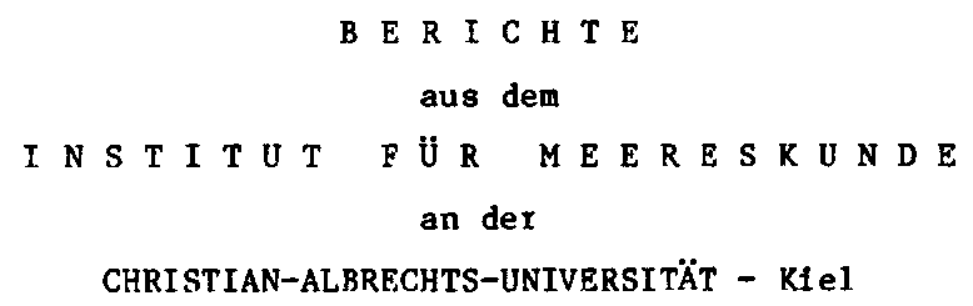

$\mathrm{Nr} \cdot 132$

1984

I S O P Y C A I C A T LA S

$$
\text { O } \mathbf{P} \text { T } \mathbf{H} \mathbf{E}
$$

RORTA A T LA T T C OC BA A

- monthly wean maps and sections -

by

J. Bauer and J.D. Woods

\title{
DO1 10.3289/IFM_BER_132
}

Copies are available from

Institut für Meereskunde an der Universitüt

Abt. Regionale Ozeanographie

Disternbrooker Weg 20

D-2300 Kiel 1, FRG 
1. INTRODUCTION 1

2. DATA PROCESSING 3

2.1 The Robinson-Bauer-Schroeder numerical at las 3

2.2 Seasonal variation 3

2.3 Density profiles 5

2.4 Isopycnals 5

3. THE NUMERICAL ATLAS ON THE IFM COMPOTER 6

3.1 General data structure 6

3.2 Processing programs 6

4. REFERENCES 8

5. ACKOOWLDGEMENTS 10

6. FIGURES 11

6.1 List of figures 11

6.2 Figures $(1-160) \quad 15$ 


\section{INTRODUCTION}

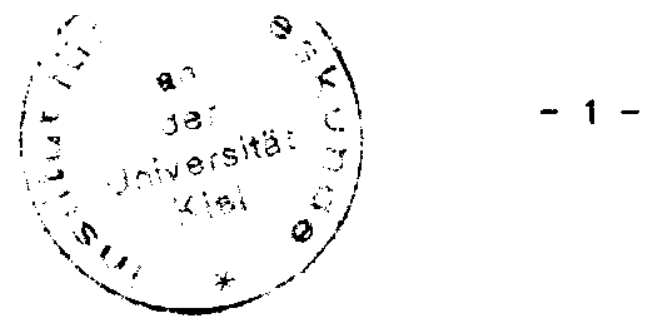

The distributions presented in this volume have been dexived from the well-known Robinson-Bauer-Schroeder (1979) analysis of bathythermograph and hydrocast archive data to give monthly mean temperature and mean salinity values at standard depths in a $1^{\circ} \times 1^{\circ}$ array. Their analyzed data have been interpolated onto density surfaces and are presented as isopycnic maps and sections. The analysis was undertaken as part of an investigation of water mass conversion and circulation in the warm water sphere of the North Atlantic Ocean.

Isopycnic analysis was Introduced into oceanography fifty years ago by Montgomery (1938) and Parr (1938). Isopycnic maps and sections have since appeared in many atlases and papers, although the traditional isobaric presentation remains much more common. A selection of publications including isopycnic maps or sections is listed on page 3 .

Presentation of hydrographic data as distributions on 1sopycnic surfaces offers a number of advantages over the more common isobaric presentation. Firstly, mixing occurs more freely along density surfaces than across them because the former involves no work againgt the Archimedes force. The power input to three dimensional turbulence in the stratified interior of the ocean is so weak that diapycnic wixing normally achieves very much less vertical transport of passive scalars than 1sopycnic mixing along inclined (baroclinic) density surfaces by quasi-geostrophic turbulence (Garrett, 1982).

$$
\text { 1.e. } k_{d} \nabla_{d} s \ll b^{2} \cdot k_{1} \nabla_{1} s
$$

where the subscripts $d$ and $i$ refer to components of the diffusivities $K$ and the gradients $\nabla$ directed across and along the density surface respectively, and $b$ is the slope of the density surface. For that reason, isopycnic analysis has proved particularly valuable in geochemistry (Broecker, 1981) and especially in studying the spreading of transient tracers (Sarmiento et al., 1982).

Isopycnic analysis is also the first step in mapping the distribution of potential vorticity, which is inversely proportional to the spacing between two isopycnic surfaces. Maps of thickness have been published by Tsuchiya (1968). Potential vorticity is mixed along density surfaces like Rhines, 1979. Maps of the large-scale isopycnic distribution of Sverdrupian potential vorticity (in which the relative vorticity is assumed to be negligible compared with the planetary vorticity because of the large scale) have been 
published by Sarmiento (1982) and McDowe11, Rhines \& Keffer, 1982. Mesoscale isopycnic maps of thickness and potential vorticity have been published by Leach, Minnett \& Woods (1984) and Fischer, Leach \& Woods (1985).

The analysis of climatological data onto isopycnic (rather than isobaric) surfaces also factlitates comparison with expedition data, since it eliminates spuxious differences arising from transient vertical displacement by internal waves and quasi-geostrophic eddies (see, for example, the comparison in Leach, Minnett \& Woods, 1985).

Some publications that include Isopycnic aps or sections

\begin{tabular}{|c|c|c|}
\hline Date & Authors & Reference \\
\hline 1937 & R.B. Montgomery & Bul1. American Met. Soc. 18, 210-212 \\
\hline 1938 & E.A. Parr & J. Max. Res. 1, 269-290 \\
\hline 1942 & $\begin{array}{l}\text { R.B. Montgomery } \\
\text { \& M.J. Pollak }\end{array}$ & J. Mar. Res. 5, 20-27 \\
\hline 1965 & J.L. Reld & Internediate waters of the Pacific Oean \\
\hline 1968 & M. Tsuchiya & Upper waters of the Intertropical Pacific Oc. \\
\hline 1968 & R.A. Barkley & Oceanographic Atlas of the Pacific Ocean \\
\hline $1970-73$ & C.M. Love (Ed.) & Bastropac atlas (7 volumes) \\
\hline 1971 & R. Wyrtki & $\begin{array}{l}\text { Oceanographic atlas of the International } \\
\text { Indian Ocean expedition }\end{array}$ \\
\hline 1971 & $\begin{array}{l}\text { J.L. Retd \& } \\
\text { R.J. Lynn }\end{array}$ & Deep-Sea Res. 18, 1063-1088 \\
\hline 1972 & J.E. Callahan & Deep-Sea Res. 19, 563-575 \\
\hline 1973 & J.L. Reid & Northwest Paciffc ocean waters in winter \\
\hline 1981 & $\begin{array}{l}\text { A.L. Gordon \& } \\
\text { B.J. Molinelil }\end{array}$ & Southern Ocean Atlas \\
\hline 1982 & S. Levitus & Climatological Atlas of the Dorld Ocean \\
\hline 1982 & $\begin{array}{l}\text { J.L. Sarmiento } \\
\text { C.G.H. Rooth } \\
\text { \&. Roether }\end{array}$ & J. Geophys. Res. 87, 8047-8056. \\
\hline
\end{tabular}




\section{DATA PROCESSING}

\subsection{The Robinson-Bauer-Schroeder numerical atlas}

The starting point for our work was the numerical version of the atlas produced by Robinson, Bauer \& Schroeder (1979). Descriptions of the archive data used in the compllation of the atlas, and the processing method, are given in Robinson, Bauer \& Schroeder (1979). Its spectal feature is that the concentration of bathythermograph data is sufficient to justify calculation of monthly mean temperature at intervals of $1^{\circ}$ latitude and long 1 tude in the top $150 \mathrm{~m}$. The high spatial resolution has been retained for temperature at depths greater than $150 \mathrm{~m}$ and for salinity at all depths, although the data base is then too small to justify stratification by month of the year. The monthly mean temperatures at depths down to $150 \mathrm{~m}$ and the high spatial resolution provide information about the seasonal cycle that is missing missing from atlases (e.g. Levitus, 1982) which use hydrocast, but not bathythermograph data.

\subsection{Seasonal variation}

The Robinson-Bauer-Schroeder numerical atlas offers us the possibility of climatological isopycnic analysis of the seasonally varying component of the upper ocean. On the other hand the limitations of the atlas (no monthly mean temperatures below 150 on or salinities at any depth) forced us to base our analysis on a pseudo-monthly-mean density, which is only an approximation to the monthly mean density that would be calculated from an "ideal" atlas comprising monthly mean temperature and salinity at all depths. Our assumption is that the difference between the pseudo- and Ideal-monthly mean density distributions are not so serious as to invalidate the isopycnic distributions presented herein. Differences can arise from a variety of sources. We shall briefly consider three of them, namely (1) seasonal vartation below $150 \mathrm{~m}$, (2) seasonal variation of salinity in the top 150 m and (3) secular change of temperature and salinity. 


\section{(1) Seasonal variation below 150 metres}

The numerical at las data exhibit significant variation of temperature at $150 \mathrm{~m}$, the maximum depth for which monthly mean values are given. An estimate of the maximum depth of seasonal variation might be obtained by determining the depth at which the seasonal minimum surface temperature occurs in the annual mean temperature profile. Robinson et al . (1979) used a similar method, based on the monthly mean surface temperature minus 2 Fahrenhelt degrees (approximately $1 \mathrm{~K}$ ), to determine the depth of the mixed layer each month. Only in or near the tropics is the annual maximum depth of the mixed layer, so calculated, less than $150 \mathrm{~m}$. Seasonal variation extends deeper at higher latitudes.

\section{(2) Seasonal variation of salinity}

Seasonal varfation of salinity at North Atlantic Ocean Weather Stations has been described by Taylor S Stephens (1980). Table 1 shows the range of surface density variations due to the seasonal cycle of salfnity. The changes are small and contribute little to the seasonal variation of density in the seasonal pycnocline. If there were no seasonal variation of salintty, isohalines would remain vertical in an isopycnally plotted section through the seasonal pycnocline. The isohalines in fig. 1 do indeed rise almost vertically through the seasonal pycnocline, showing that neglect of the seasonal variation of salinity will not normally lead to serious error in calculation of the depths of isopycnals in the seasonal pycnocllne. The neglect of seasonal variation of salinity in our analysis and the data bias towards summer samples do not therefore seem to be serious.

Table 1: Annual range of surface density due to salinity changes observed at Ocean Weather Stations (after Taylor \& Stephens, 1980)

\begin{tabular}{|c|c|c|c|c|c|}
\hline$B \Delta S / \mathrm{kg} \mathrm{m}^{-3}$ & ows & Latitud & & Longit & ude \\
\hline $\begin{array}{l}0.048 \\
0.143 \\
0.080 \\
0.177 \\
0.070 \\
0.040 \\
0.028 \\
0.021 \\
0.068\end{array}$ & $\begin{array}{l}\text { A } \\
\text { B } \\
\text { C } \\
\text { D } \\
\text { B } \\
\text { I } \\
\text { J } \\
\text { R } \\
\text { M }\end{array}$ & $\begin{array}{l}62^{\circ} \\
56.5^{\circ} \\
52.7^{\circ} \\
44^{\circ} \\
35^{\circ} \\
59^{\circ} \\
52.5 \\
45^{\circ} \\
66^{\circ}\end{array}$ & $\begin{array}{l}N \\
N \\
N \\
N \\
N \\
N \\
N \\
N \\
N\end{array}$ & $\begin{array}{l}33^{\circ} \\
51^{\circ} \\
35.5^{\circ} \\
41^{\circ} \\
48^{\circ} \\
19^{\circ} \\
20^{\circ} \\
16^{\circ} \\
2^{\circ}\end{array}$ & $\begin{array}{l}W \\
W \\
W \\
W \\
W \\
W \\
W \\
W \\
E\end{array}$ \\
\hline
\end{tabular}


(3) Longer term changes of temperature and salintty

Pu \& Pollard (1984) have shown that the salfitty in the North East Atlantic has changed significantly over recent decades. There is also evidence of interannual and decadal variation of temperature. The archive data set sampled this secular variation unevenly over the period 1900 to 1974 , with different sampling profiles for temperature and salinity in the upper $150 \mathrm{~m}$ because the former is dominated by bathythermographs and the latter by hydrocasts. Below $150 \mathrm{~m}$, both temperature and salinfty were obtained from hydrocast data with the same sampling profile. Our knowledge concerning interannual and decadal variation is not sufficient to fustify attempting to correct for these sampling biases.

\subsection{Density profiles}

The monthly mean density profiles were calculated for the whole water column, from the atlas temperature and salinity data. Density inversions were elfminated by monotonisation wherever they occurred, above $150 \mathrm{~m} \mathrm{In}$ the winter months. Wherever density values at depths greater than $150 \mathrm{~m}$ made inversions, they were replaced with the value at $150 \mathrm{~m}(\mathrm{fig} .2)$. Temperature was corrected to the value consistent with salinity and density where this correction was made.

\subsection{Isopyenals}

Values of pressure, temperature and salinity were interpolated vertically onto selected density surfaces. 


\section{THE MUMERICAL ATLAS ON THE IFM COMPUTER}

This chapter gives a brief introduction to using the numerical atlas installed on the computer of the Inst1tut für Meereskunde (VAX 11/750). It describes the general structure of the data, and the programs used to extract the products illustrated in this atlas.

\subsection{General data structure}

The numerical at las installed at the IfM computer contains hydrographical data of the Atlantic Ocean between $5^{\circ} \mathrm{S}$ and $60^{\circ} \mathrm{N}$ and $0^{\circ} \mathrm{W}$ and $90^{\circ} \mathrm{W}$ in a $1^{\circ} \times 1^{\circ}$ geographical grid and at the following depth levels: $0,30,60,90$, $125,150,183,200,244,250,300,305,366,400,500,600,700,800,900$, $1000,1100,1200,1300,1400,1500,1750,2000,2500,3000,4000,5000$, $6000,7000 \mathrm{~m}$. The basic data set contains 4266 profiles recorded in 112074 cycles of 17 variables.

It starts at the north-west corner of the North Atlantic. Along each lattude profiles are aligned from west to east. The mean value calculated by averaging the sample values lying in a $1^{\circ} \times 1^{\circ}$ gxid square is labeled to the centre of the grid square $(f 1 g$. 3). Position values are positive north of the equator and east of the Greenwich meridian and negative south of the equator and west of Greemolch. The data 18 stored In the Mik4-Forwat (Holtorff, 1980), a binary code for real numbers, used at the IFM for all $k$ inds of data processing. It 18 therefore compatible with other data used at the IFM. The standard processing programes of the MR-Sygtea could be applied for further processing of the atlas data and vartous transfer programs allow an uncomplicated transfer to other computers (Holtorff, 1984 ).

\subsection{Proceseing prograns}

The computer programs used to obtain the products presented in this volume from the numerical atlas are briefly described below.

Various kinds of subsets can be extracted from a data set by program ExTRC. It was applied here to select monthiy files in profile form from the atlas data set.

$\sigma_{\theta}$ was calculated from wonthly mean temperatuxes and all-data mean balinities using program EICAS. 
Program MONINT monotonized the density, reiterated temperature and interpolated temperature, salinfty and depth on selected $\sigma_{\theta}$ values.

Again EXTRAC selected single surfaces of constant $\sigma_{\theta}$ out of the Interpolated profiles.

Program MEGRUM a two-dimensional interpolation contour program for geographical data fields was used to produce the horizontal charts. The positions of the isolines are converted into geographical coordinates.

The plotting of the charts was done with PLMEGR, which plots a cartographic projection on a cylinder mantle (e.g. Mexcator projection). The possibility to include data sets with geographical coordinates was used here to plot coastilnes and isolines.

The objective analysis program OBJANT was applied to produce equally spaced profiles along the tracks of the SFB standard sections.

The contouring of the vertical sections was done with program CONREC. 


\section{REFERENCES}

Baxkley, R.A., 1968: Oceanographtc Atlas of the Paciflc Ocean, University of Hawaif Press, Honolulu.

Broecker, W.S., 1981: Geochemical tracers and ocean c1 rculation. In "Evolution of physical oceanography", edited by B. Warren \& $C$. Wunsch MIT Press, Cambridge, Mass., pp. 434-460.

Callahan, J.E., 1972: The structure and circulation of deep water in the Antarctic. Deep-Sea Res., 19, 563-575.

Fischer, J., H. Leach \& J.D. Woods, 1985: Components of Upper Ocean Potential Vortfity at the North Atlantic Polar Front.

(In preparation).

Garrett, C., 1982: On the parameterization of diapycnal fluxes due to doublediffusive intrusions.

J. Phys. Oceanogr. 12(9), 953-959.

Gordon, A.L. \& E.J. Molinel1i, 1981: Southern Ocean Atlas. Pt. 1. Thermoand Chemical Distributions. Columbla UnIversity Press, N.Y.

Holtorff, J., 1980: MK4-Documentation.

Dept. of Theoretical Oceanography, Institut für Meereskunde, K1el.

Holtorff, J., 1984: Program Library of the Inst. f. Meeresk. (unpublished) Dept. of Theoretical Oceanography, Institut für Meereskunde, Kiel.

Leach, H., P.J. Minnett \& J.D. Woods, 1985: Deep-Sea Res. (in press)

Levitus, S., 1982: Climatological Atlas of the World Ocean. NOAA Professional Paper 13, U.S. Dept. of Commerce, National Oceantc and Atmospheric Administration, 173 pp.

Love, C.M. (Editor) 1970-1973: EASTROPAC Atlas (7 volumes). U.S. Dept. of Commerce.

McDowel1, S., P. Rhines \& T. Keffer, 1982: North Atlantic potent1al vorticity and its relation to the general circulation.

J. Phys. Oceanogr. 12, 1417-1436.

Meincke, J. \& A. Sy, 1983: Large-scale effects of the Mid-Atlant1c Ridge on the North Atlantic Current. ICES Hydrography Comnd tee, C.M. 1983/C:8, 10 pp.

Montgomery, R.B., 1937: A suggested method for represent1ng gradient, flow in isentropic surfaces.

Bu11. Amer. Met. Soc. 18(6-7): 210-212. 
Montgomery, R. B., 1938: Circulation in Upper Layers of Southern North Atlantic Deduced with Use of Isentroptc Analysis.

Papers in Phystcal Oceanography and Meteorology, MIT and WHOI, 6(2), 55 pp.

Montgomery, R.B. \& M.J. Pollack, 1942: Sigma-T surfaces in the Atlantic ocean.

J. Mar. Res. 5, 20-27.

Parr, A.E., 1938: Analysis of current profiles by a study of pycnomeric distortion and identifying properties.

J. Mar. Res. 1, 269-290.

Pu, S. \& R. Pollard, 1984: Structure and ventilation of the upper Atlantic Ocean northeast of the Azores.

Ms. 2nd draft 27.1.84.

Reid, J.L., 1965: Intermediate waters of the Pacific Ocean.

The Johns Hopkins Press, Baltimore.

Reid, J.L. \& R.J. Lynn, 1971: On the Influence of the Norwegian-Greenland and Weddell Seas upon the Bottom Waters of the Indian and Pacific Oceans. Deep-Sea Res. 18, 1063-1088.

Re1d, J.L., 1973: Northwest Pacific Ocean Waters in Winter. The Johns Hopkins Press, Baltimore.

Rhines, P.B., 1979: Geostrophic Turbulence. Ann. Rev. Fluid Mech. 11, 401-441.

Robinson, M., R. Bauex \& E. Schroeder, 1979: Atlas of North Atlantic - Indian Ocean Monthy Mean Temperatures and Mean Salinities of the Surface Layer.

Dept. of the Navy, Washington, D.C.

Sarmiento, J.L., 1982: Time scales of thermocline exchange with surface waters.

Unpublished manuscript.

Sarmiento, J.L., C.G.H. Rooth \& W. Roether, 1982: The North Atlantic Tritfum Distribution in 1972 .

J. Geophys. Res. 87(C10), 8047-8056.

Taylor, A.H. \& J.A. Stephens, 1980: Seasonal and Year-to-year Variations in Surface Salinity at the nine North Atlantic Ocean Weather Stations. Oceanologica Acta, $3(4), 421-430$.

Tsuch1ya, M., 1968: Upper waters of the intertropical Pacific Ocean. The Johns Hopkins Press, Baltimore.

Wyrtk1, K., 1971: Oceanographic Atlas of the International Indian Ocean Expedition.

National Science Foundation, Washington, U.S.A. 


\section{ACKNOWLEDGEMENTS}

This work was supported by the Deutsche Forschungsgemeinschaft (German Research Society) under contract DFG-SFB-133 as part of the collaborative research program "Warm water sphere of the Atlantlc" at the Institut für Meereskunde, Christian-Al brechts-Universität, Kiel.

The data set we used is the numerical atlas produced by M. Robinson, R. Bauer and E. Schroeder (1979), which we bought frow Compass System Inc., 4640 Jewell St. \#204, San D1ego, CA. 92109, USA.

We thank Detlef Stammer for help with the computer plots and Alfred Eisele for the cartography. 


\section{FIGURES}

\subsection{List of figures}

Explaining examples

Fig. 1: Salinity section in isopycnic presentation 15

Fig. 2: February and September mean profiles at $50^{\circ} \mathrm{N}, 35^{\circ} \mathrm{W} \quad 16$

Fig. 3: Horizontal and vertical positioning of atlas data 16

\section{Monthly mean waps}

Fig. 4: Pressure on $\sigma_{\theta}=25.0 \mathrm{~kg} \mathrm{~m} \mathrm{~m}^{-3}$ - January 17

Fig. 5: Temperature on $\sigma_{\theta}=25.0 \mathrm{~kg} \mathrm{~m}-3$ January 18

Fig. 6: Salinity on $\sigma_{\theta}=25.0 \mathrm{~kg} \mathrm{~m}^{-3}$ - January 19

Fig. 7: Pressure on $\sigma_{\theta}=26.0 \mathrm{~kg} \mathrm{~m}-3$ - January 20

Fig. 8: Temperature on $\sigma_{\theta}=26.0 \mathrm{~kg} \mathrm{~m}-3$ January 21

Fig. 9: Salinity on $\sigma_{\theta}=26.0 \mathrm{~kg} \mathrm{~m}^{-3}$ - January 22

Fig. 10: Pressure on $\sigma_{\theta}=27.0 \mathrm{~kg} \mathrm{~m}^{-3}-$ January 23

Fig. 11: Temperature on $\sigma_{\theta}=27.0 \mathrm{~kg} \mathrm{~m}^{-3}$ - January 24

Fig. 12: Salinity on $\sigma_{\theta}=27.0 \mathrm{~kg} \mathrm{~m}^{-3}$ - January 25

F1g. 13: Pressure on $\sigma_{\theta}=25.0 \mathrm{~kg} \mathrm{~m}^{-3}$ - February 26

Fig. 14: Temperature on $\sigma_{\theta}=25.0 \mathrm{~kg} \mathrm{~m}^{-3}$ - February 27

Fig. 15: Salinity on $\sigma_{\theta}=25.0 \mathrm{~kg} \mathrm{~m}^{-3}-$ February 28

Fig. 16: Pressure on $\sigma_{\theta}=26.0 \mathrm{~kg} \mathrm{~m}-3$ - February 29

Fig. 17: Temperature on $\sigma_{\theta}=26.0 \mathrm{~kg} \mathrm{~m}-3$ - February 30

Fig. 18: Salinity on $\sigma_{\theta}=26.0 \mathrm{~kg} \pi^{-3}$ - February 31

Fig. 19: Pressure on $\sigma_{\theta}=27.0 \mathrm{~kg} \mathrm{~m}-3$ - February 32

Fig. 20: Temperature on $\sigma_{\theta}=27.0 \mathrm{~kg} \mathrm{~m}^{-3}-$ February 33

Fig. 21: Salinity on $\sigma_{\theta}=27.0 \mathrm{~kg} \mathrm{~m}^{-3}$ - February 34

F1g. 22: Pressure on $\sigma_{\theta}=27.5 \mathrm{~kg} \mathrm{~m}^{-3}$ - February 35

Fig. 23: Temperature on $\sigma_{\theta}=27.5 \mathrm{~kg} \mathrm{~m}^{-3}$ - February 36

F1g. 24: Salinity on $\sigma_{\theta}=27.5 \mathrm{~kg} \mathrm{\textrm {m } ^ { - 3 }}$ - February 37

F1g. 25: Pressure on $\sigma_{\theta}=25.0 \mathrm{~kg} \mathrm{~m}^{-3}-$ March 38

Fig. 26: Temperature on $\sigma_{\theta}=25.0 \mathrm{~kg} \mathrm{~m}^{-3}-$ March 39

Fig. 27: Salinity on $\sigma_{\theta}=25.0 \mathrm{~kg} \mathrm{~m}-3$ March 40

F1g. 28: Pressure on $\sigma_{\theta}=26.0 \mathrm{~kg} \mathrm{~m}^{-3}-$ March 41

Fig. 29: Temperature on $\sigma_{\theta}=26.0 \mathrm{~kg} \mathrm{~m}-3$ March 42

F1g. 30: Salinity on $\sigma_{\theta}=26.0 \mathrm{~kg} \mathrm{w-3}$ - March

Fig. 31: Pressure on $\sigma_{\theta}=27.0 \mathrm{~kg} \mathrm{~m}-3$-March 44

F1g. 32: Temperature on $\sigma_{\theta}=27.0 \mathrm{~kg} \mathrm{~m}-3$ March 45

FIg. 33: Salinity on $\sigma_{\theta}=27.0 \mathrm{~kg} \mathrm{~m}-3$ March 46

Fig. 34: Pressure on $\sigma_{\theta}=27.5 \mathrm{~kg} \mathrm{~m}^{-3}$ - March 47

Fig. 35: Temperature on $\sigma_{\theta}=27.5 \mathrm{~kg} \mathrm{w}^{-3}$ - March 48

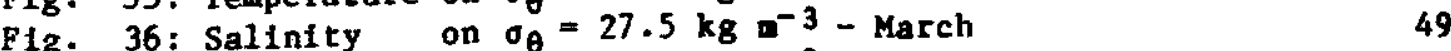

Fig. 37: Pressure on $\sigma_{\theta}=25.0 \mathrm{~kg} \mathrm{~m}^{-3}$ - April

F18. 38: Temperature on $\sigma_{\theta}=25.0 \mathrm{~kg} \mathbf{m}^{-3}-$ Apr11

Fig. 39: Salinfty on $\sigma_{\theta}=25.0 \mathrm{~kg} \mathrm{m-3}-$ April

F1g. 40: Pressure on $\sigma_{\theta}=26.0 \mathrm{~kg} \mathrm{m-3}$ - Apri1

Fig. 41: Temperature on $\sigma_{\theta}=26.0 \mathrm{~kg} \mathrm{~m}-3-$ Apri1

Fig. 42: Salinity on $\sigma_{\theta}=26.0 \mathrm{~kg} \mathrm{~m}-3$ Apri1

FIg. 43: Pressure on $\sigma_{\theta}=27.0 \mathrm{~kg} \mathrm{~m}-3-A p r 11 \quad 56$

F1g. 44: Temperature on $\sigma_{\theta}=27.0 \mathrm{~kg} \mathrm{~m}-3-A p r 11 \quad 57$

Fig. 45: Salinity on $\sigma_{\theta}=27.0 \mathrm{~kg} \mathrm{\textrm {m } ^ { - 3 } - \text { April }} 58$ 
Page

Fig. 46: Pressure on $\sigma_{\theta}=25.0 \mathrm{~kg} \mathrm{~m}^{-3}-$ May 59

Fig. 47: Temperature on $\sigma_{\theta}=25.0 \mathrm{~kg} \mathrm{~m}^{-3}-$ May 60

Fig. 48: Salinity on $\sigma_{\theta}=25.0 \mathrm{~kg} \mathrm{~m}^{-3}-$ May 61

Fig. 49: Pressure on $\sigma_{\theta}=26.0 \mathrm{~kg} \mathrm{~m}^{-3}-$ May 62

Fig. 50: Temperature on $\sigma_{\theta}=26.0 \mathrm{~kg} \mathrm{~m}-3$ - May 63

Ffg. S1: Salinity on $\sigma_{\theta}=26.0 \mathrm{~kg} \mathrm{~m}^{-3}-$ May 64

Fig. 52: Pressure on $\sigma_{\theta}=27.0 \mathrm{~kg} \mathrm{~m}^{-3}-$ May 65

Fig. 53: Temperature on $\sigma_{\theta}=27.0 \mathrm{~kg} \mathrm{~m}-3$ - May 66

Fig. 54: Salinity on $\sigma_{\theta}=27.0 \mathrm{~kg} \mathrm{~m}^{-3}-$ May $\quad 67$

Fig. 55: Pressure on $\sigma_{\theta}=25.0 \mathrm{~kg} \mathrm{~m}^{-3}$ - June 68

Fig. $56:$ Temperature on $\sigma_{\theta}=25.0 \mathrm{~kg} \mathrm{~m}^{-3}$ - June 69

Fig. 57: Salinity on $\sigma_{\theta}=25.0 \mathrm{~kg} \mathrm{\textrm {m } ^ { - 3 } - J u n e} 70$

Fig. 58: Pressure on $\sigma_{\theta}=25.5 \mathrm{~kg} \mathrm{~m}^{-3}-$ June 71

Fig. 59: Teraperature on $\sigma_{\theta}=25.5 \mathrm{~kg} \mathrm{~m}^{-3}$ - June 72

Fig. 60: Salinity on $\sigma_{\theta}=25.5 \mathrm{~kg} \mathrm{~m}^{-3}$ - June 73

Fig. 61: Pressure on $\sigma_{\theta}=26.0 \mathrm{~kg} \mathrm{~m}^{-3}-J u n e \quad 74$

Fig. 62: Temperature on $\sigma_{\theta}=26.0 \mathrm{~kg} \mathrm{~m}^{-3}-$ June 75

Fig. 63: Salinity on $\sigma_{\theta}=26.0 \mathrm{~kg} \mathrm{~m}^{-3}-$ June 76

Fig. 64: Pressure on $\sigma_{\theta}=26.5 \mathrm{~kg} \mathrm{~m}^{-3}$ - June 77

Fig. 65: Temperature on $\sigma_{\theta}=26.5 \mathrm{~kg} \mathrm{~m}^{-3}-$ June 78

Fig. 66: Salinity on $\sigma_{\theta}=26.5 \mathrm{~kg} \mathrm{~m}^{-3}$ - June 79

Fig. 67: Pressure on $\sigma_{\theta}=27.0 \mathrm{~kg} \mathrm{~m}^{-3}-$ June 80

Fig. 68: Temperature on $\sigma_{\theta}=27.0 \mathrm{~kg} \mathrm{~m}-3$ June 81

Fig. 69: Salinity on $\sigma_{\theta}=27.0 \mathrm{~kg} \mathrm{~m}^{-3}$ - June 82

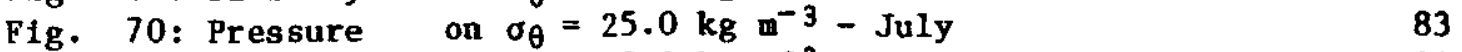

Fig. 71: Temperature on $\sigma_{\theta}=25.0 \mathrm{~kg} \mathrm{~m}^{-3}-\mathrm{July} \quad 84$

Fig. 72: Salinity on $\sigma_{\theta}=25.0 \mathrm{~kg} \mathrm{~m}^{-3}-\mathrm{July} \quad 85$

Fig. 73: Pressure on $\sigma_{\theta}=25.5 \mathrm{~kg} \mathrm{~m}-3$ July 86

Fig. 74: Temperature on $\sigma_{\theta}=25.5 \mathrm{~kg} \mathrm{~m}^{-3}-\mathrm{July} \quad 87$

Fig. 75: Salinity on $\sigma_{\theta}=25.5 \mathrm{~kg} \mathrm{~m}^{-3}-\mathrm{July} \quad 88$

Fig. 76: Pressure on $\sigma_{\theta}=26.0 \mathrm{~kg} \mathrm{~m}^{-3}-\mathrm{July} \quad 89$

Fig. 77: Temperature on $\sigma_{\theta}=26.0 \mathrm{~kg} \mathrm{~m}^{-3}-\mathrm{July} \quad 90$

Fig. 78: Salinity on $\sigma_{\theta}=26.0 \mathrm{~kg} \mathrm{~m}^{-3}-\mathrm{July} \quad 91$

Fig. 79: Pressure on $\sigma_{\theta}=26.5 \mathrm{~kg} \mathrm{~m}^{-3}-\mathrm{July} \quad 92$

Fig. 80: Temperature on $\sigma_{\theta}=26.5 \mathrm{~kg} \mathrm{~m}^{-3}-\mathrm{July} \quad 93$

Fig. 81: Salinity on $\sigma_{\theta}=26.5 \mathrm{~kg} \mathrm{~m}-3-J u l y \quad 94$

Fig. 82: Pressure on $\sigma_{\theta}=27.0 \mathrm{~kg} \mathrm{~m}^{-3}-\mathrm{July} \quad 95$

Fig. 83: Temperature on $\sigma_{\theta}=27.0 \mathrm{~kg} \mathrm{~m}^{-3}-\mathrm{July} \quad 96$

Fig. 84: Salfinity on $\sigma_{\theta}=27.0 \mathrm{~kg} \mathrm{~m}-3$ - July 97

Fig. 85: Pressure on $\sigma_{\theta}=25.0 \mathrm{~kg} \mathrm{~m}^{-3}$ - August 98

Fig. 86: Temperature on $\sigma_{\theta}=25.0 \mathrm{~kg} \mathrm{~m}$ - August 99

Fig. 87: Salinity on $\sigma_{\theta}=25.0 \mathrm{~kg} \mathrm{~m}-3$ - August 100

Fig. 88: Pressure on $\sigma_{\theta}=25.5 \mathrm{~kg} \mathrm{~m}^{-3}$ - August 101

Fig. 89: Temperature on $\sigma_{\theta}=25.5 \mathrm{~kg} \mathrm{~m}$ - August 102

Fig. 90: Salinity on $\sigma_{\theta}=25.5 \mathrm{~kg} \mathrm{~m}^{-3}$ - August 103

Fig. 91: Pressure on $\sigma_{\theta}=26.0 \mathrm{~kg} \mathrm{~m}-3$-August 104

Fig. 92: Temperature on $\sigma_{\theta}=26.0 \mathrm{~kg} \mathrm{~m}$ - August 105

Fig. 93: Salinity on $\sigma_{\theta}=26.0 \mathrm{~kg} \mathrm{~m}^{-3}$ - August 106 


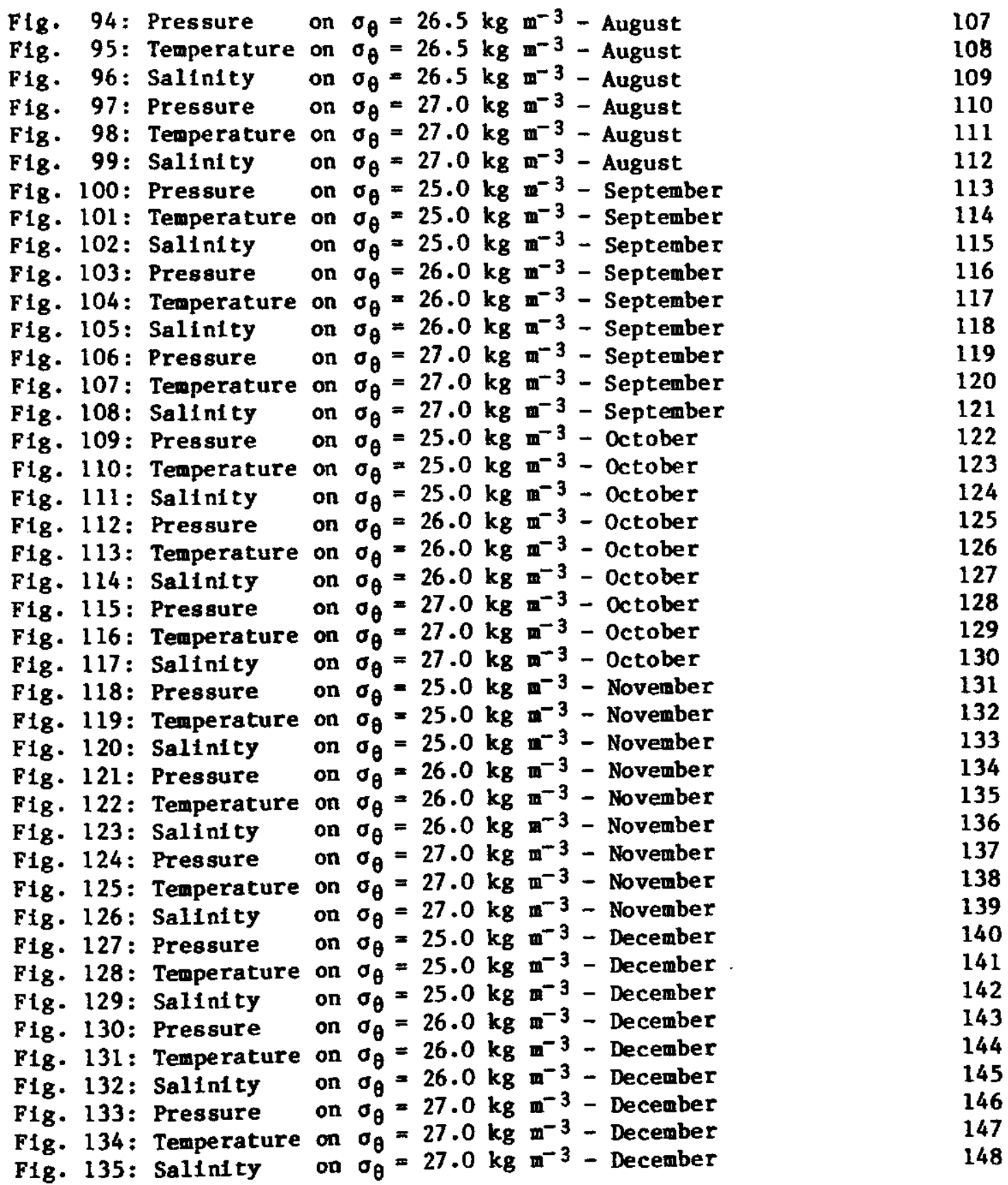


Monthly mean sectlons

Fig. 136: Location of vertical sections shown in figures 137-160 149

Fig. 137: Azores-Greenland - January

F1g. 138: Azores-Greenland - February

F1g. 139: Azores-Greenland - March

F1g. 140: Azores-Greenland - Apri1

F1g. 141: Azores-Greenland - May

Fig. 142: Azores-Greenland - June

Fig. 143: Azores-Greenland - July

Fig. 144: Azores-Greenland - August

Fig. 145: Azores-Greenland - September

F1g. 146: Azores-Greenland - October

F1g. 147: Azores-Greenland - November

Fig. 148: Azores-Greenland - December

Fig. 149: Azores-English Channel - January 162

Fig. 150: Azores-English Channel - February 163

Fig. 151: Azores-English Channel - March 164

F1g. 152: Azores-Eng1ish Channel - April 165

F1g. 153: Azores-English Channel - May 166

Fig. 154: Azores-English Channel - June 167

F1g. 155: Azores-English Channel - July 168

F1g. 156: Azores-English Channel - August 169

Fig. 157: Azores-Eng11sh Channel - September 170

F1g. 158: Azores-Eng11sh Channel - October 171

Fig. 159: Azores-Eng11sh Channel - November 172

F1g. 160: Azores-Eng1ish Channel - Deceaber 173 


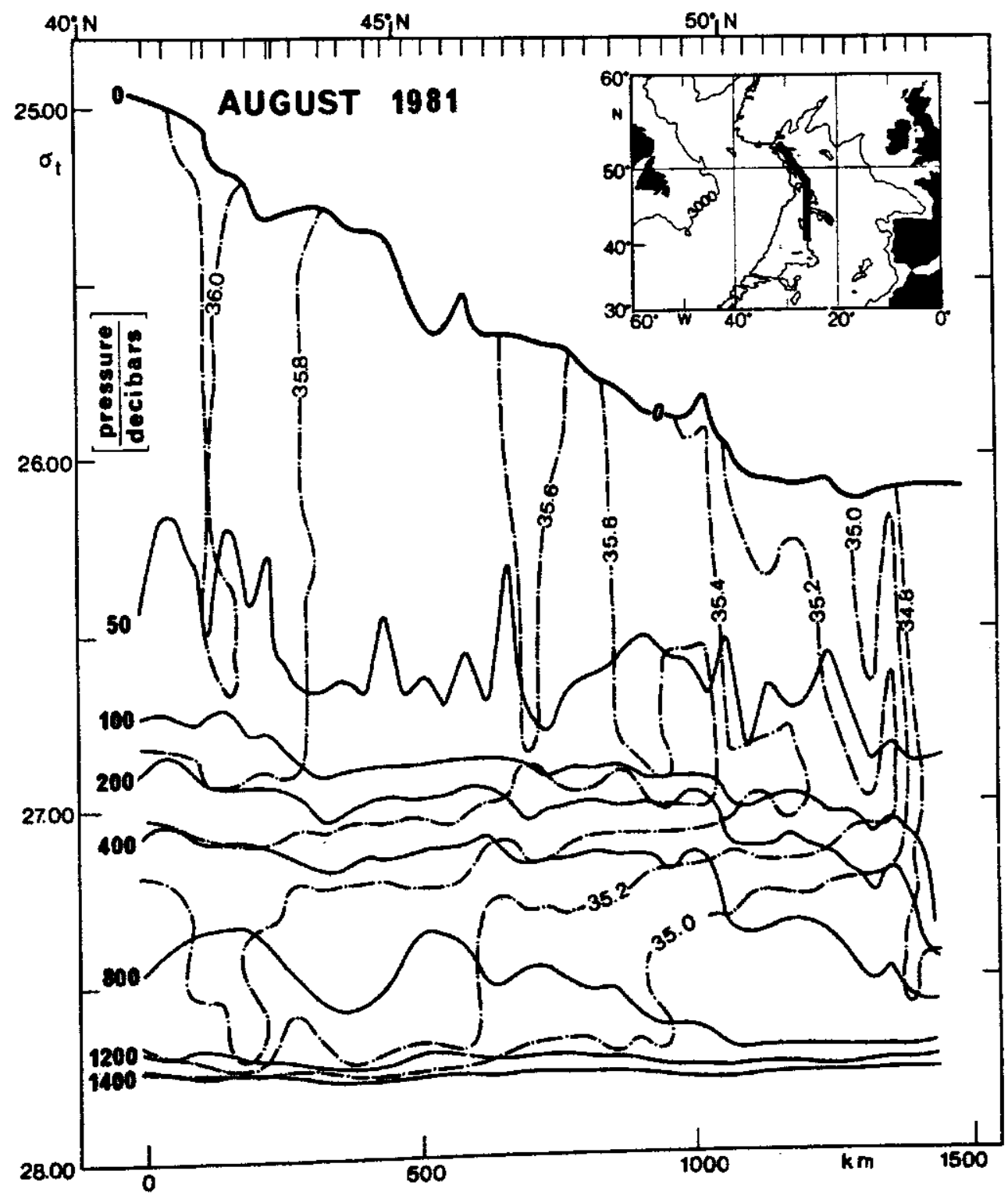

Fig. 1: Isopycnic presentation of the distribution of salinity and pressure in hydrographic section going north from the Arores (data from Meincke \& Sy, 1983). 


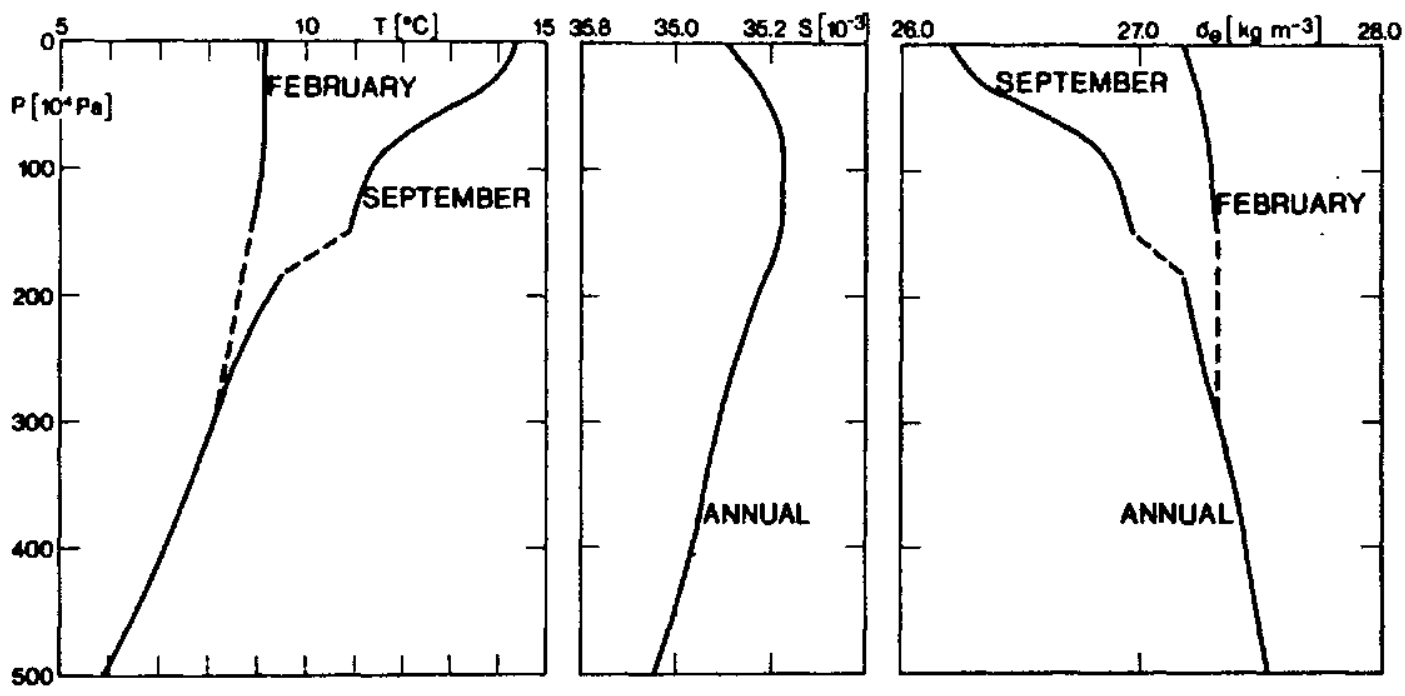

Fig. 2: Monthly mean profiles of temperature, salinity and density from the atlas for the $1^{\circ} \times 1^{\circ}$ square at position $50^{\circ} \mathrm{N} 35^{\circ} \mathrm{W}$ in February and September. The broken lines indicate the procedure used to obtain monthly mean values in the seasonally-varying layer below 150 metres.

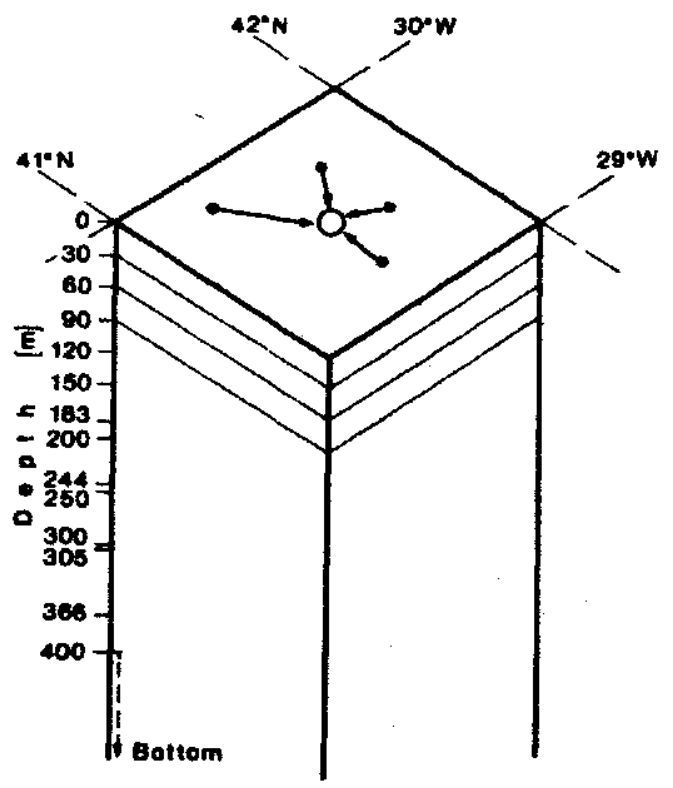

Fig. 3: Horizontal and vertical positloning of the atlas data. The data cycle containing the mean values calculated by overaging the samples lying between $41^{\circ} \mathrm{N}$ and $42^{\circ} \mathrm{N}$ and $29^{\circ} \mathrm{W}$ and $30^{\circ} \mathrm{W}$ are labeled at the position: $\phi=41^{\circ} 30^{\prime}, \lambda=-29^{\circ}-30^{\prime}$. 
Fig. 4:

PRESSURE $\left(10^{4} \mathrm{~Pa}\right)$ on $\sigma_{\theta}=25.0 \mathrm{~kg} \mathrm{~m}^{-3}$ JANUARY

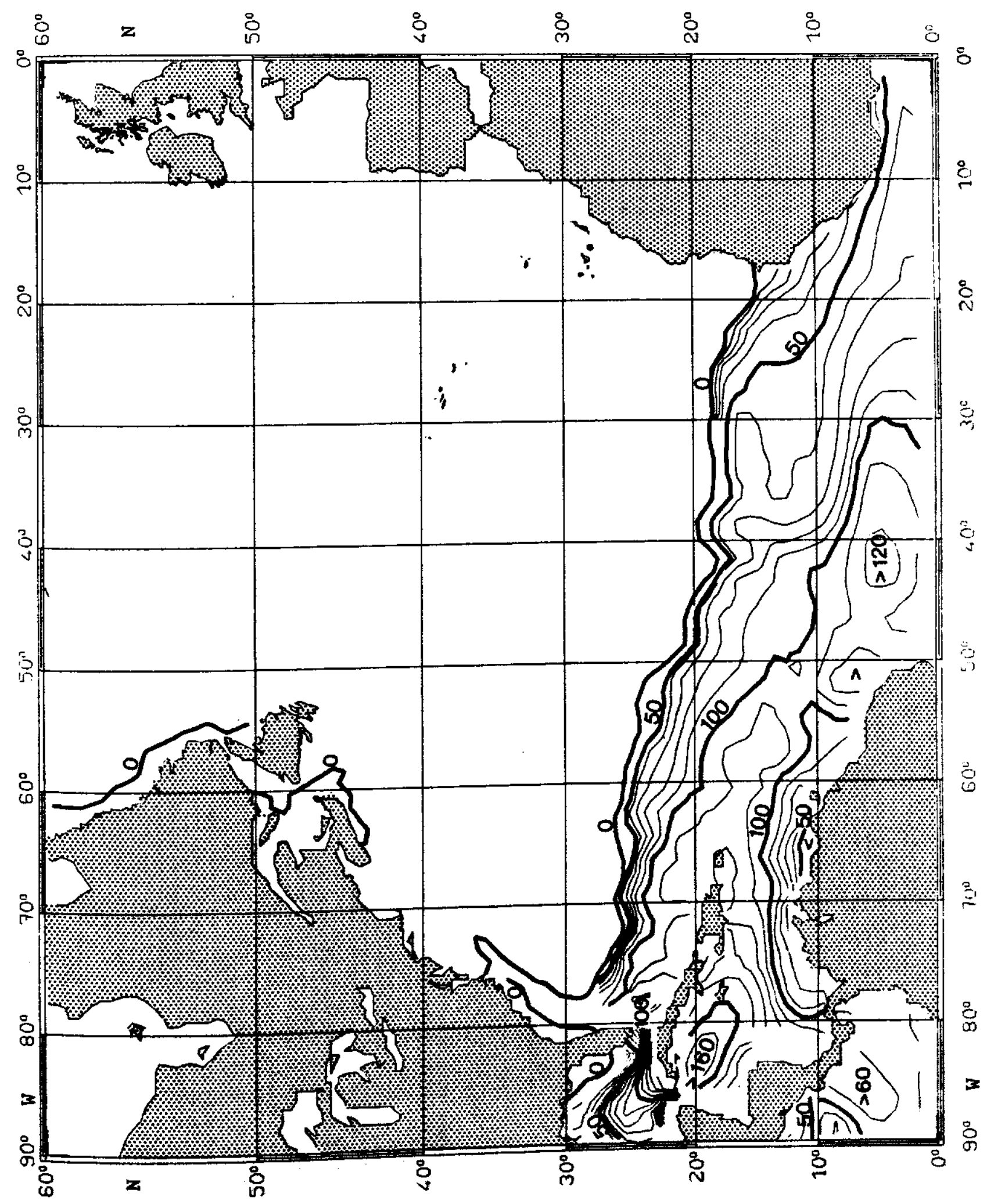


Fig. 5:

TEMPERATURE $\left({ }^{\circ} \mathrm{C}\right)$ on $\sigma_{\theta}=25.0 \mathrm{~kg} \mathrm{~m}^{-3}$ JANUARY

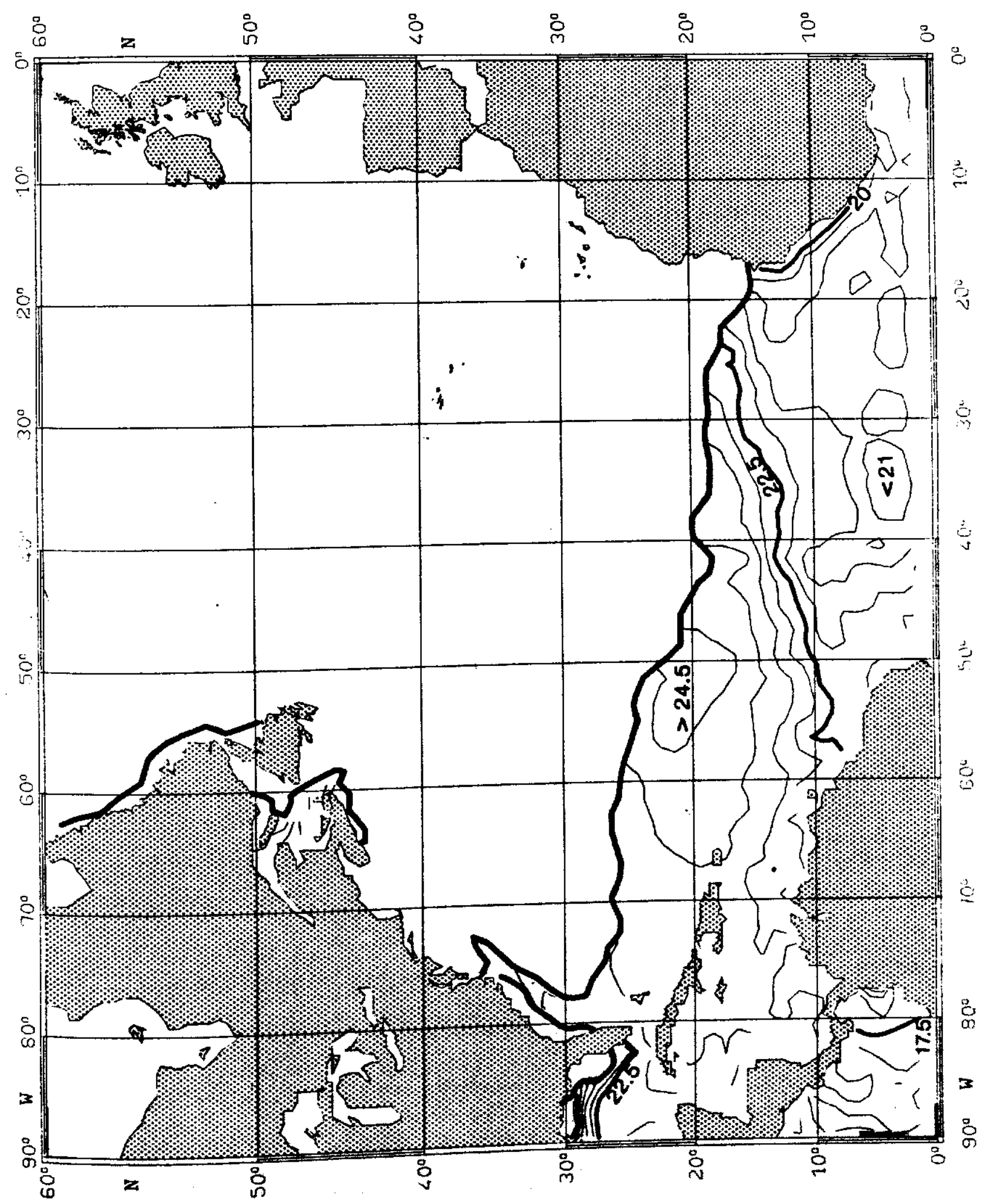


Fig. 6:

SALINITY $\left(10^{-3}\right)$ on $\sigma_{\theta}=25.0 \mathrm{~kg} \mathrm{~m}^{-3} \quad$ JANUARY

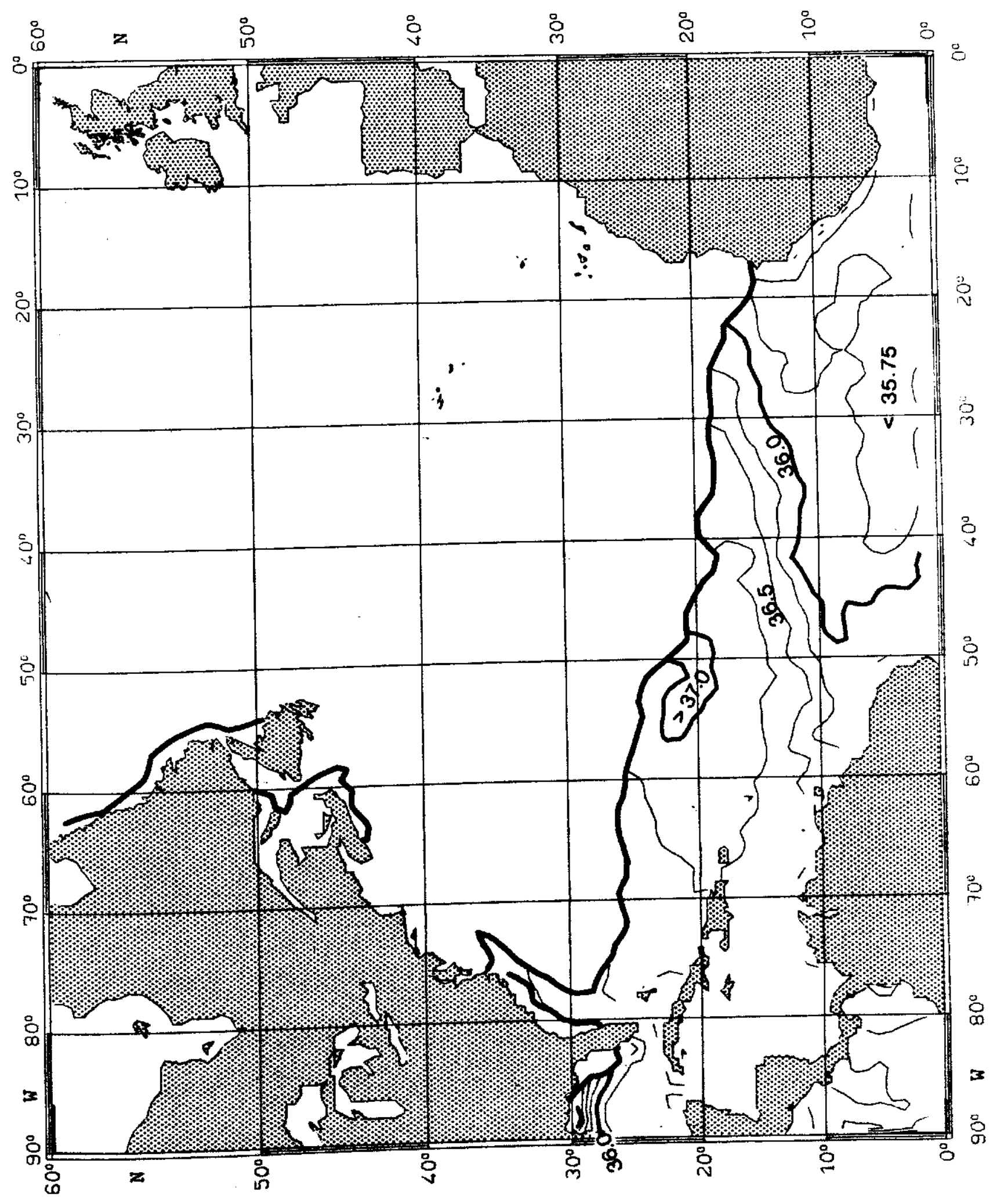


Fig. 7:

PRESSURE $\left(10^{4} \mathrm{~Pa}\right)$ on $\sigma_{\theta}=26.0 \mathrm{~kg} \mathrm{~m}^{-3}$ JANUARY

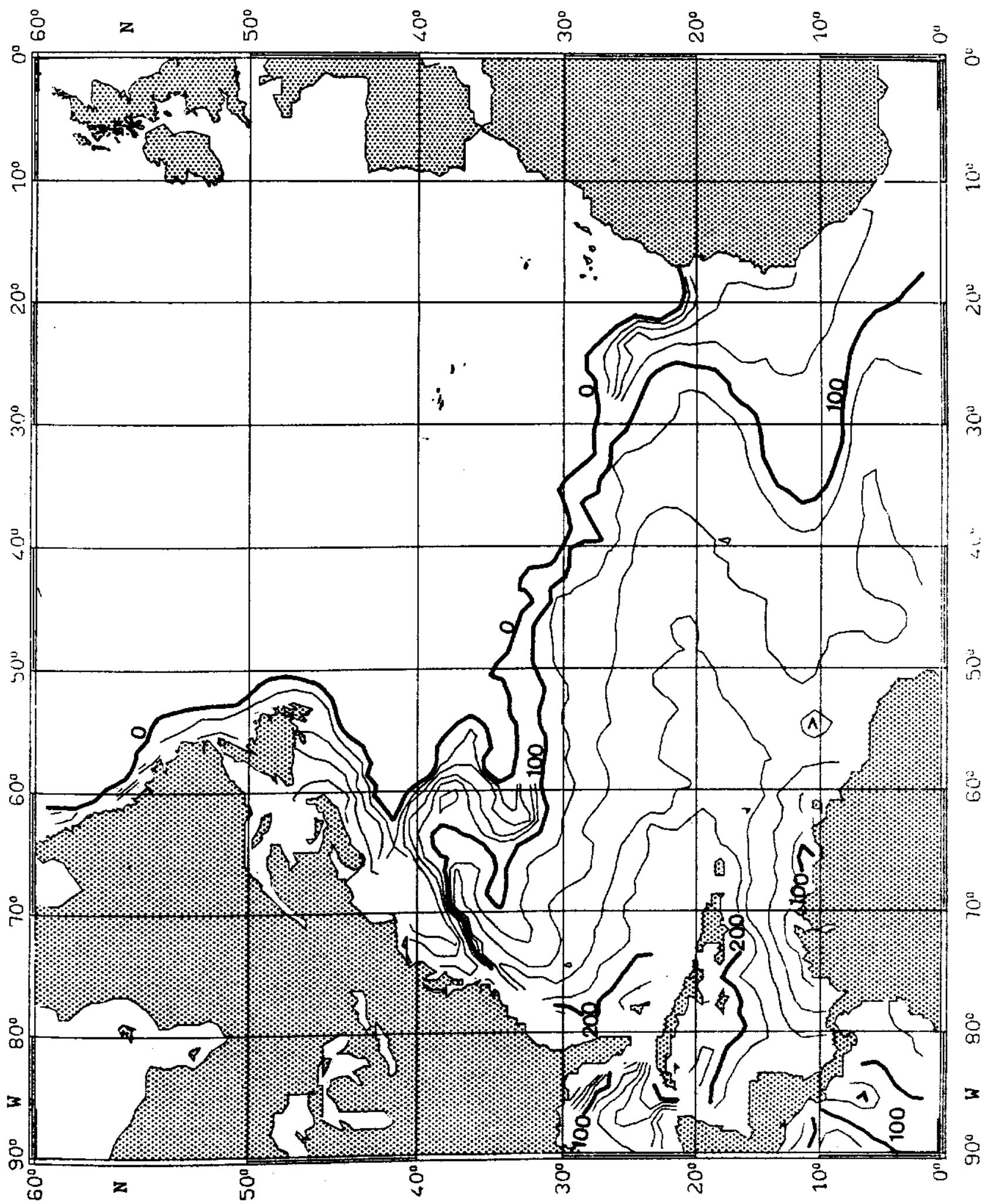


Fig. 8:

TEMPERATURE $\left({ }^{\circ} \mathrm{C}\right)$ on $\sigma_{\theta}=26.0 \mathrm{~kg} \mathrm{~m}^{-3}$ JANUARY

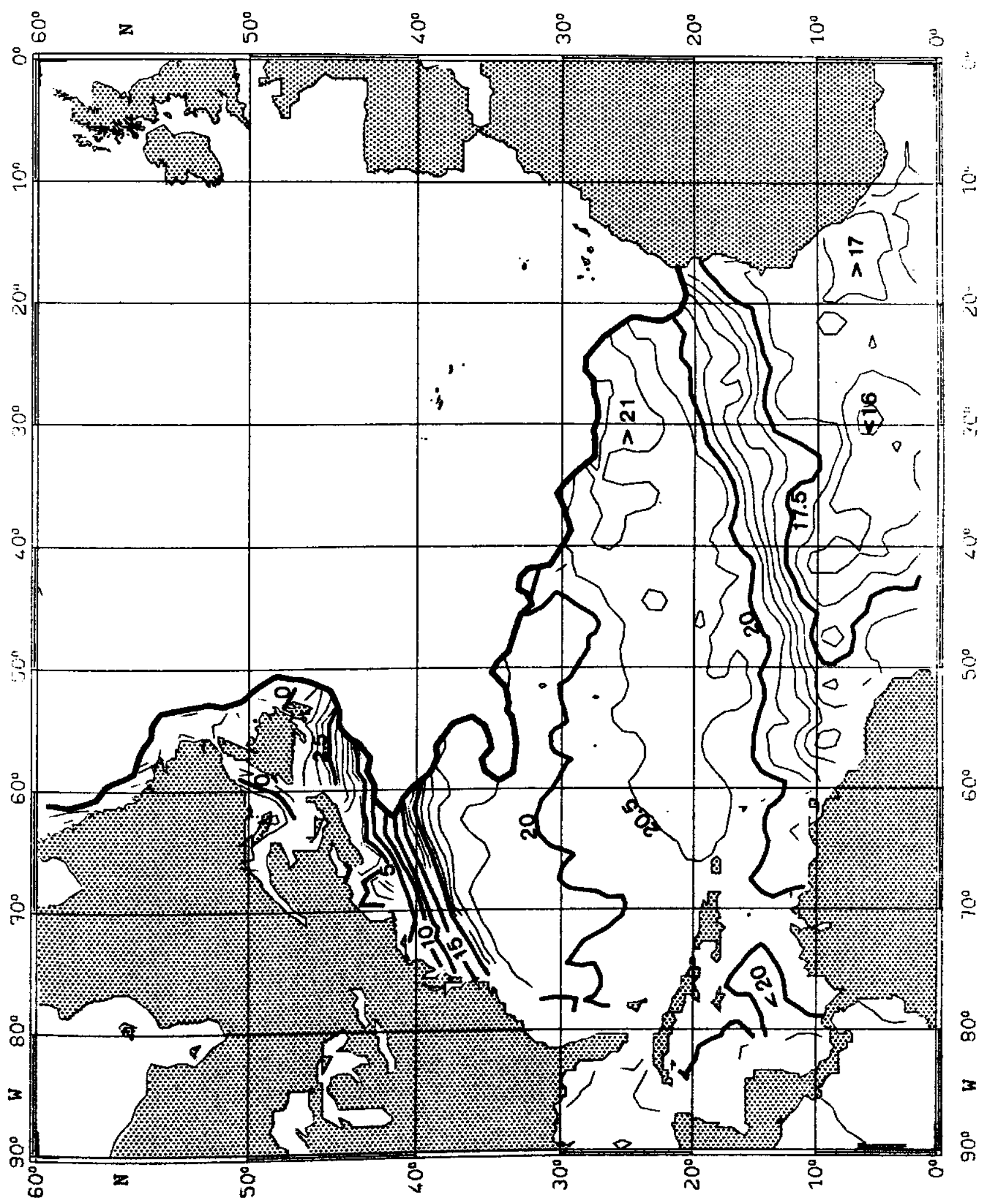


Fig. 9:

SALINITY $\left(10^{-3}\right)$ on $\sigma_{\theta}=26.0 \mathrm{~kg} \mathrm{~m}^{-3}$ JANUARY

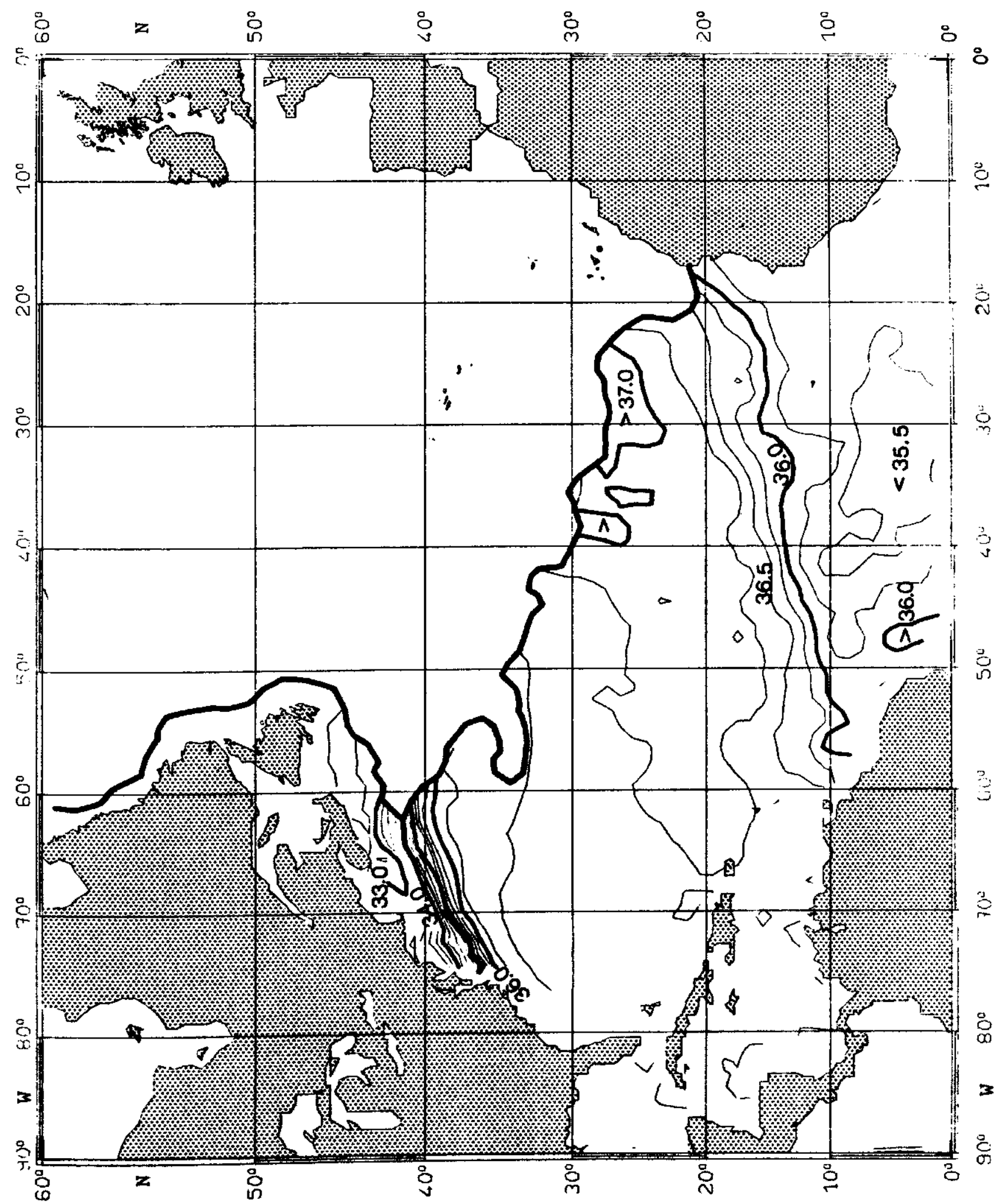


Fig. 10:

PRESSURE $\left(10^{4} \mathrm{~Pa}\right)$ on $\sigma_{\theta}=27.0 \mathrm{~kg} \mathrm{~m}^{-3}$ JANUARY

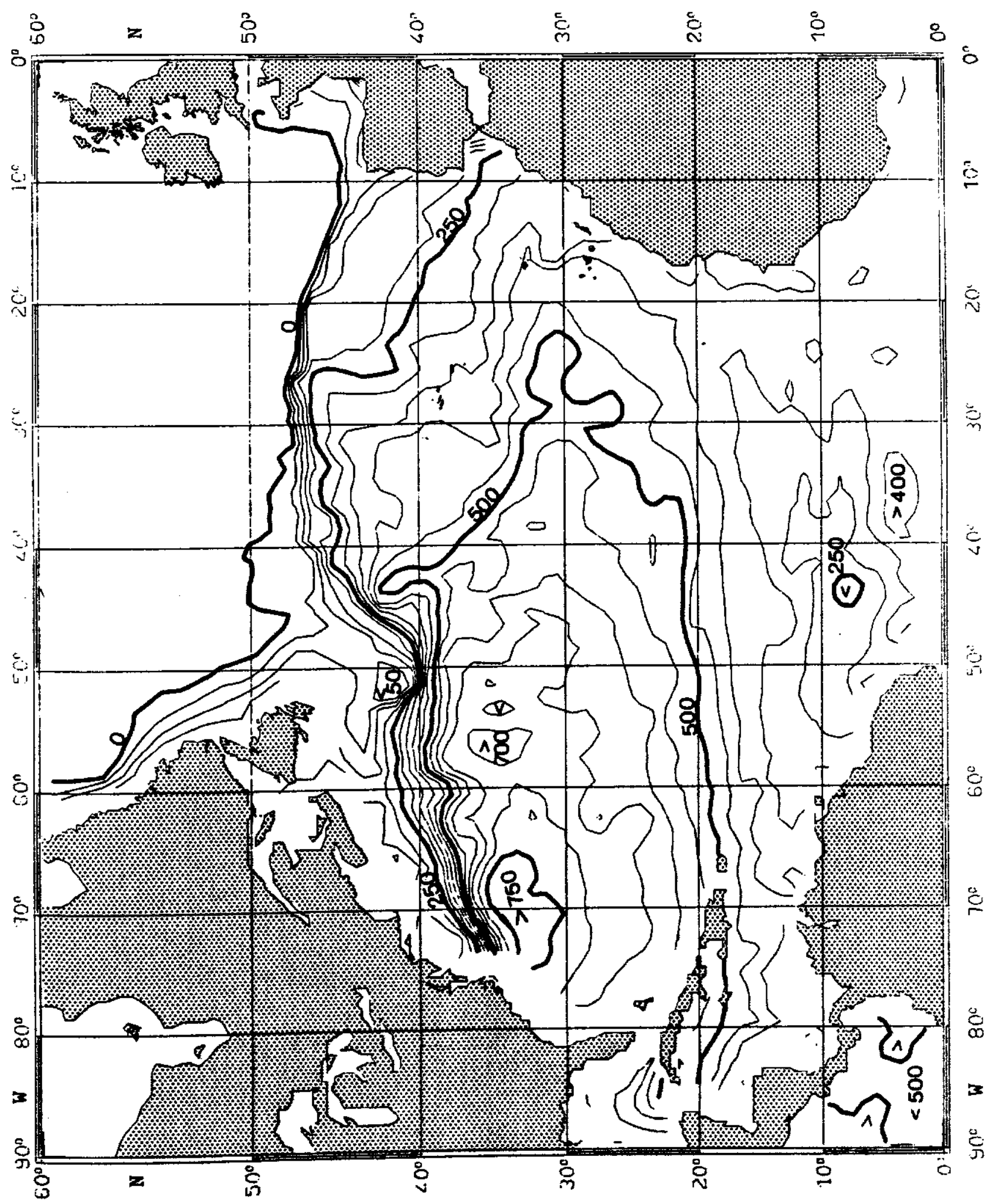


Fig. 11:

TEMPERATURE $\left({ }^{\circ} \mathrm{C}\right)$ on $\sigma_{\theta}=27.0 \mathrm{~kg} \mathrm{~m}^{-3}$ JANUARY

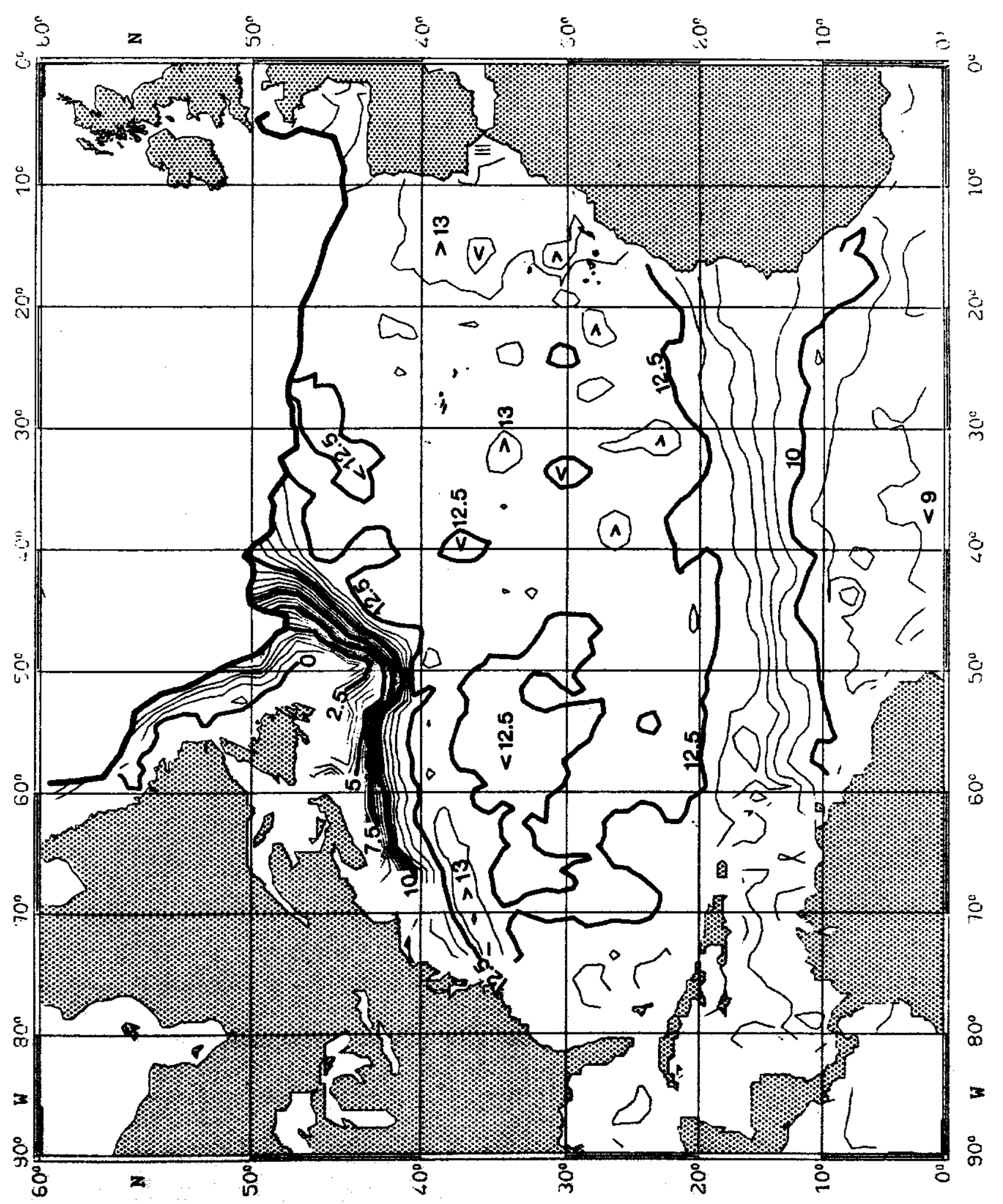


Fig. 12:

SALINITY $\left(10^{-3}\right)$ on $\sigma_{\theta}=27.0 \mathrm{~kg} \mathrm{~m}^{-3} \quad$ JANUARY

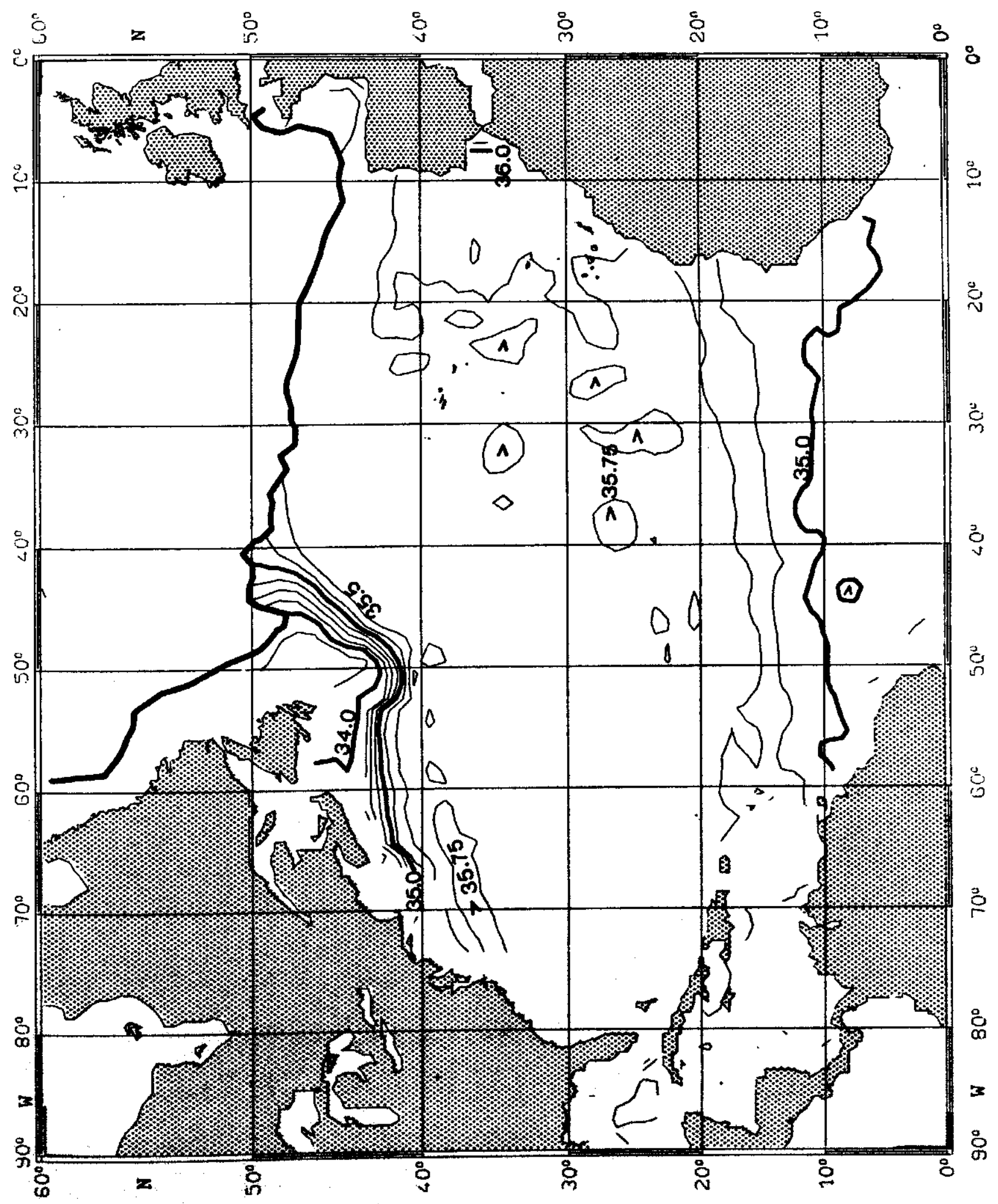


Fig. 13:

PRESSURE $\left(10^{4} \mathrm{~Pa}\right)$ on $\sigma_{\theta}=25.0 \mathrm{~kg}^{-3}$

FEBRUARY

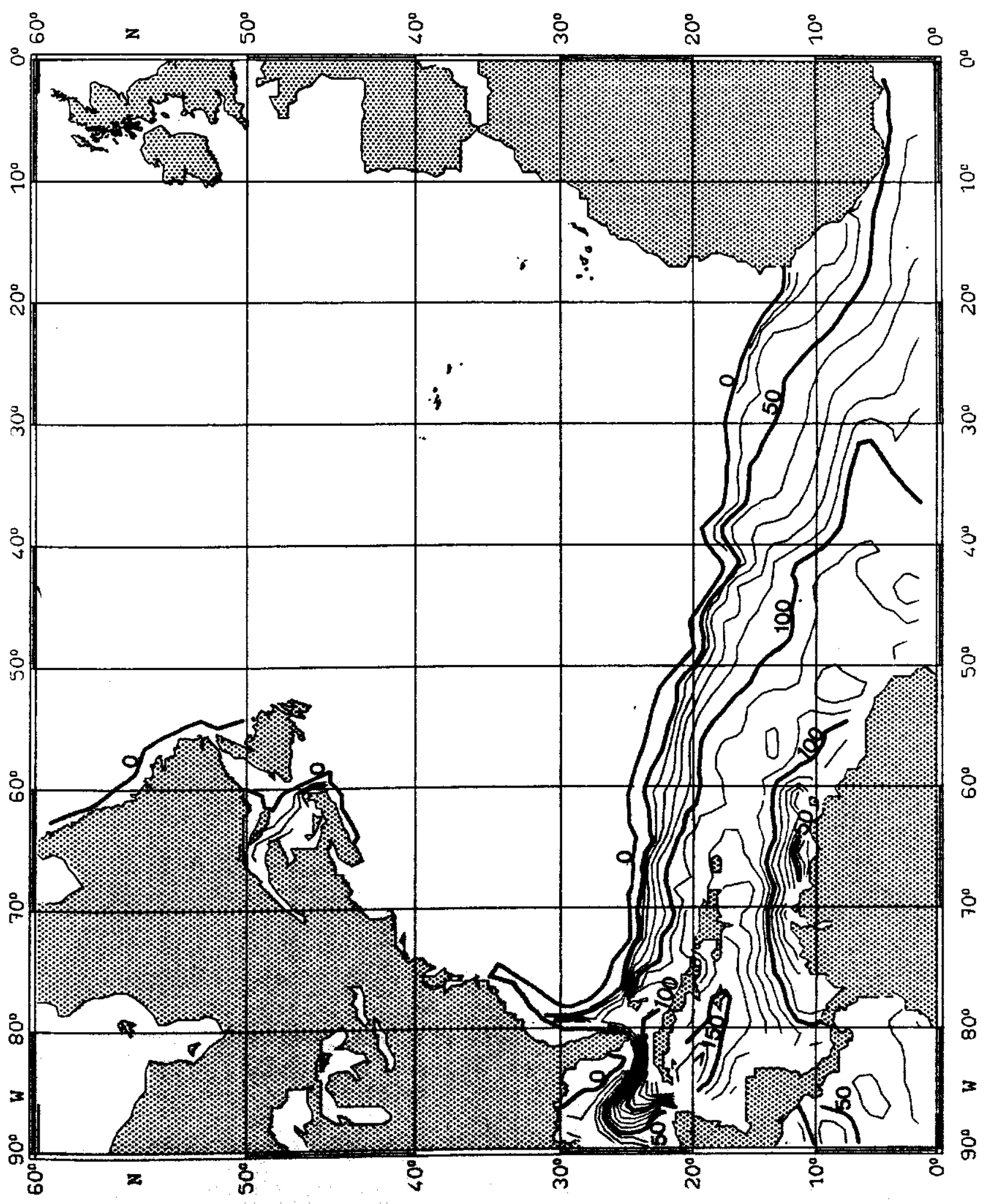


Fig. 14:

TEMPERATURE $\left({ }^{\circ} \mathrm{C}\right)$ on $\sigma_{\theta}=25.0 \mathrm{~kg} \mathrm{~m}^{-3} \quad$ FEBRUARY

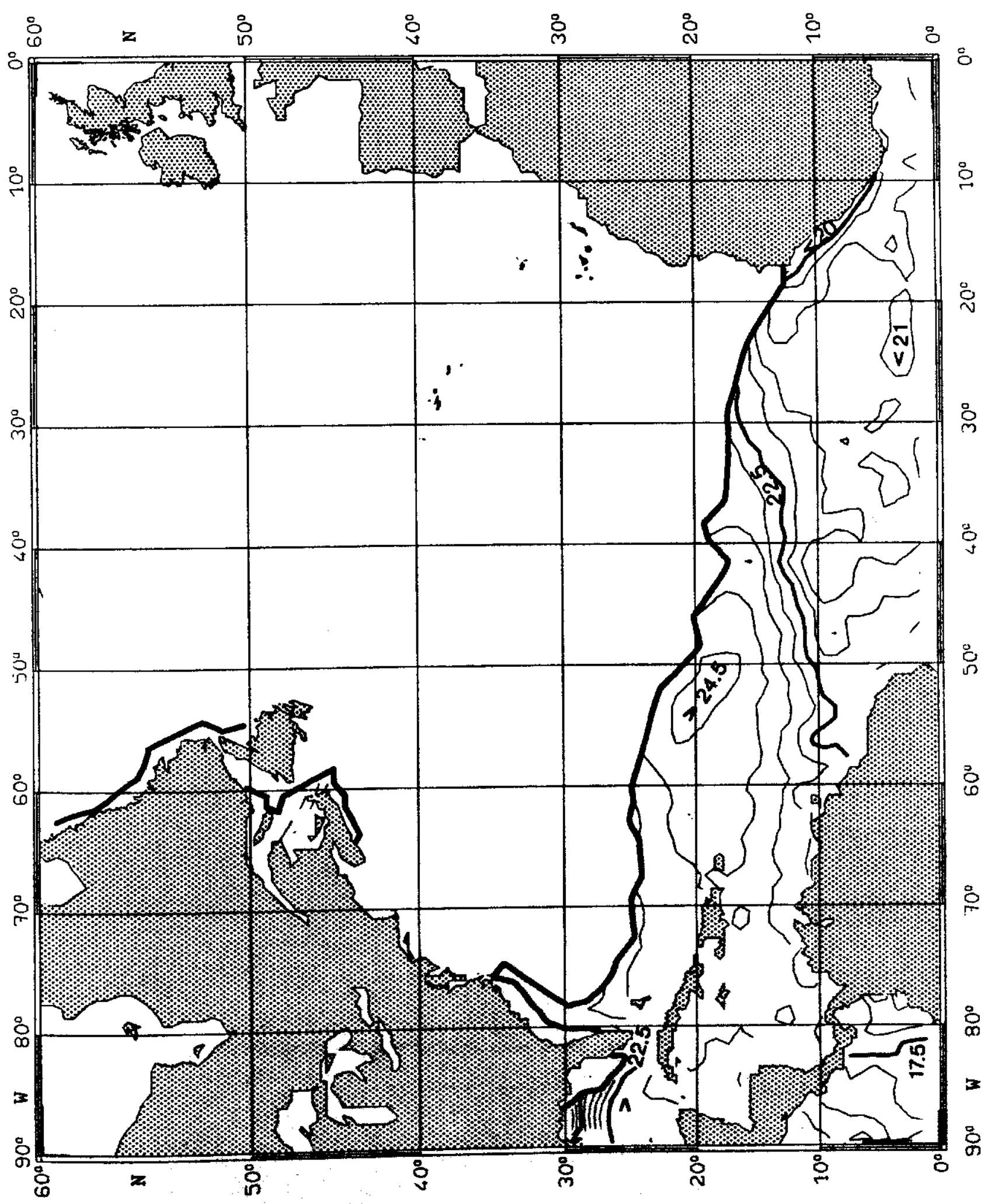


Fig. 15:

SALINITY $\left(10^{-3}\right)$ on $\sigma_{\theta}=25.0 \mathrm{~kg} \mathrm{~m}^{-3} \quad$ FEBRUARY

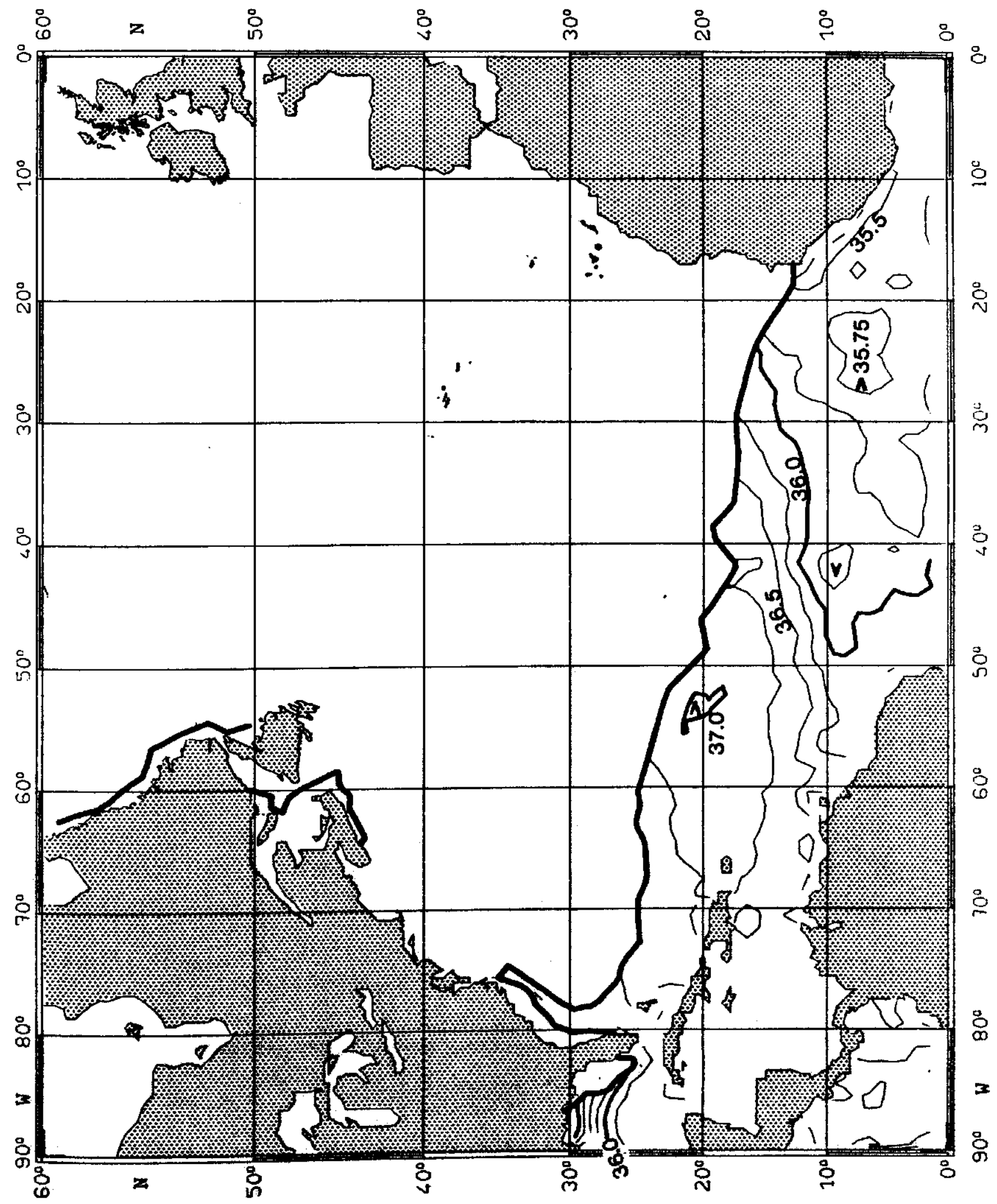


Fig. 16:

PRESSURE $\left(10^{4} \mathrm{~Pa}\right)$ on $\sigma_{\theta}=26.0 \mathrm{~kg} \mathrm{~m}^{-3} \quad$ FEBRUARY

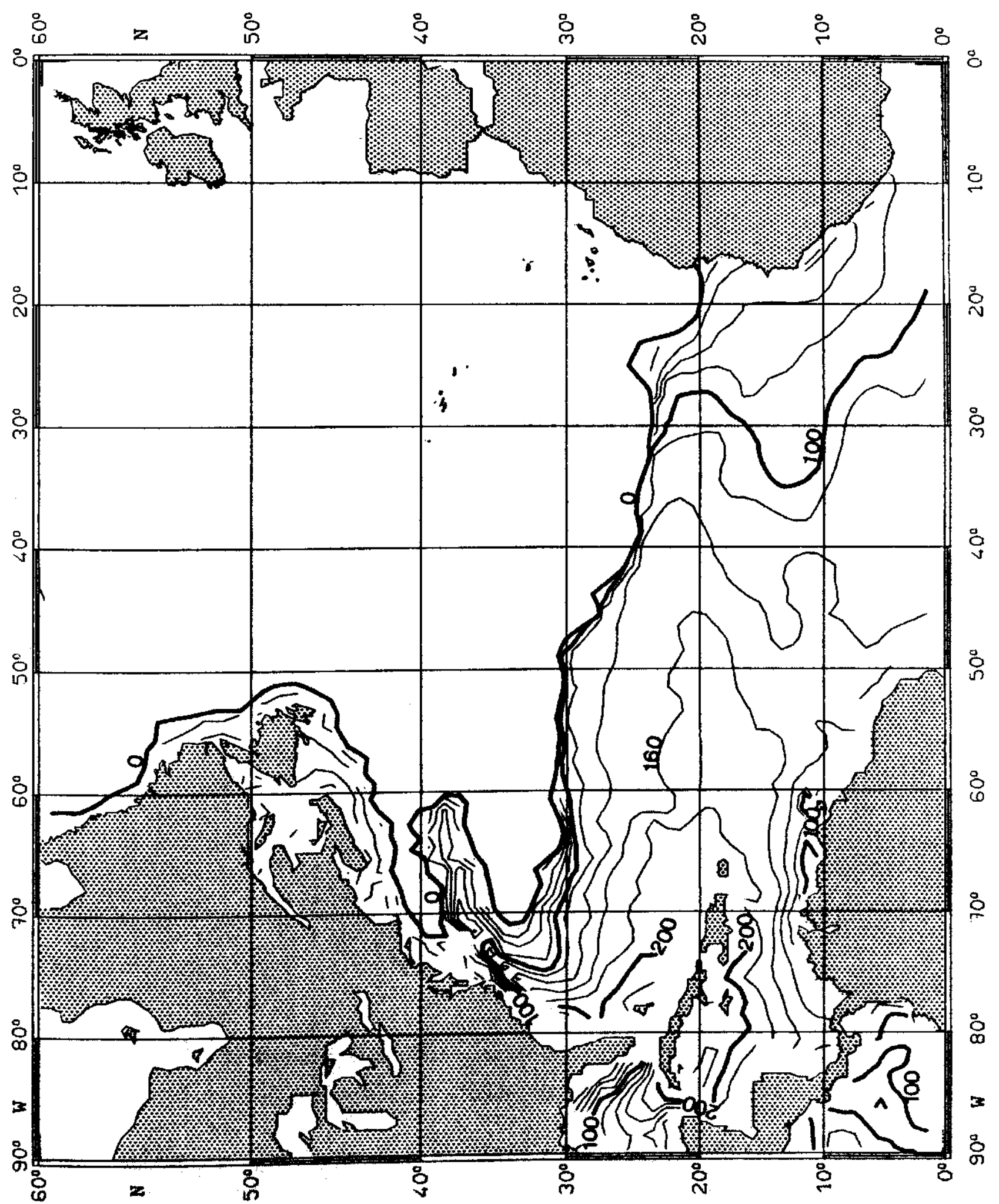


Fig. 17:

TEMPERATURE $\left({ }^{\circ} \mathrm{C}\right)$ on $\sigma_{\theta}=26.0 \mathrm{~kg} \mathrm{~m}$

FEBRUARY

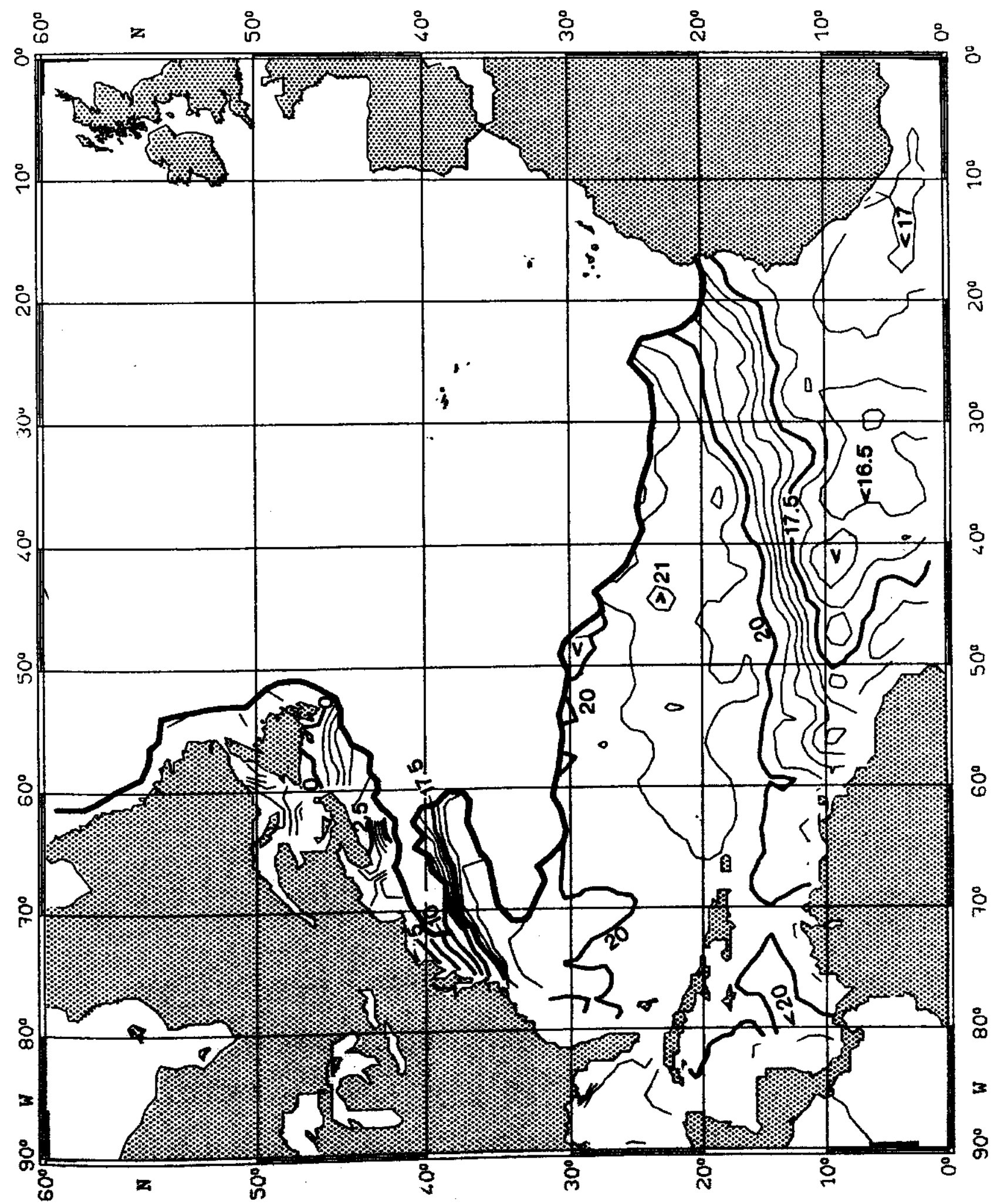


Fig. 18:

SALINITY $\left(10^{-3}\right)$ on $\sigma_{\theta}=26.0 \mathrm{~kg} \mathrm{~m}^{-3} \quad$ FEBRUARY

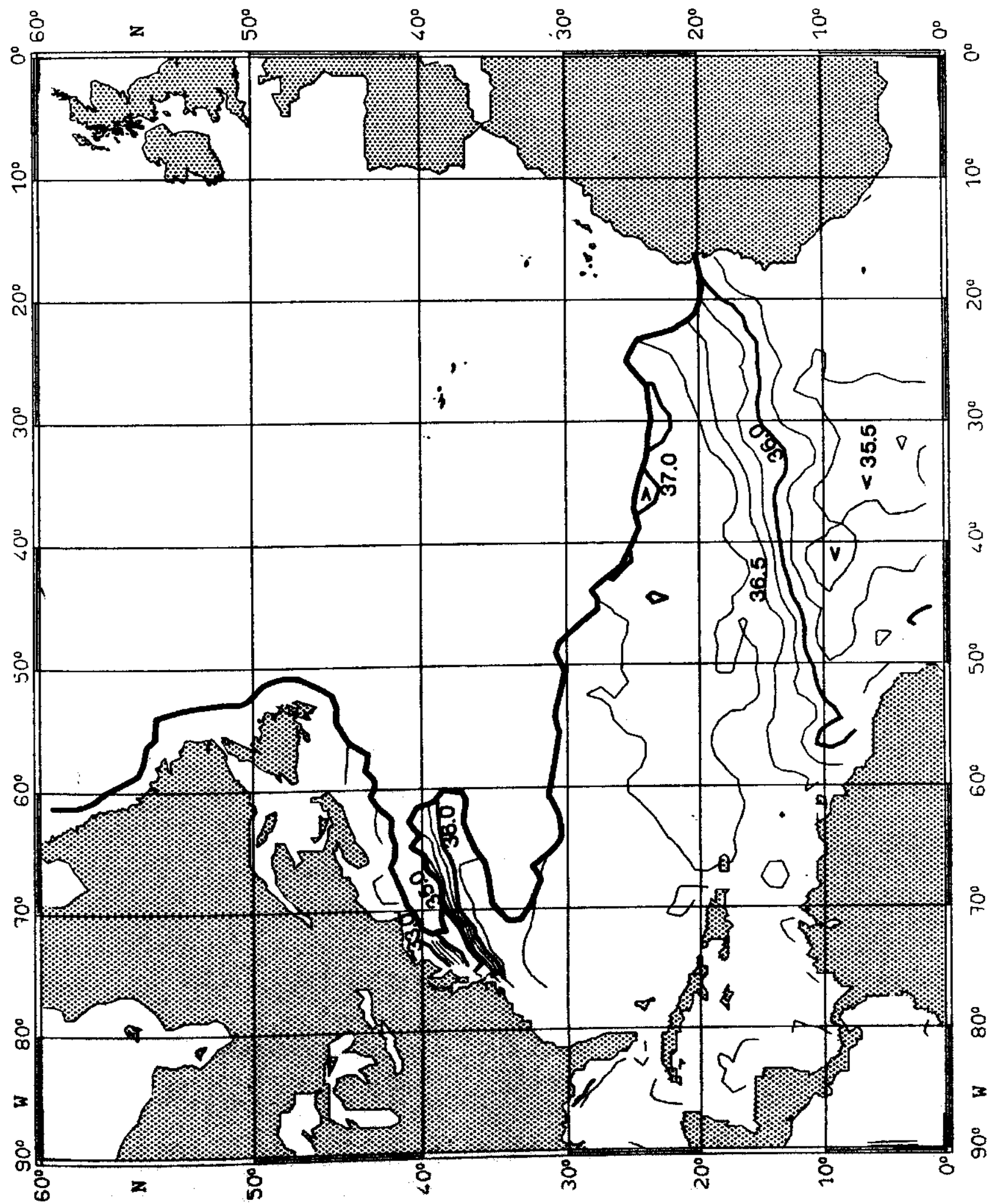


Fig. 19:

PRESSURE $\left(10^{4} \mathrm{~Pa}\right)$ on $\sigma_{\theta}=27.0 \mathrm{~kg} \mathrm{~m}^{-3} \quad$ FEBRUARY

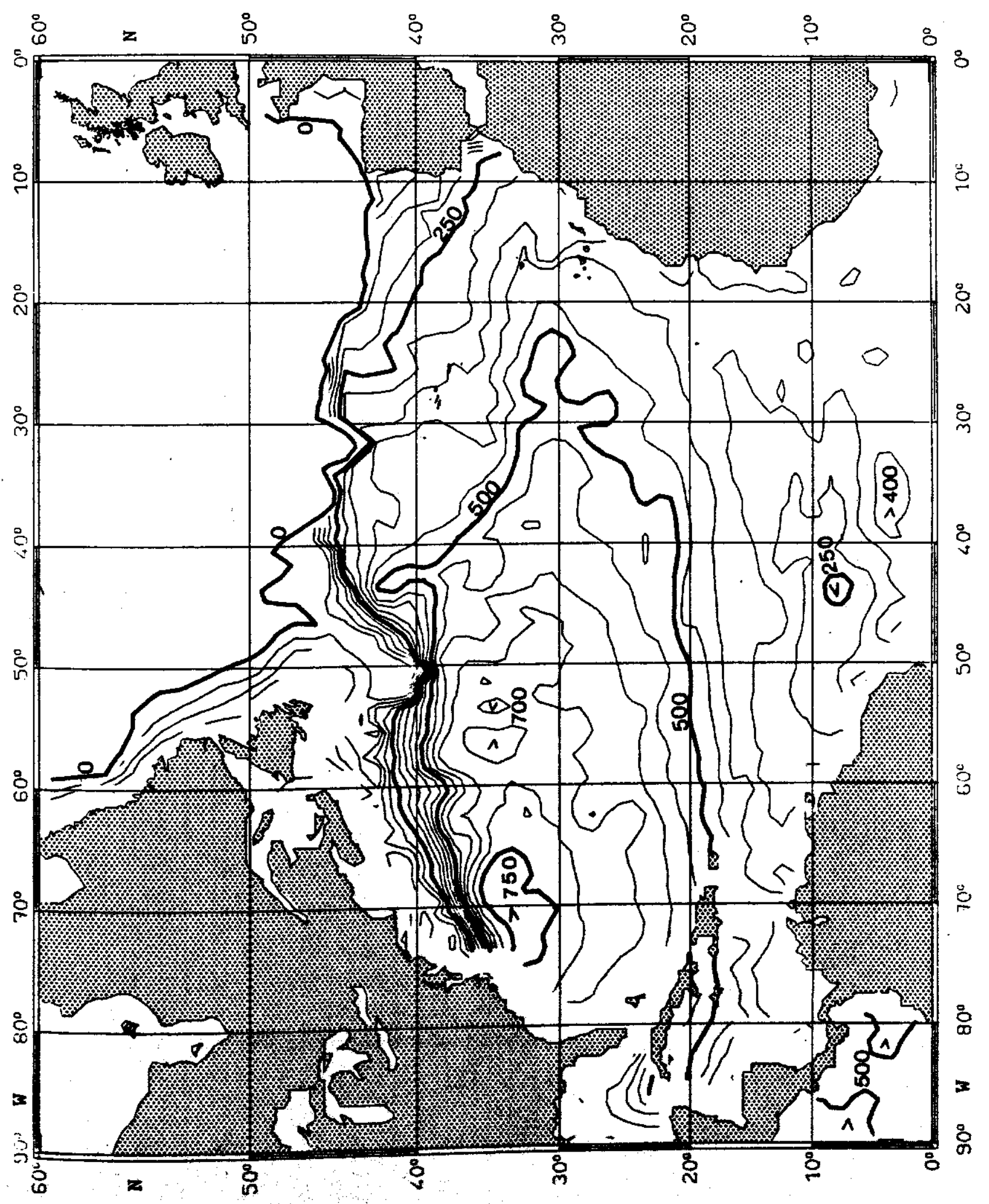


Fig. 20:

TEMPERATURE $\left({ }^{\circ} \mathrm{C}\right)$ on $\sigma_{\theta}=27.0 \mathrm{~kg} \mathrm{~m}^{-3}$ FEBRUARY

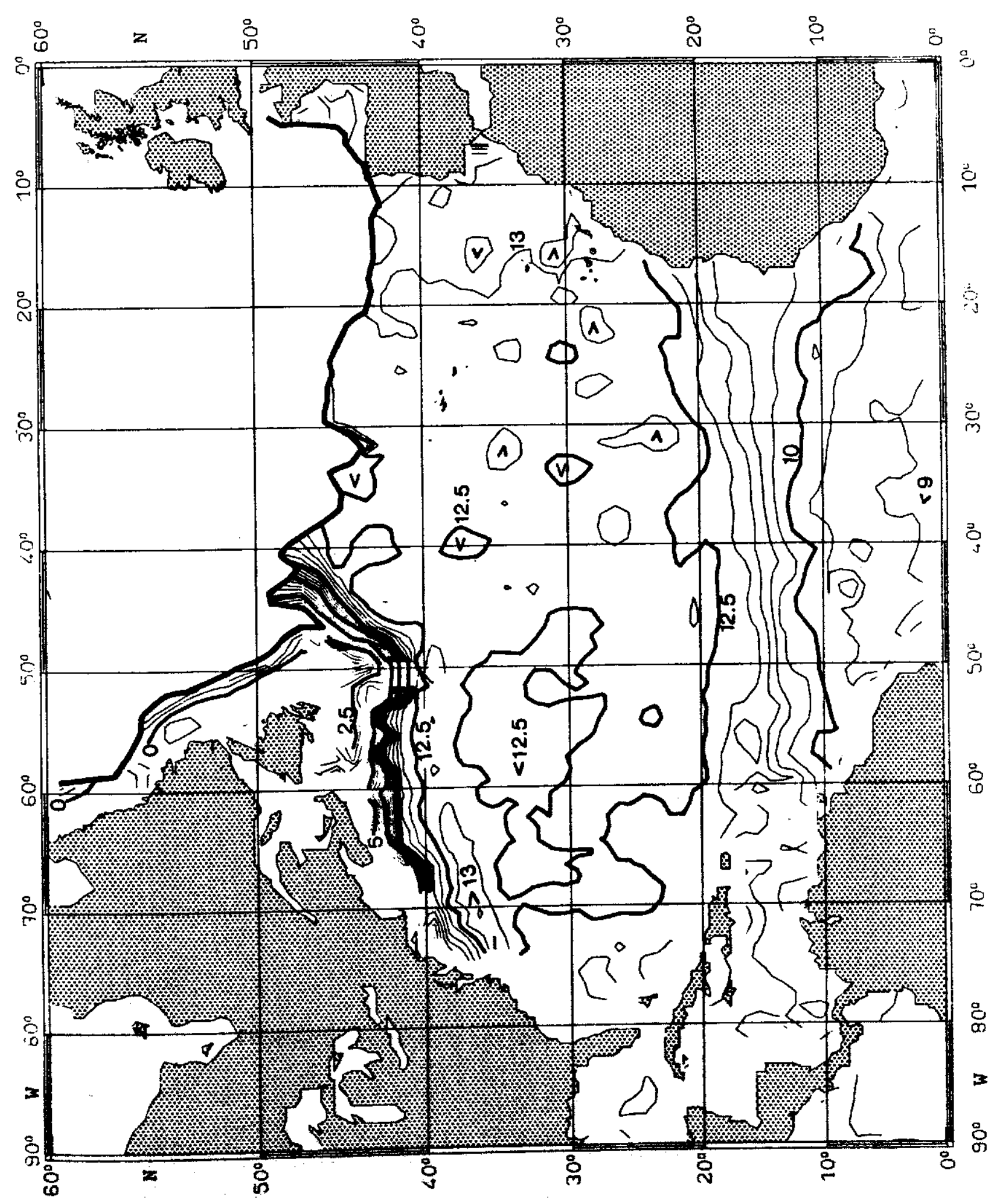


Fig. 21:

SALINITY $\left(10^{-3}\right)$ on $\sigma_{\theta}=27.0 \mathrm{~kg} \mathrm{~m}^{-3} \quad$ FEBRUARY

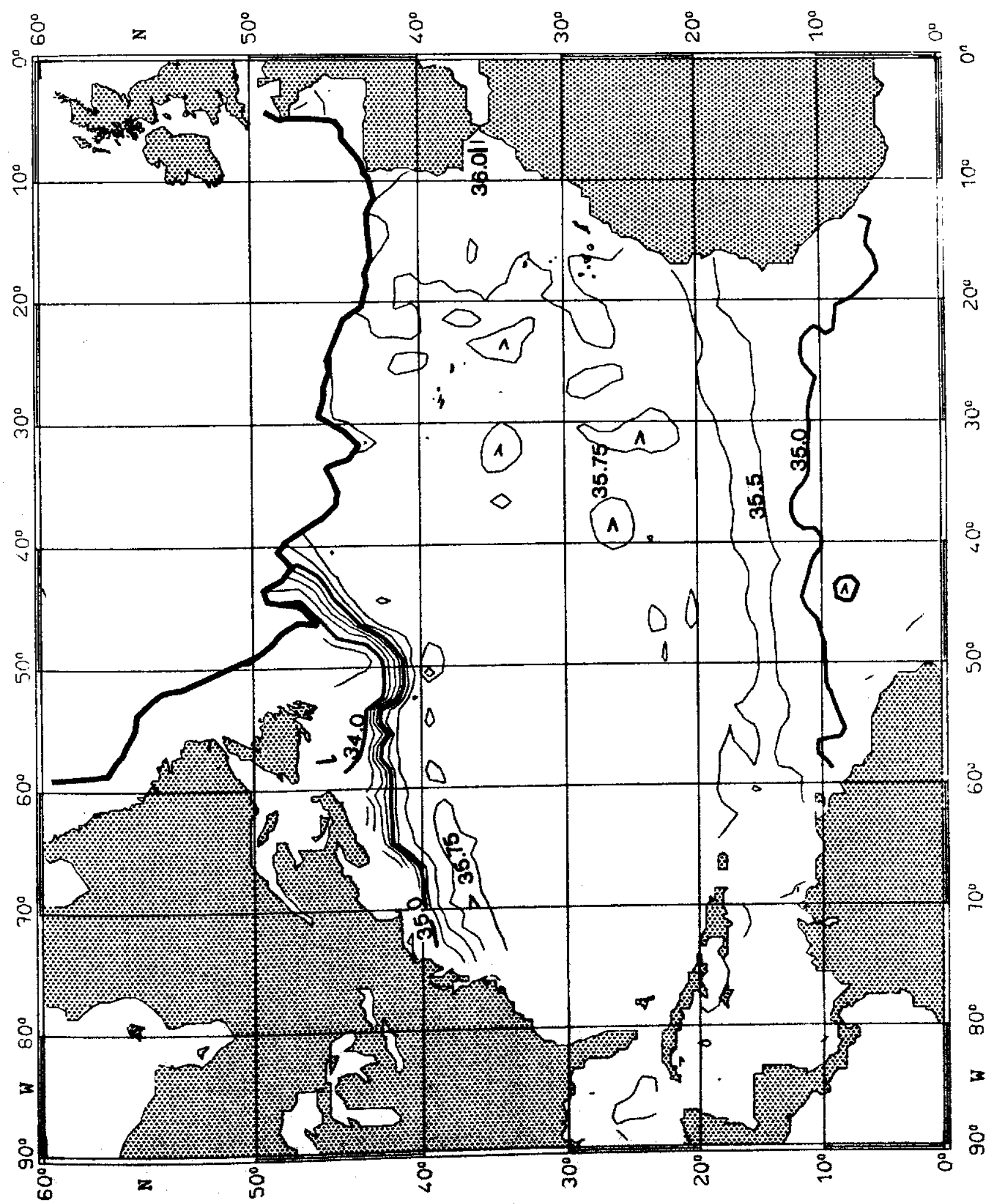


Fig. 22:

PRESSURE $\left(10^{4} \mathrm{~Pa}\right)$ on $\sigma_{\theta}=27.5 \mathrm{~kg} \mathrm{~m}^{-3} \quad$ FEBRUARY

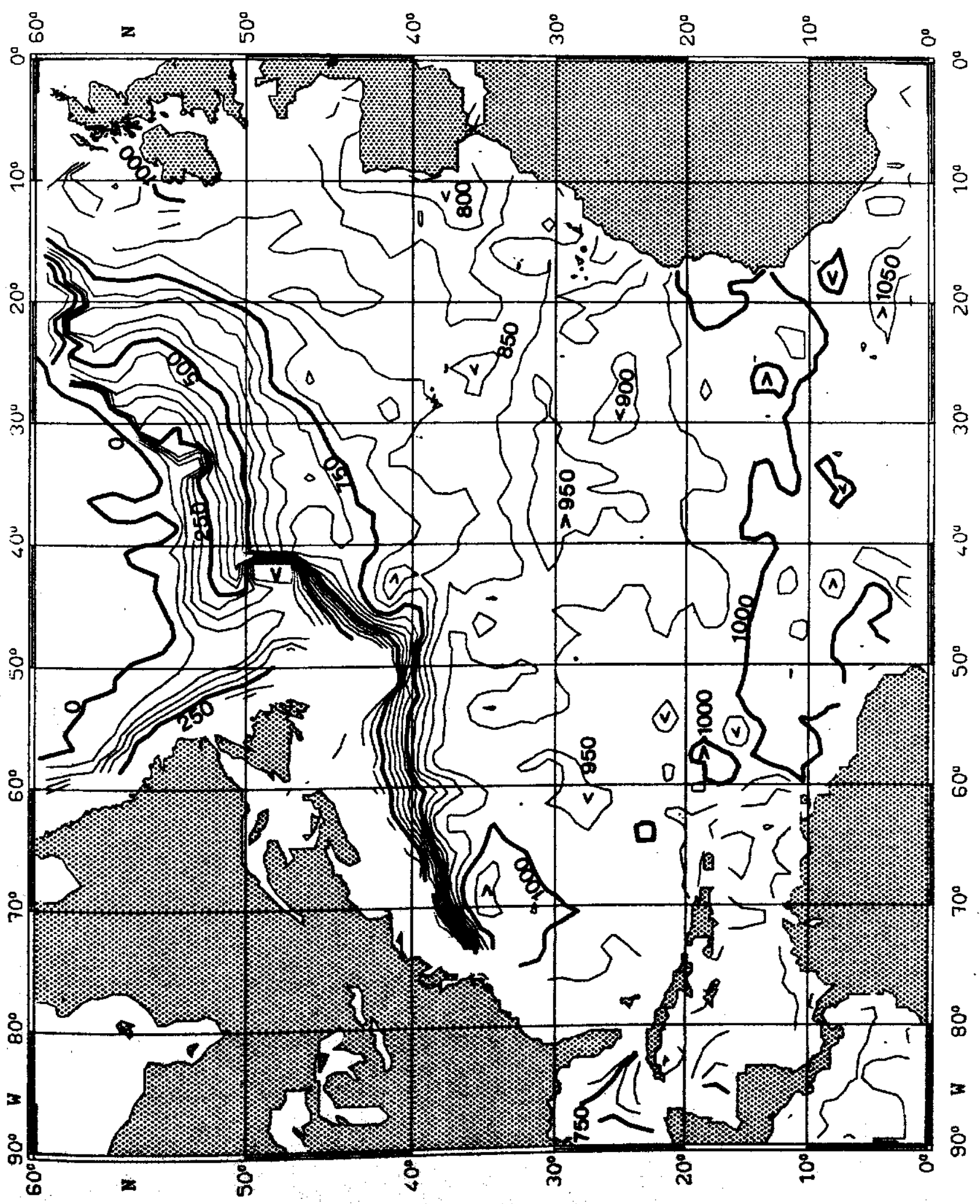


Fig. 23:

TEMPERATURE $\left({ }^{\circ} \mathrm{C}\right)$ on $\sigma_{\theta}=27.5 \mathrm{~kg} \mathrm{~m}^{-3} \quad$ FEBRUARY

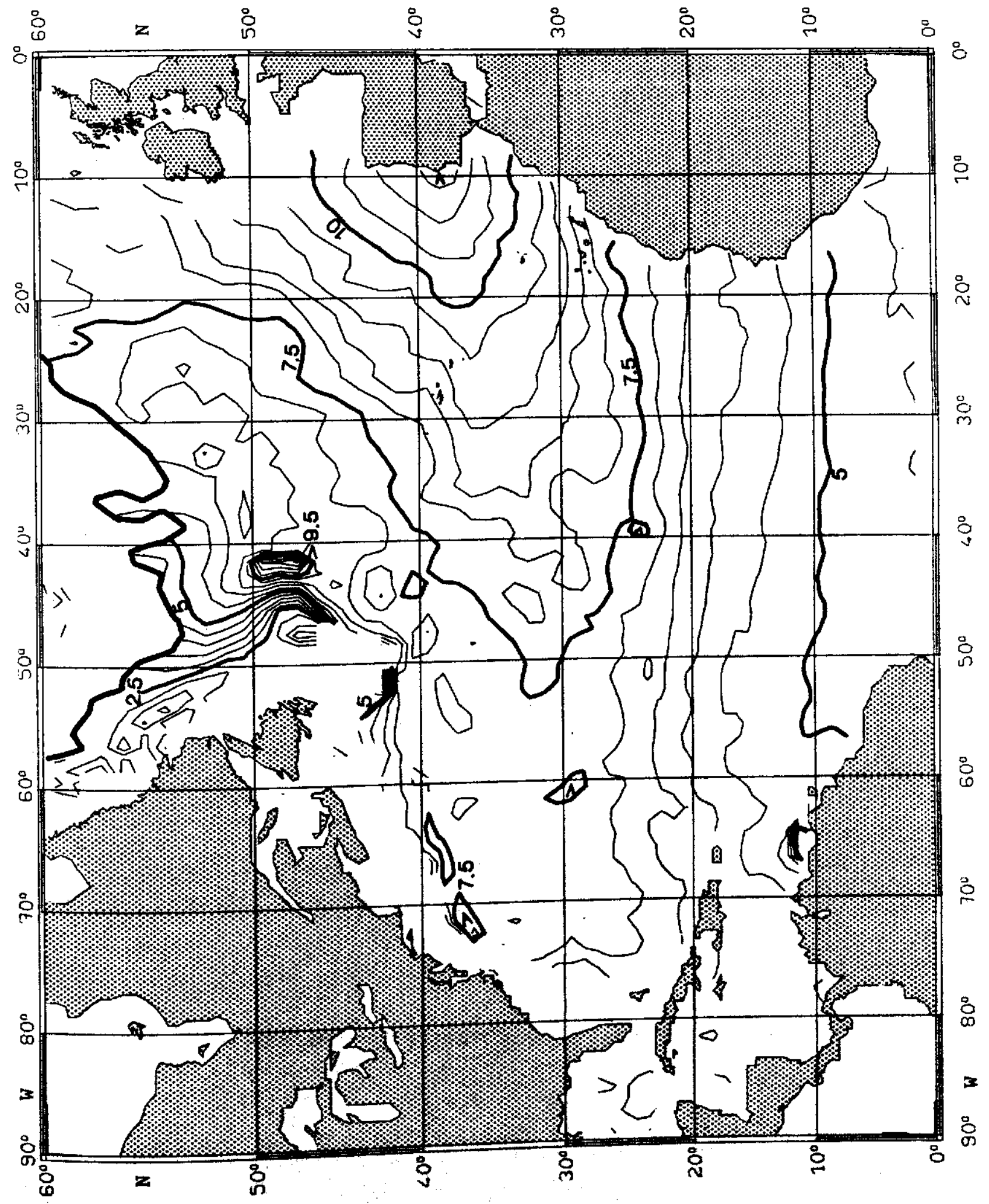


Fig. 24:

$\operatorname{SALINITY}\left(10^{-3}\right)$ on $\sigma_{\theta}=27.5 \mathrm{~kg} \mathrm{~m}^{-3}$

FEBRUARY

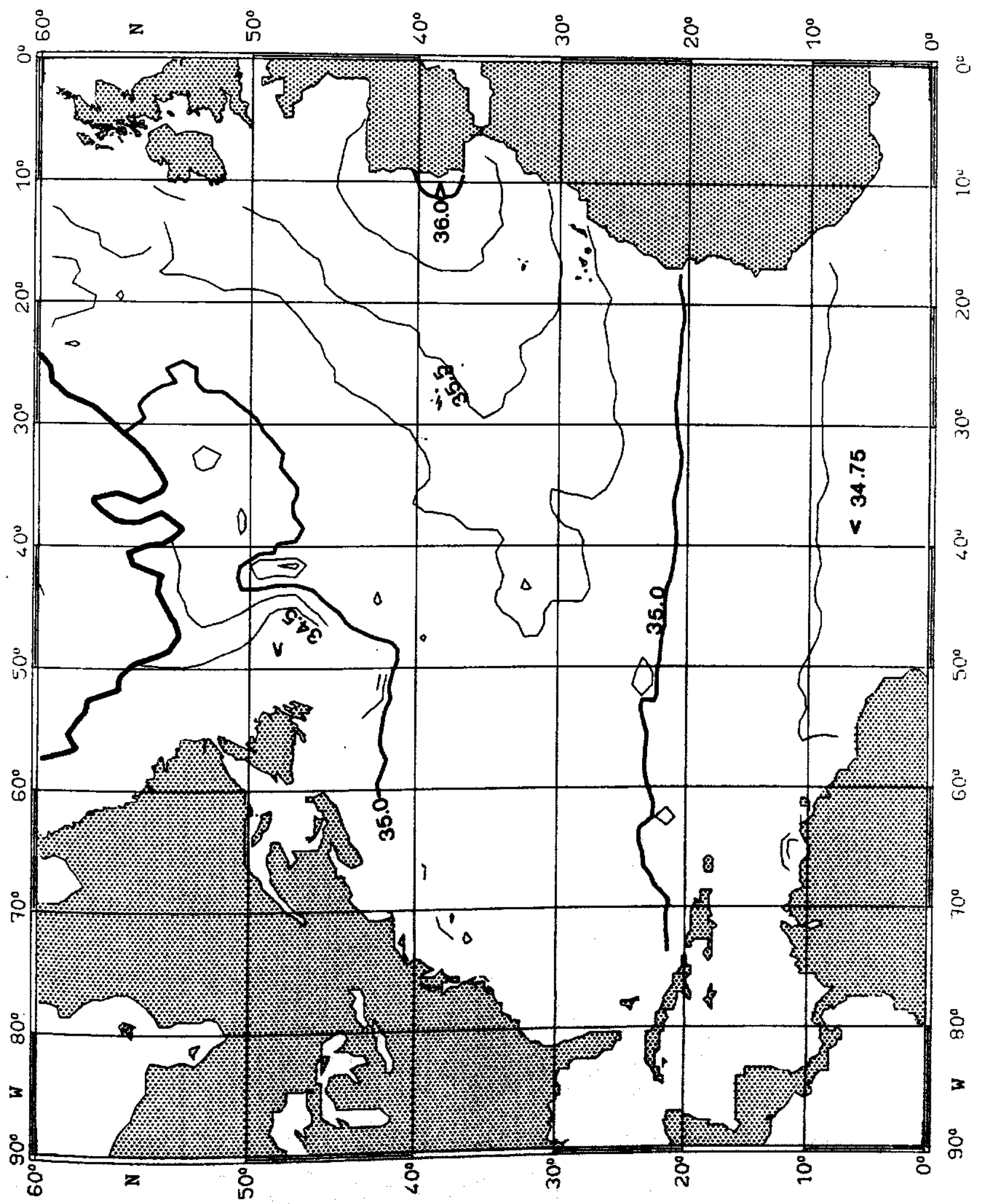


Fig. 25:

PRESSURE $\left(10^{4} \mathrm{~Pa}\right)$ on $\sigma_{\theta}=25.0 \mathrm{~kg} \mathrm{~m}^{-3} \quad$ MARCH

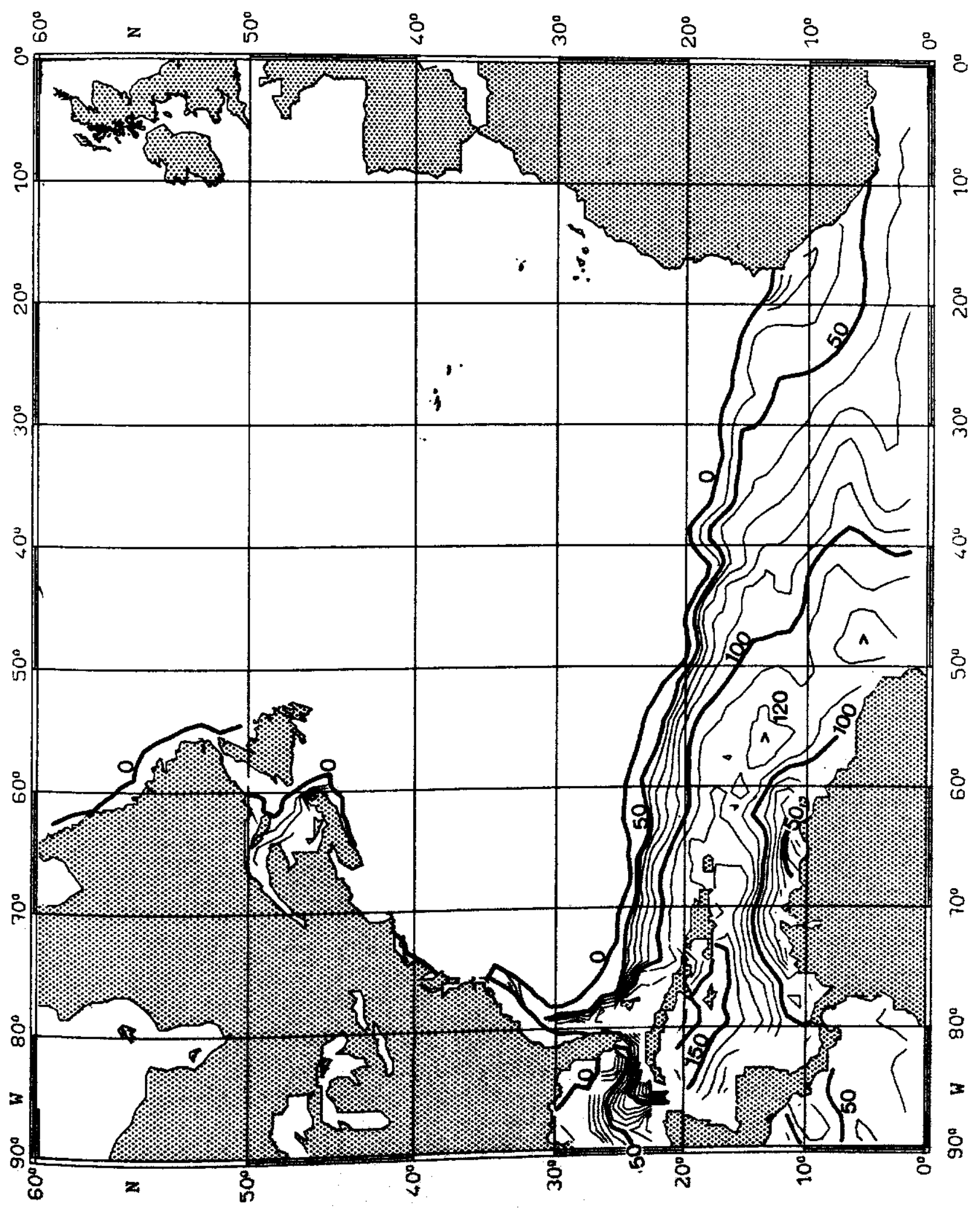


Fig. 26:

TEMPERATURE $\left({ }^{\circ} \mathrm{C}\right)$ on $\sigma_{\theta}=25.0 \mathrm{~kg} \mathrm{~m}^{-3}$

MARCH

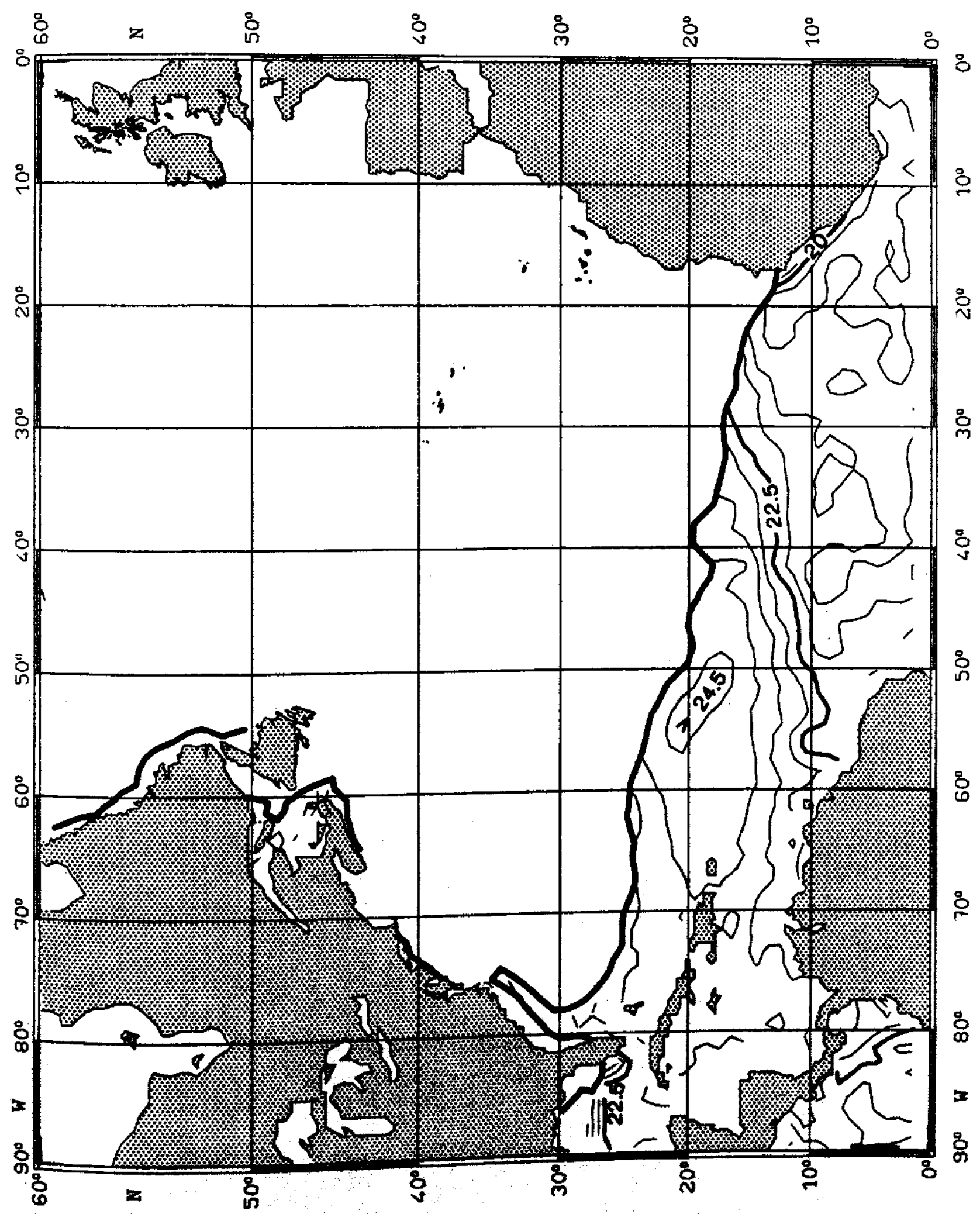


Fig. 27:

SALINITY $\left(10^{-3}\right)$ on $\sigma_{\theta}=25.0 \mathrm{~kg} \mathrm{~m}^{-3} \quad$ MARCH

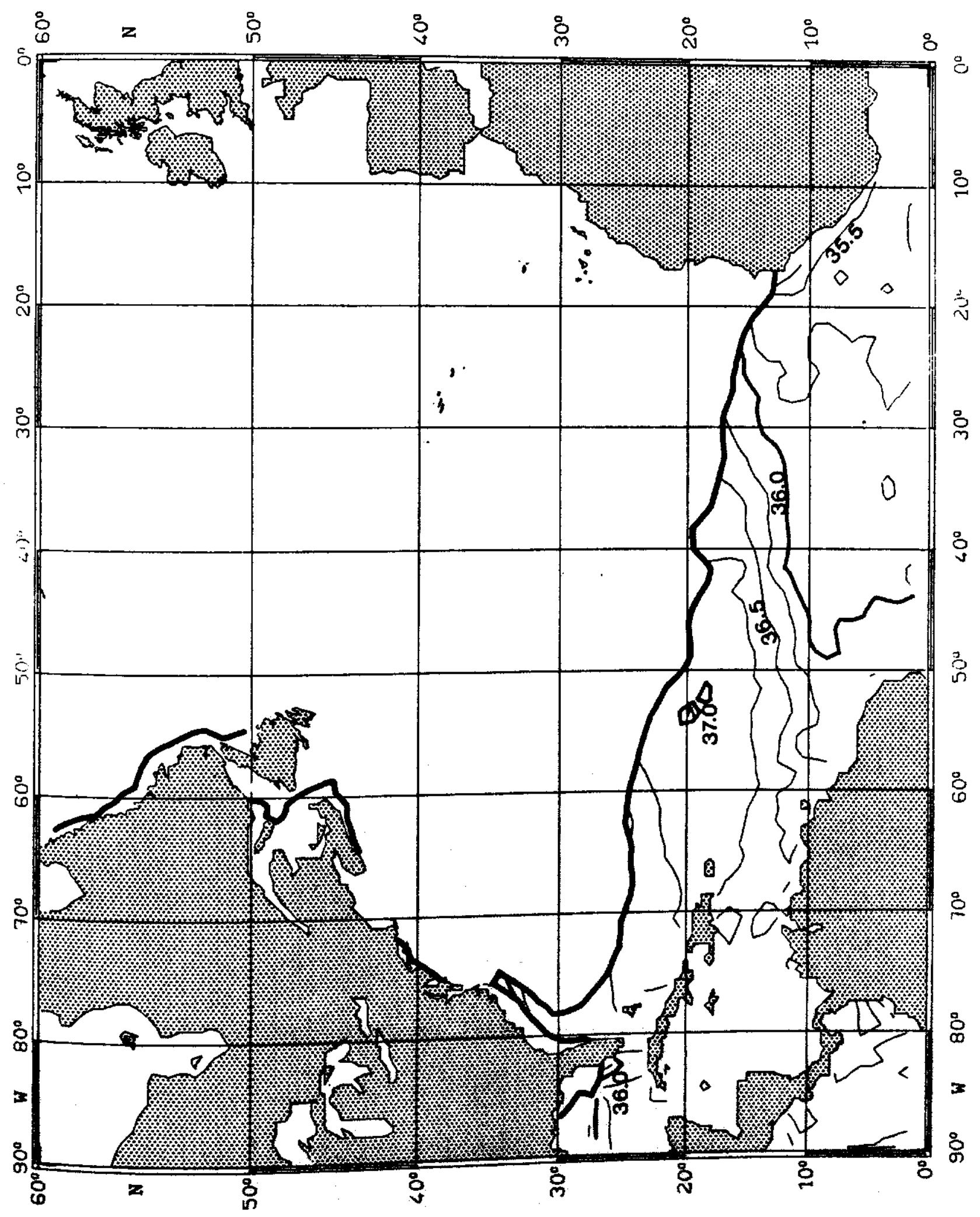


Fig. 28:

PRESSURE $\left(10^{4} \mathrm{~Pa}\right)$ on $\sigma_{\theta}=26.0 \mathrm{~kg} \mathrm{~m}^{-3}$

MARCH

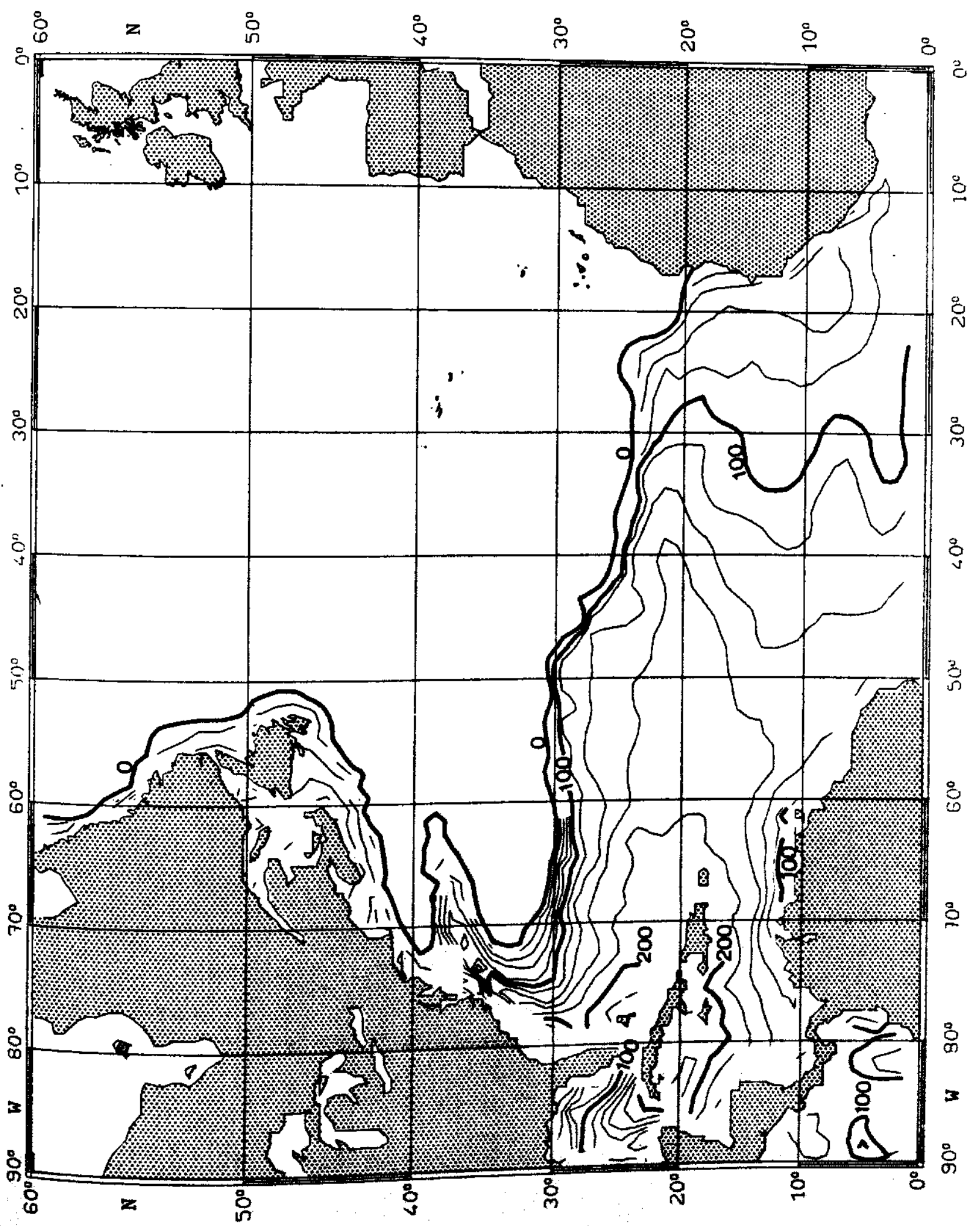


Fig. 29:

TEMPERATURE $\left({ }^{\circ} \mathrm{C}\right)$ on $\sigma_{\theta}=26.0 \mathrm{~kg} \mathrm{~m}^{-3} \quad$ MARCH

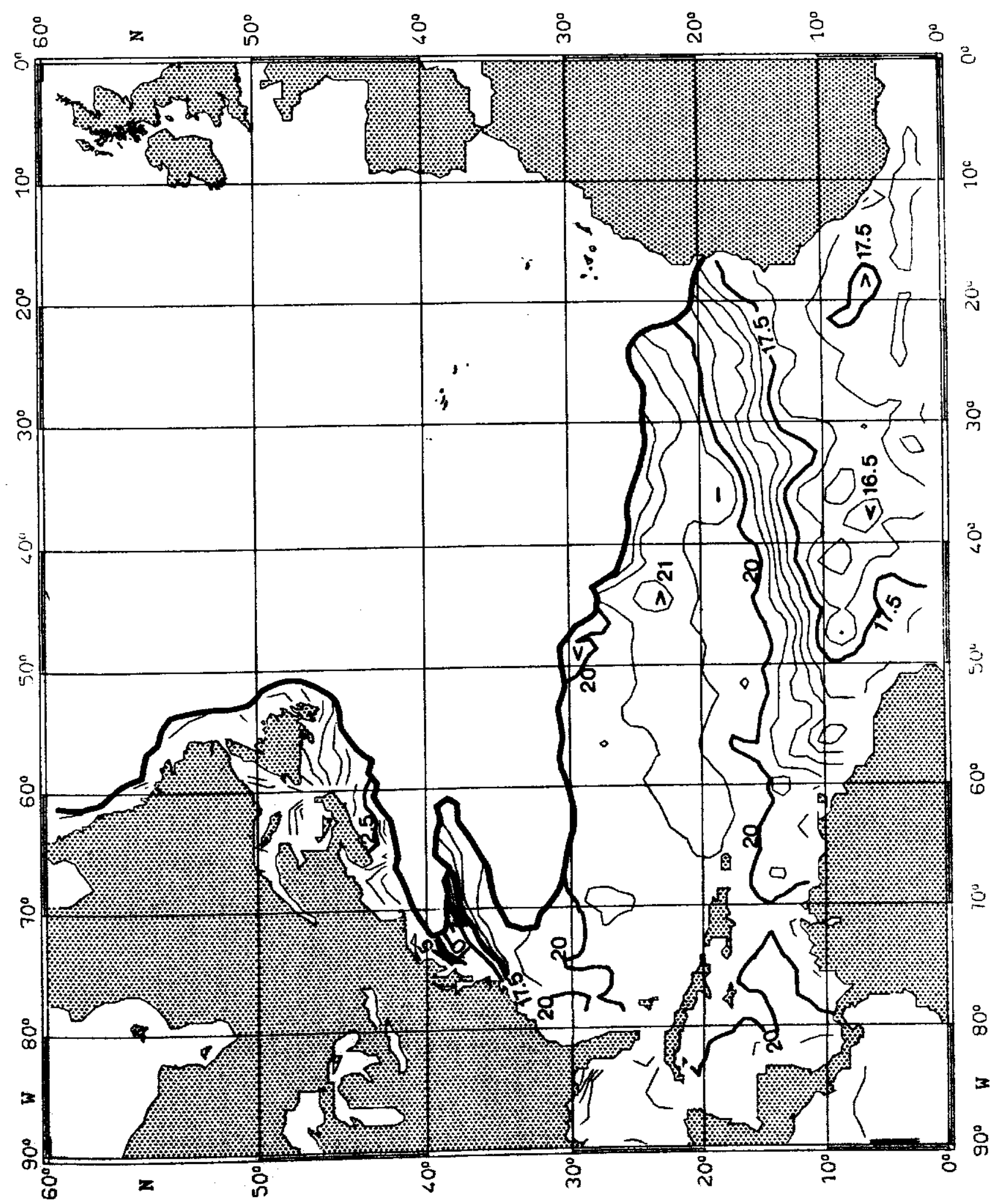


Fig. 30:

SALINITY $\left(10^{-3}\right)$ on $\sigma_{\theta}=26.0 \mathrm{~kg} \mathrm{~m}^{-3} \quad$ MARCH

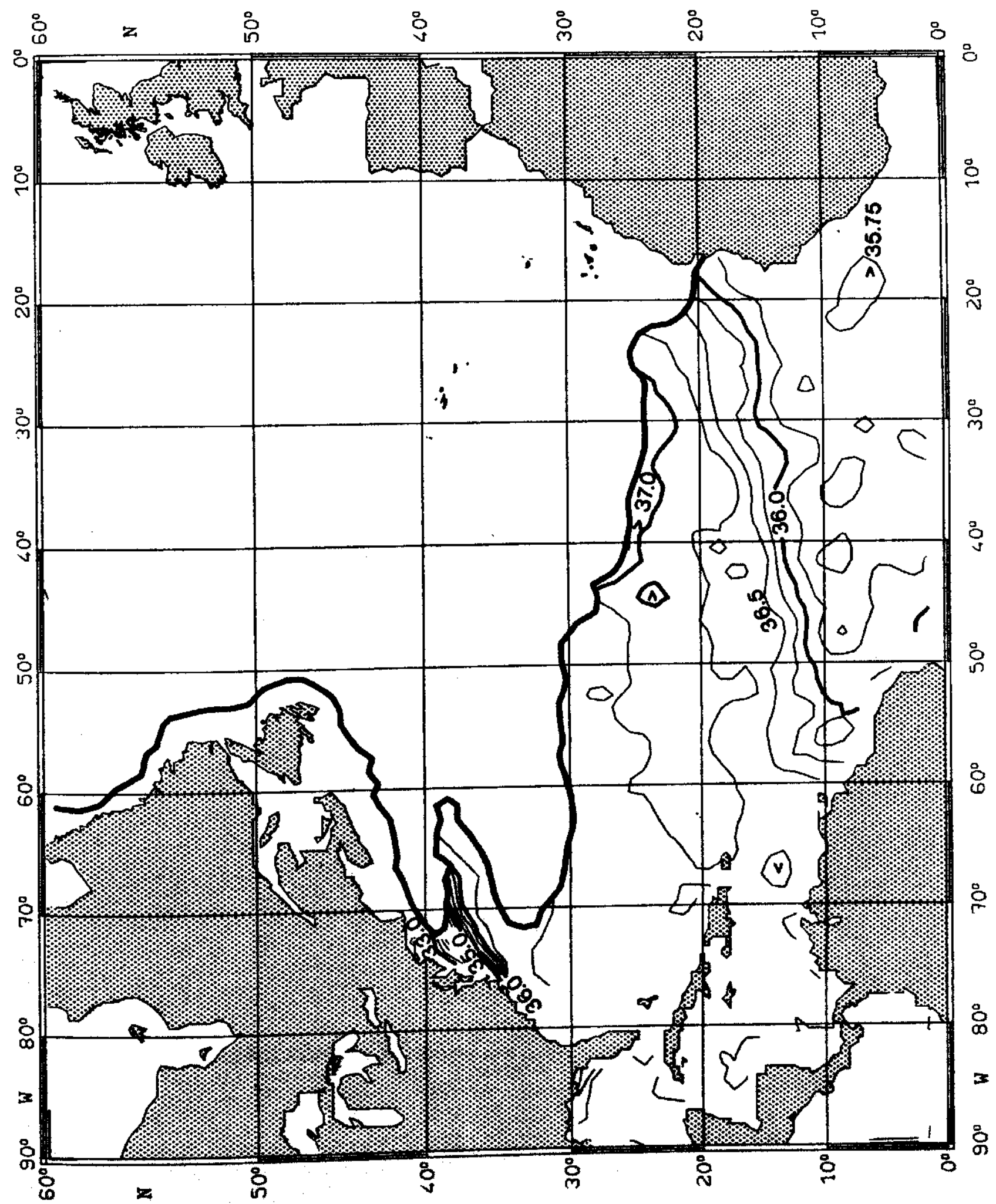


Fig. 31:

PRESSURE $\left(10^{4} \mathrm{~Pa}\right)$ on $\sigma_{\theta}=27.0 \mathrm{~kg} \mathrm{~m}^{-3} \quad$ MARCH

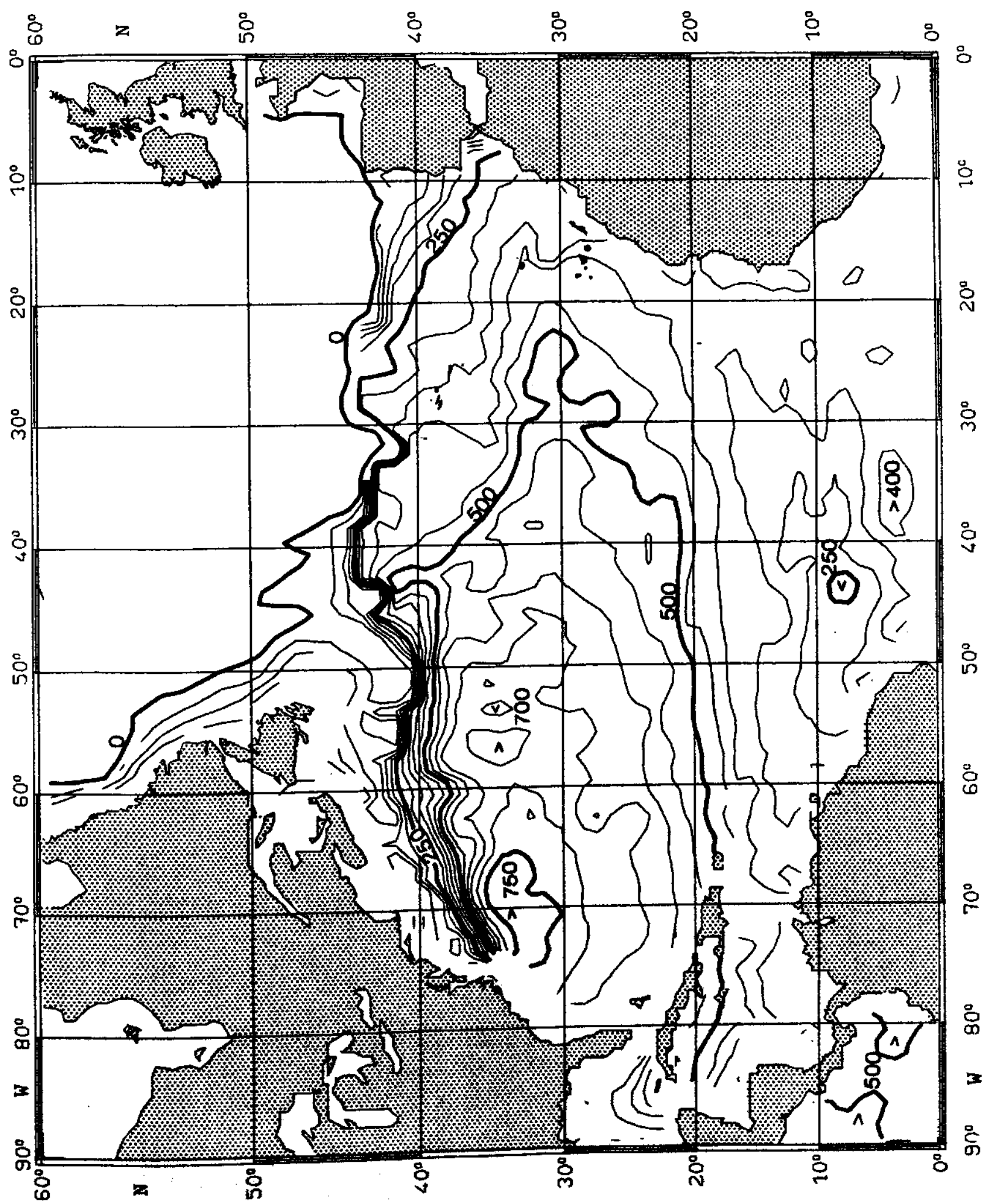


Fig. 32:

TEMPERATURE $\left({ }^{\circ} \mathrm{C}\right)$ on $\sigma_{\theta}=27.0 \mathrm{~kg} \mathrm{~m}^{-3} \quad$ MARCH

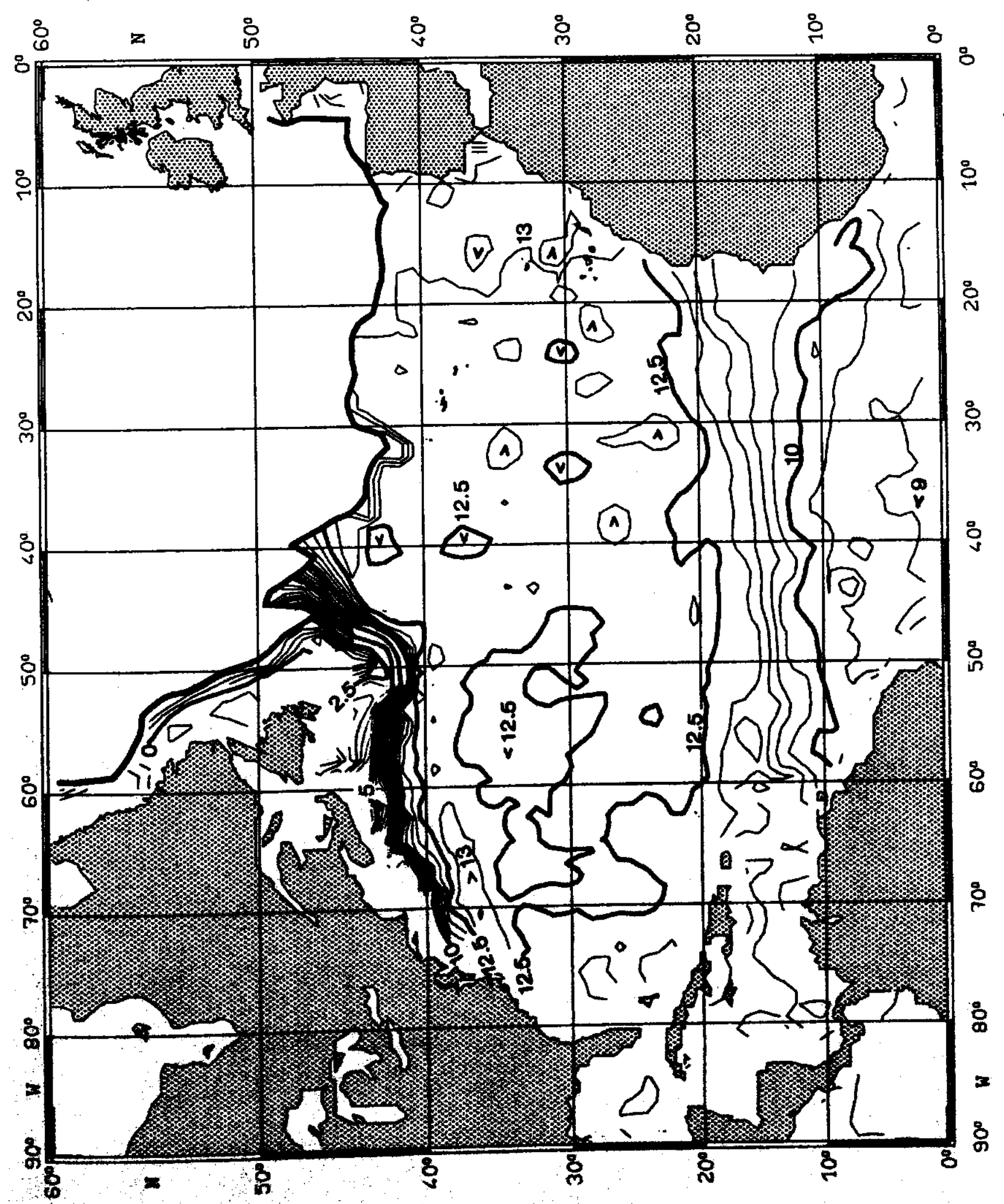


Fig. 33:

SALINITY $\left(10^{-3}\right)$ on $\sigma_{\theta}=27.0 \mathrm{~kg} \mathrm{~m}^{-3} \quad$ MARCH

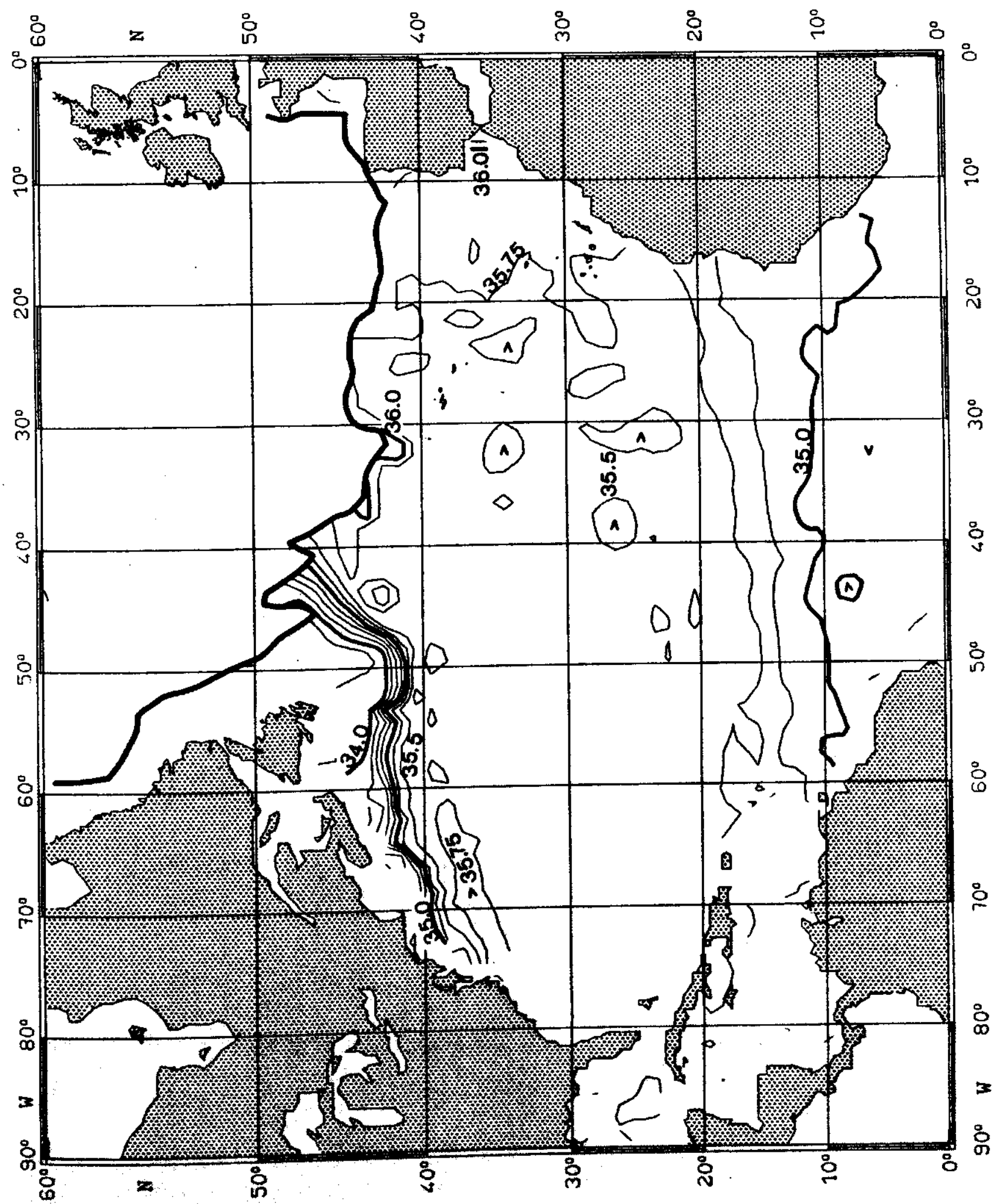


Fig. 34 :

PRESSURE $\left(10^{4} \mathrm{~Pa}\right)$ on $\sigma_{\theta}=27.5 \mathrm{~kg} \mathrm{~m}^{-3}$

MARCH

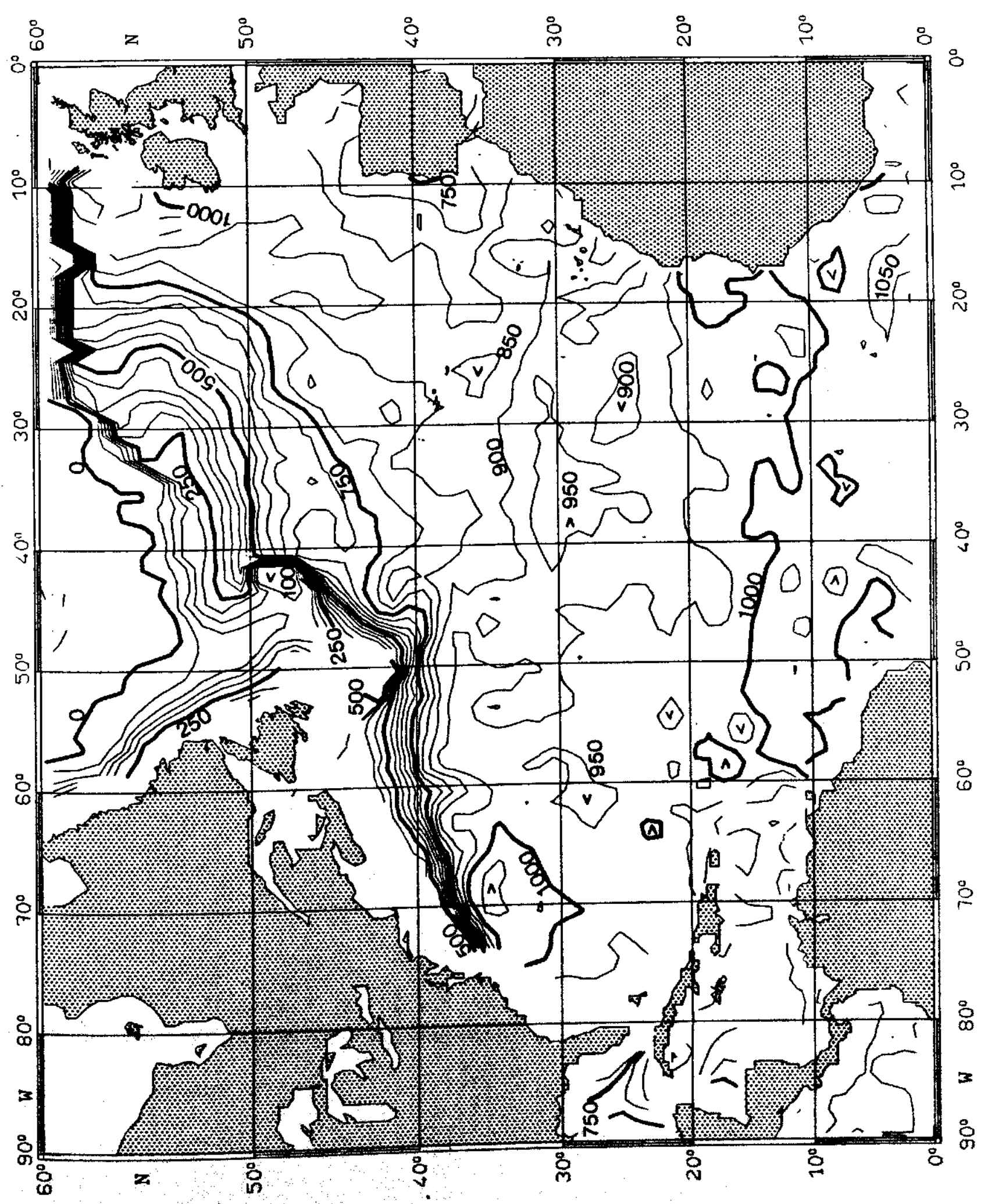


Fig. 35:

TEMPERATURE $\left({ }^{\circ} \mathrm{C}\right)$ on $\sigma_{\theta}=27.5 \mathrm{~kg} \mathrm{~m}^{-3} \quad$ MARCH

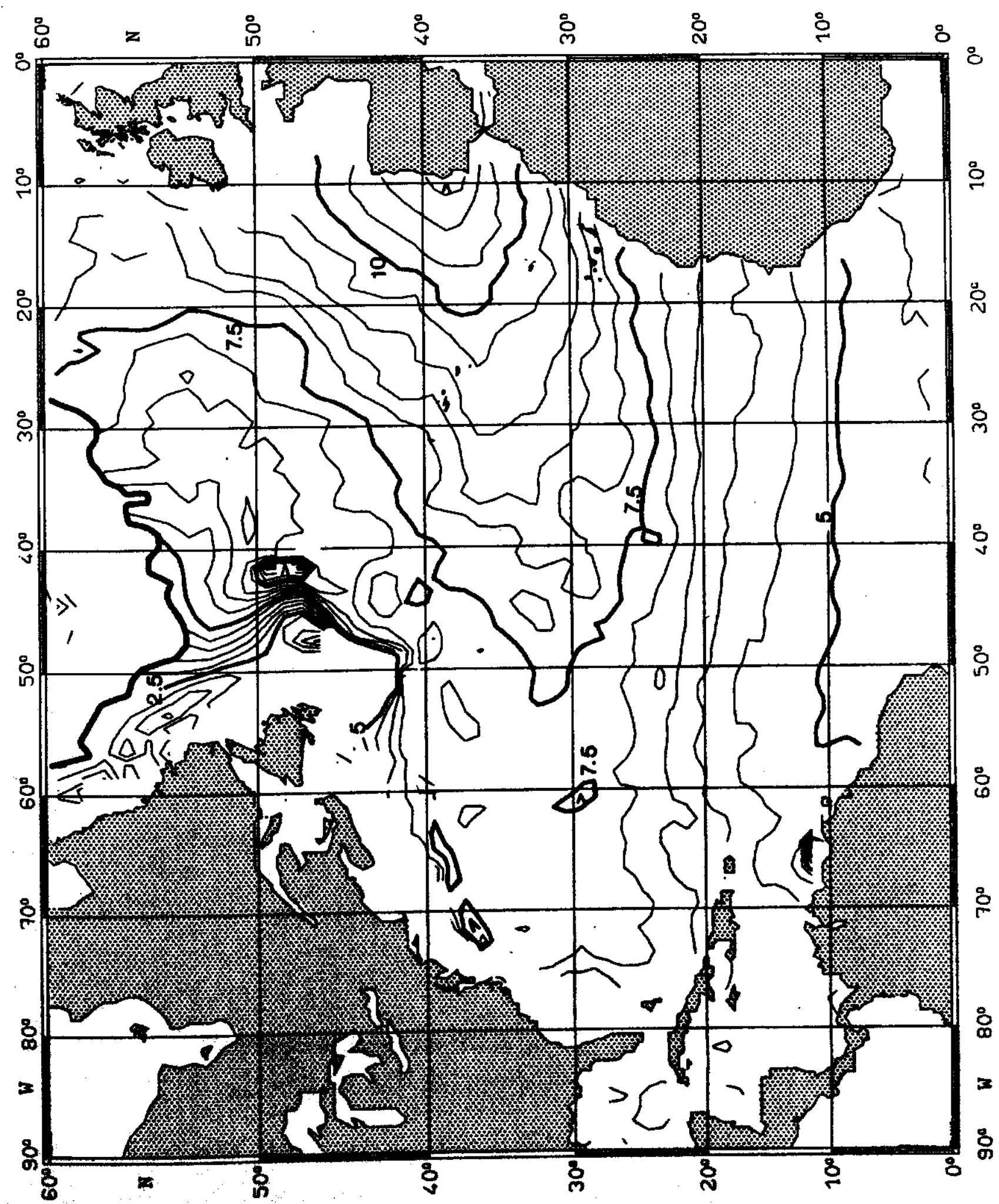


Fig. 36:

SALINITY $\left(10^{-3}\right)$ on $\sigma_{\theta}=27.5 \mathrm{~kg} \mathrm{~m}^{-3} \quad$ MARCH

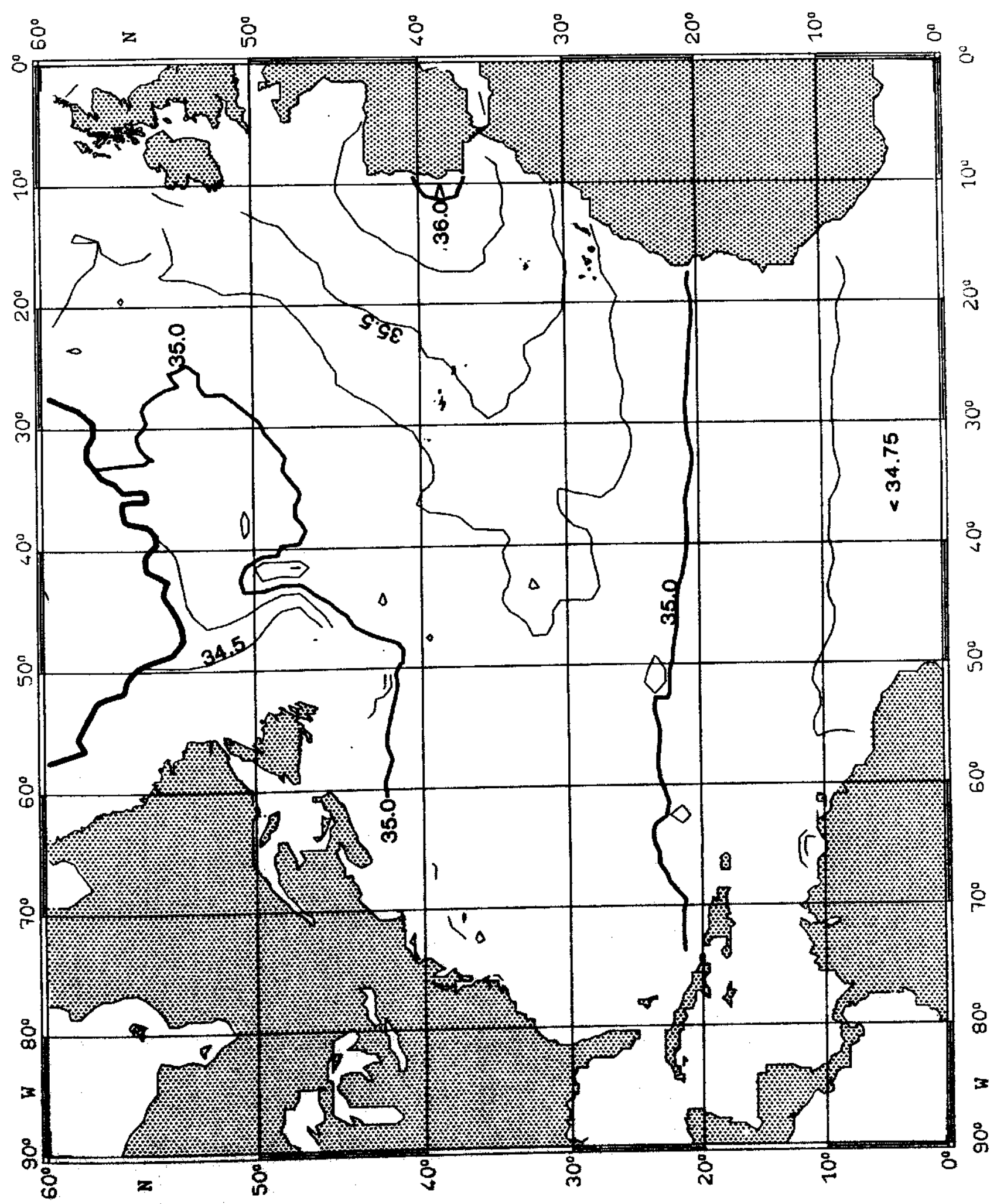


Fig. 37:

PRESSURE $\left(10^{4} \mathrm{~Pa}\right)$ on $\sigma_{\theta}=25.0 \mathrm{~kg} \mathrm{~m}^{-3} \quad$ APRIL

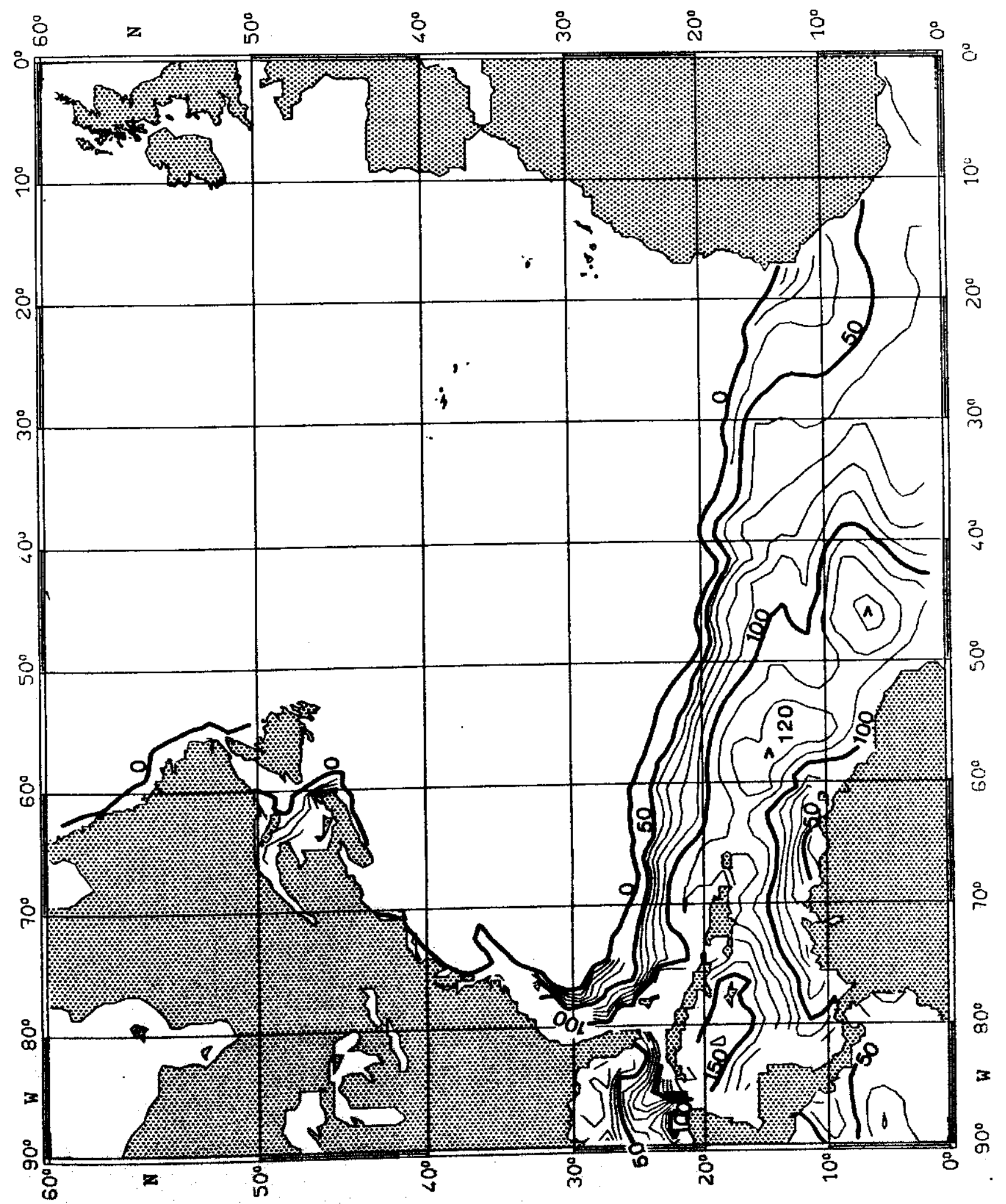


Fig. 38:

TEMPERATURE $\left({ }^{\circ} \mathrm{C}\right)$ on $\sigma_{\theta}=25.0 \mathrm{~kg} \mathrm{~m} \quad$ APRIL

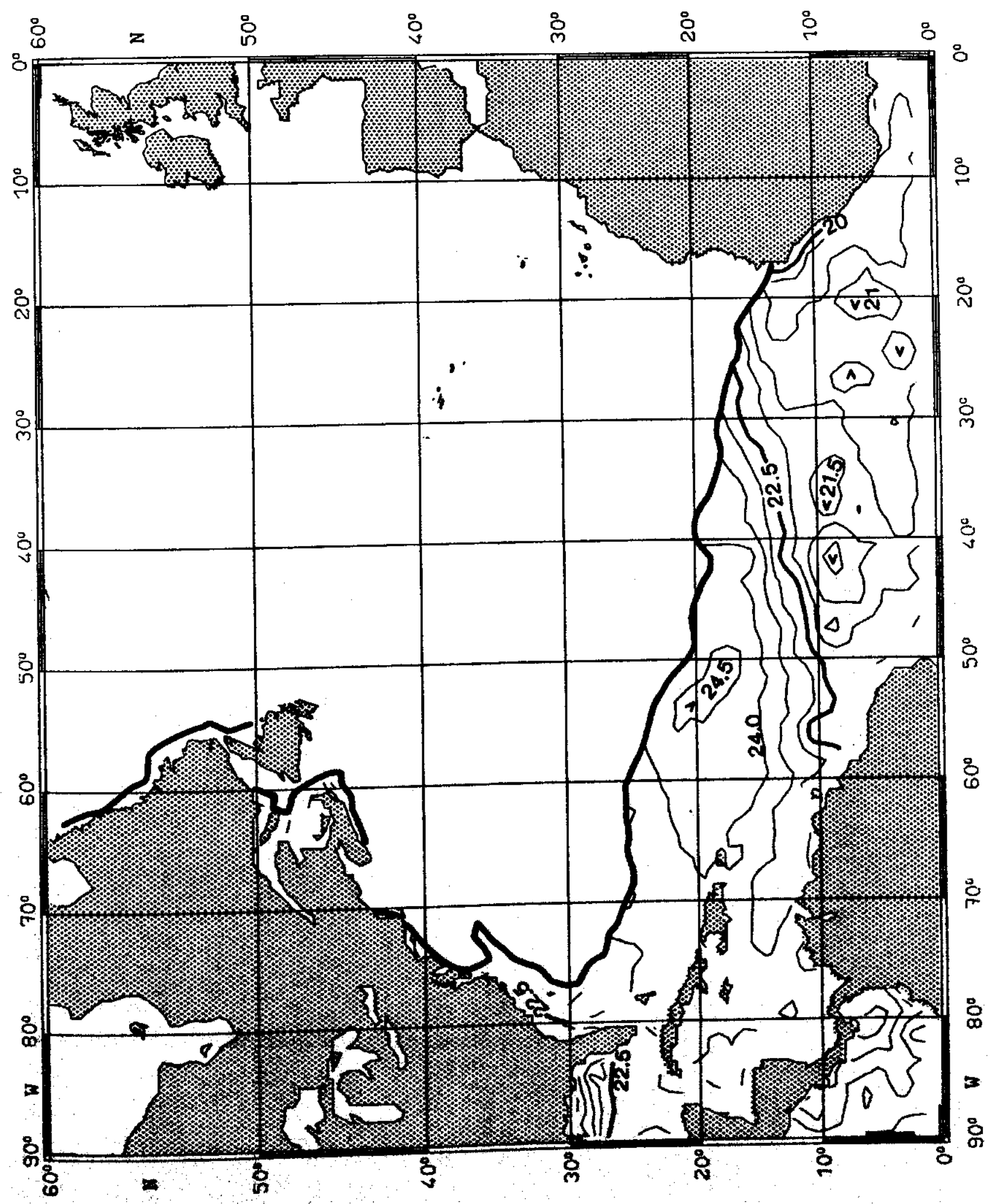


Fig. 39:

SALINITY $\left(10^{-3}\right)$ on $\sigma_{\theta}=25.0 \mathrm{~kg} \mathrm{~m}^{-3} \quad$ APRIL

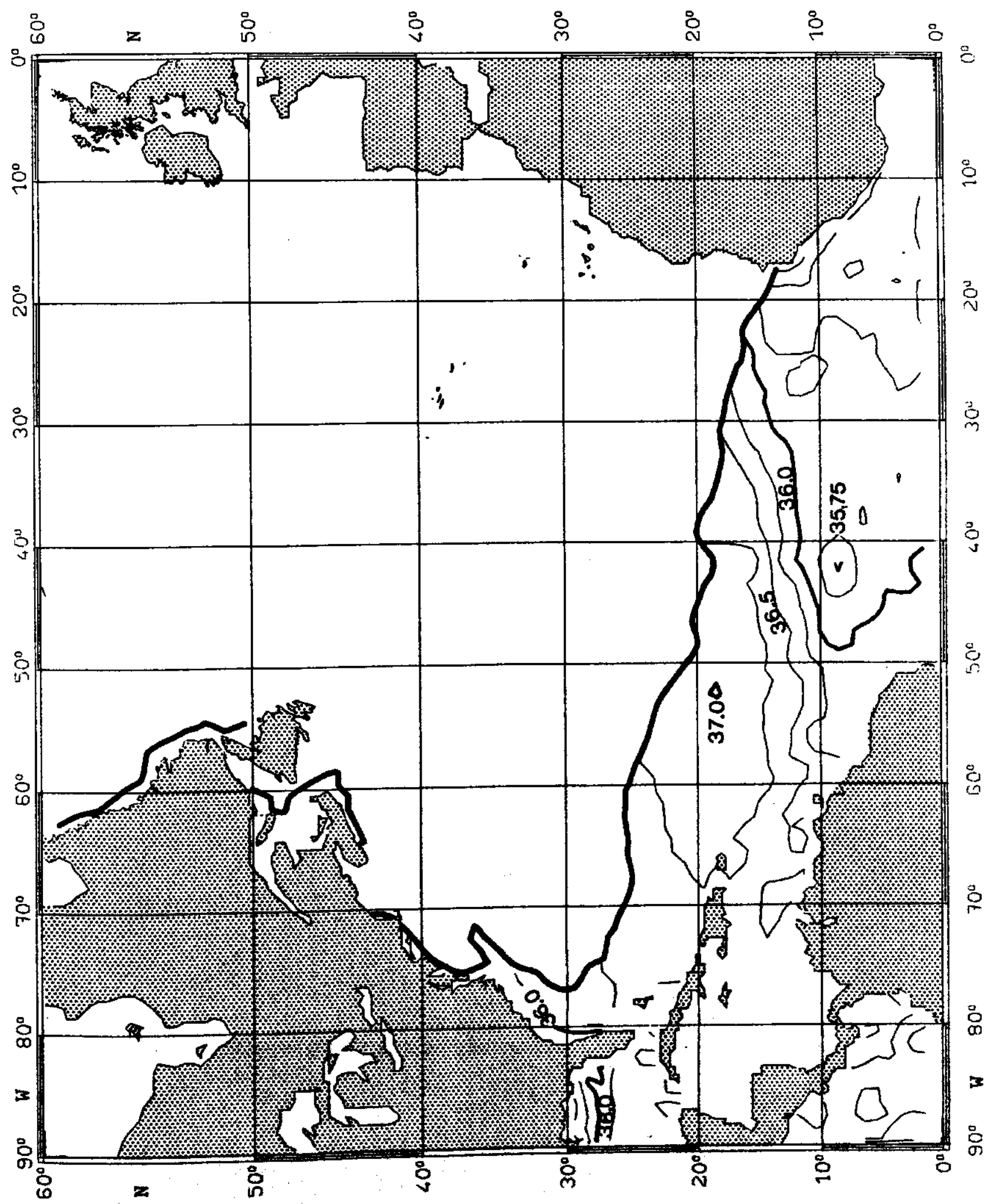


Fig. 40:

PRESSURE $\left(10^{4} \mathrm{~Pa}\right)$ on $\sigma_{\theta}=26.0 \mathrm{~kg} \mathrm{~m}^{-3} \quad$ APRIL

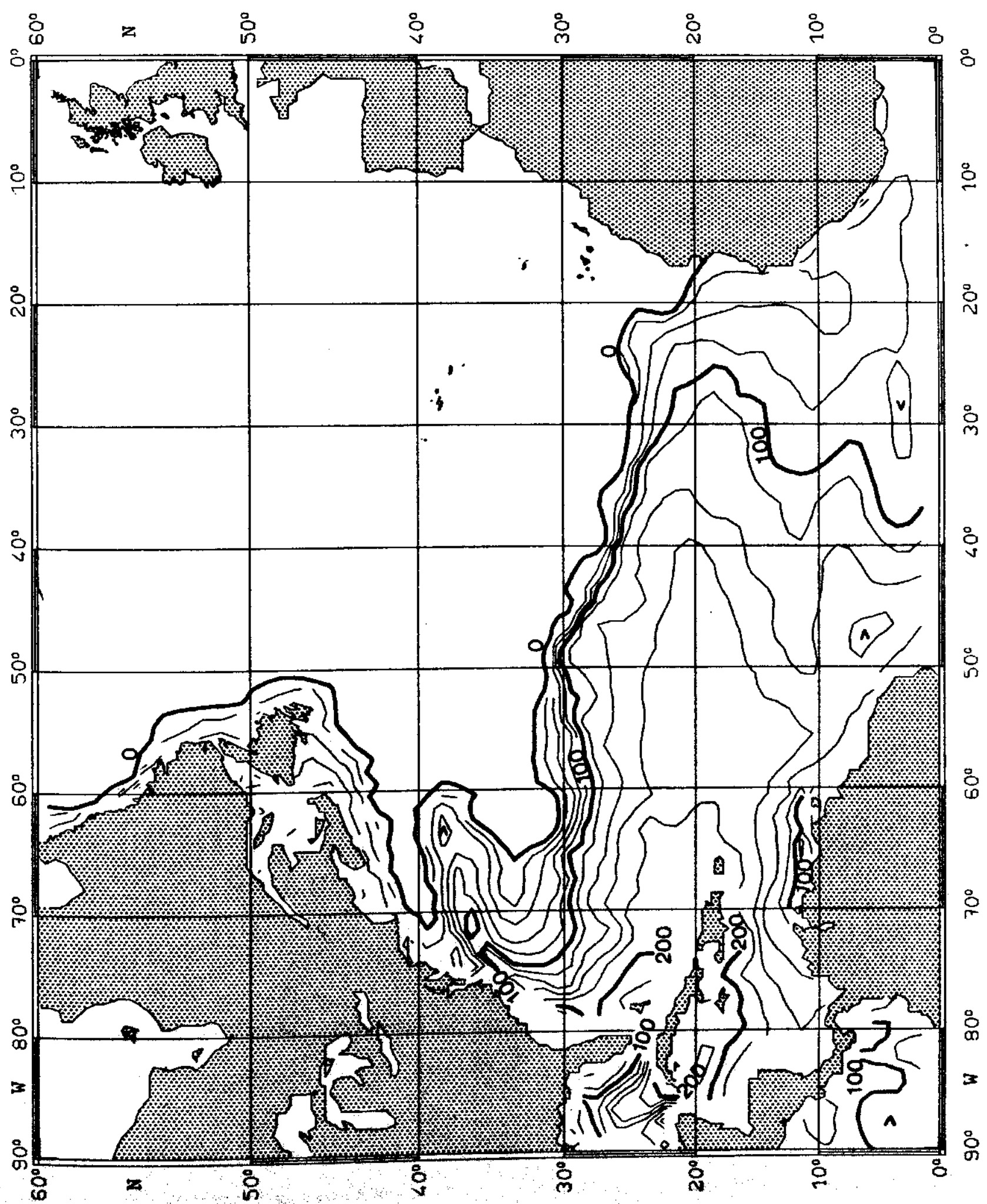


Fig. 41:

TEMPERATURE $\left({ }^{\circ} \mathrm{C}\right)$ on $\sigma_{\theta}=26.0 \mathrm{~kg} \mathrm{~m}^{-3} \quad$ APRIL

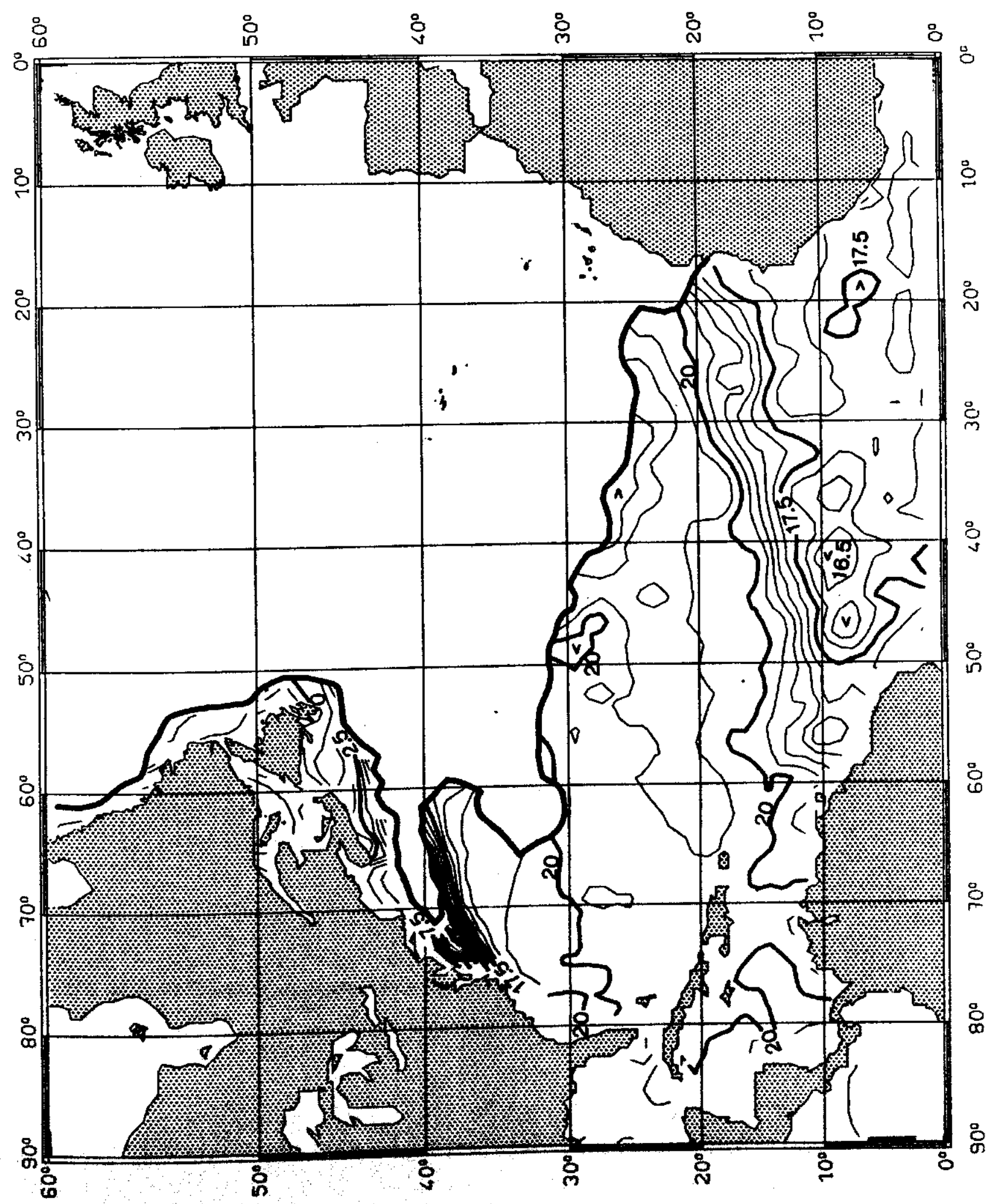


Fig. 42:

SALINITY $\left(10^{-3}\right)$ on $\sigma_{\theta}=26.0 \mathrm{~kg} \mathrm{~m}^{-3} \quad$ APRIL

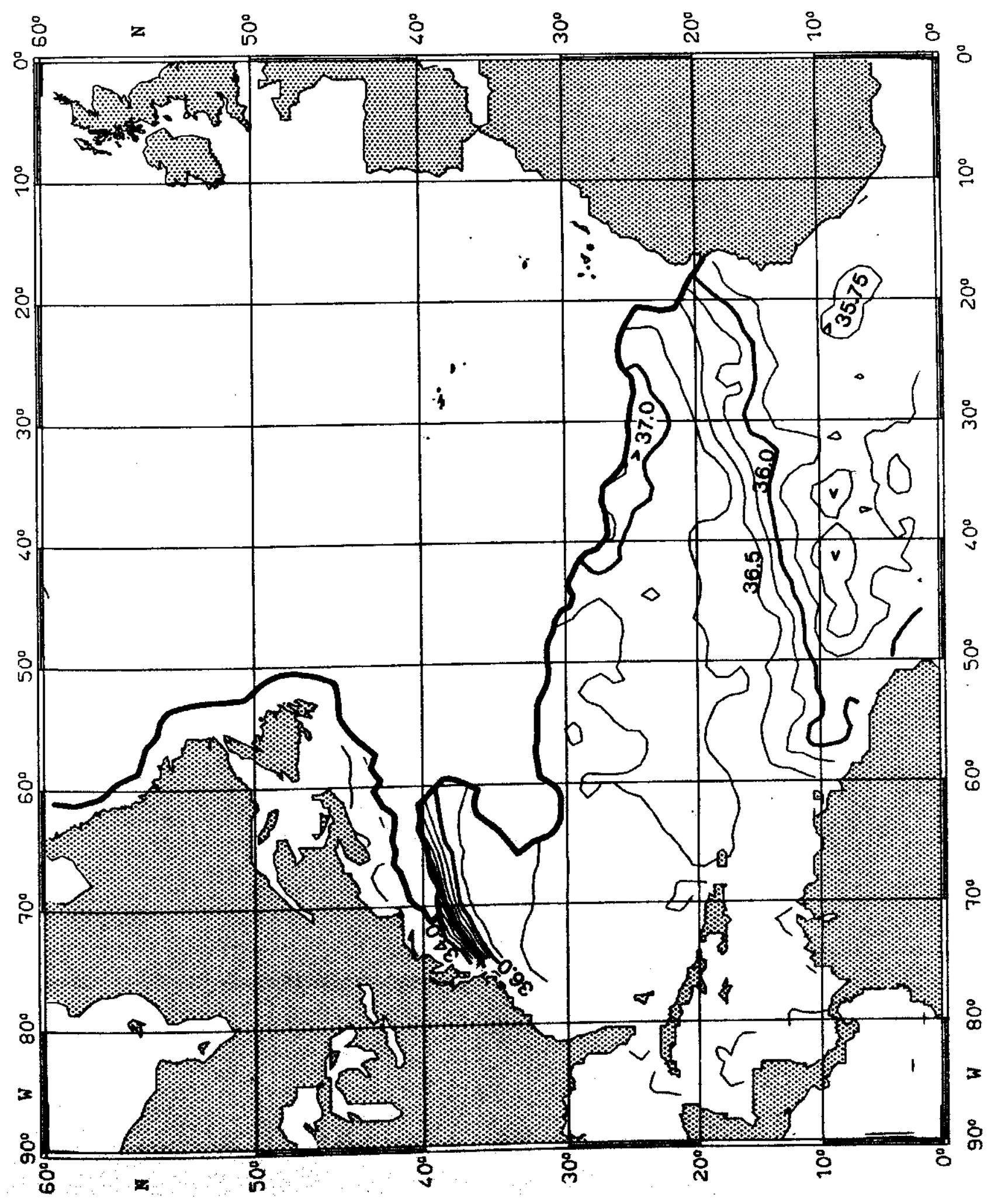


Fig. 43:

PRESSURE $\left(10^{4} \mathrm{~Pa}\right)$ on $\sigma_{\theta}=27.0 \mathrm{~kg} \mathrm{~m}^{-3} \quad$ APRIL

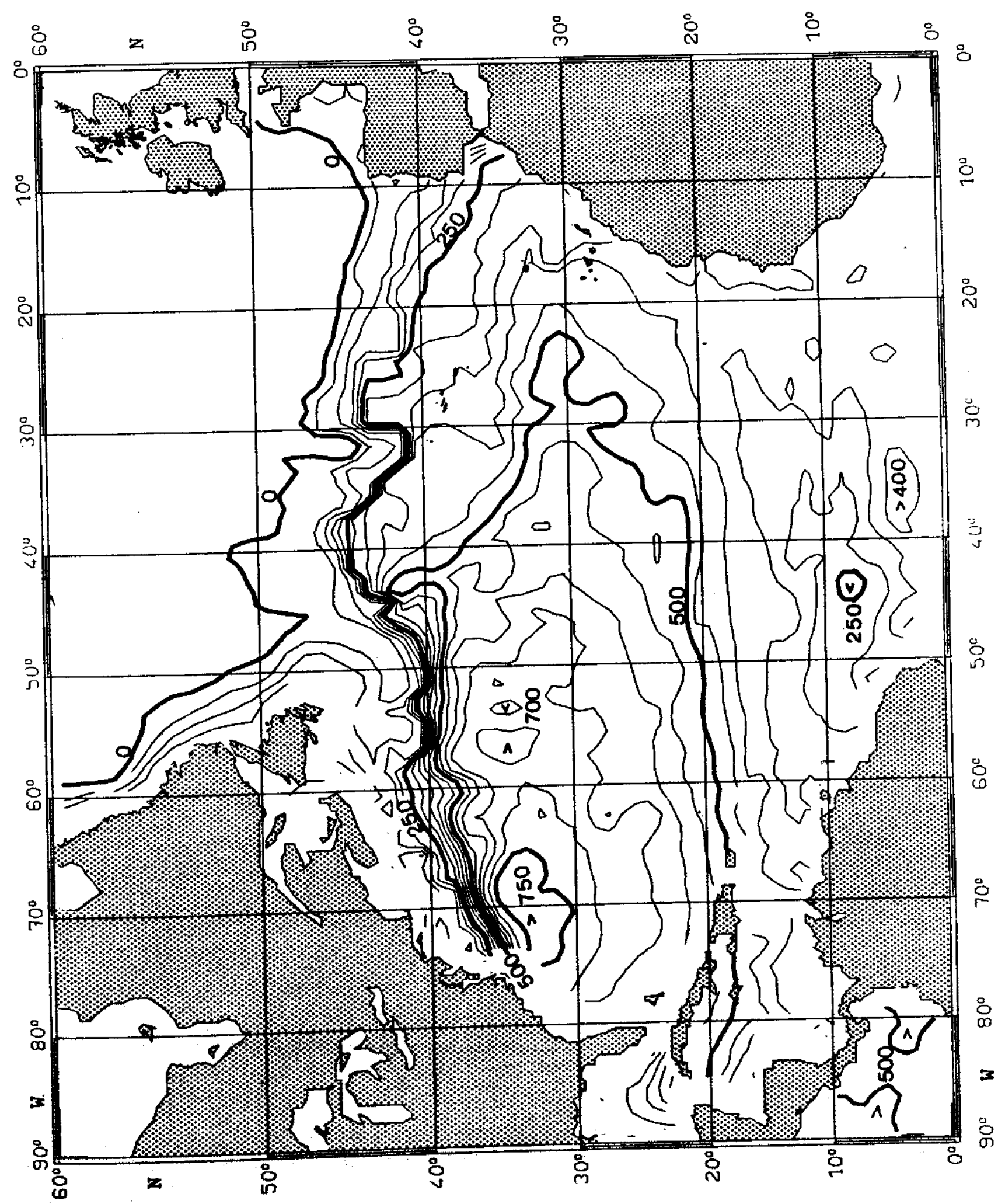


Fig. 44:

TEMPERATURE $\left({ }^{\circ} \mathrm{C}\right)$ on $\sigma_{\theta}=27.0 \mathrm{~kg} \mathrm{~m}^{-3} \quad$ APRIL

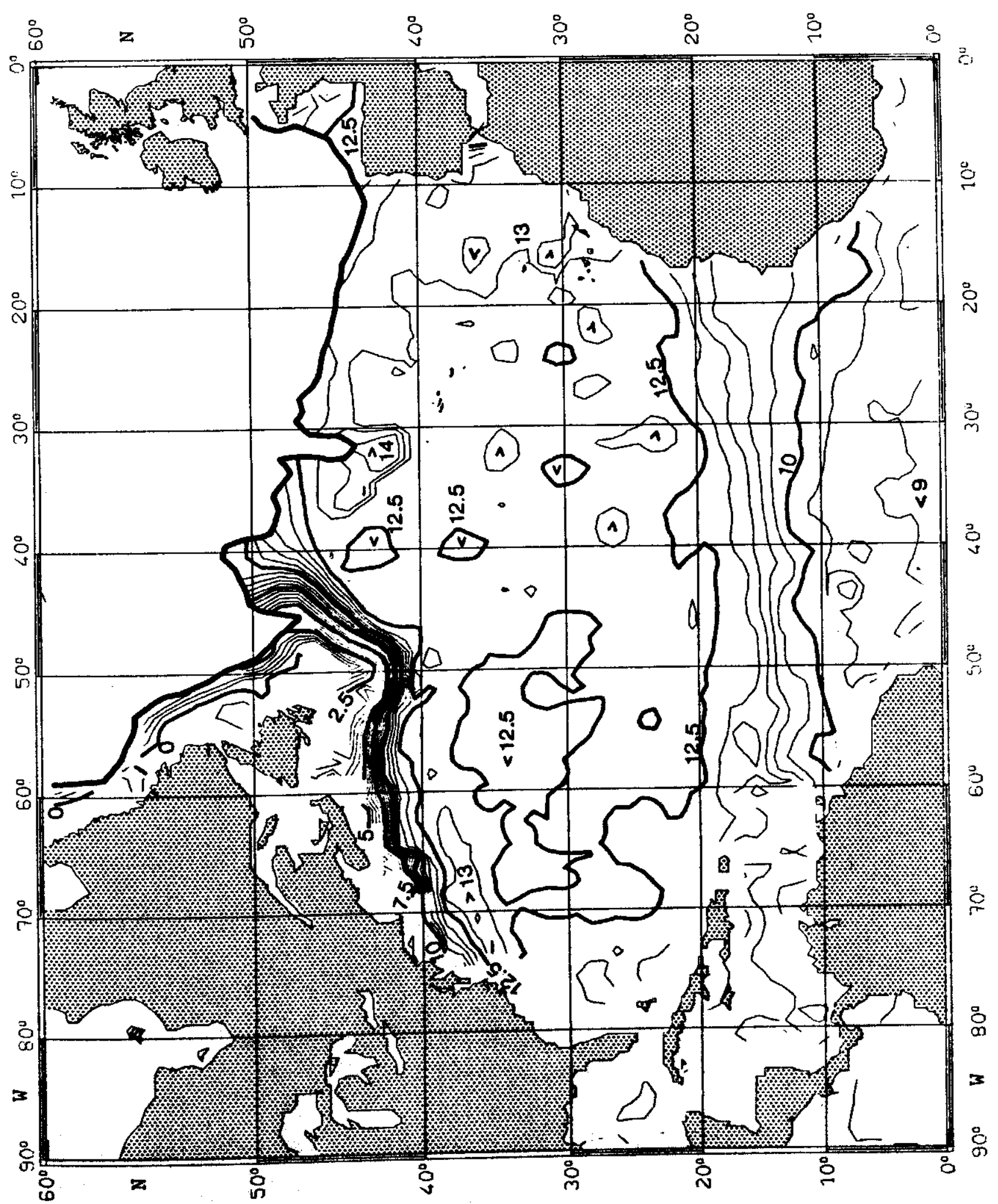


Fig. 45:

SALINITY $\left(10^{-3}\right)$ on $\sigma_{\theta}=27.0 \mathrm{~kg} \mathrm{~m}^{-3} \quad$ APRIL

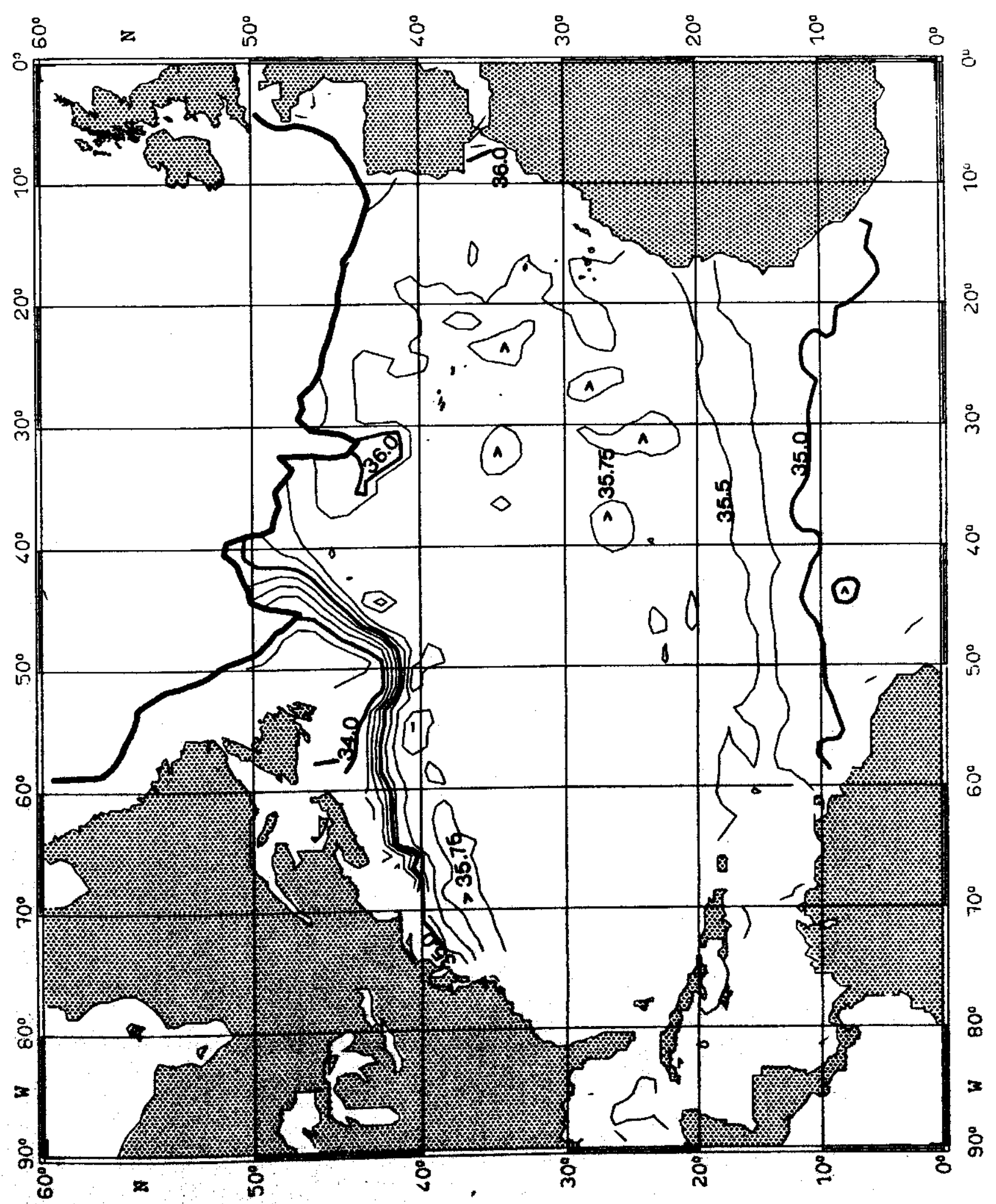


Fig. 46:

PRESSURE $\left(10^{4} \mathrm{~Pa}\right)$ on $\sigma_{\theta}=25.0 \mathrm{~kg} \mathrm{~m}^{-3}$ MAY

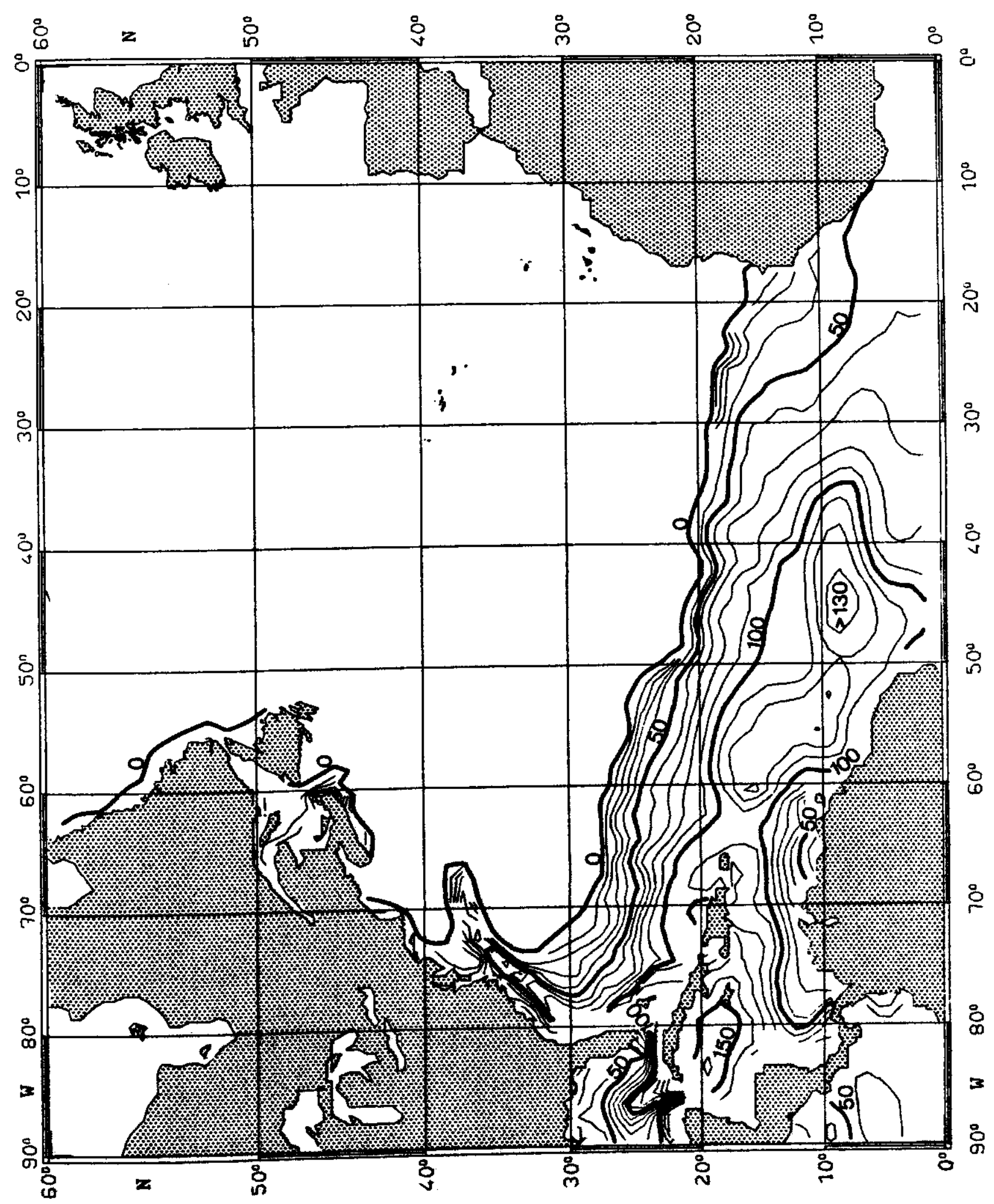


Fig. 47 :

TEMPERATURE $\left({ }^{\circ} \mathrm{C}\right)$ on $\sigma_{\theta}=25.0 \mathrm{~kg} \mathrm{~m}^{-3}$ MAY

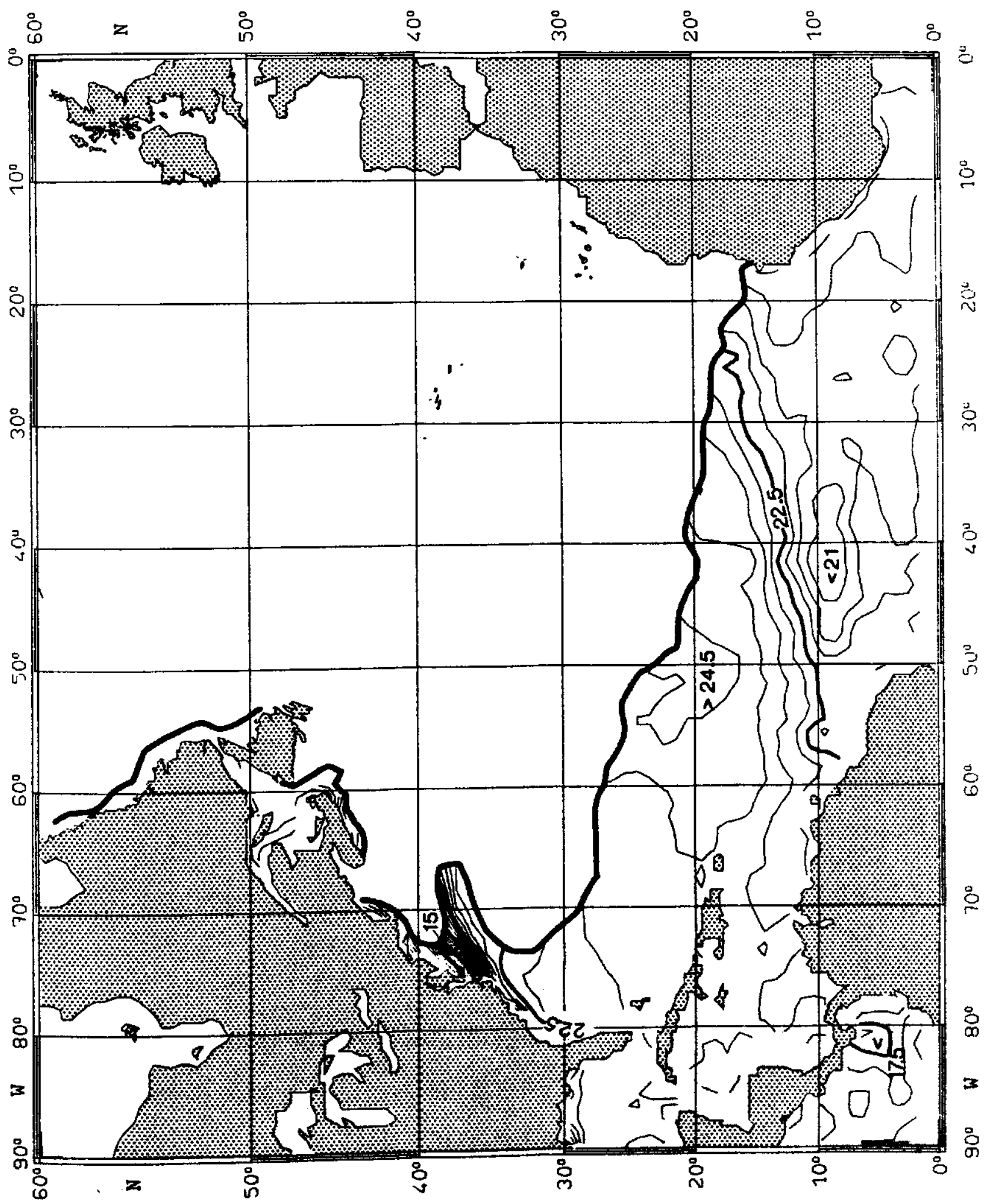


Fig. 48:

SALINITY $\left(10^{-3}\right)$ on $\sigma_{\theta}=25.0 \mathrm{~kg} \mathrm{~m}^{-3} \quad$ MAY

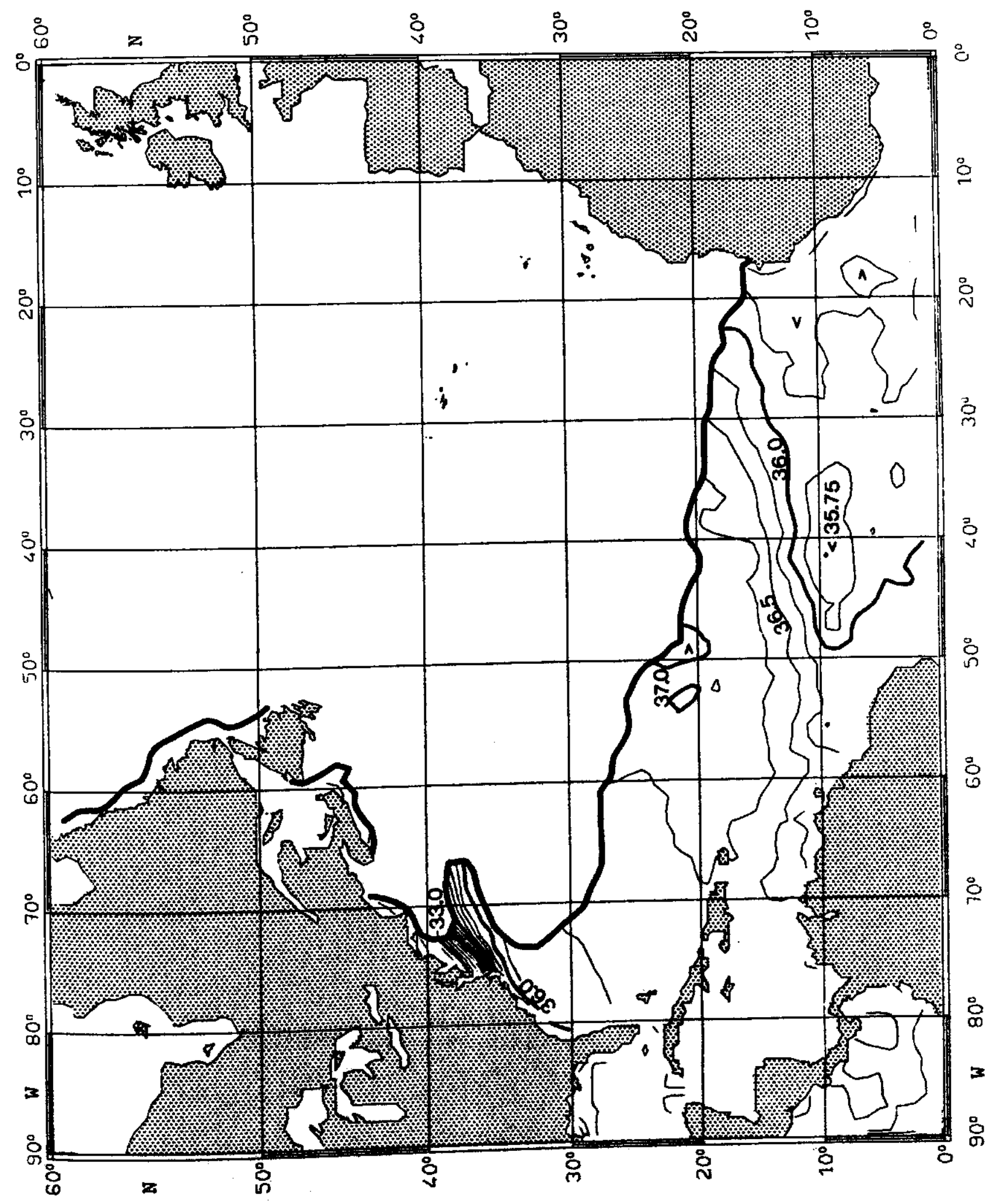


Fig. 49:

PRESSURE $\left(10^{4} \mathrm{~Pa}\right)$ on $\sigma_{\theta}=26.0 \mathrm{~kg} \mathrm{~m}^{-3} \quad$ MAY

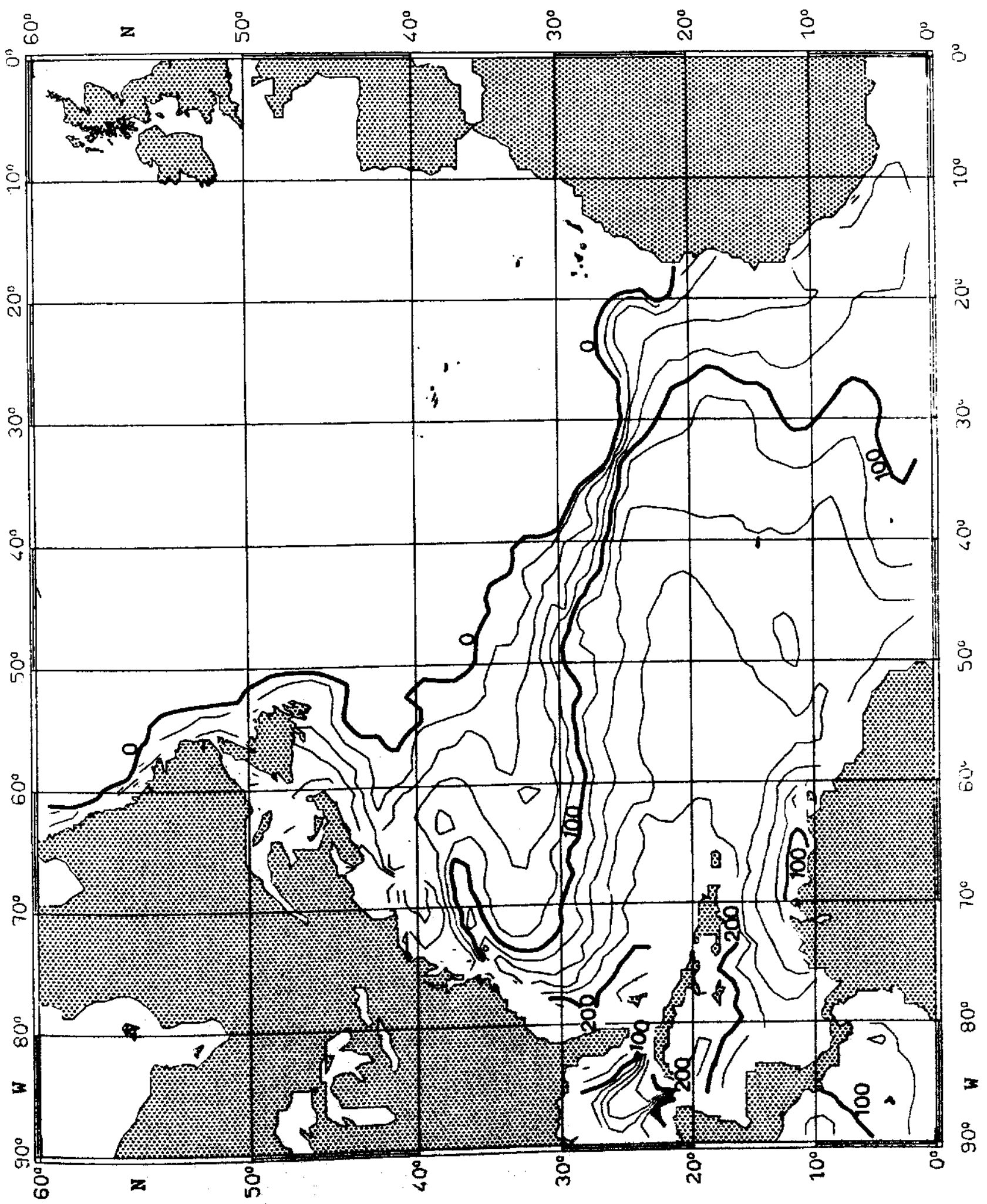


Fig. 50:

TEMPERATURE $\left({ }^{\circ} \mathrm{C}\right)$ on $\sigma_{\theta}=26.0 \mathrm{~kg} \mathrm{~m}^{-3}$

MAY

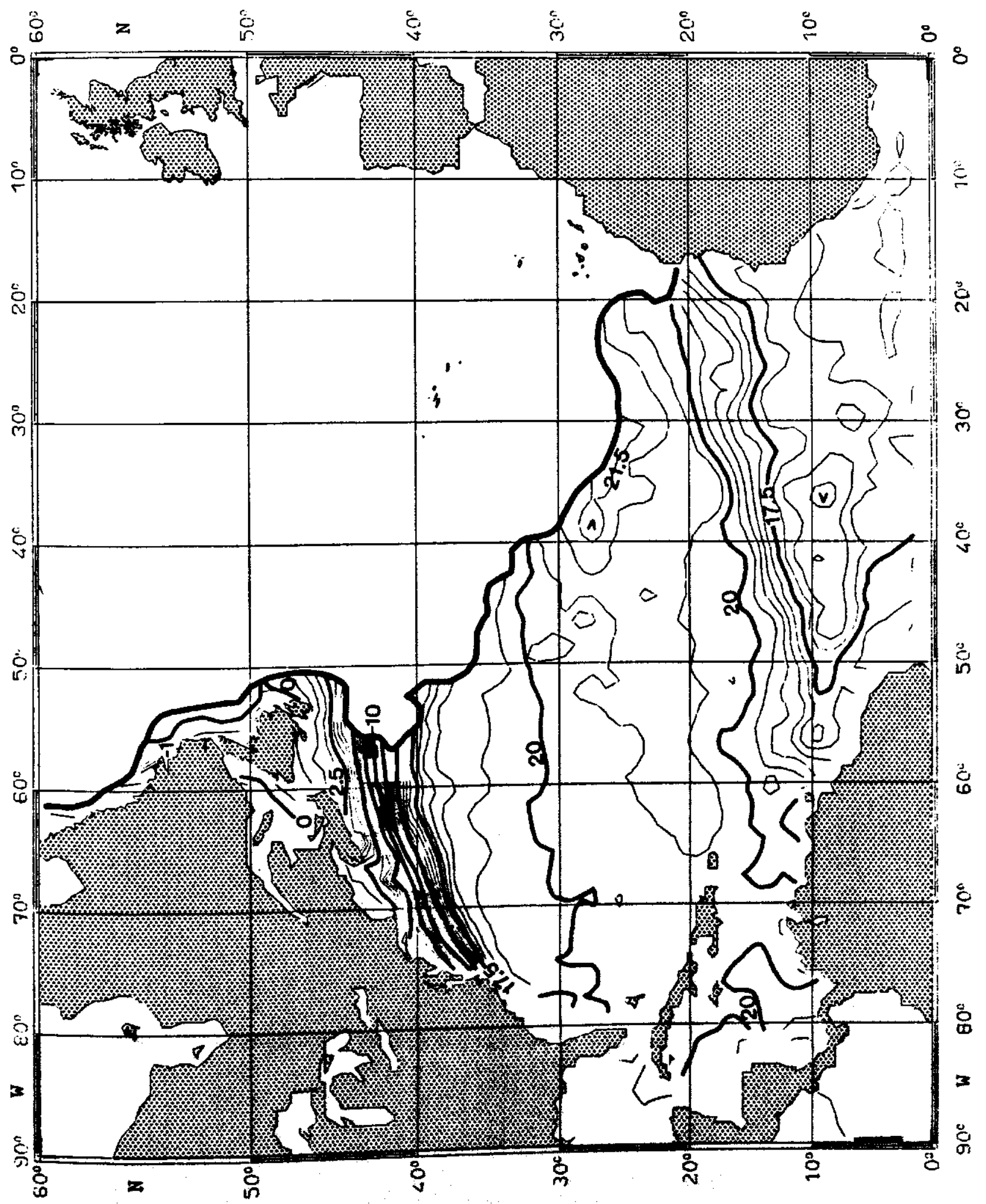


Fig. 51:

SALINITY $\left(10^{-3}\right)$ on $\sigma_{\theta}=26.0 \mathrm{~kg} \mathrm{~m}^{-3}$

MAY

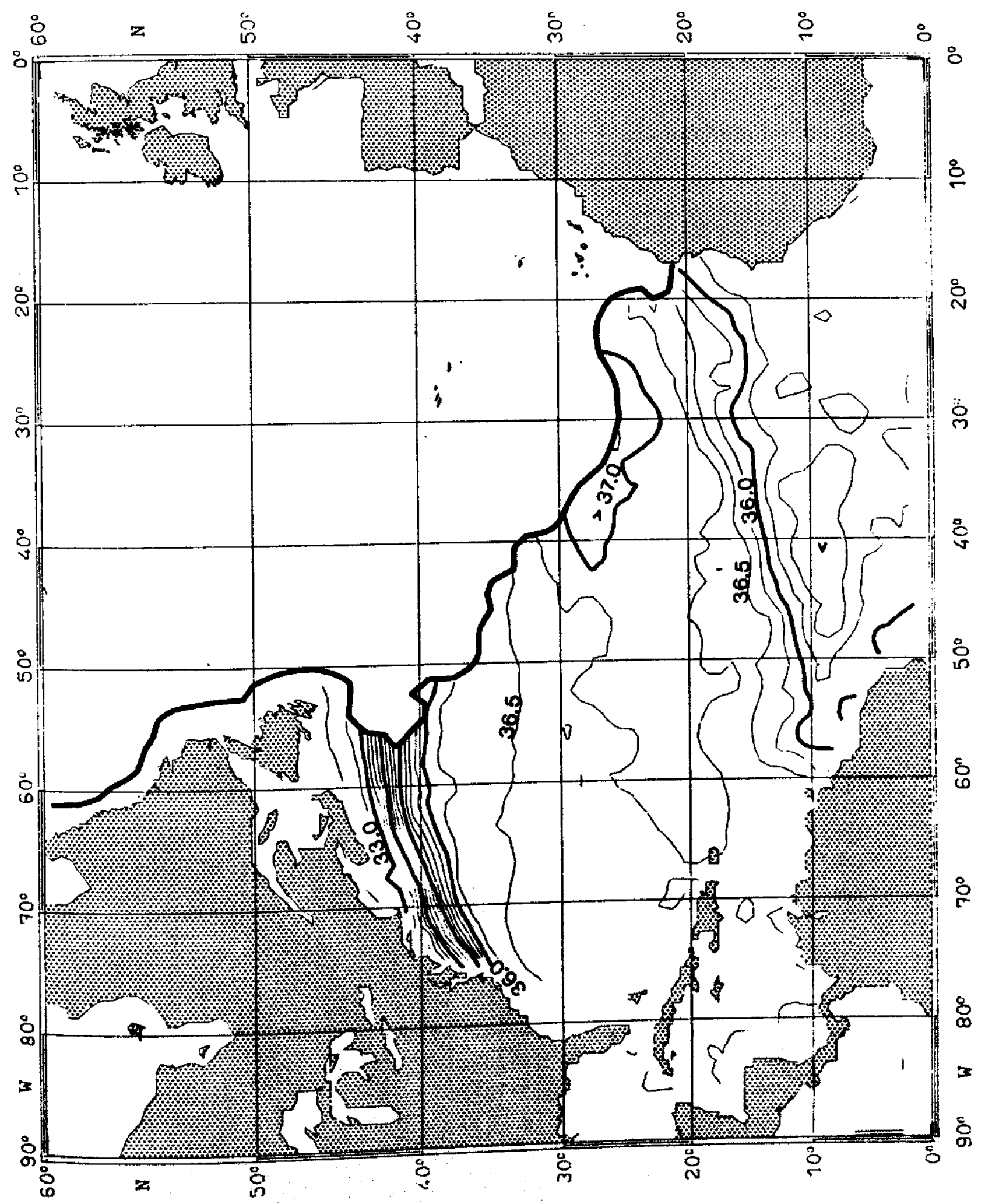


Fig. 52:

PRESSURE $\left(10^{4} \mathrm{~Pa}\right)$ on $\sigma_{\theta}=27.0 \mathrm{~kg} \mathrm{~m}^{-3}$

MAY

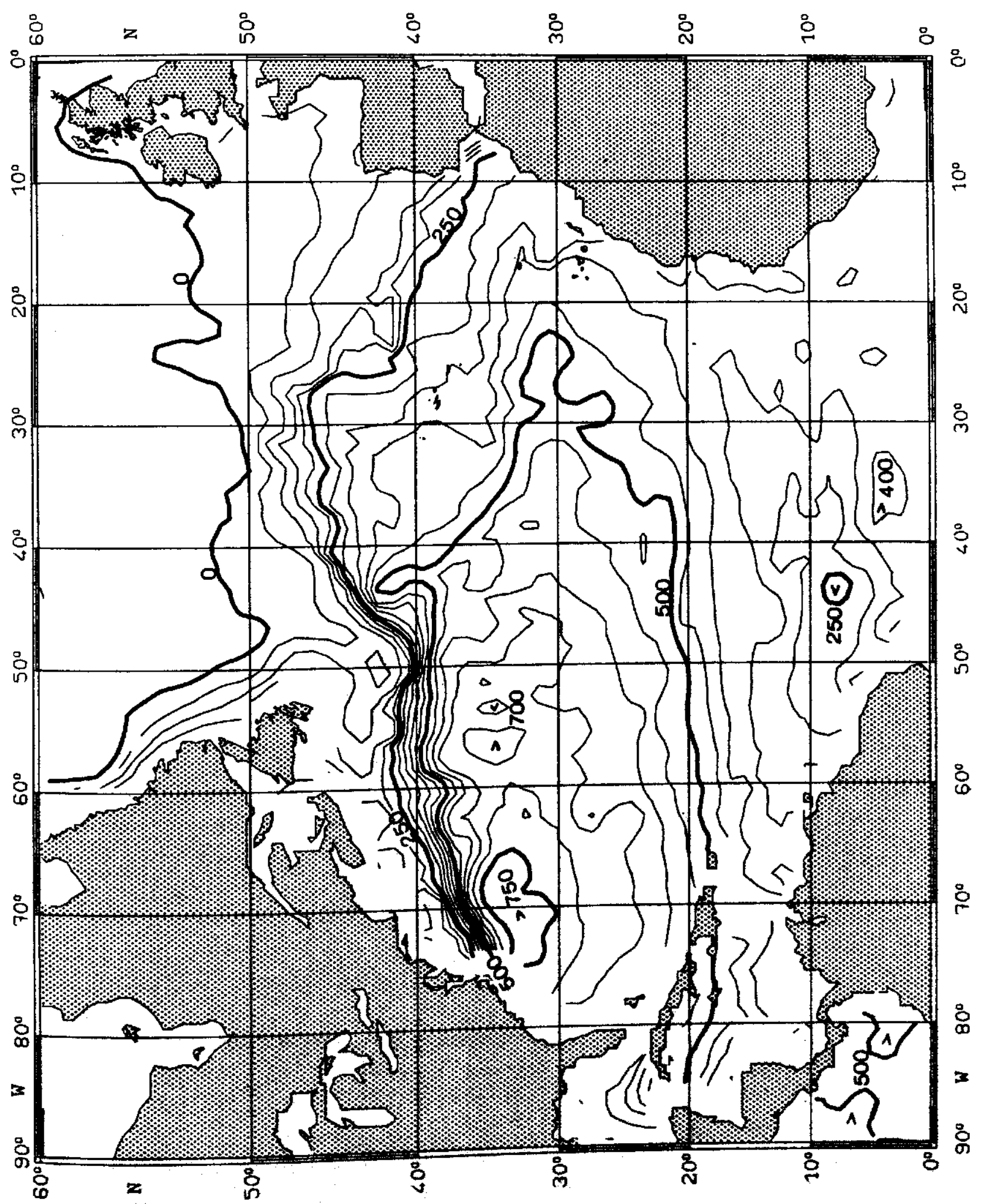




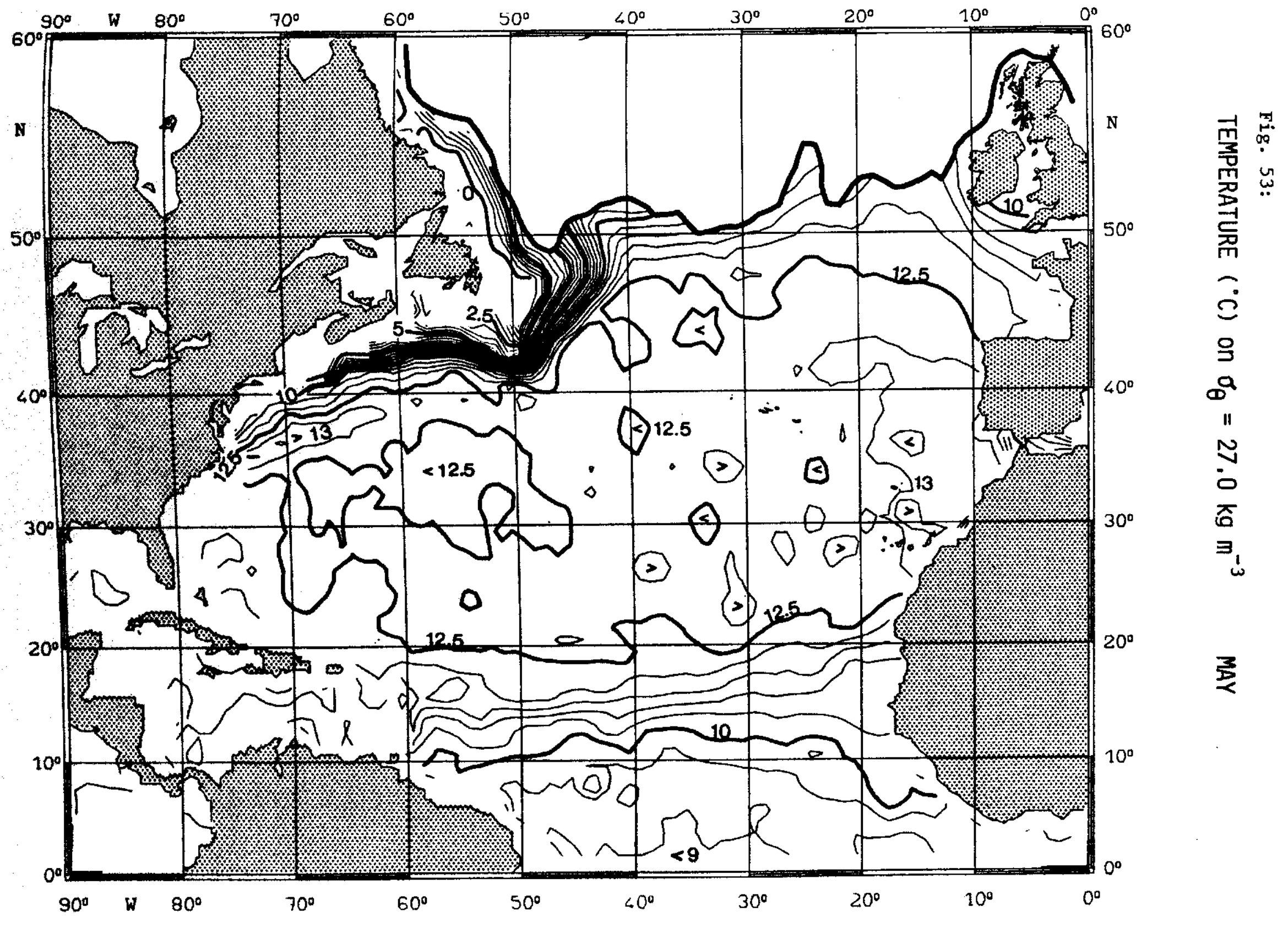


Fig. 54:

SALINITY $\left(10^{-3}\right)$ on $\sigma_{\theta}=27.0 \mathrm{~kg} \mathrm{~m}^{-3} \quad$ MAY

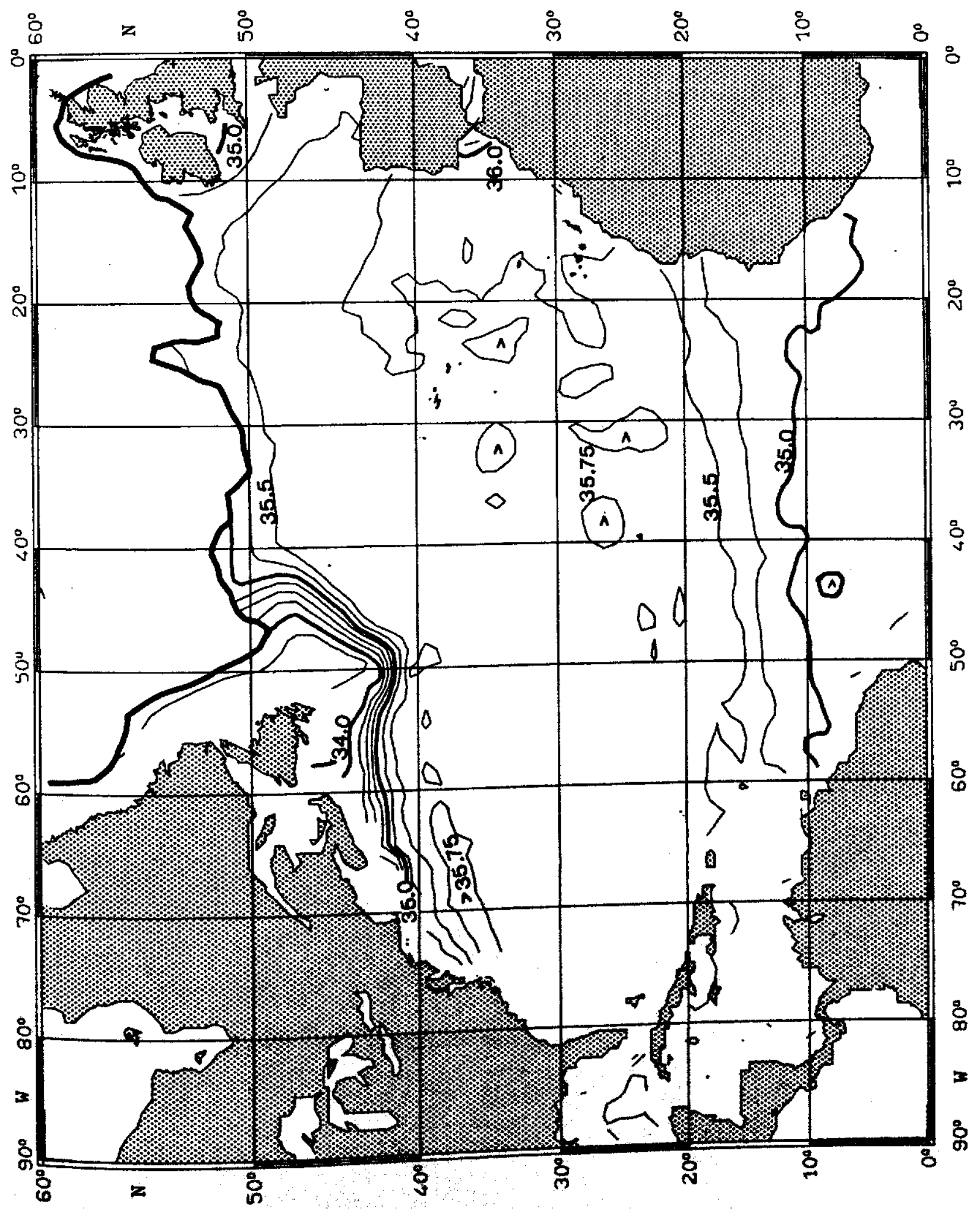


Fig. 55:

PRESSURE $\left(10^{4} \mathrm{~Pa}\right)$ on $\sigma_{\theta}=25.0 \mathrm{~kg} \mathrm{~m}^{-3}$ JUNE

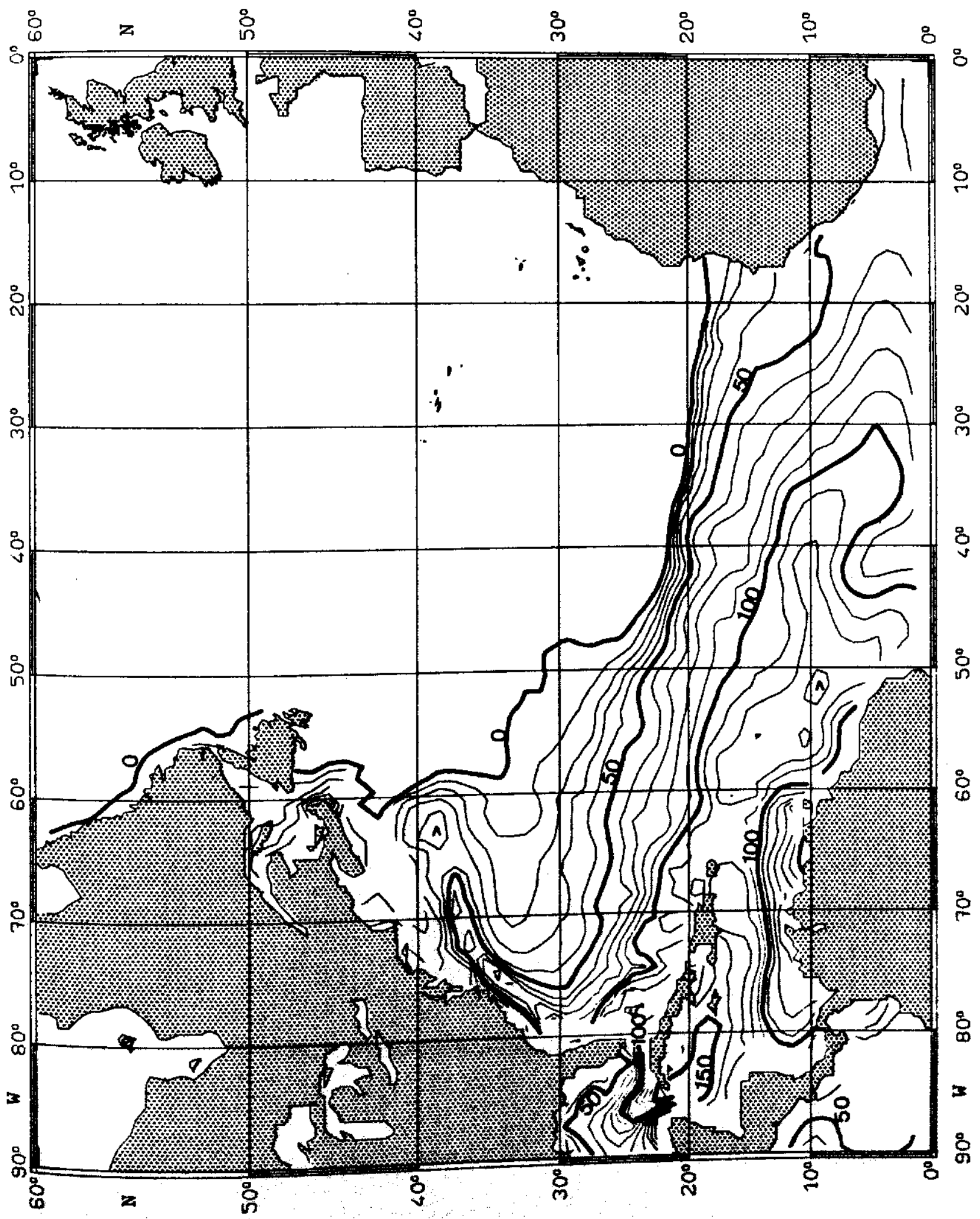


Fig. 57:

TEMPERATURE $\left({ }^{\circ} \mathrm{C}\right)$ on $\sigma_{\theta}=25.0 \mathrm{~kg} \mathrm{~m}^{-3}$ JUNE

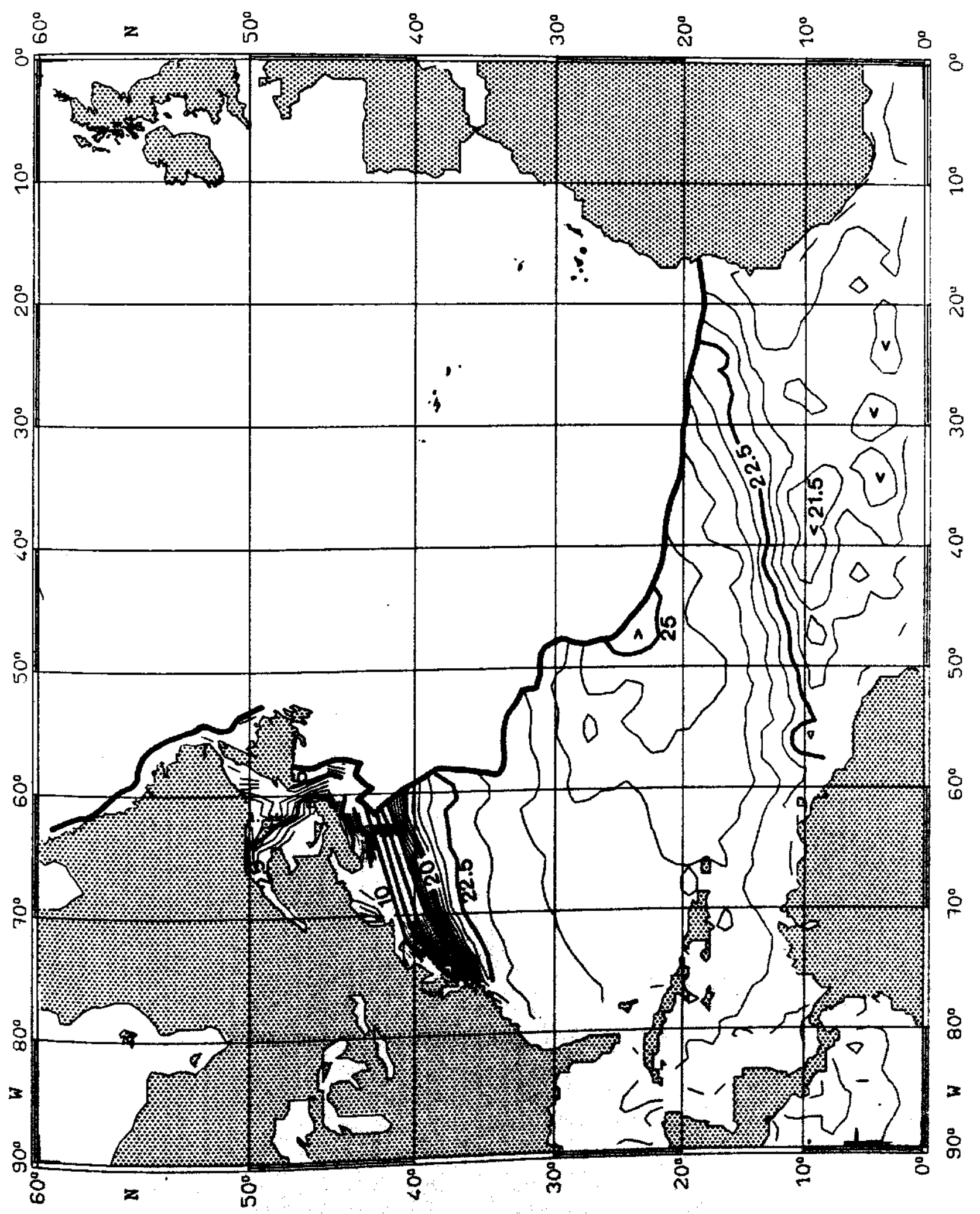


Fig. 58:

SALINITY $\left(10^{-3}\right)$ on $\sigma_{\theta}=25.0 \mathrm{~kg} \mathrm{~m}^{-3} \quad$ JUNE

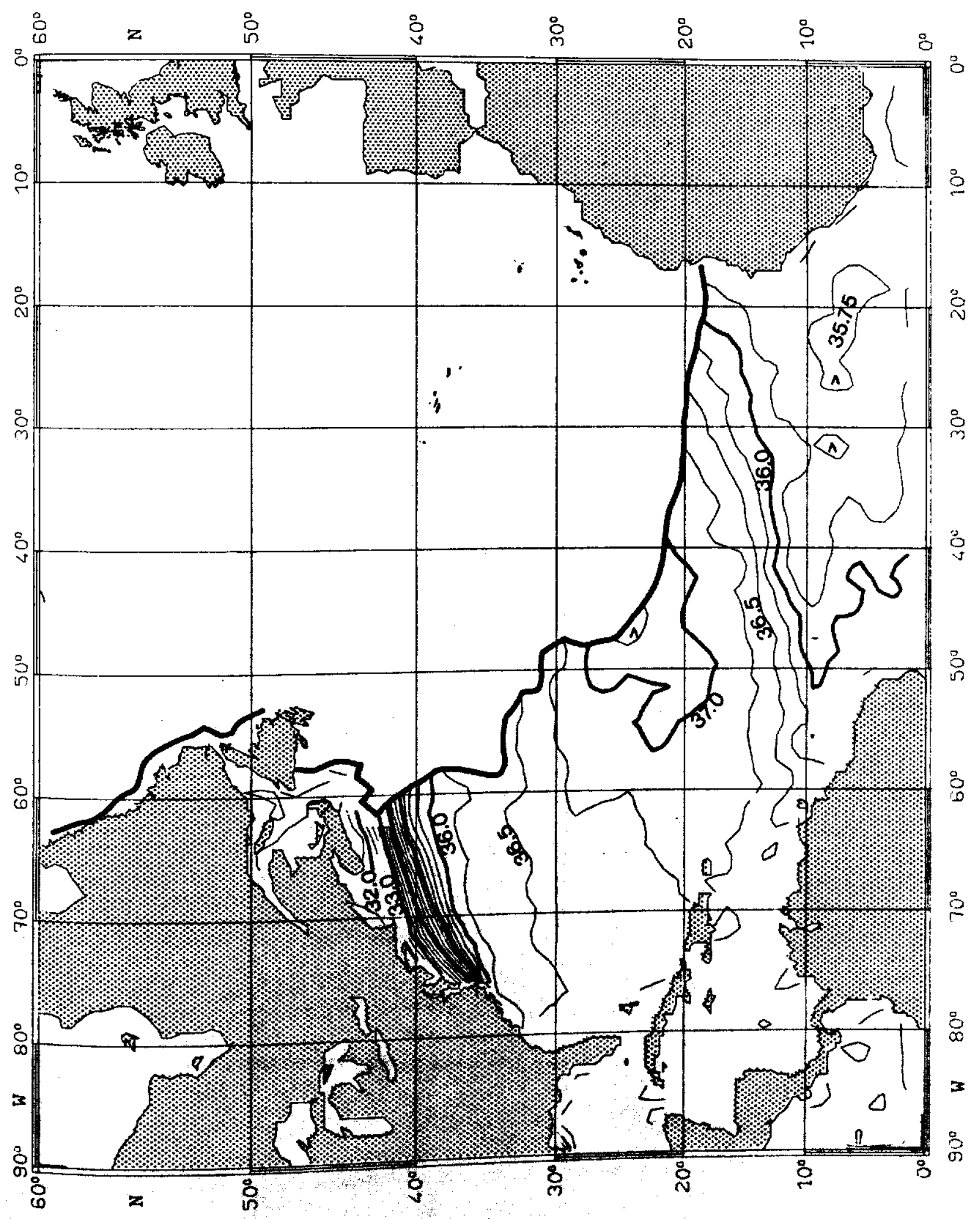


Fig. 58:

PRESSURE $\left(10^{4} \mathrm{~Pa}\right)$ on $\sigma_{\theta}=25.5 \mathrm{~kg} \mathrm{~m}^{-3}$ JUNE

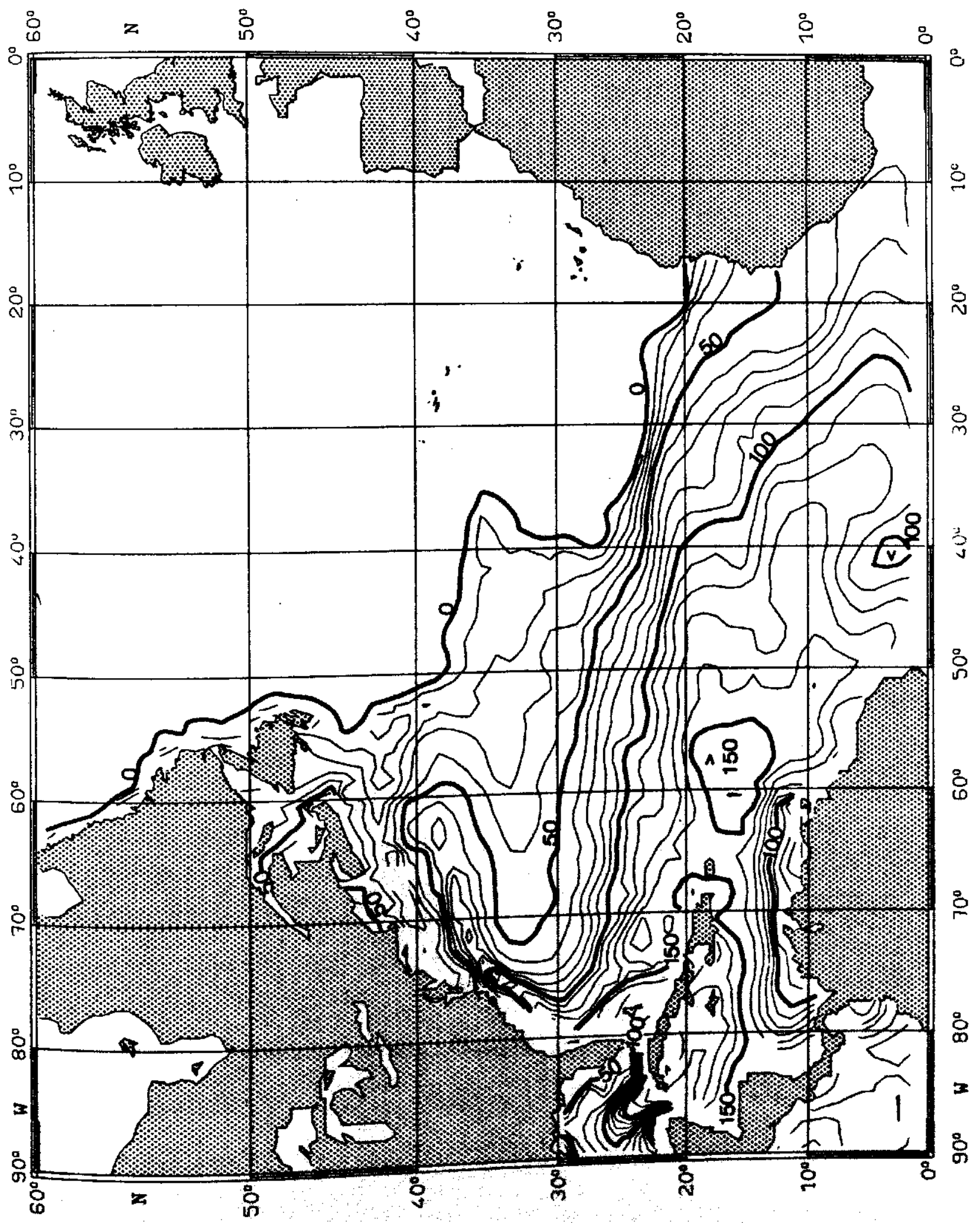


Fig. 59:

TEMPERATURE $\left({ }^{\circ} \mathrm{C}\right)$ on $\sigma_{\theta}=25.5 \mathrm{~kg} \mathrm{~m}^{-3}$ JUNE

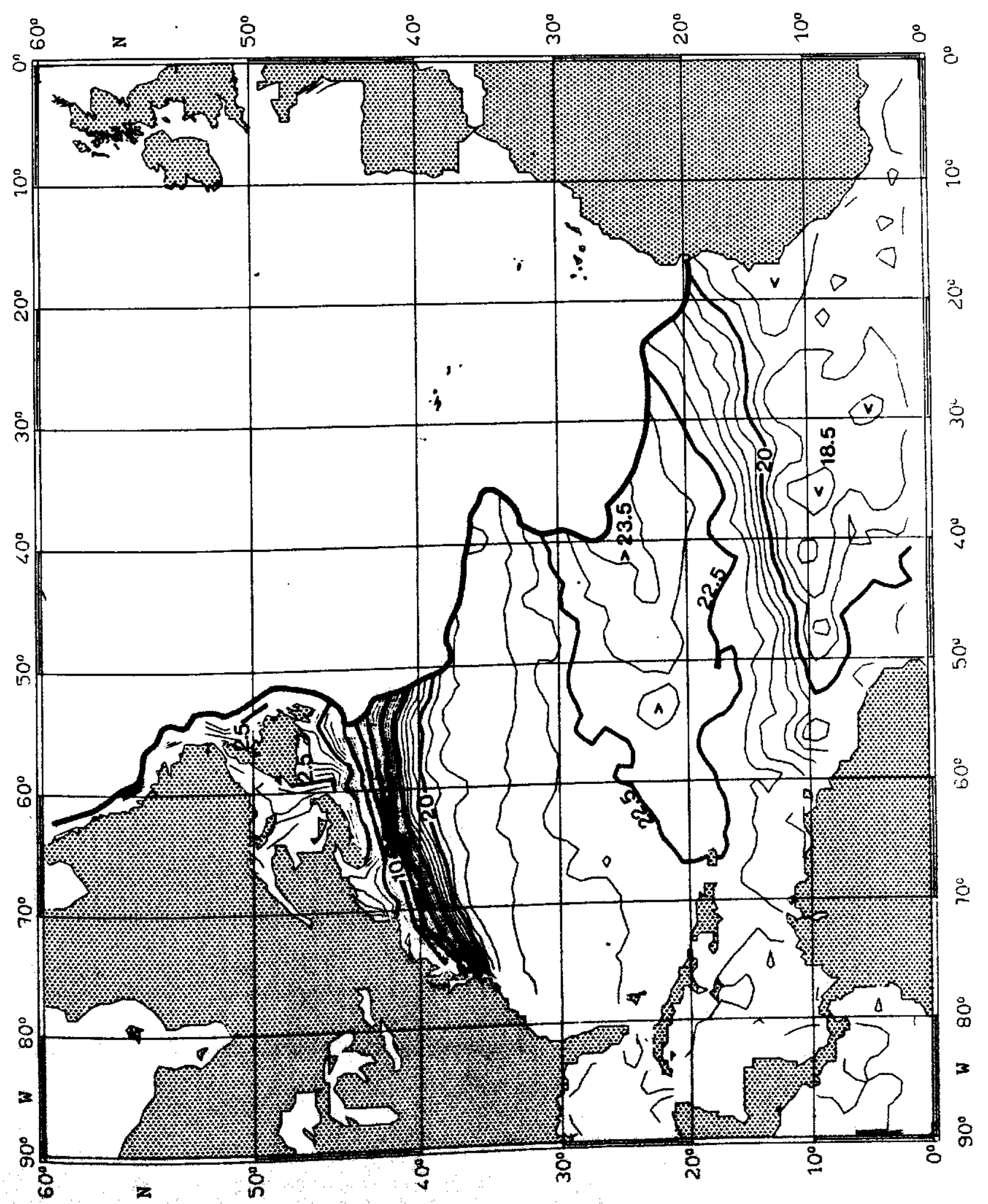


Fig. 60:

SALINITY $\left(10^{-3}\right)$ on $\sigma_{\theta}=25.5 \mathrm{~kg} \mathrm{~m}^{-3} \quad$ JUNE

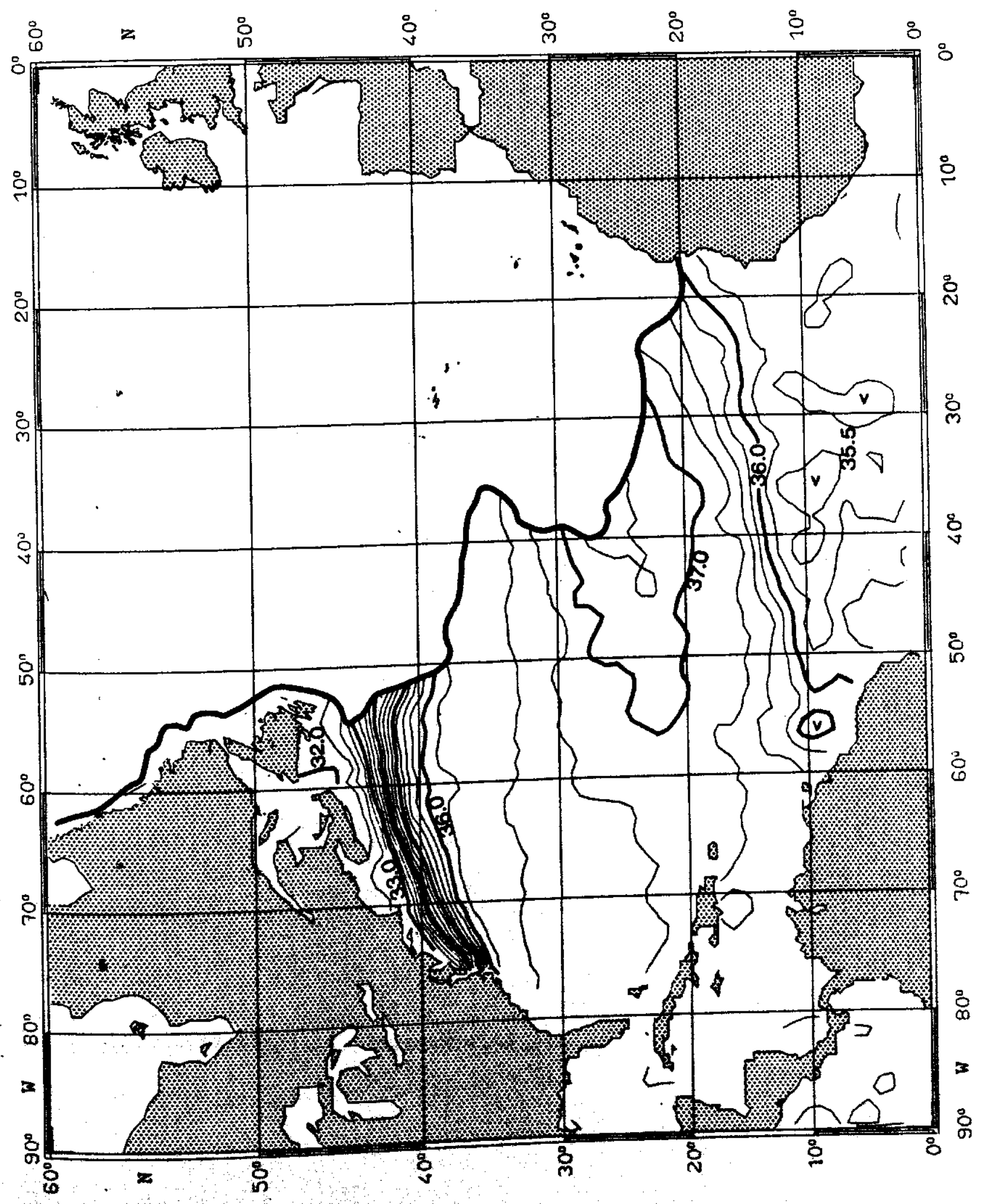


Fig. 61:

PRESSURE $\left(10^{4} \mathrm{~Pa}\right)$ on $\sigma_{\theta}=26.0 \mathrm{~kg} \mathrm{~m}^{-3}$ JUNE

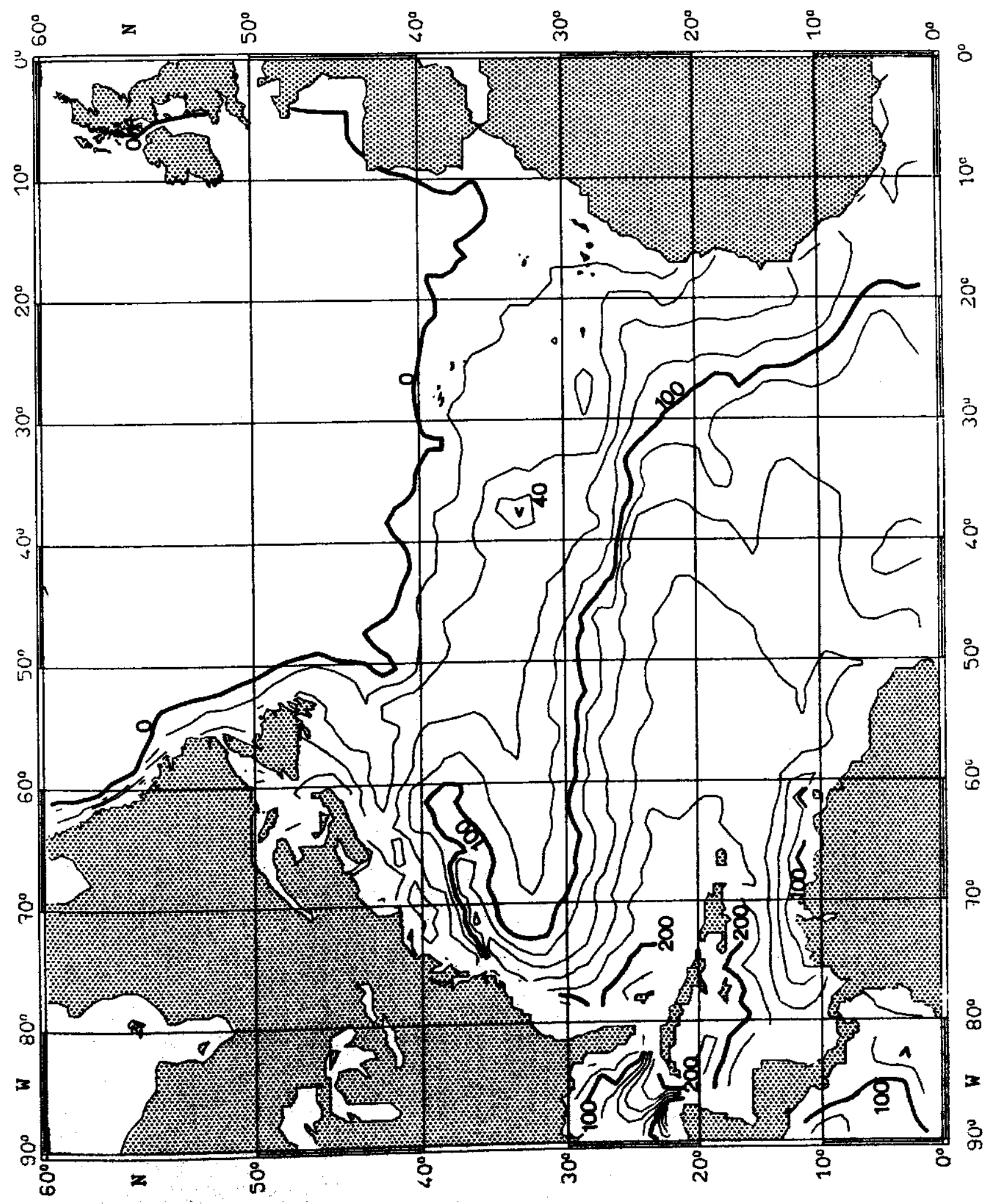


Fig. 62:

TEMPERATURE $\left({ }^{\circ} \mathrm{C}\right)$ on $\sigma_{\theta}=26.0 \mathrm{~kg} \mathrm{~m}^{-3}$ JUNE

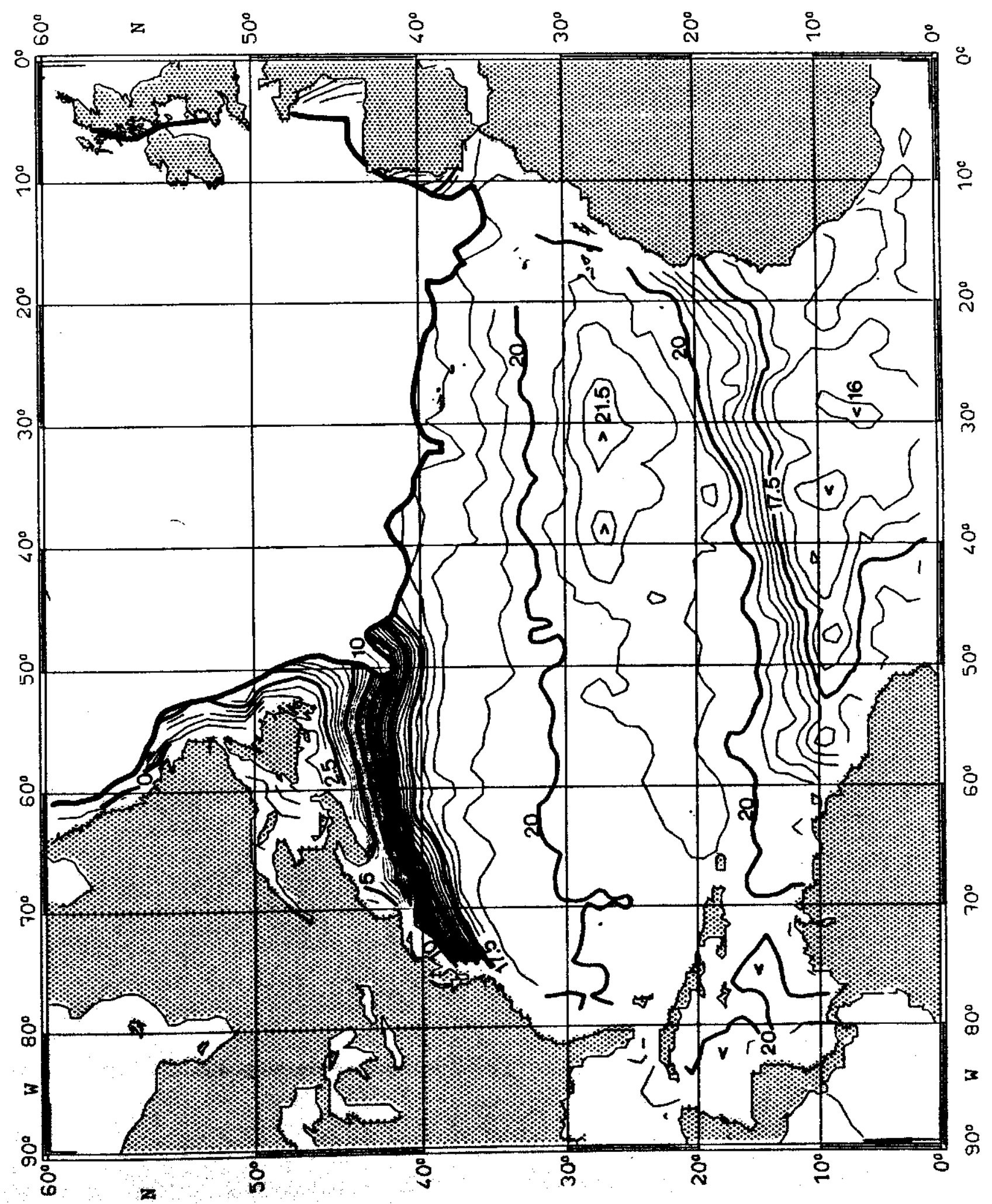


Fig. 63:

SALINITY $\left(10^{-3}\right)$ on $\sigma_{\theta}=26.0 \mathrm{~kg} \mathrm{~m}^{-3} \quad$ JUNE

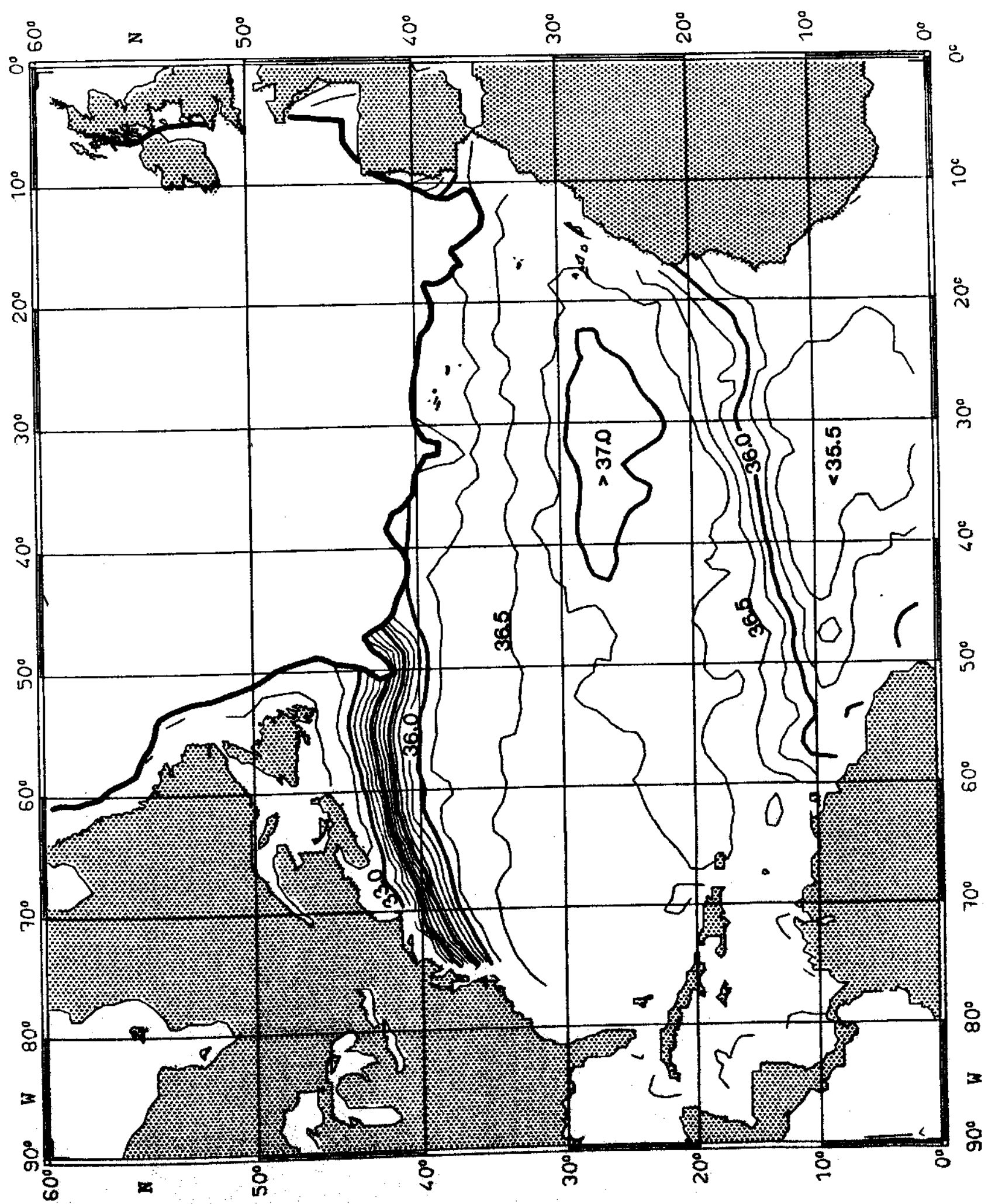


Fig. 64:

PRESSURE $\left(10^{4} \mathrm{~Pa}\right)$ on $\sigma_{\theta}=26.5 \mathrm{~kg} \mathrm{~m}^{-3}$ JUNE

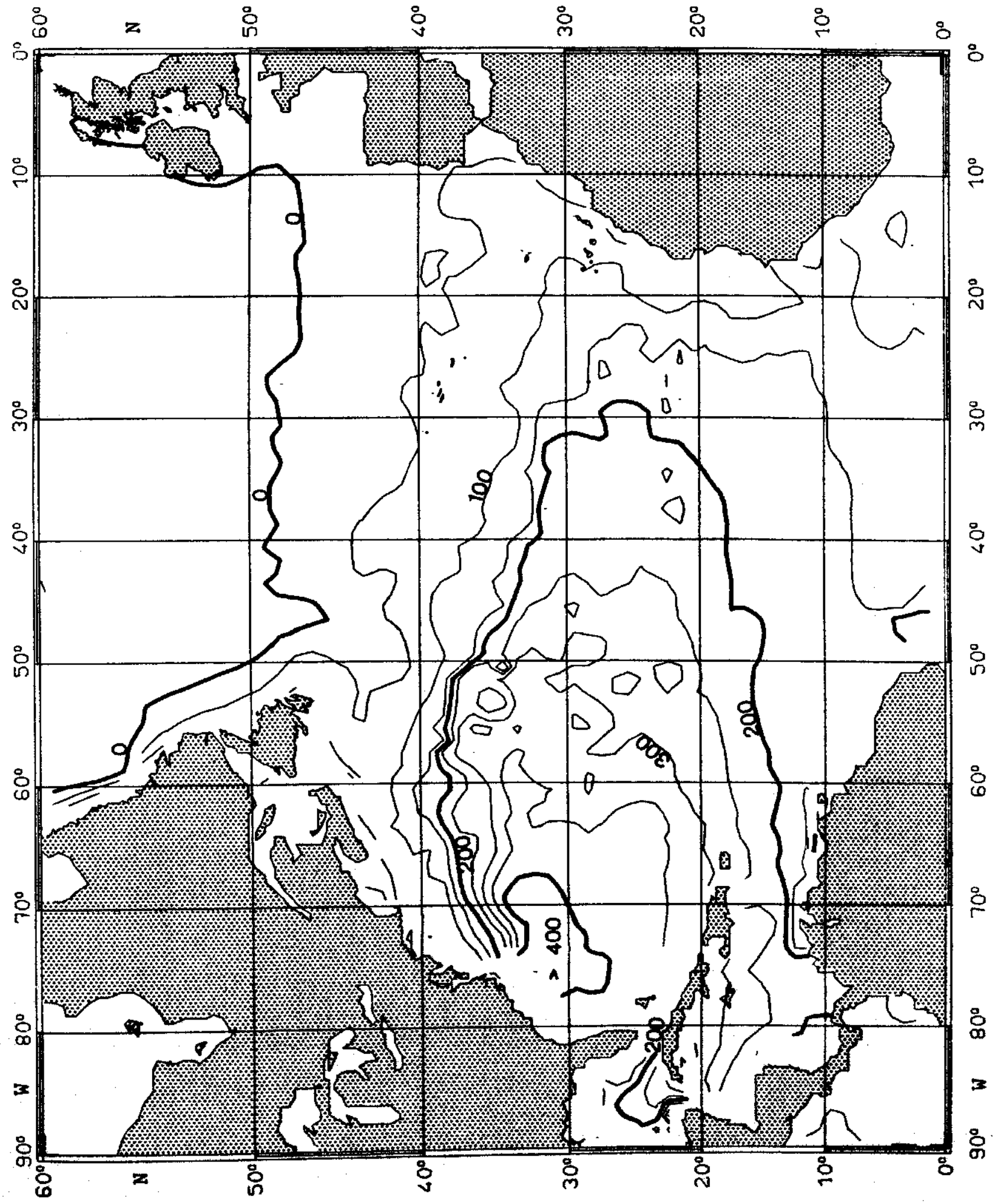


Fig. 65:

TEMPERATURE $\left({ }^{\circ} \mathrm{C}\right)$ on $\sigma_{\theta}=26.5 \mathrm{~kg} \mathrm{~m}^{-3}$ JUNE

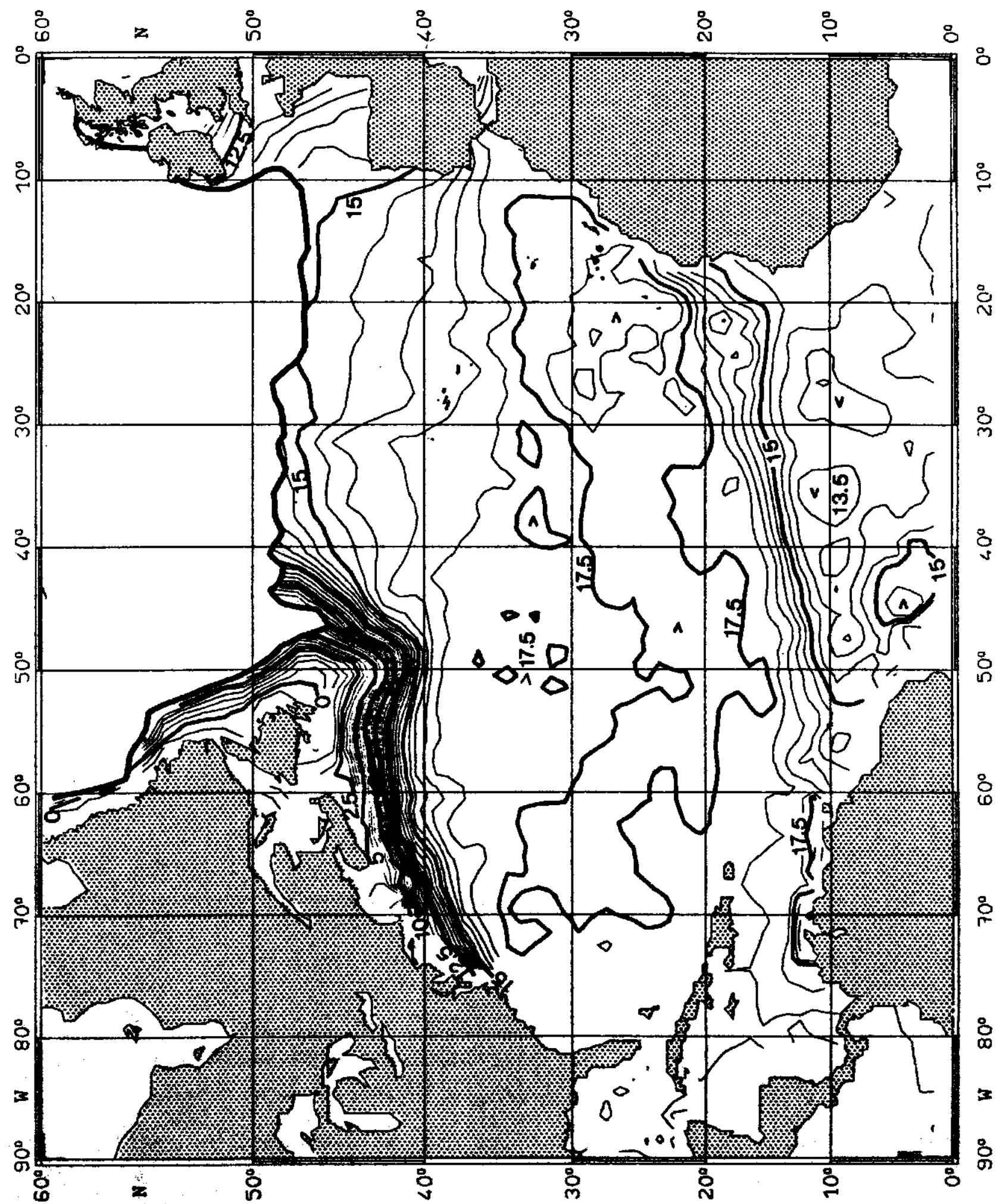


Fig. 66:

SALINITY $\left(10^{-3}\right)$ on $\sigma_{\theta}=26.5 \mathrm{~kg} \mathrm{~m}^{-3} \quad$ JUNE

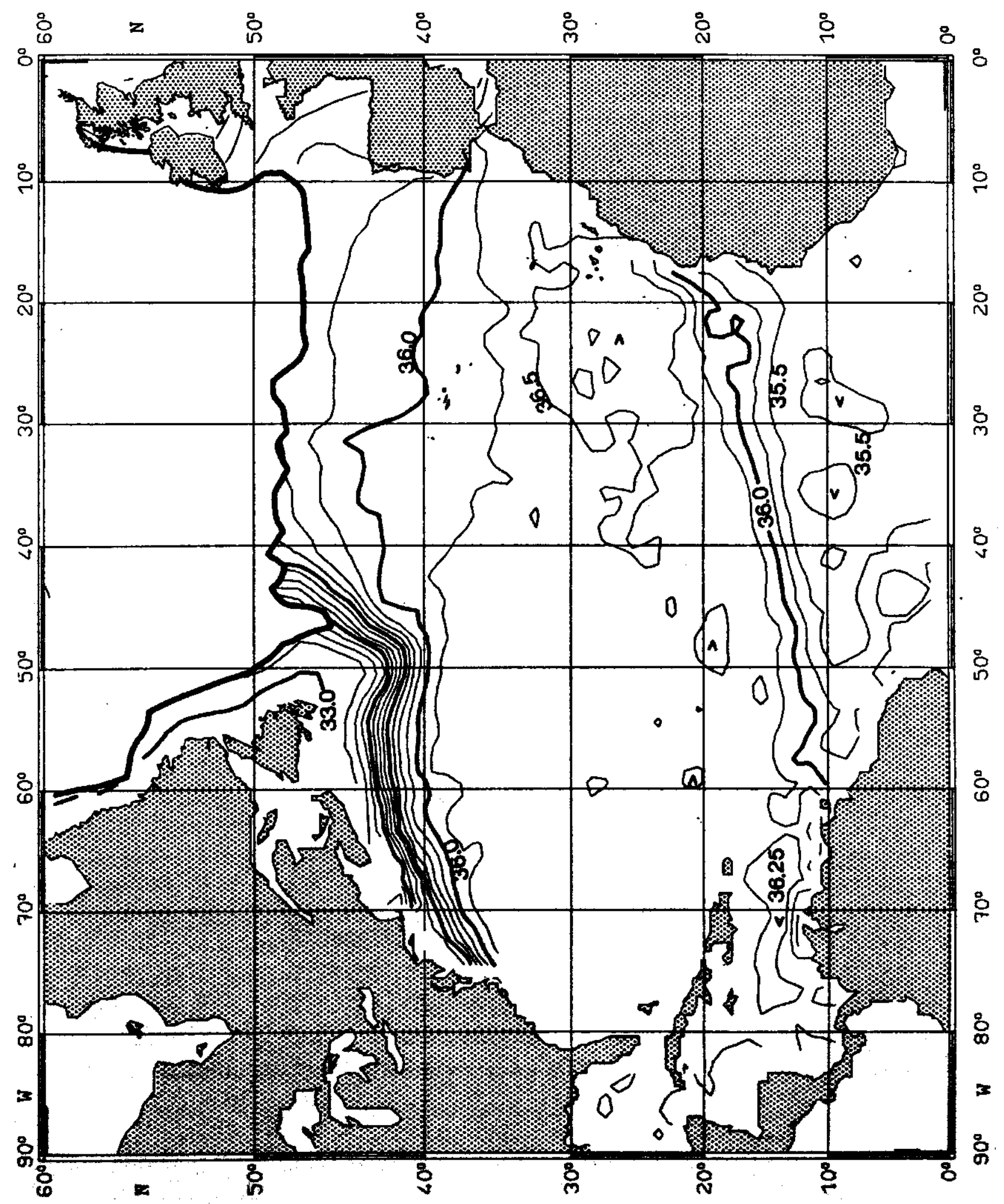


Fig. 67:

PRESSURE $\left(10^{4} \mathrm{~Pa}\right)$ on $\sigma_{\theta}=27.0 \mathrm{~kg} \mathrm{~m}^{-3}$ JUNE

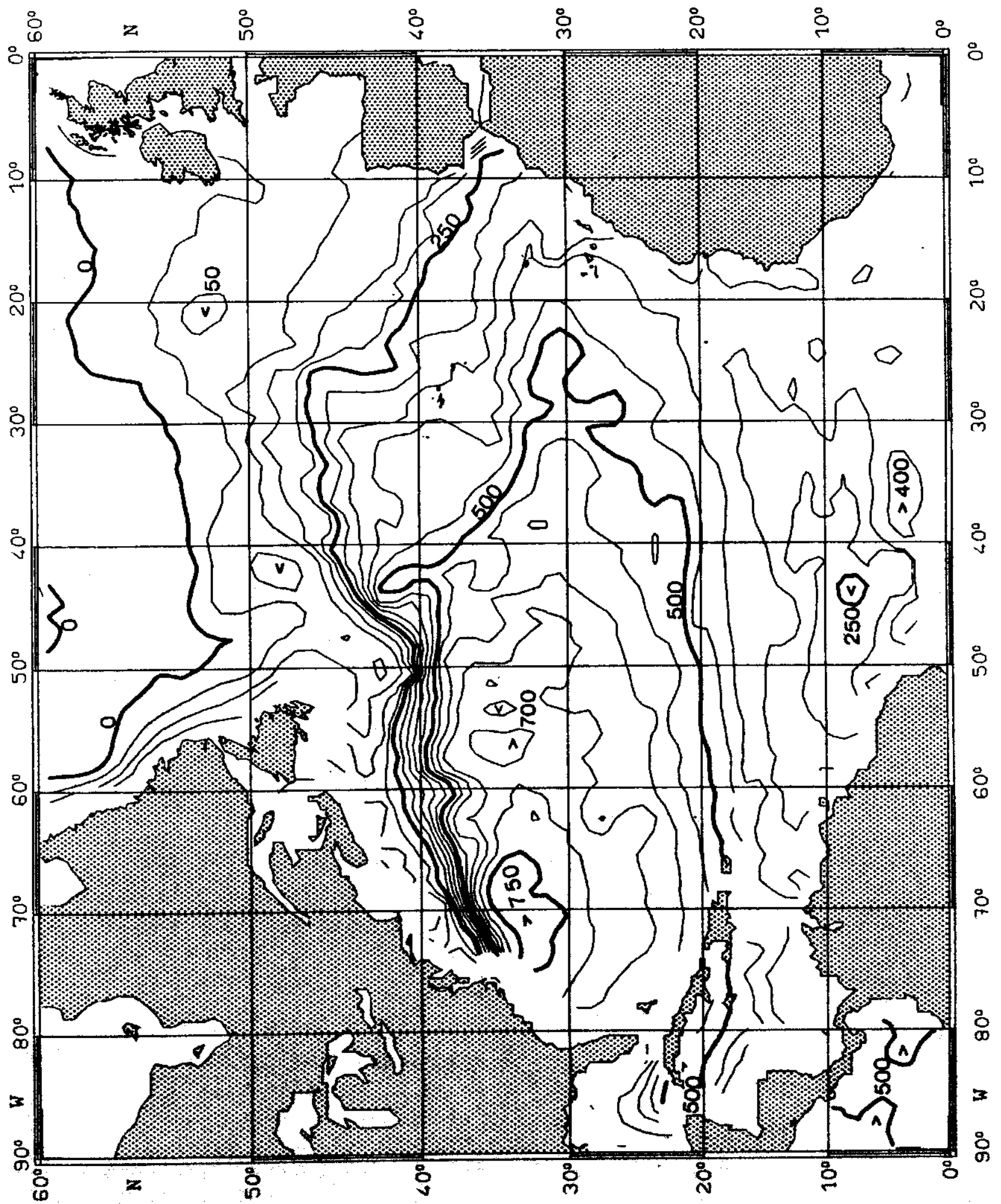


Fig. 68:

TEMPERATURE $\left({ }^{\circ} \mathrm{C}\right)$ on $\sigma_{\theta}=27.0 \mathrm{~kg} \mathrm{~m}^{-3}$ JUNE

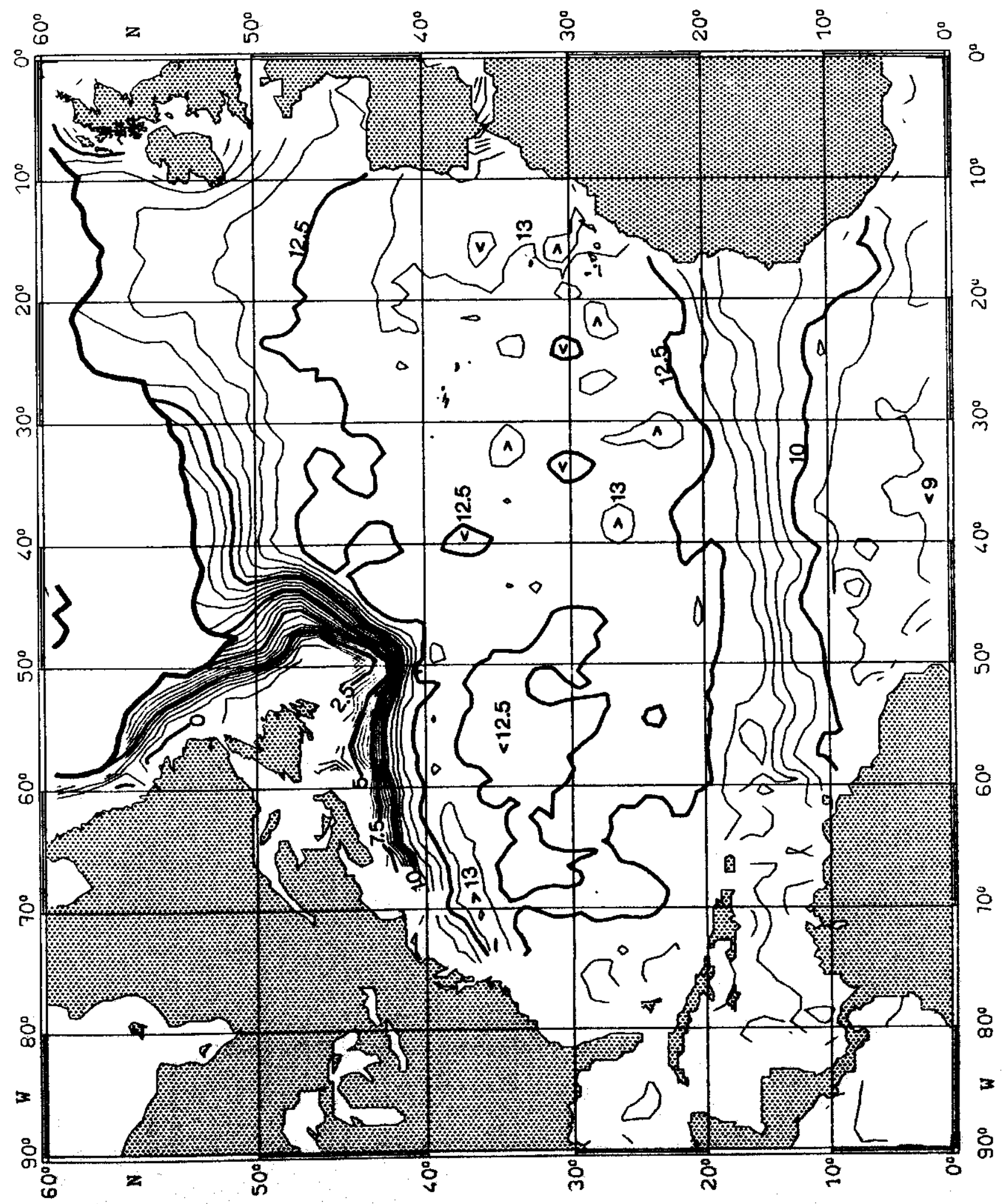


Fig. 69:

SALINITY $\left(10^{-3}\right)$ on $\sigma_{\theta}=27.0 \mathrm{~kg} \mathrm{~m}^{-3}$ JUNE

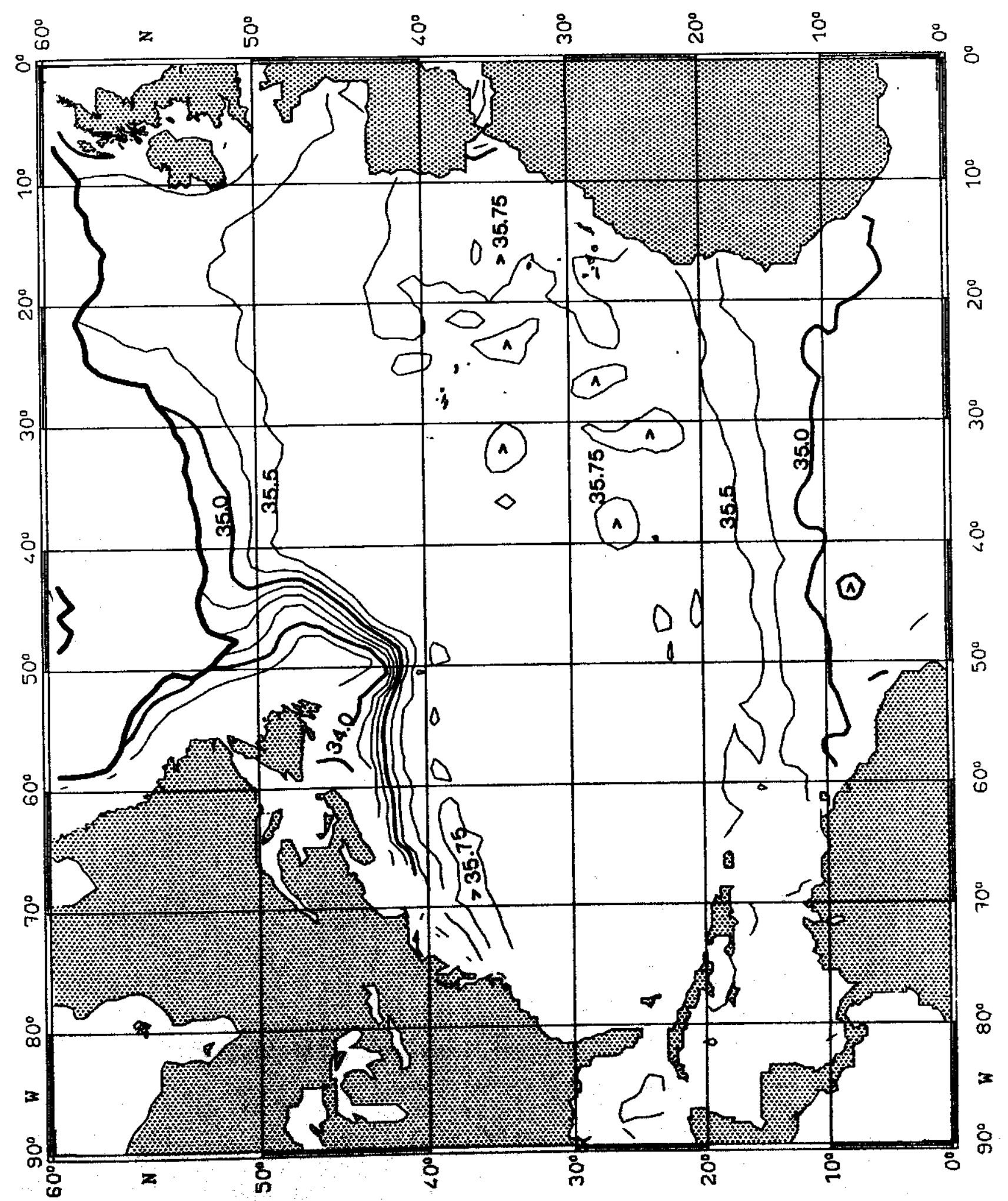


Fig. 70:

PRESSURE $\left(10^{4} \mathrm{~Pa}\right)$ on $\sigma_{\theta}=25.0 \mathrm{~kg} \mathrm{~m}^{-3} \quad$ JULY

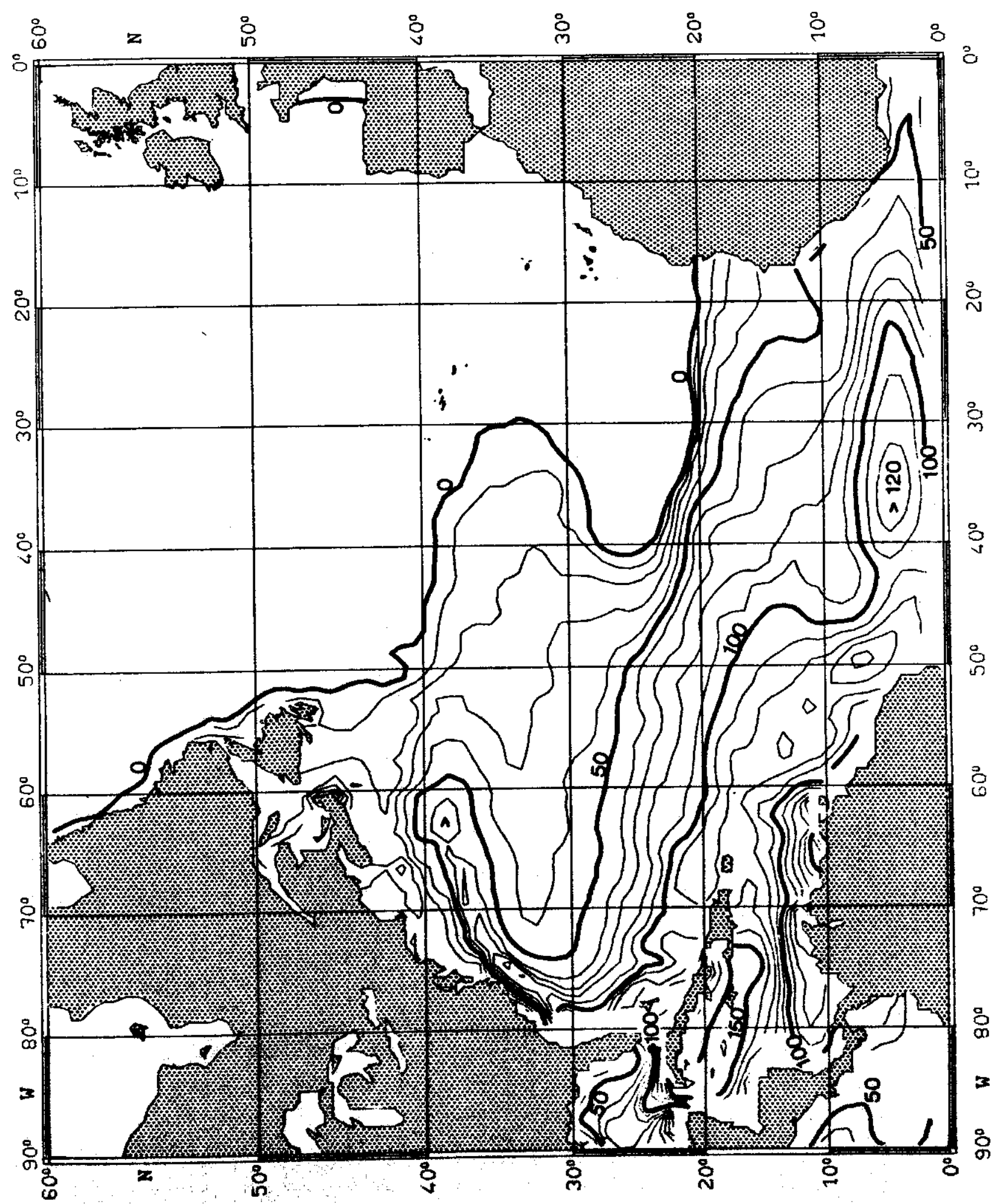


Fig. 71:

TEMPERATURE $\left({ }^{\circ} \mathrm{C}\right)$ on $\sigma_{\theta}=25.0 \mathrm{~kg} \mathrm{~m}^{-3} \quad$ JULY

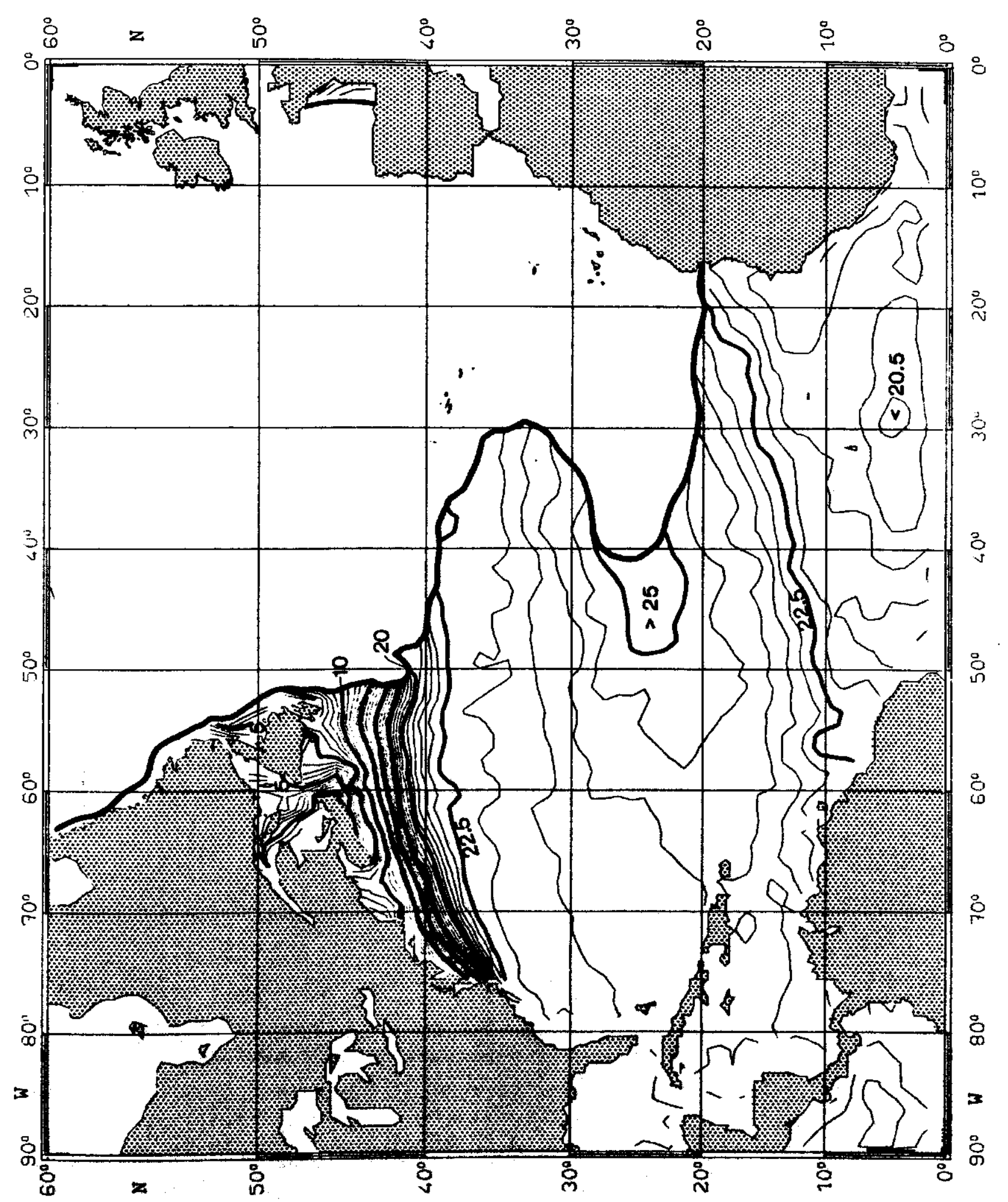


Fig. 72:

SALINITY $\left(10^{-3}\right)$ on $\sigma_{\theta}=25.0 \mathrm{~kg} \mathrm{~m}^{-3} \quad$ JULY

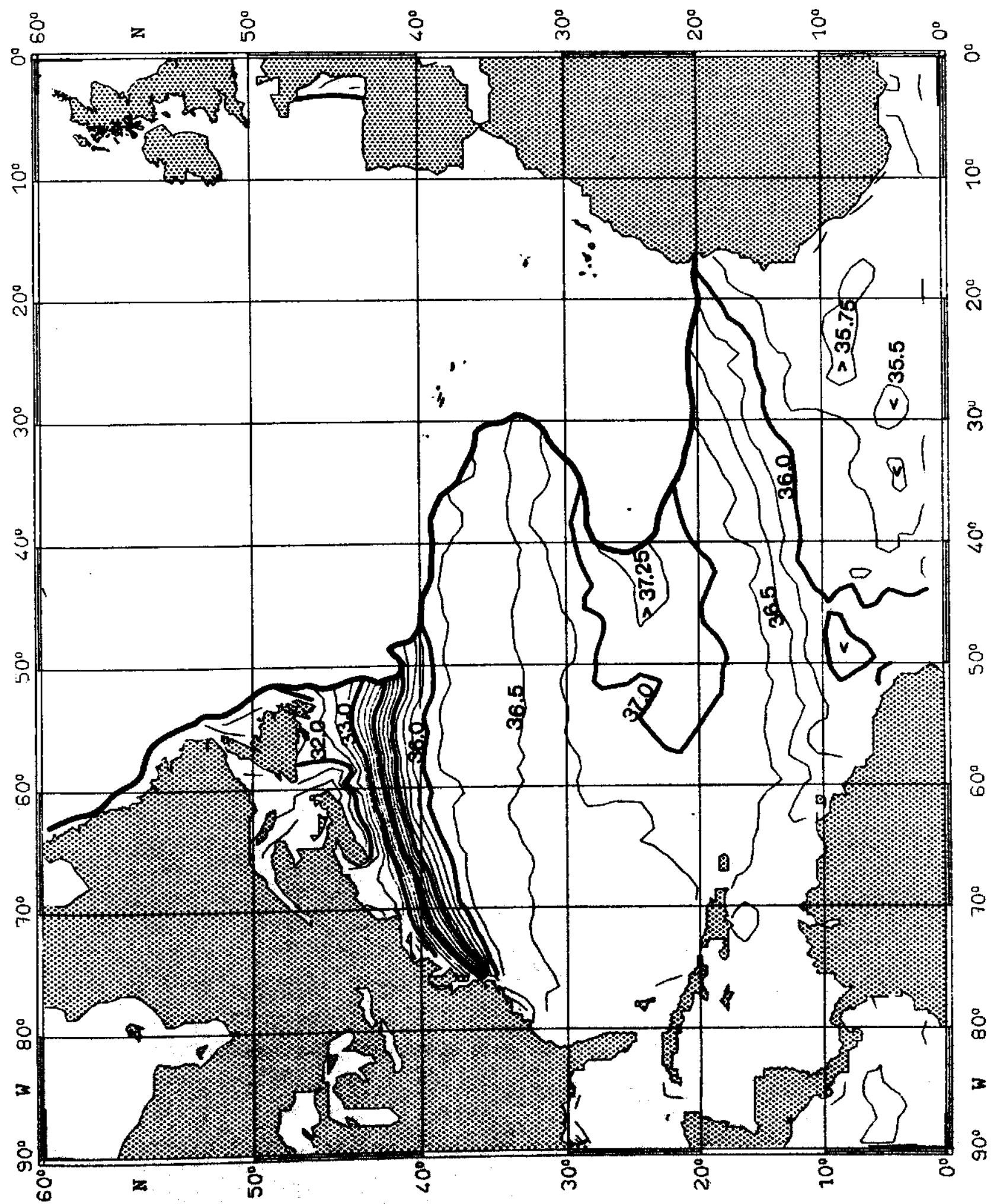


Fig. 73:

PRESSURE $\left(10^{4} \mathrm{~Pa}\right)$ on $\sigma_{\theta}=25.5 \mathrm{~kg} \mathrm{~m}^{-3} \quad$ JULY

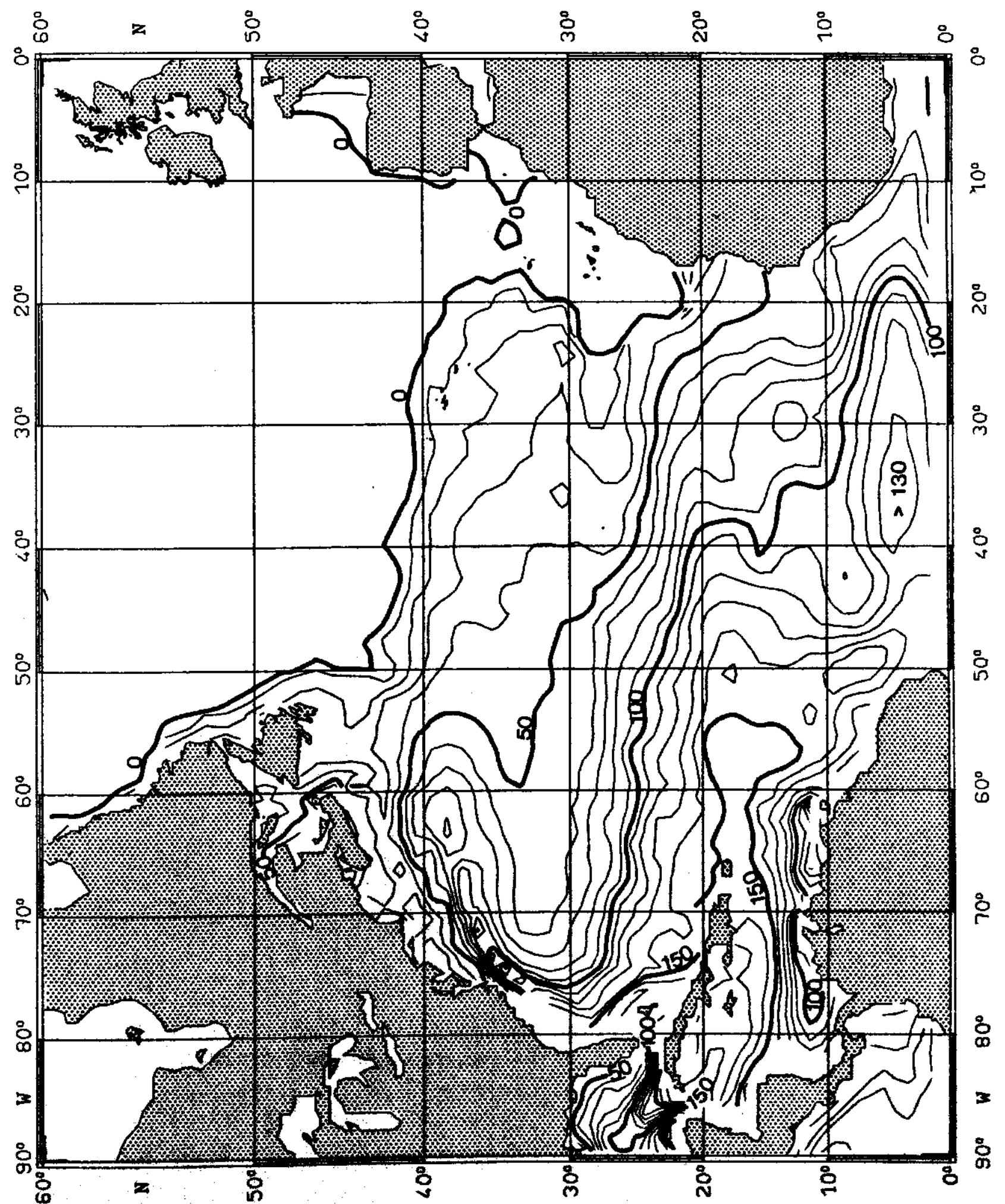


Fig. 74:

TEMPERATURE $\left({ }^{\circ} \mathrm{C}\right)$ on $\sigma_{\theta}=25.5 \mathrm{~kg} \mathrm{~m}^{-3} \quad$ JULY

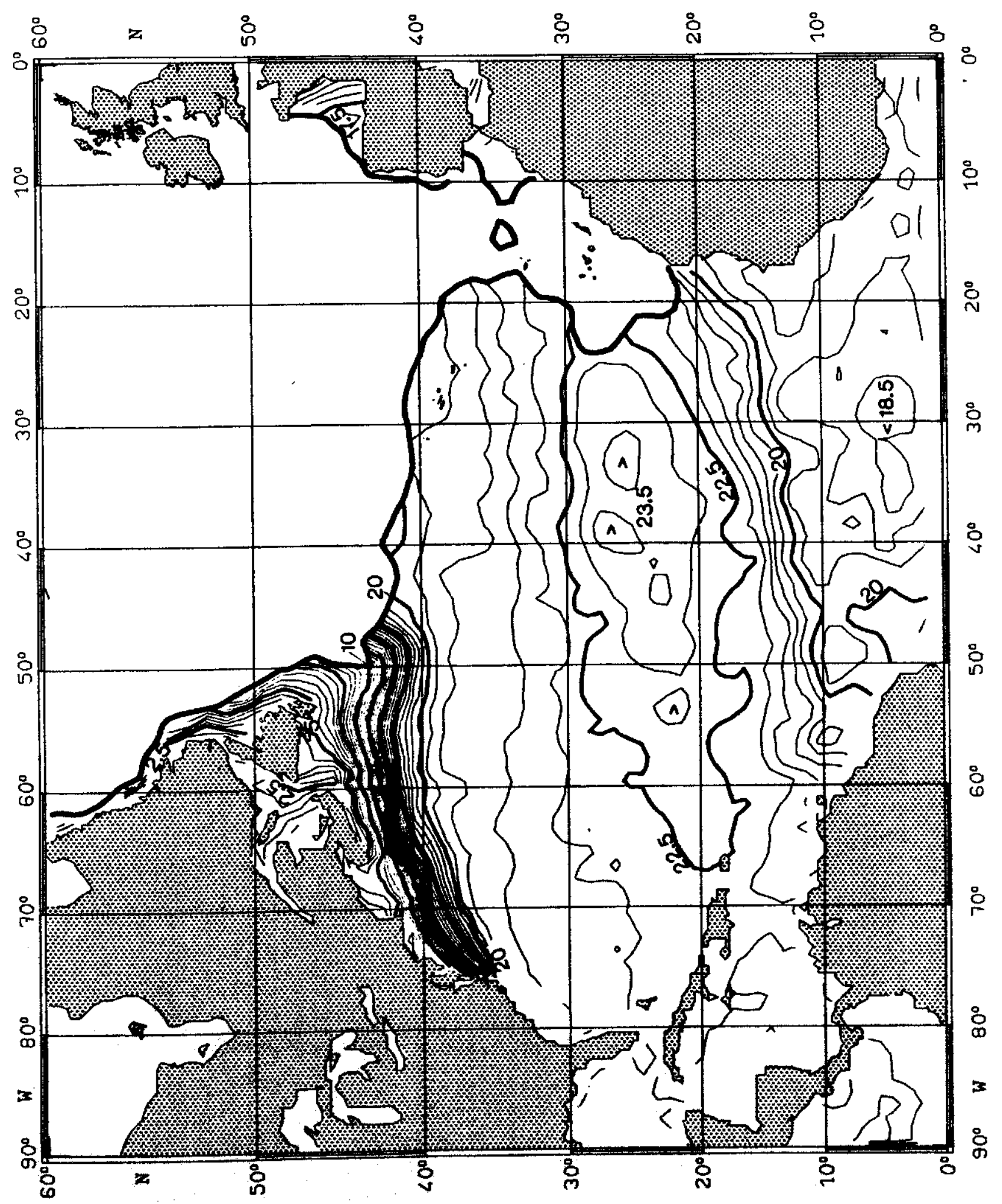


Fig. 75:

SALINITY $\left(10^{-3}\right)$ on $\sigma_{\theta}=25.5 \mathrm{~kg} \mathrm{~m}^{-3} \quad$ JULY

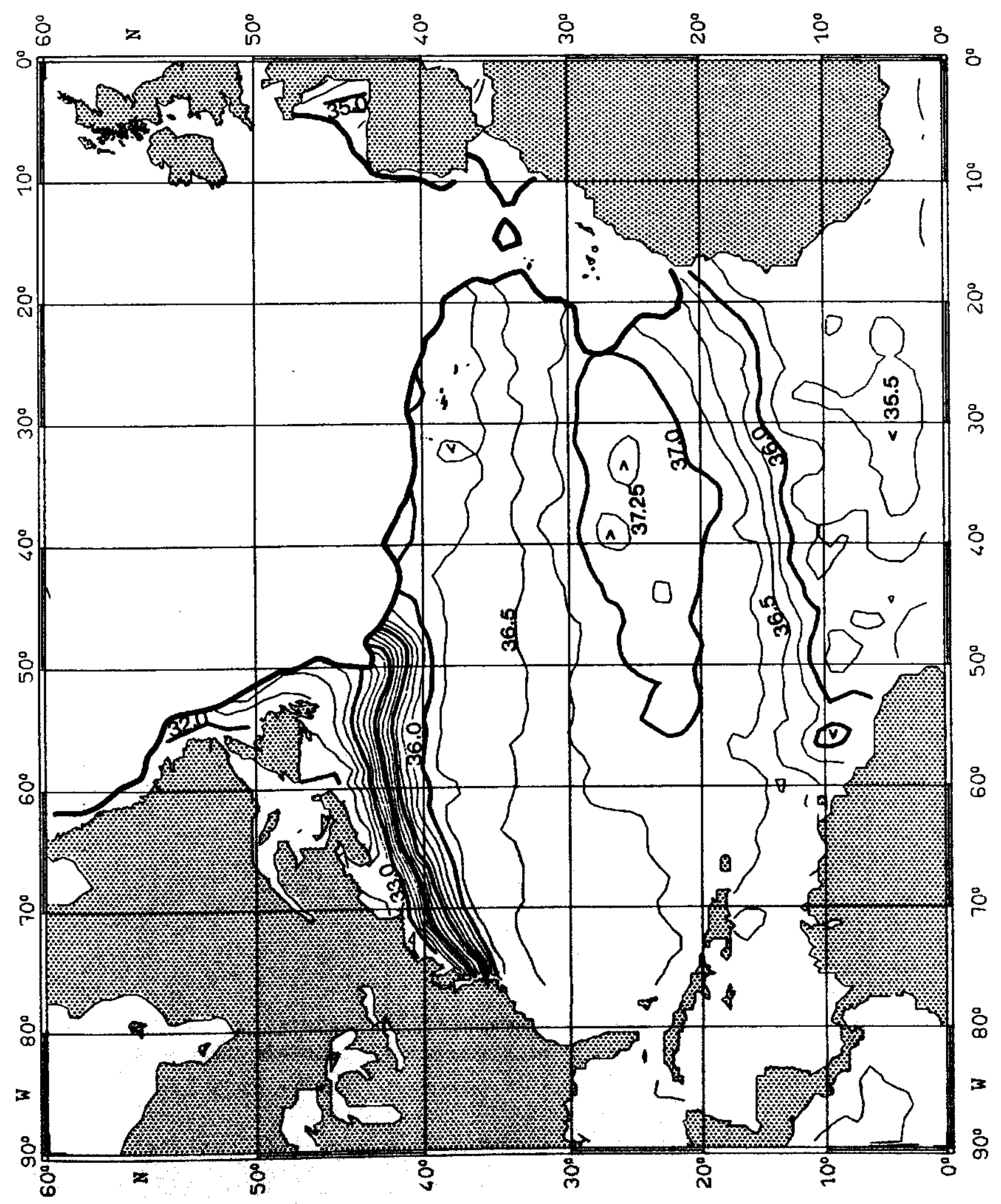


Fig. 76 :

PRESSURE $\left(10^{4} \mathrm{~Pa}\right)$ on $\sigma_{\theta}=26.0 \mathrm{~kg} \mathrm{~m}^{-3} \quad$ JULY

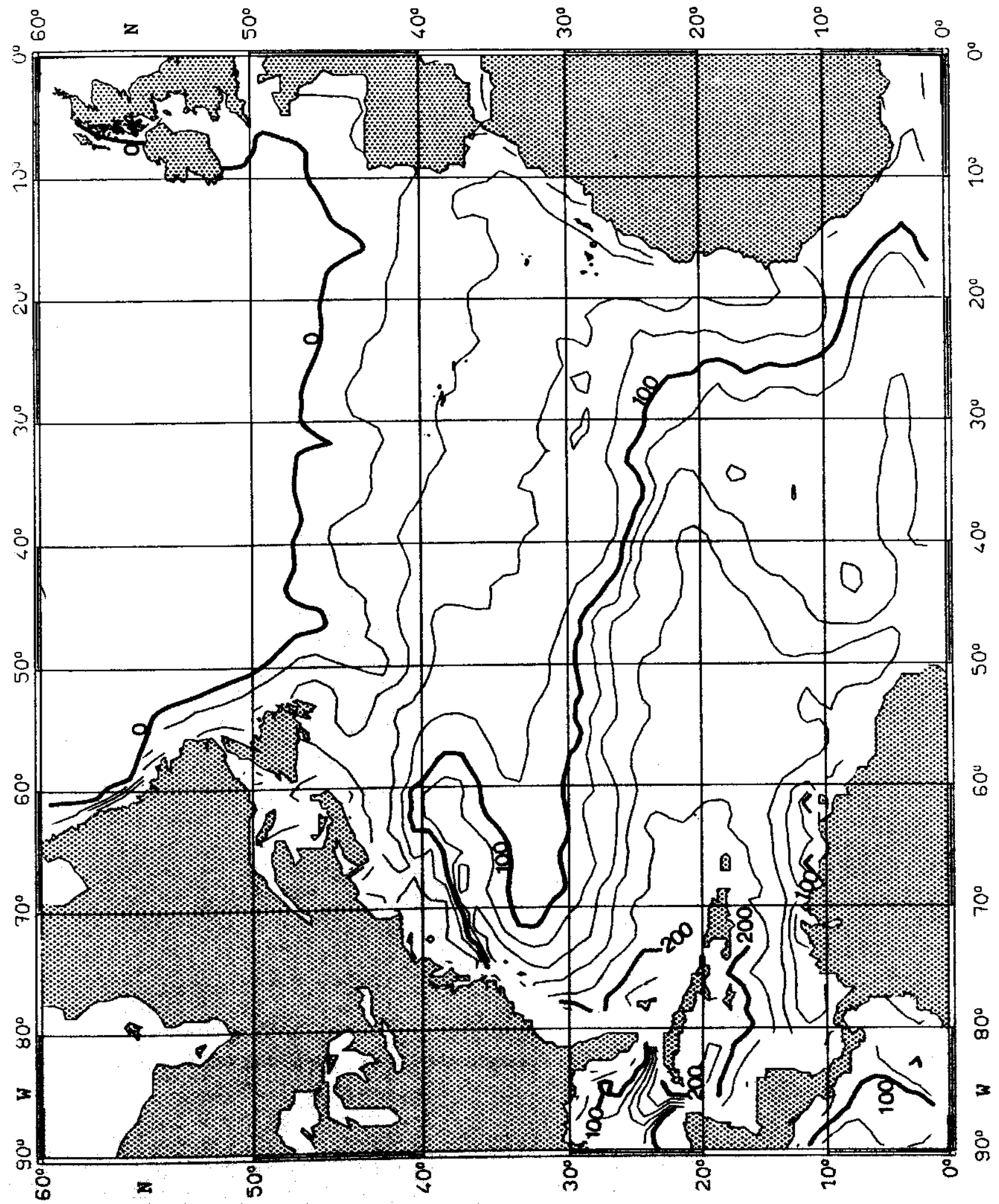


Fig. 77 :

TEMPERATURE $\left({ }^{\circ} \mathrm{C}\right)$ on $\sigma_{\theta}=26.0 \mathrm{~kg} \mathrm{~m}^{-3} \quad$ JULY

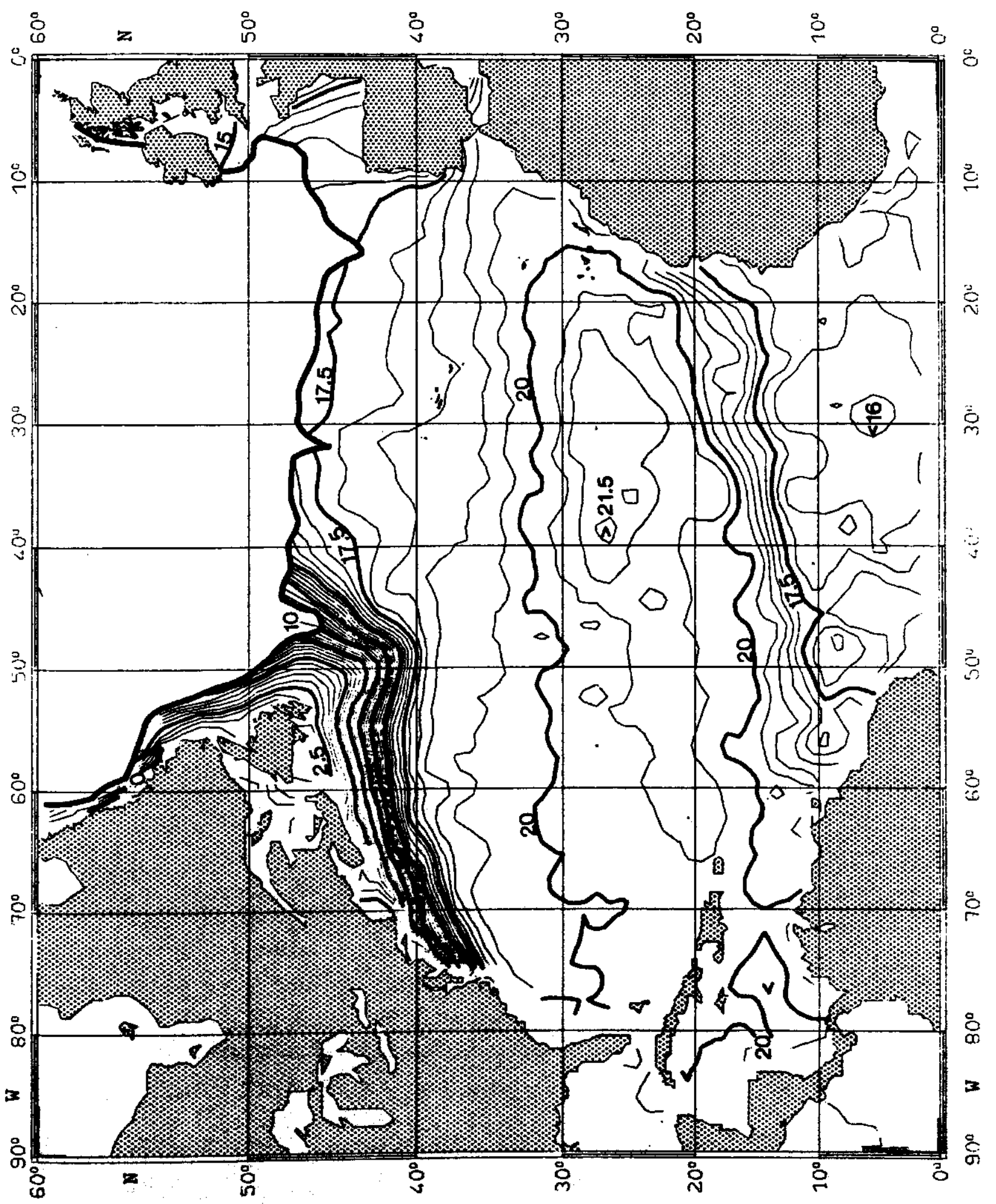


Fig. 78:

SALINITY $\left(10^{-3}\right)$ on $\sigma_{\theta}=26.0 \mathrm{~kg} \mathrm{~m}^{-3} \quad$ JULY

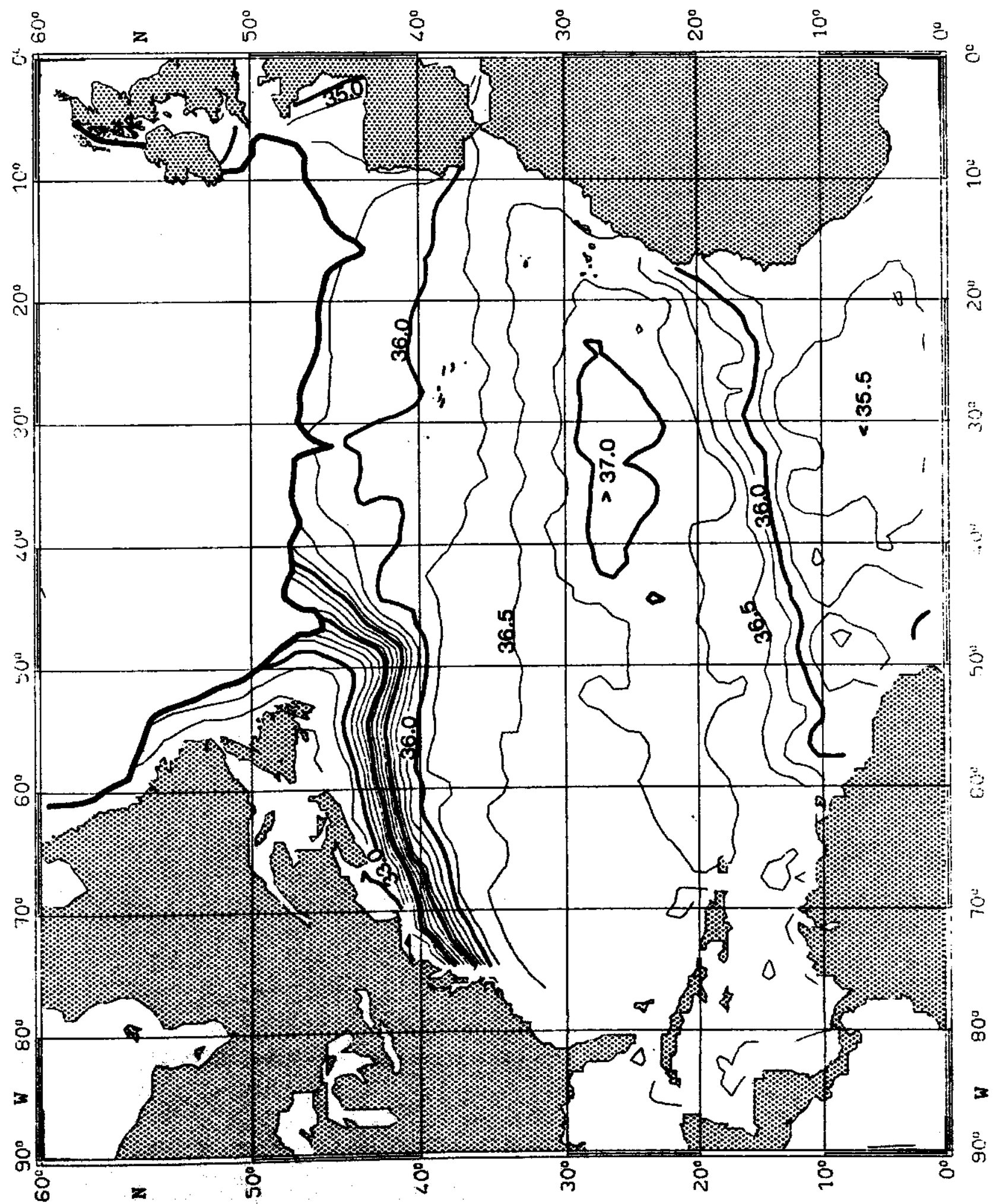


Fig. 79:

PRESSURE $\left(10^{4} \mathrm{~Pa}\right)$ on $\sigma_{\theta}=26.5 \mathrm{~kg} \mathrm{~m}^{-3} \quad$ JULY

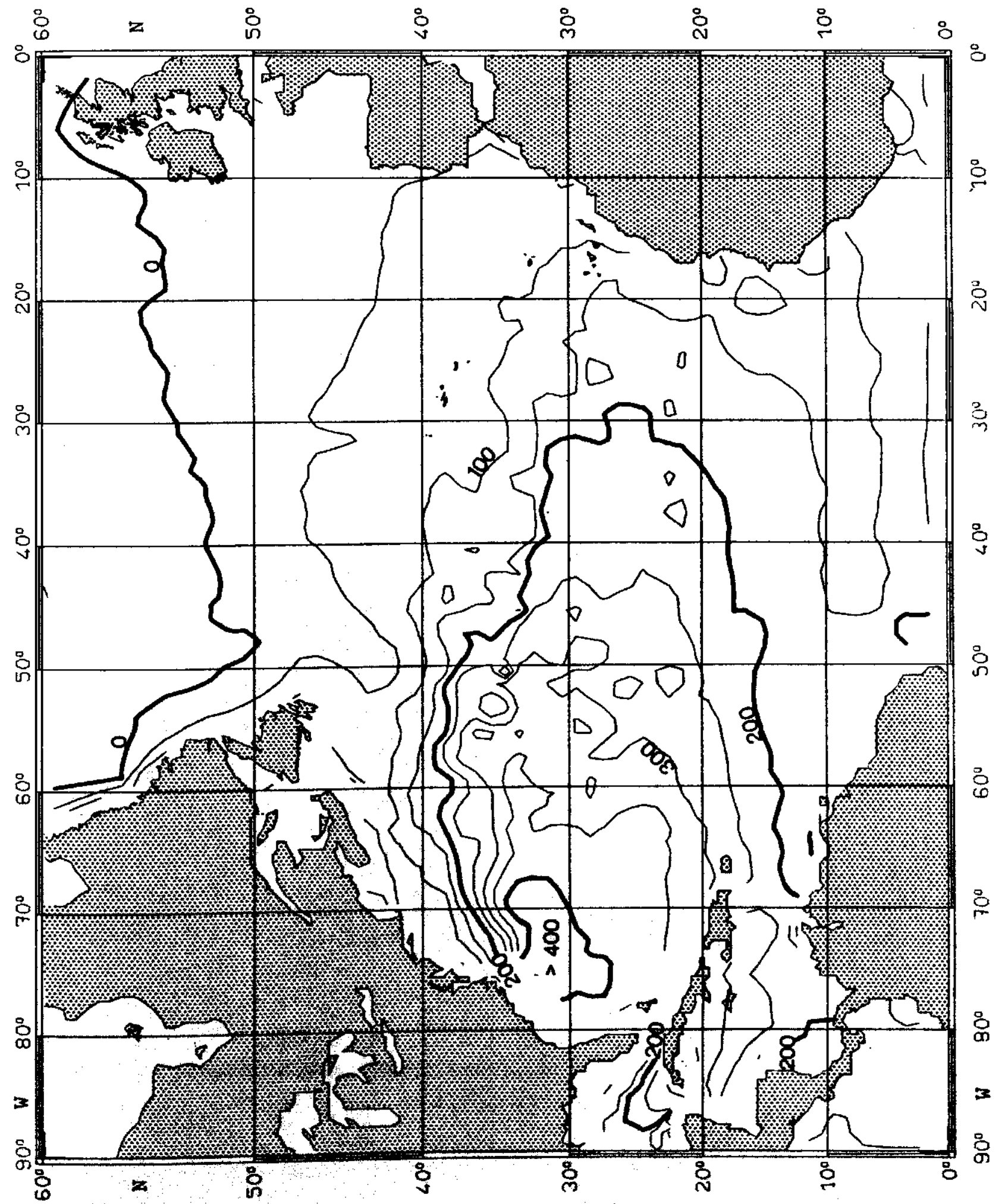


Fig. 80 :

TEMPERATURE $\left({ }^{\circ} \mathrm{C}\right)$ on $\sigma_{\theta}=26.5 \mathrm{~kg} \mathrm{~m}^{-3} \quad$ JULY

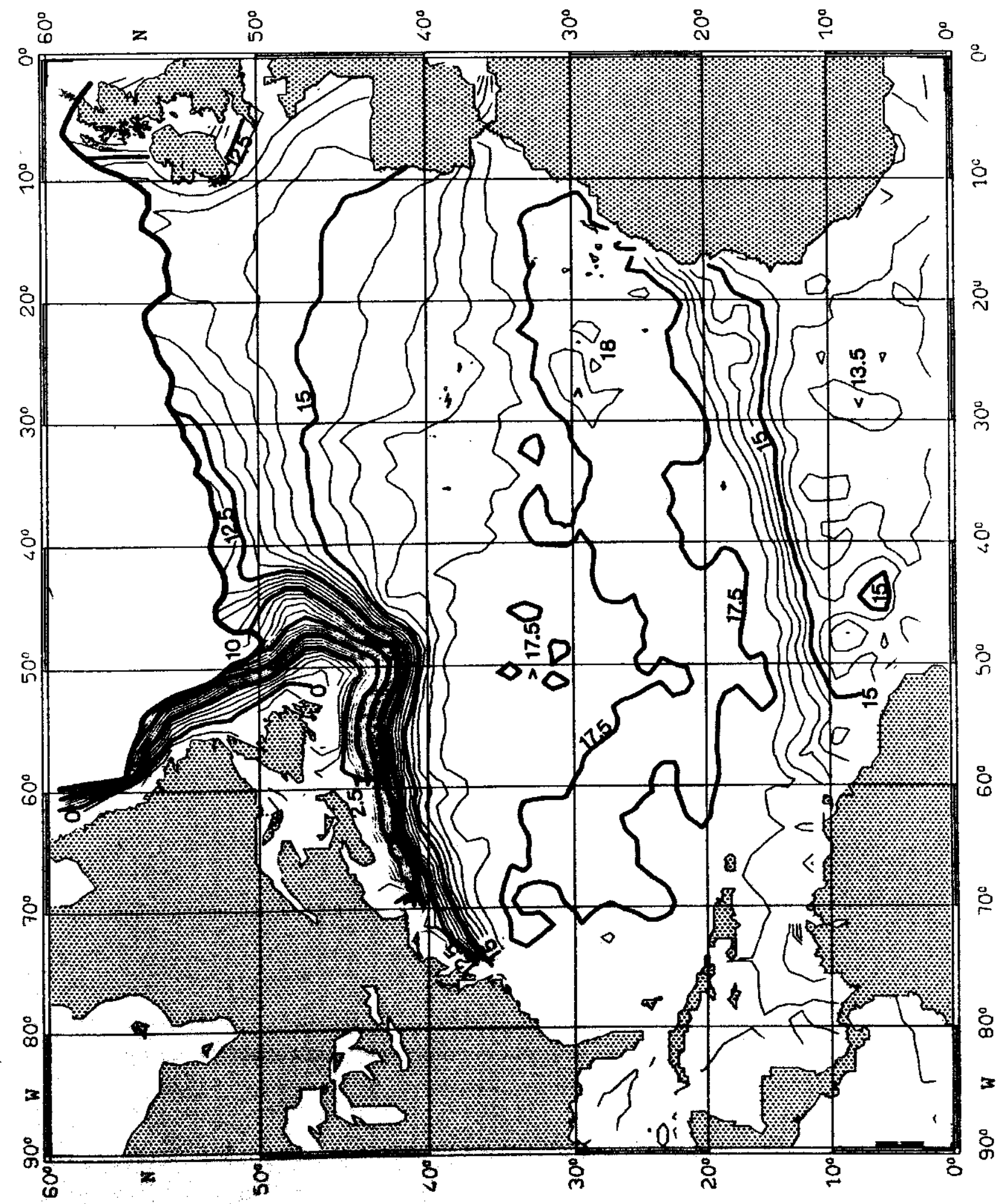


Fig. 81:

SALIMITY $\left(10^{-3}\right)$ on $\sigma_{\theta}=26.5 \mathrm{~kg} \mathrm{~m}^{-3} \quad$ JULY

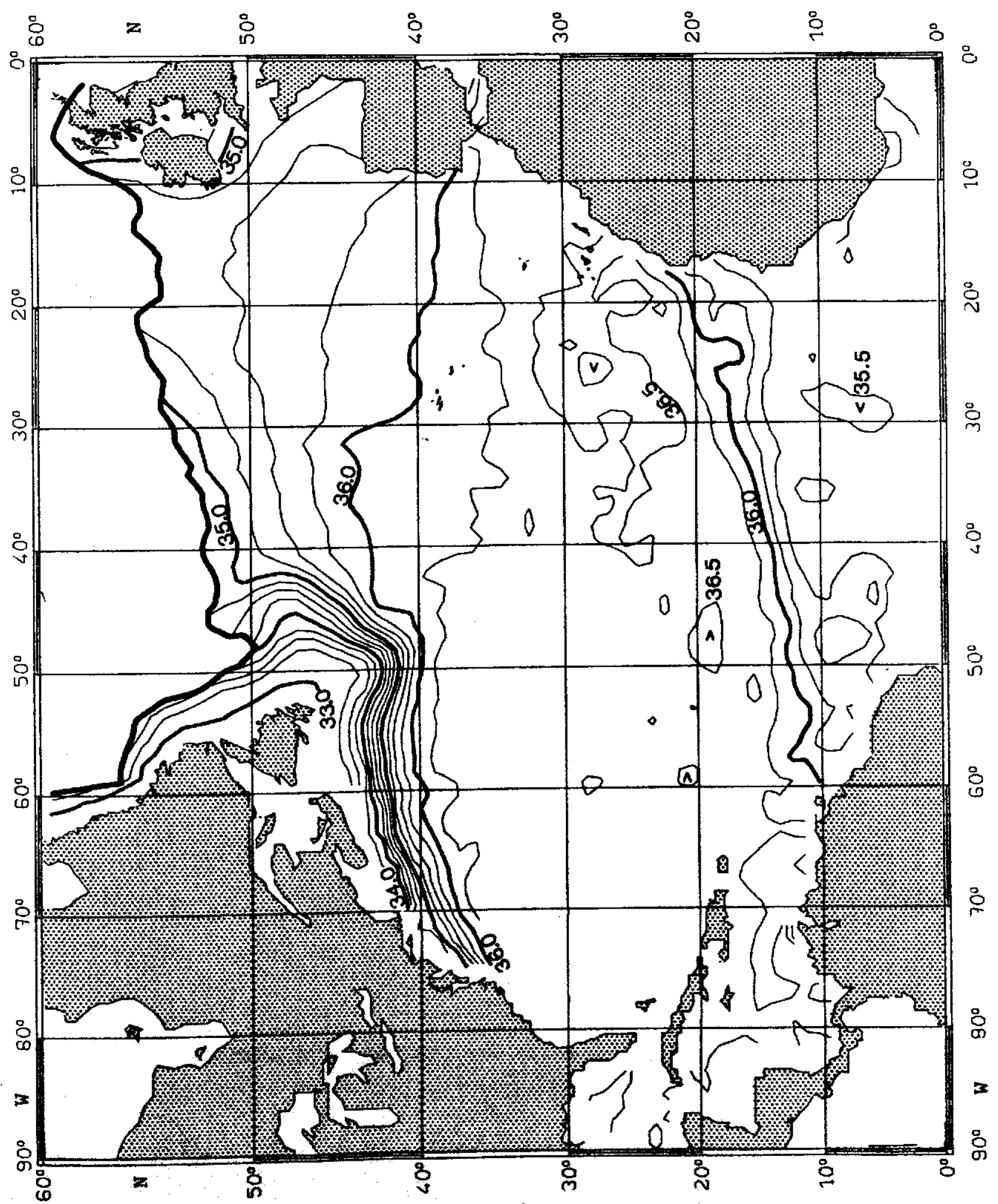


Fig. 82:

PRESSURE $\left(10^{4} \mathrm{~Pa}\right)$ on $\sigma_{\theta}=27.0 \mathrm{~kg} \mathrm{~m}^{-3} \quad$ JULY

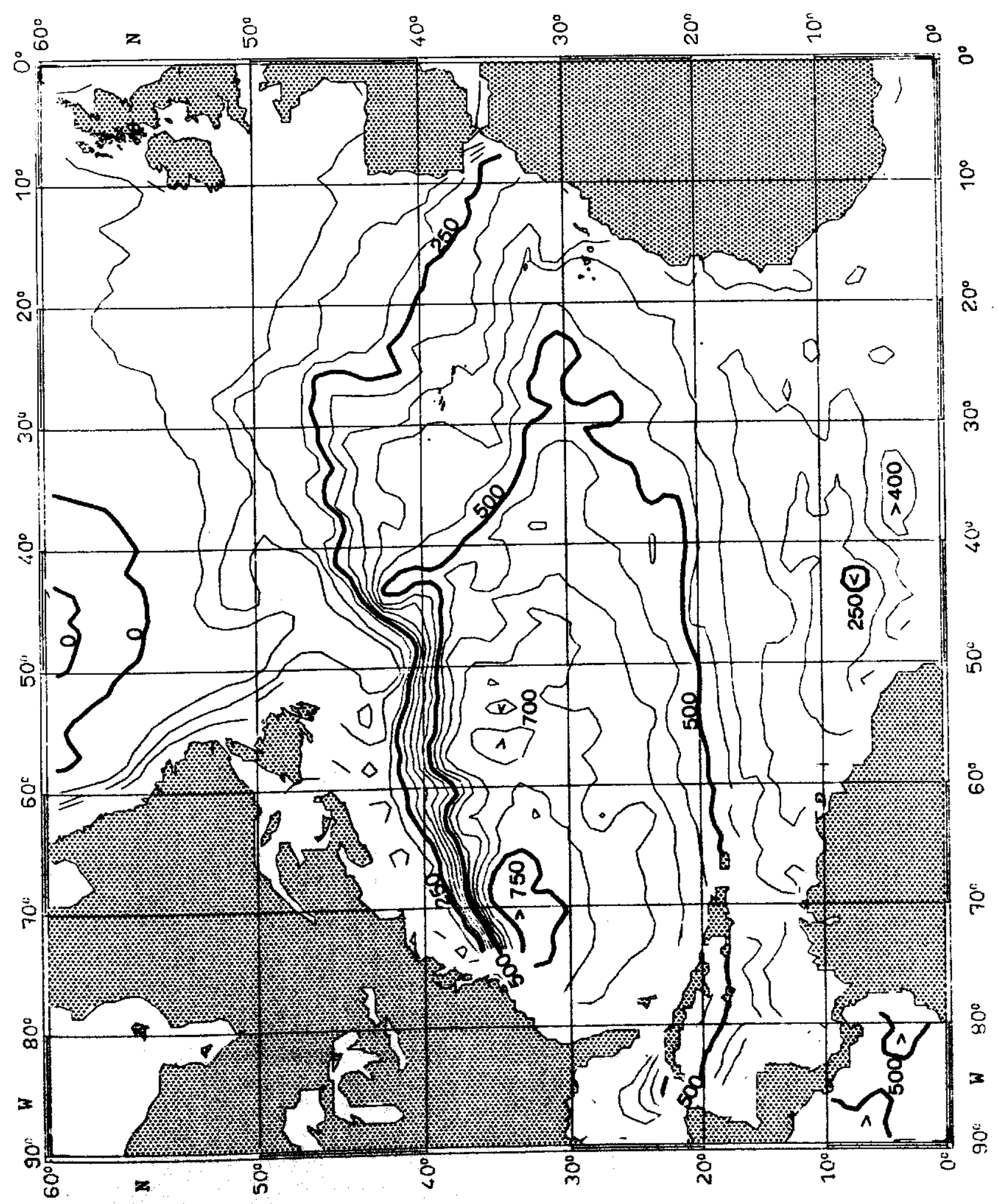


Fig. 83:

TEMPERATURE $\left({ }^{\circ} \mathrm{C}\right)$ on $\sigma_{\theta}=27.0 \mathrm{~kg} \mathrm{~m}^{-3} \quad$ JULY

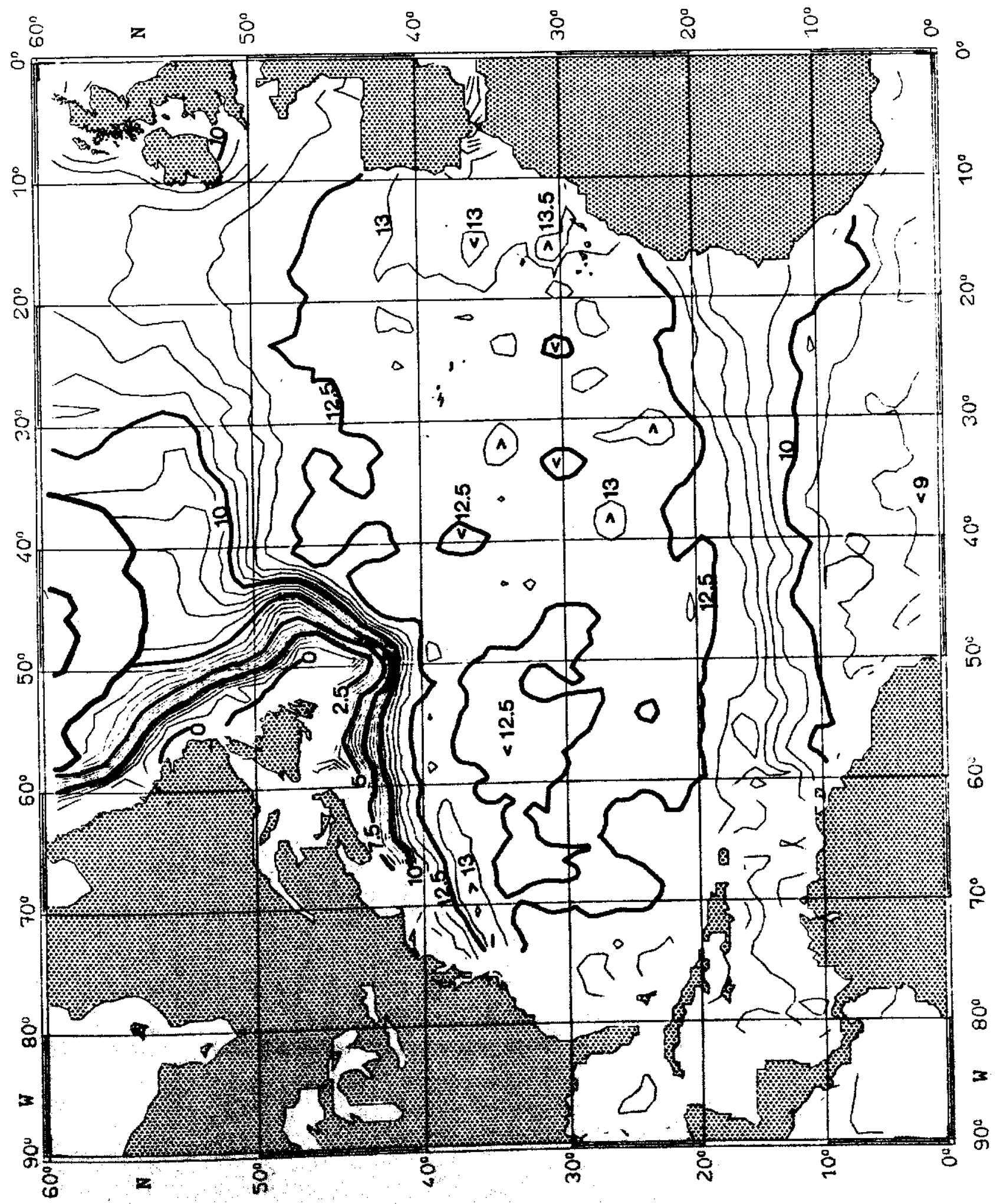


Fig. 84:

SALINITY $\left(10^{-3}\right)$ on $\sigma_{\theta}=27.0 \mathrm{~kg} \mathrm{~m}^{-3} \quad$ JULY

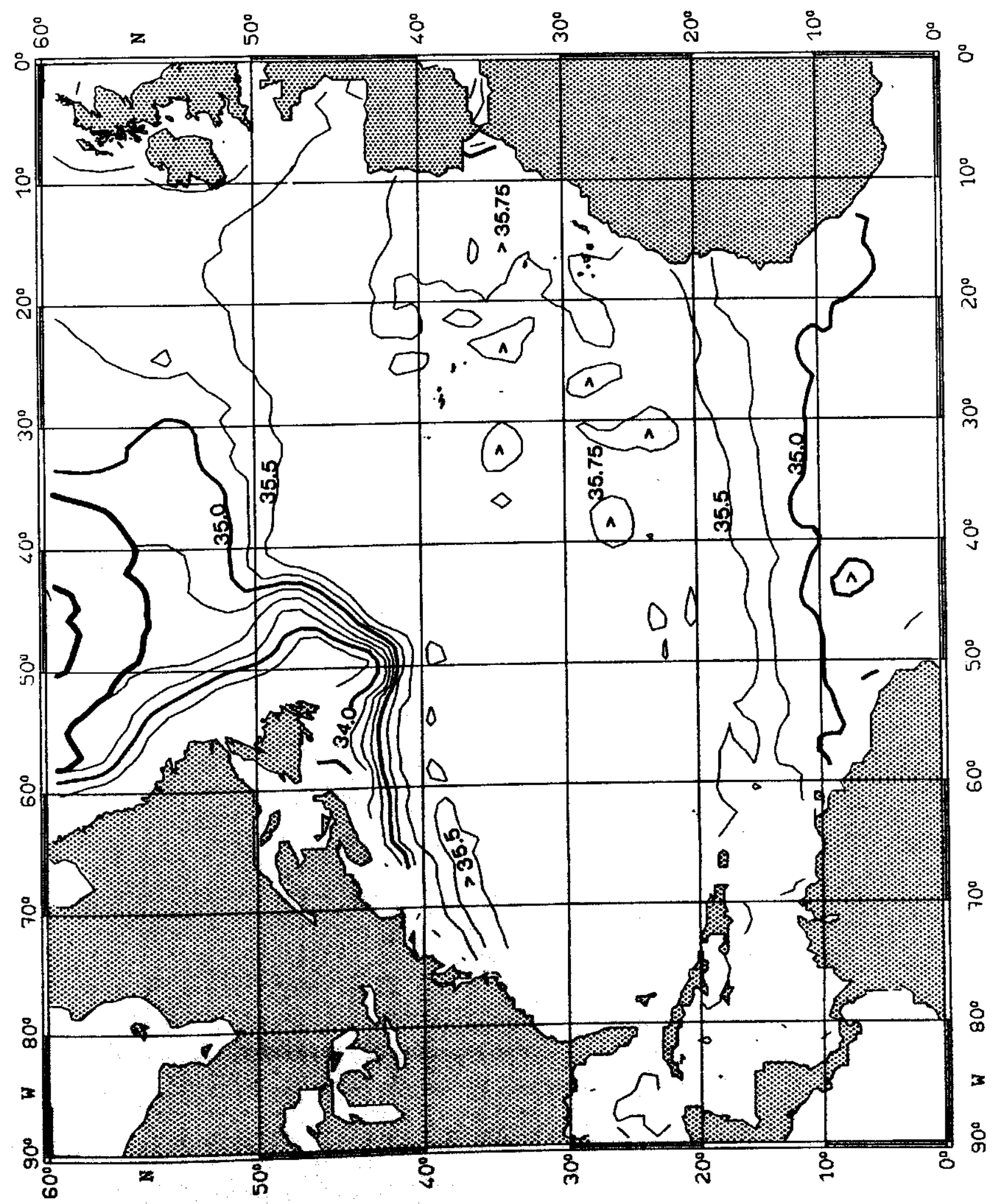


Fig. 85:

PRESSURE $\left(10^{4} \mathrm{~Pa}\right)$ on $\sigma_{\theta}=25.0 \mathrm{~kg} \mathrm{~m}^{-3} \quad$ AUGUST

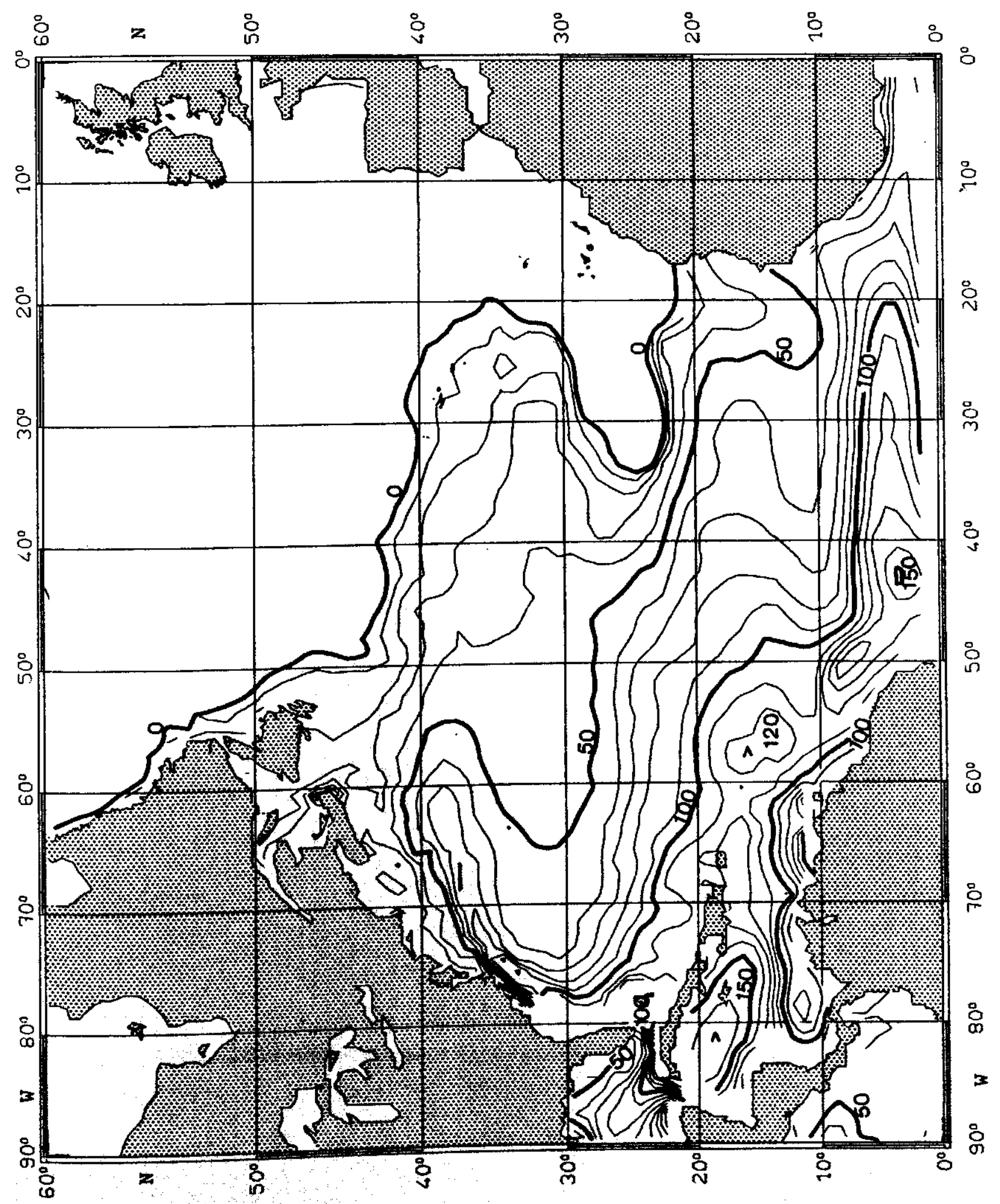


Fig. 86:

TEMPERATURE $\left({ }^{\circ} \mathrm{C}\right)$ on $\sigma_{\theta}=25.0 \mathrm{~kg} \mathrm{~m}^{-3}$ AUGUST

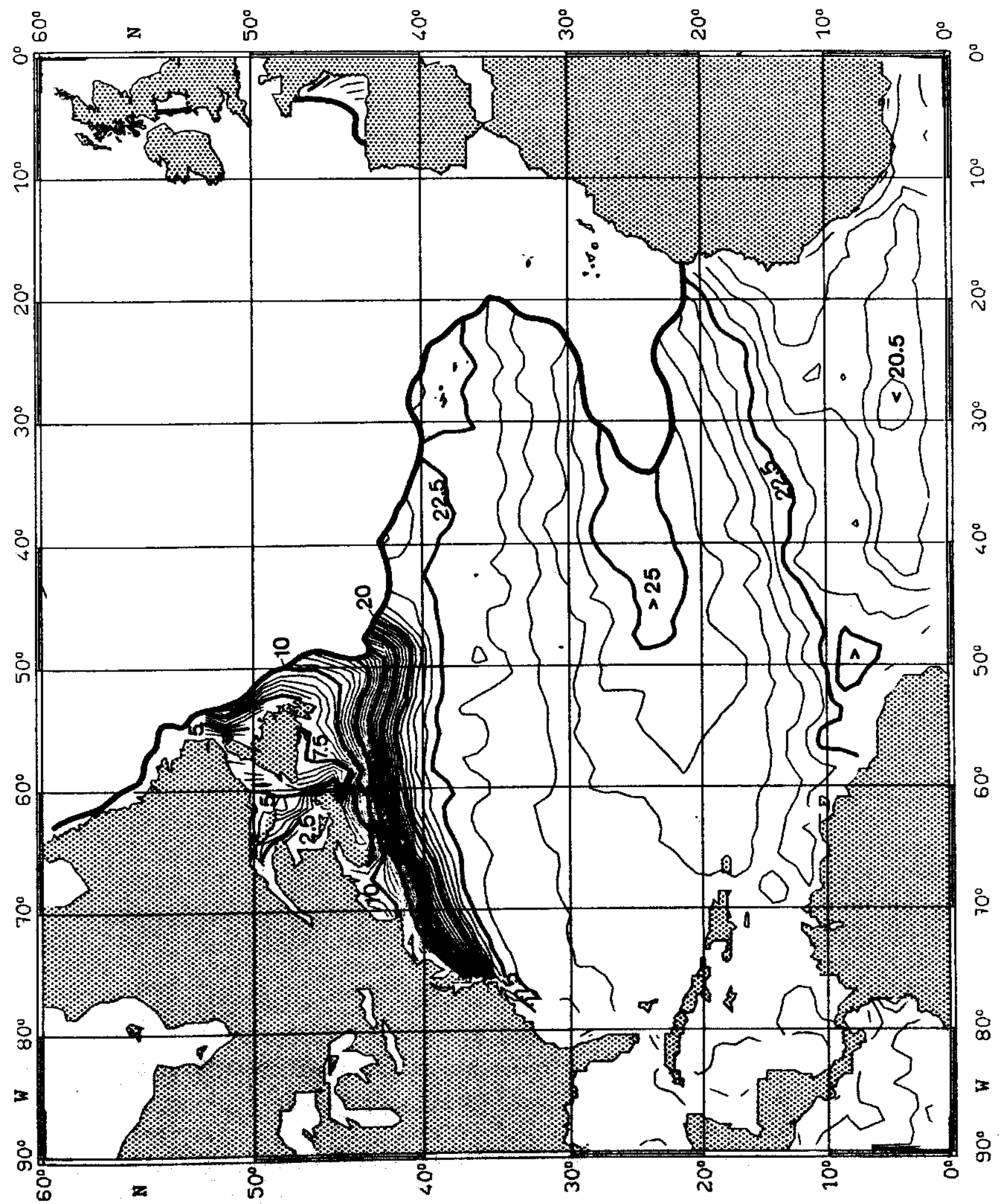


Fig. 87:

SALINITY $\left(10^{-3}\right)$ on $\sigma_{\theta}=25.0 \mathrm{~kg} \mathrm{~m}^{-3} \quad$ AUGUST

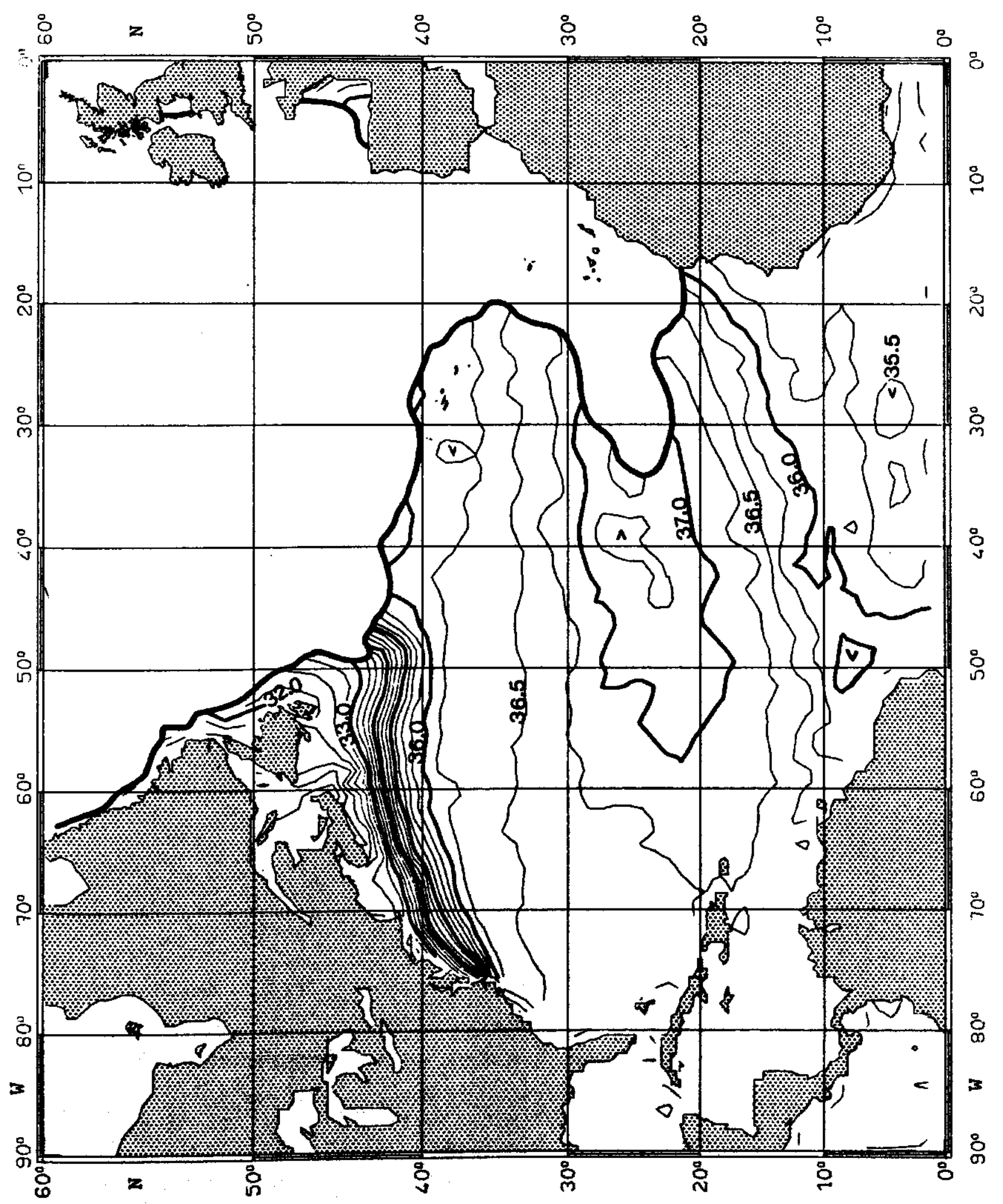


Fig. 88:

PRESSURE $\left(10^{4} \mathrm{~Pa}\right)$ on $\sigma_{\theta}=25.5 \mathrm{~kg} \mathrm{~m}^{-3} \quad$ AUGUST

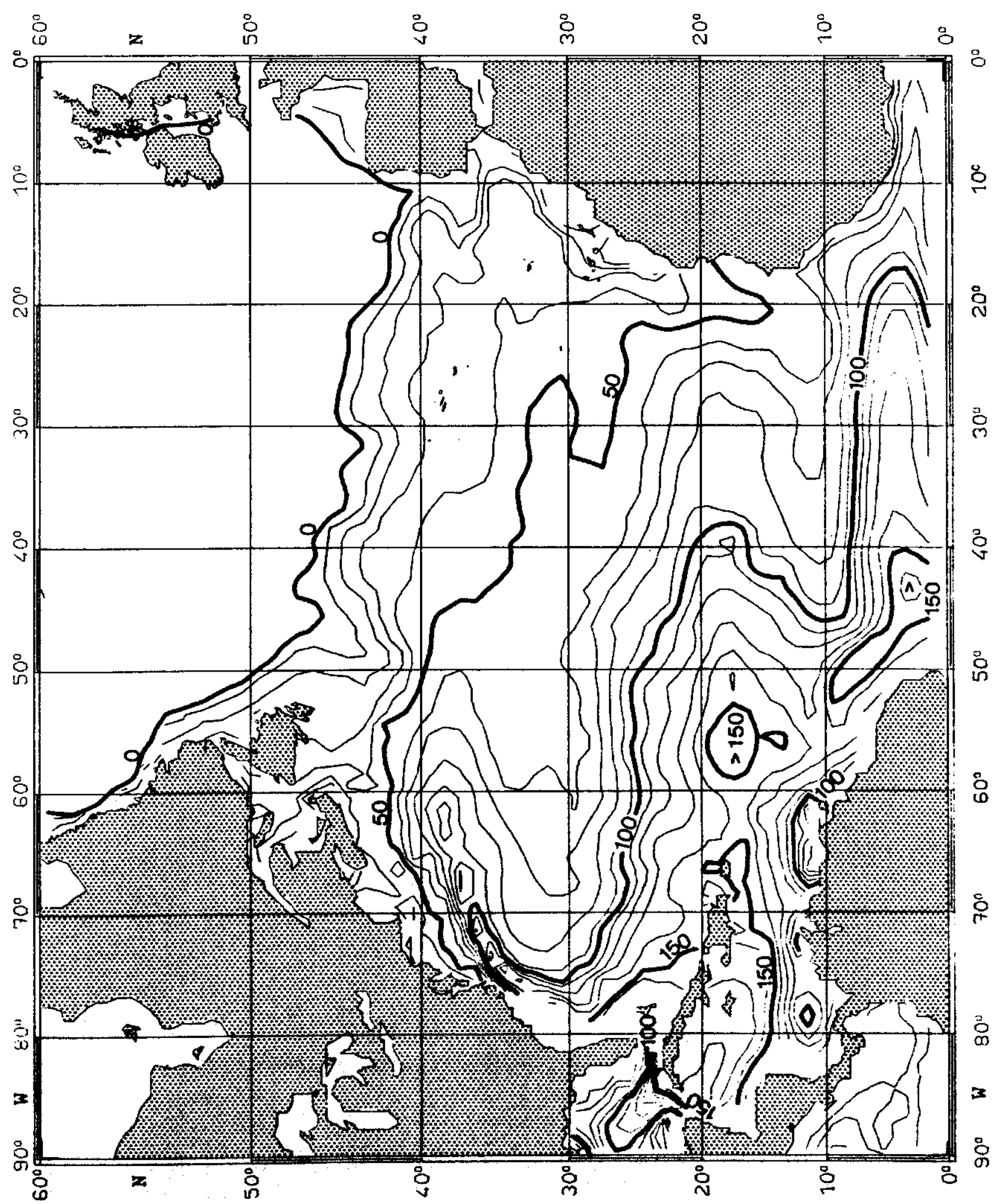


Fig. 89:

TEMPERATURE $\left({ }^{\circ} \mathrm{C}\right)$ on $\sigma_{\theta}=25.5 \mathrm{~kg} \mathrm{~m}^{-3}$ AUGUST

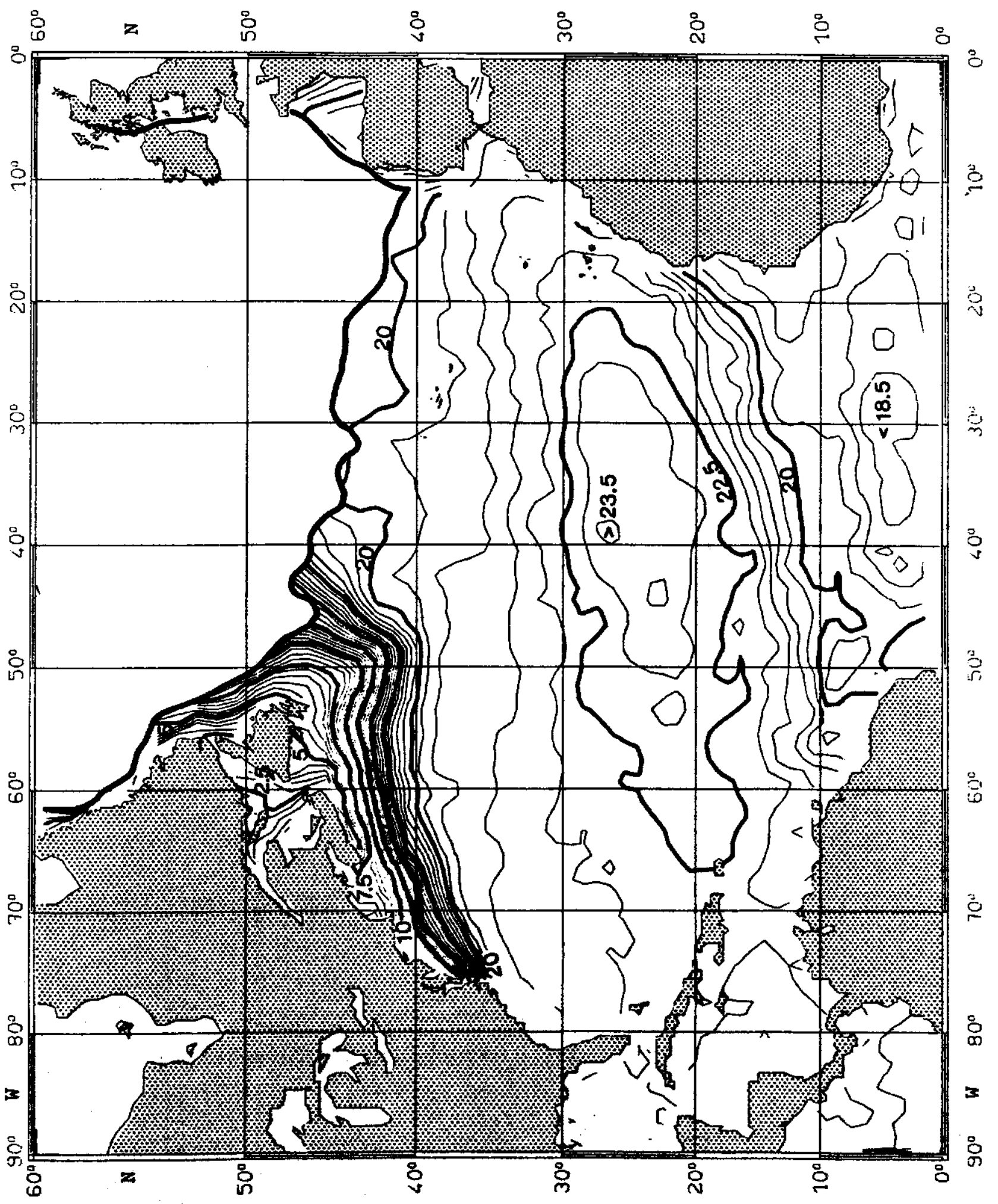


Fig. 90:

SALINITY $\left(10^{-3}\right)$ on $\sigma_{\theta}=25.5 \mathrm{~kg} \mathrm{~m}^{-3} \quad$ AUGUST

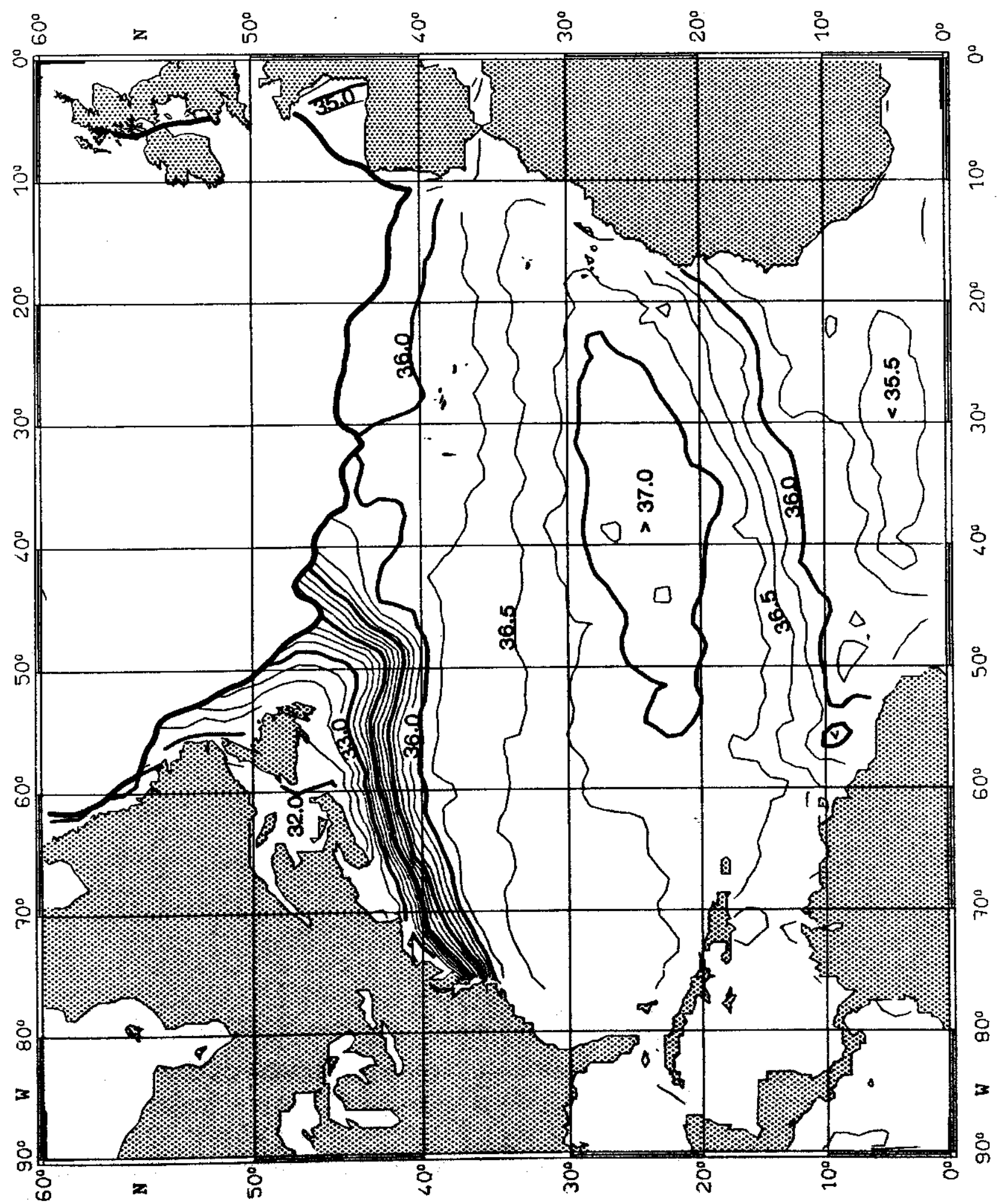


Fig. 91:

PRESSURE $\left(10^{4} \mathrm{~Pa}\right)$ on $\sigma_{\theta}=26.0 \mathrm{~kg} \mathrm{~m}^{-3} \quad$ AUGUST

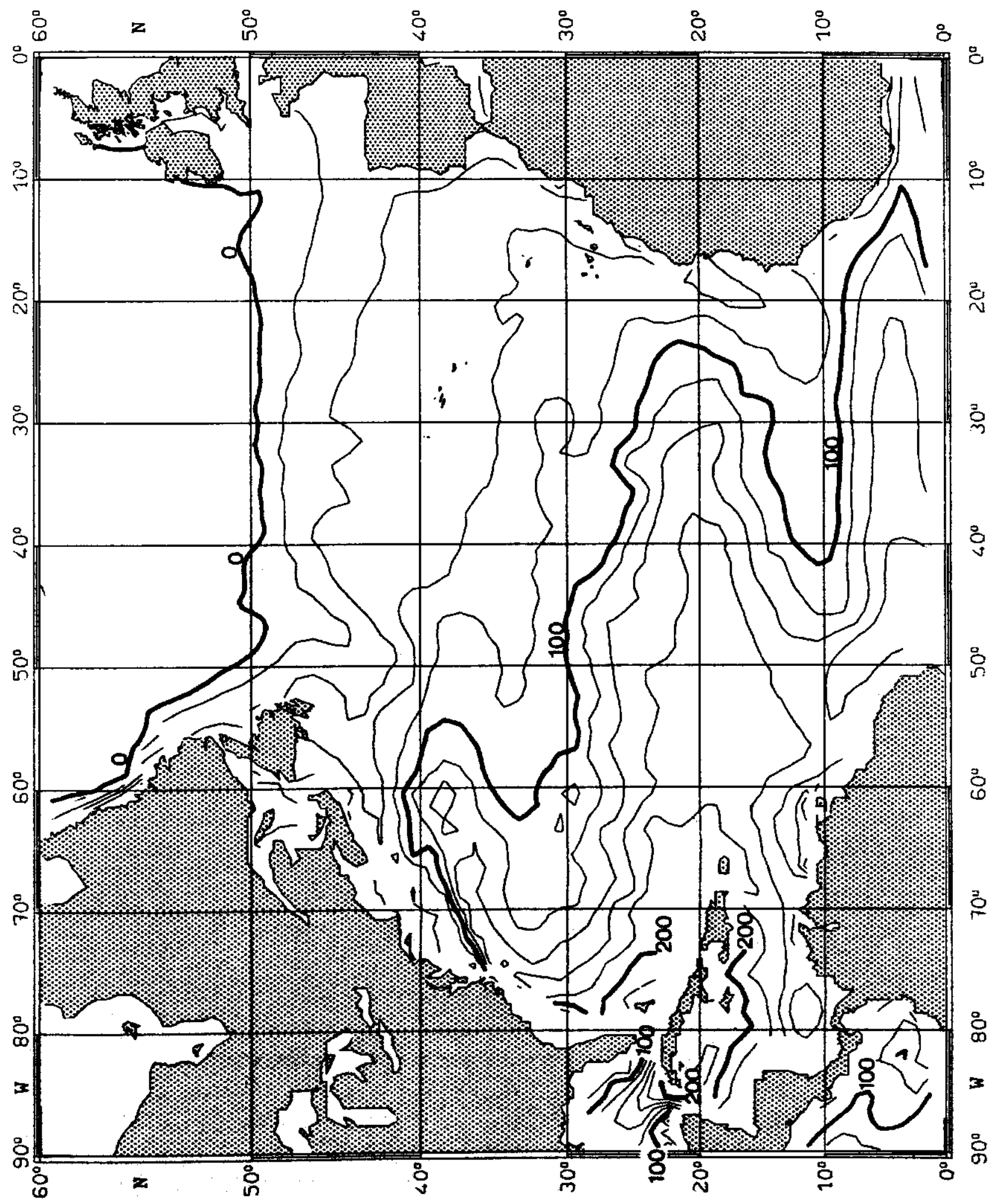


Fig. 92:

TEMPERATURE $\left({ }^{\circ} \mathrm{C}\right)$ on $\sigma_{\theta}=26.0 \mathrm{~kg} \mathrm{~m}^{-3} \quad$ AUGUST

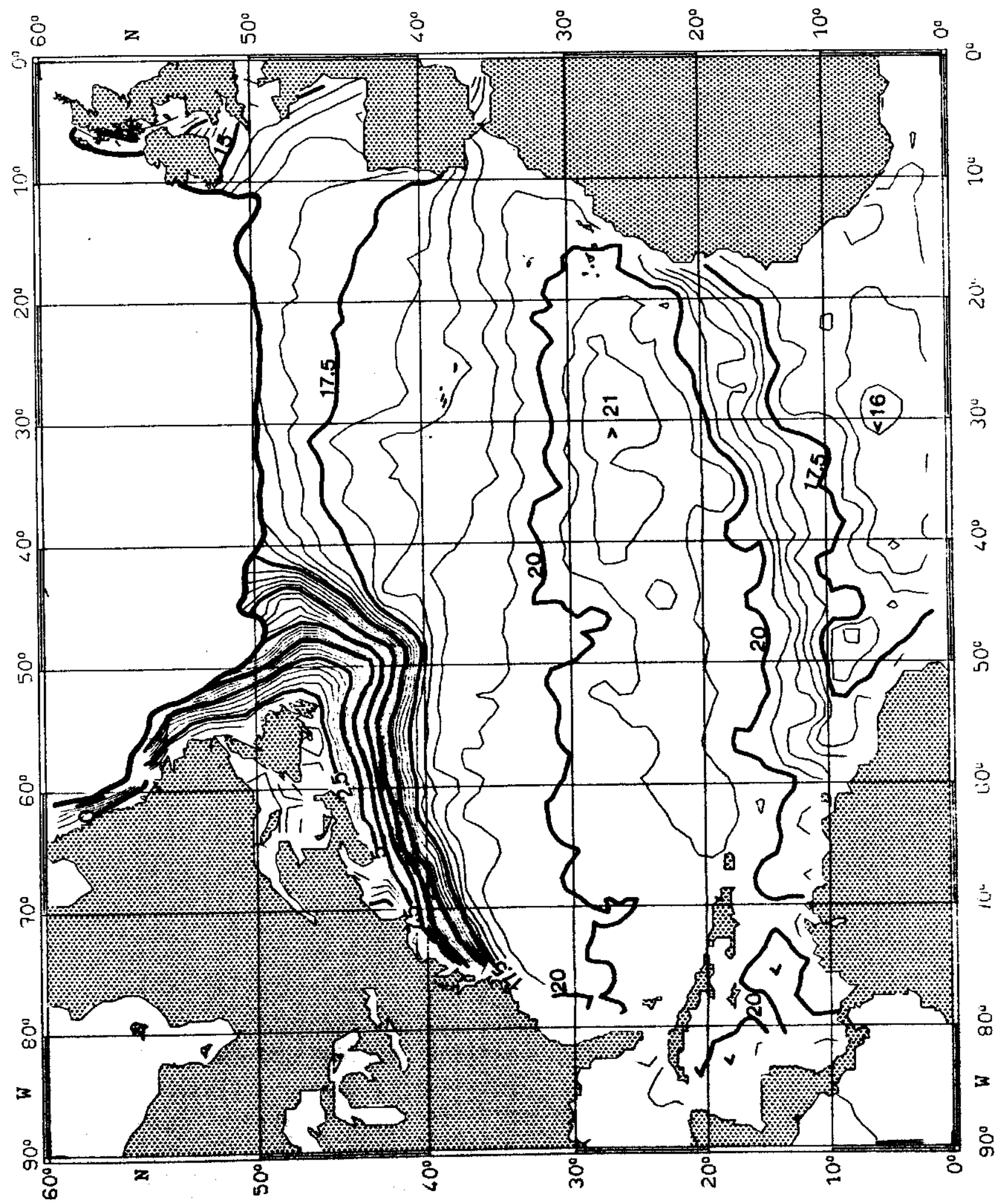


Fig. 93:

SALINITY $\left(10^{-3}\right)$ on $\sigma_{\theta}=26.0 \mathrm{~kg} \mathrm{~m}^{-3} \quad$ AUGUST

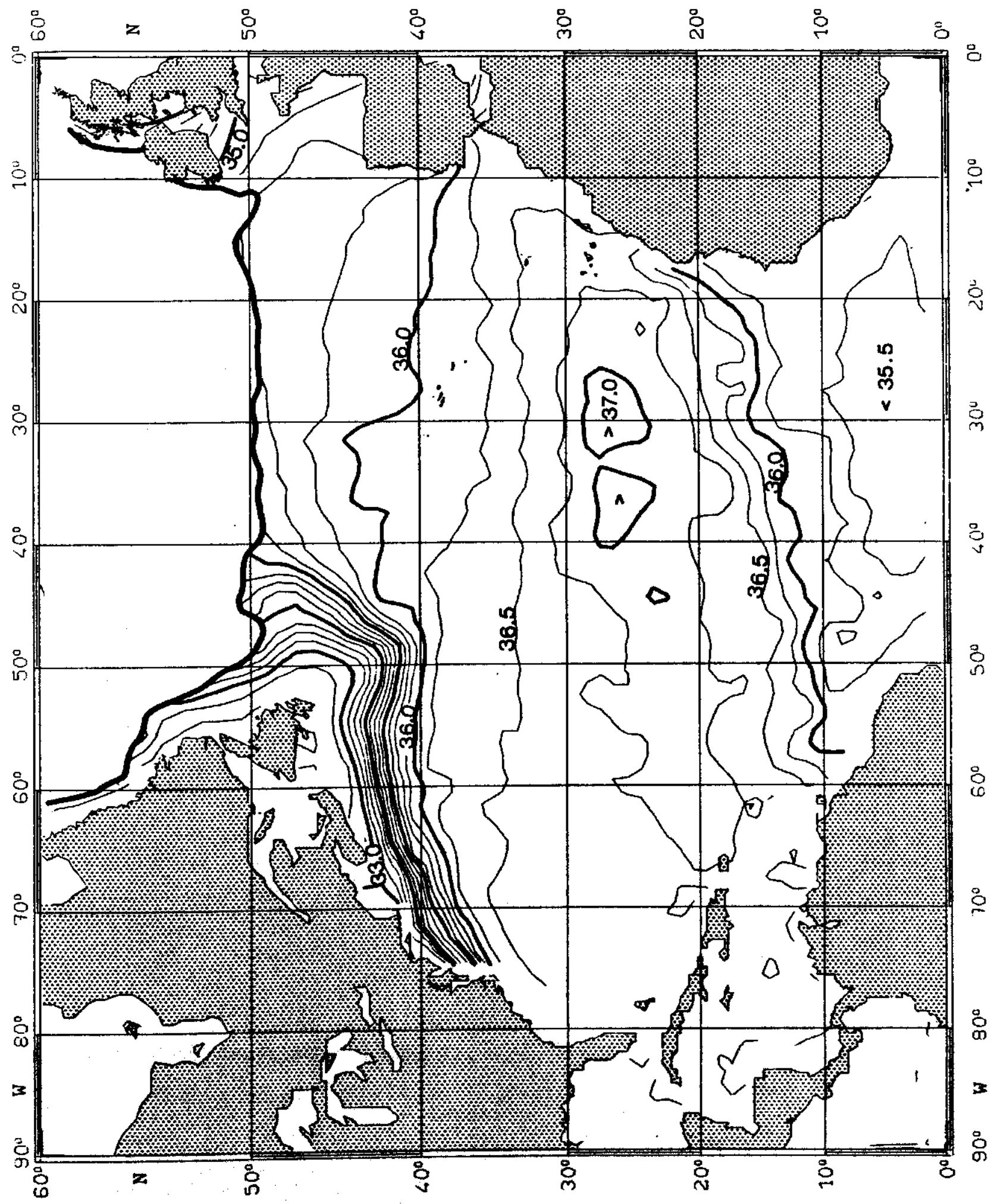


Fig. 94:

PRESSURE $\left(10^{4} \mathrm{~Pa}\right)$ on $\sigma_{\theta}=26.5 \mathrm{~kg} \mathrm{~m}^{-3} \quad$ AUGUST

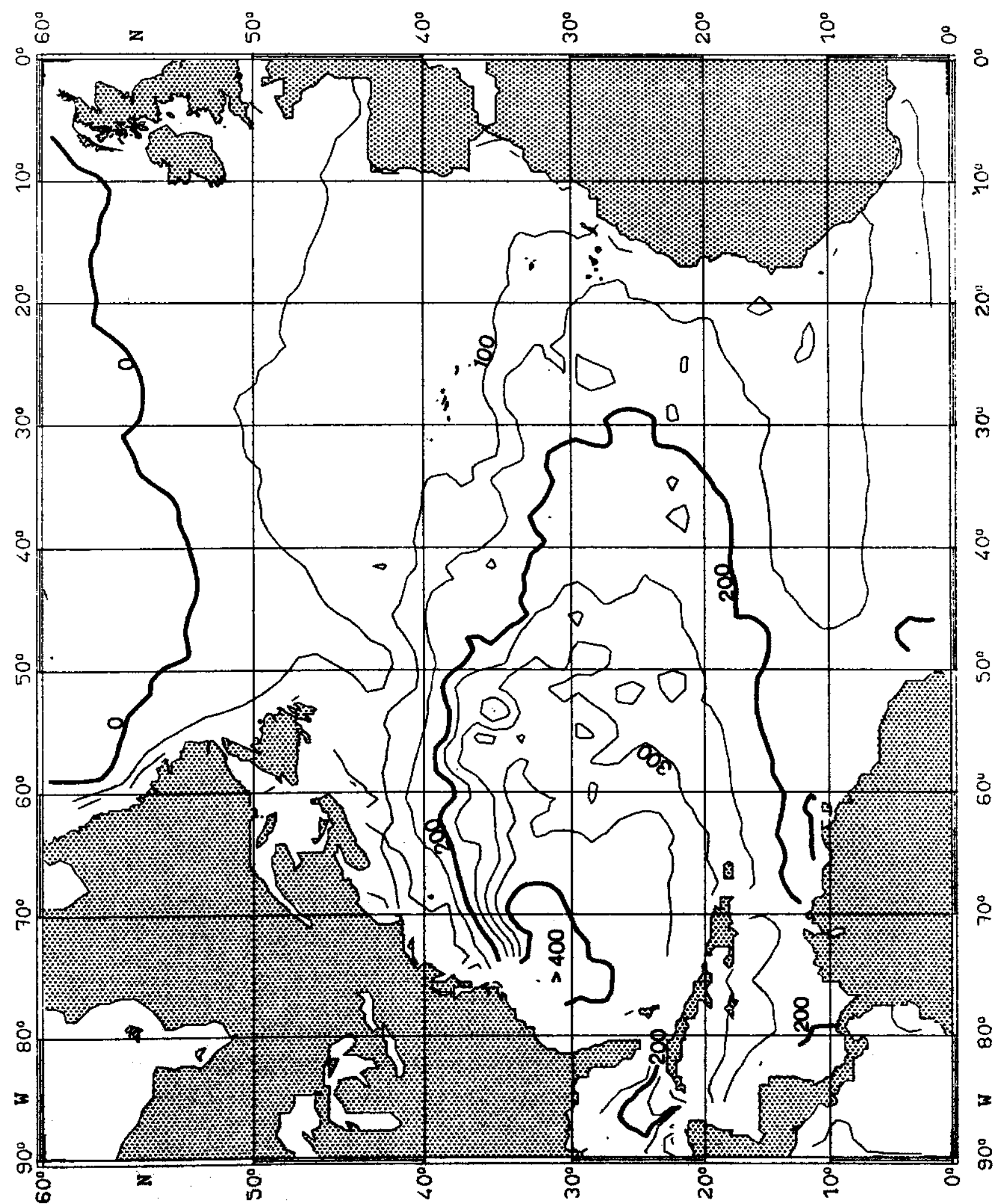


Fig. 95:

TEMPERATURE $\left({ }^{\circ} \mathrm{C}\right)$ on $\sigma_{\theta}=26.5 \mathrm{~kg} \mathrm{~m}^{-3} \quad$ AUGUST

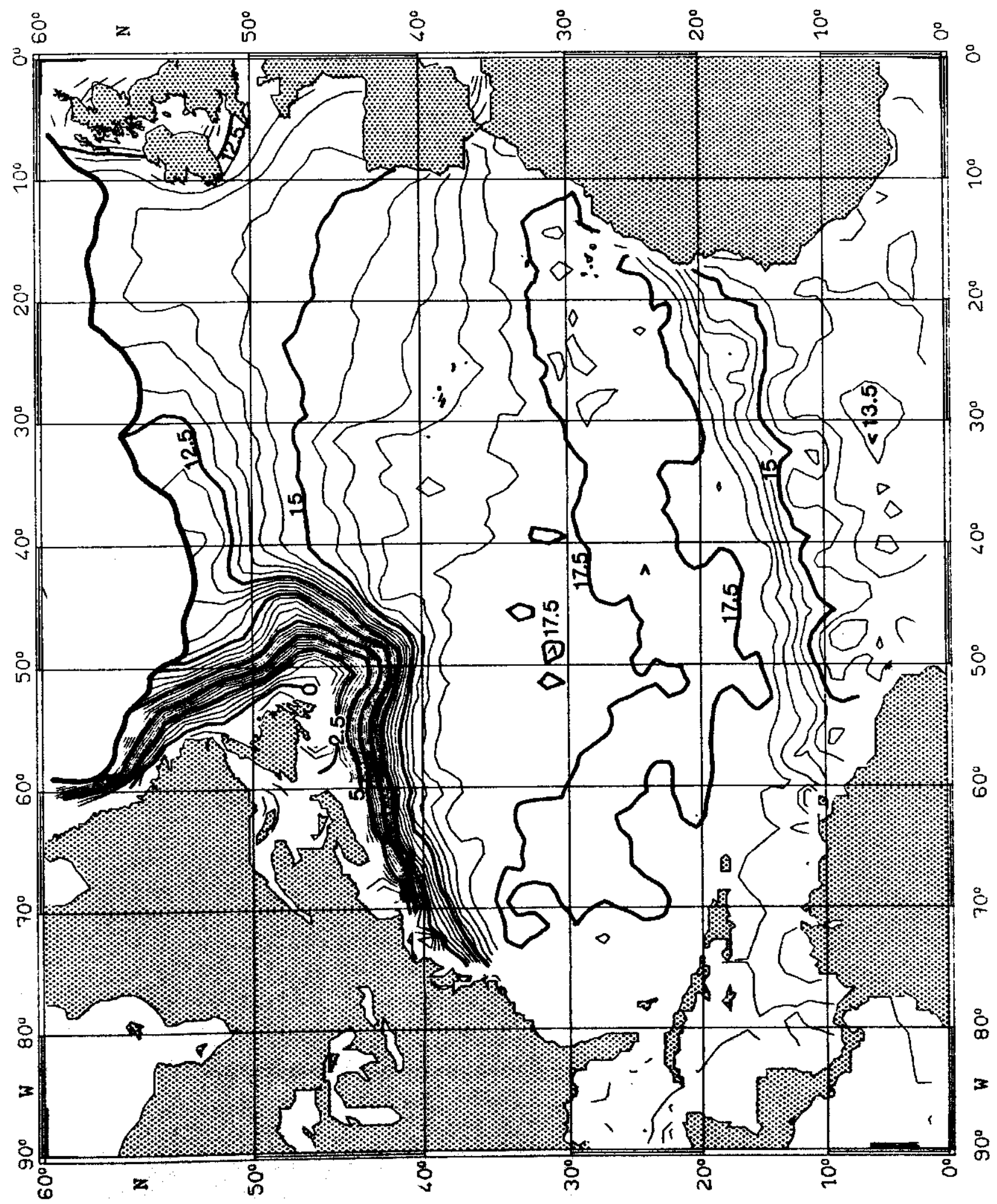


Fig. 96:

SALINITY $\left(10^{-3}\right)$ on $\sigma_{\theta}=26.5 \mathrm{~kg} \mathrm{~m}^{-3} \quad$ AUGUST

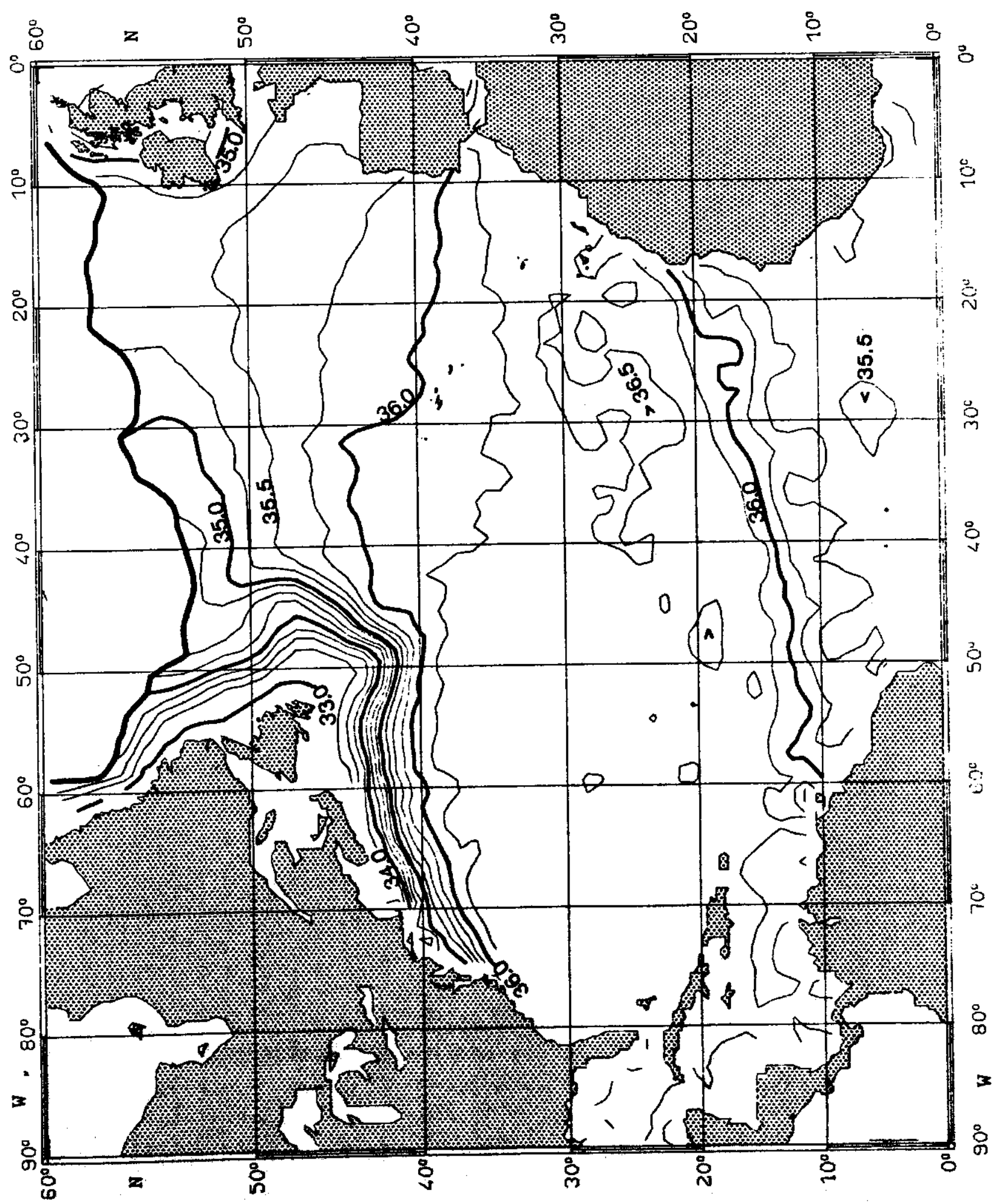


Fig. 97:

PRESSURE $\left(10^{4} \mathrm{~Pa}\right)$ on $\sigma_{\theta}=27.0 \mathrm{~kg} \mathrm{~m}^{-3} \quad$ AUGUST

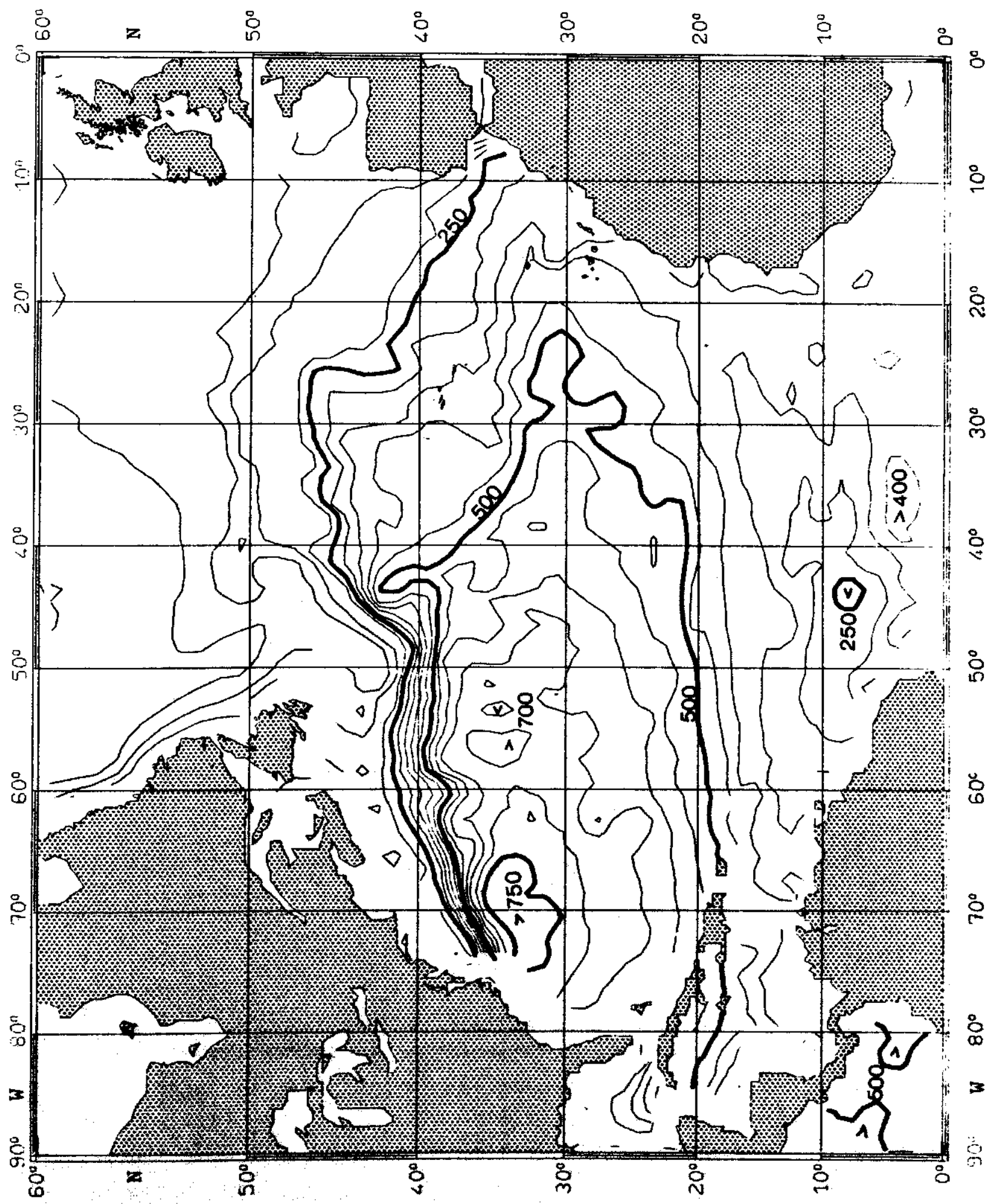


Fig. 98:

TEMPERATURE $\left({ }^{\circ} \mathrm{C}\right)$ on $\sigma_{\theta}=27.0 \mathrm{~kg} \mathrm{~m}^{-3} \quad$ AUGUST

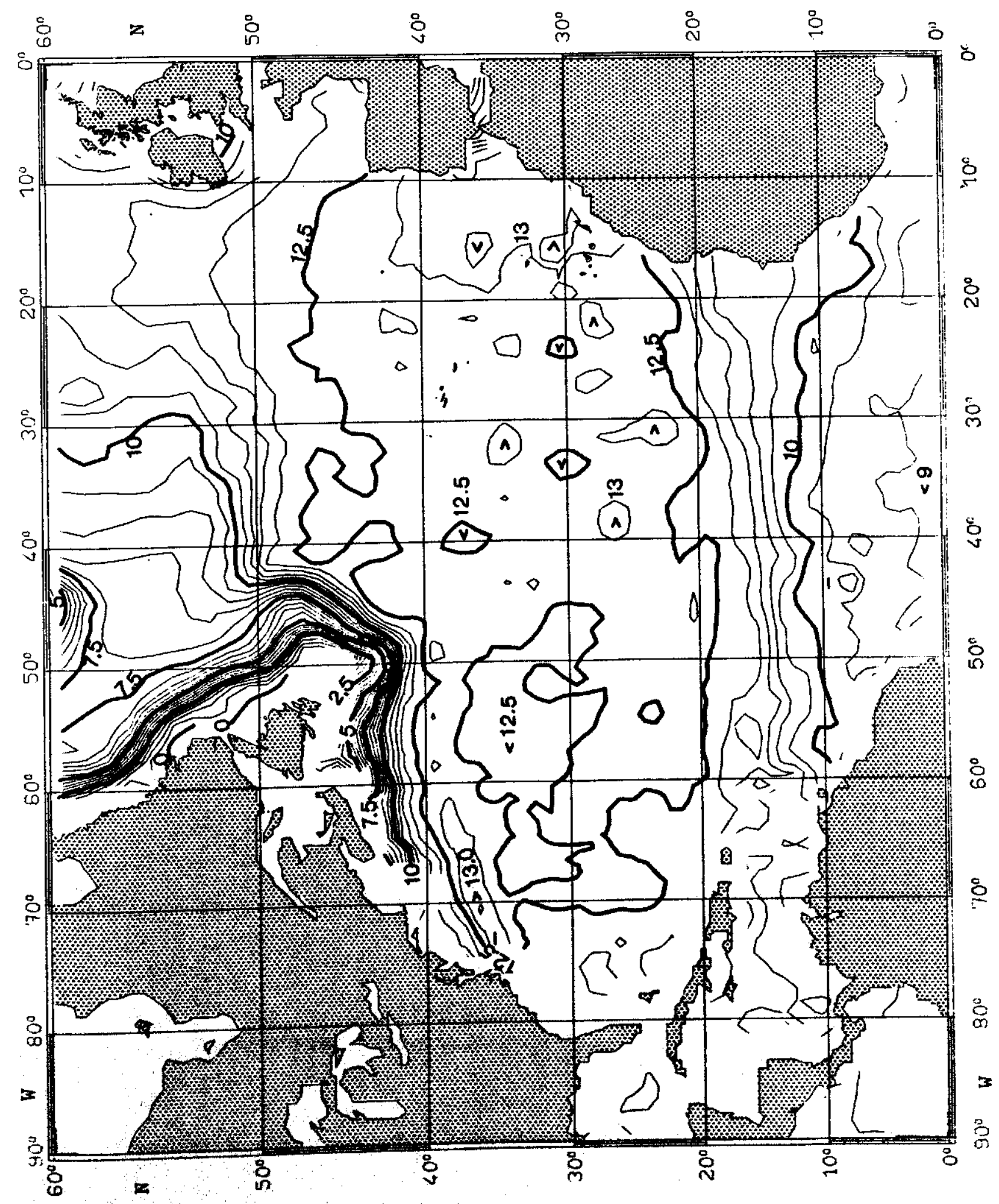


Fig. 99:

SALINITY $\left(10^{-3}\right)$ on $\sigma_{\theta}=27.0 \mathrm{~kg} \mathrm{~m}^{-3} \quad$ AUGUST

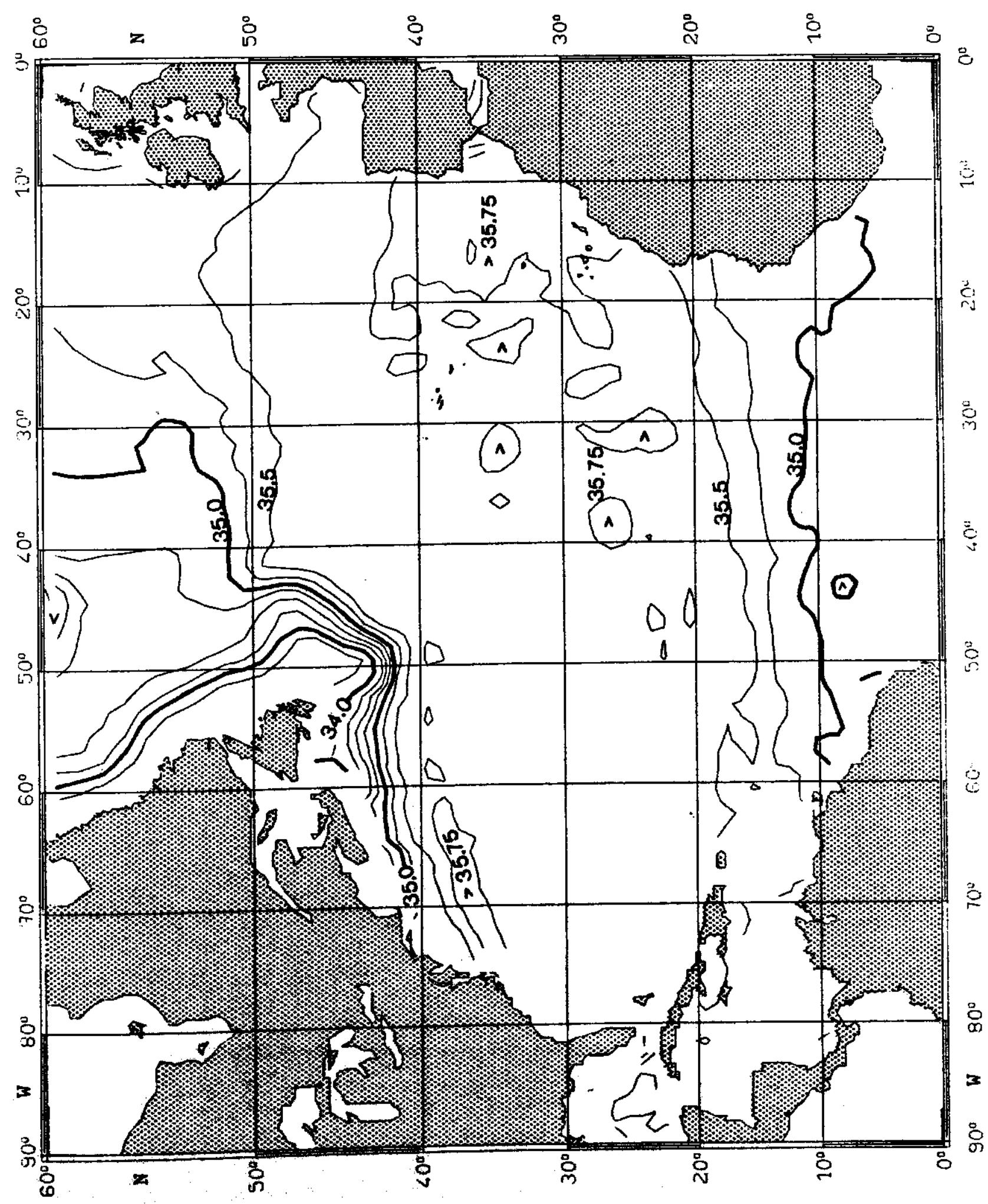


Fig. 100:

PRESSURE $\left(10^{4} \mathrm{~Pa}\right)$ on $\sigma_{\theta}=25.0 \mathrm{~kg} \mathrm{~m}^{-3} \quad$ SEPTEMBER

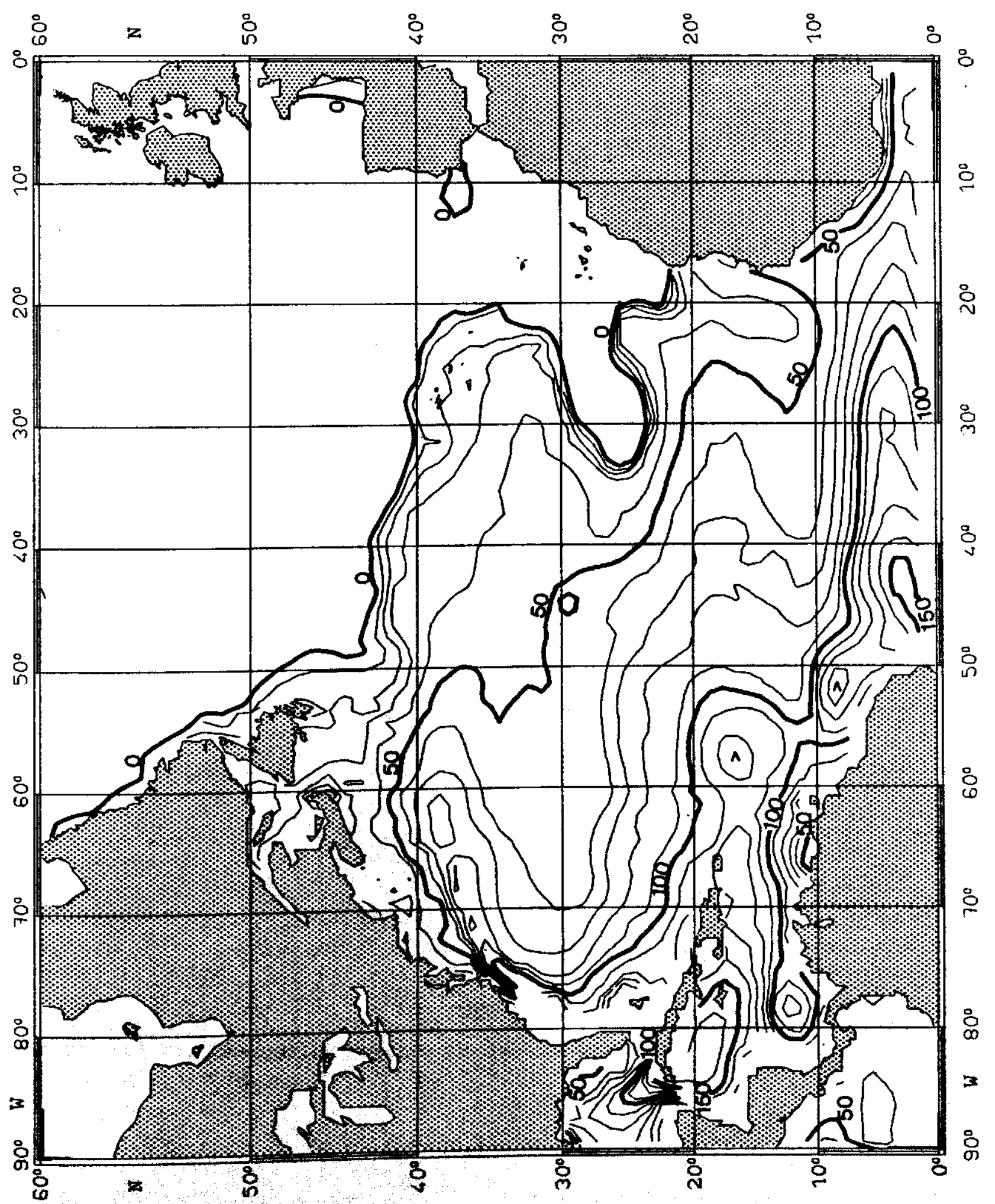


Fig. 101:

TEMPERATURE $\left({ }^{\circ} \mathrm{C}\right)$ on $\sigma_{\theta}=25.0 \mathrm{~kg} \mathrm{~m}^{-3}$ SEPTEMBER

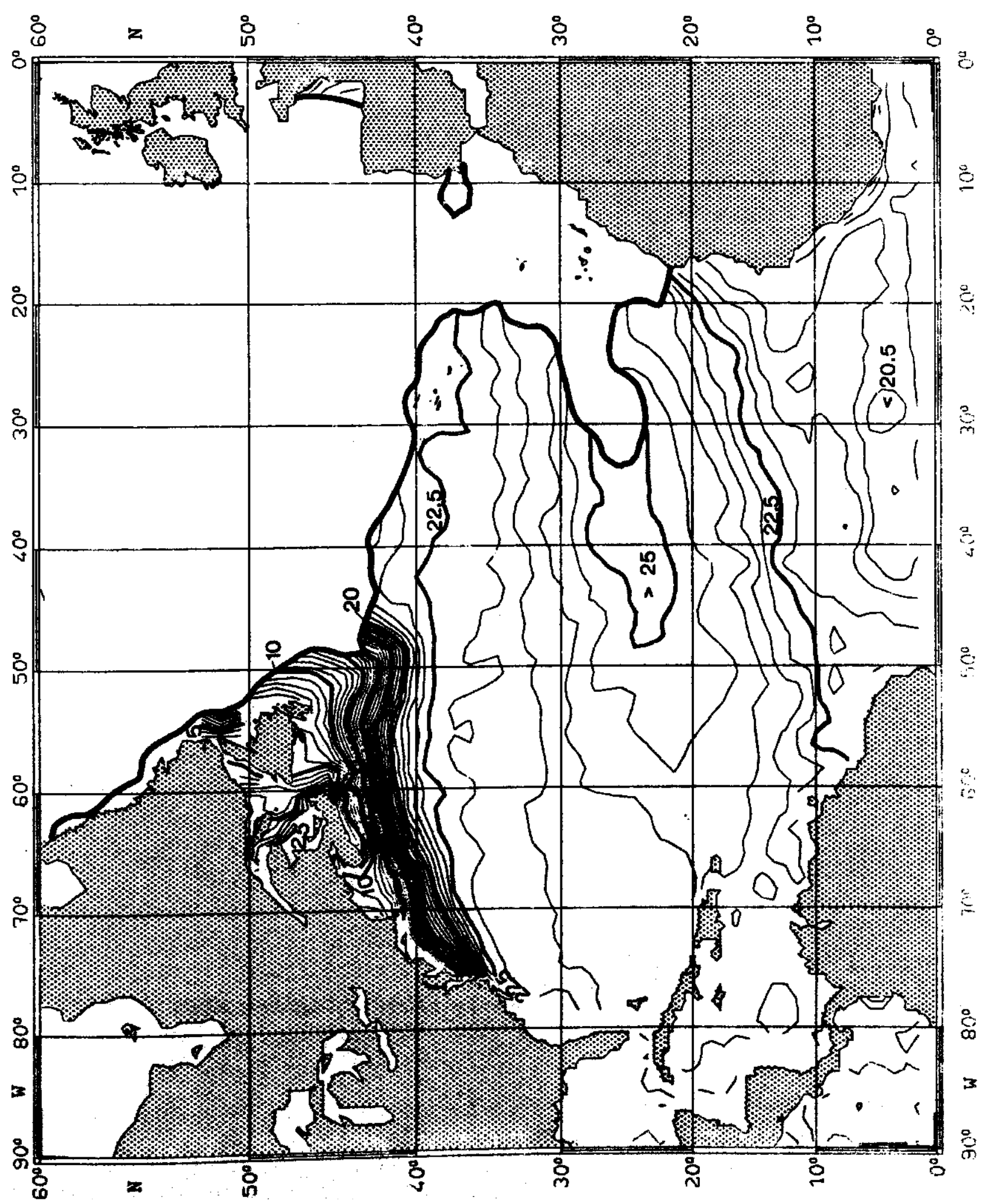


Fig. 102:

SALINITY $\left(10^{-3}\right)$ on $\sigma_{\theta}=25.0 \mathrm{~kg} \mathrm{~m}^{-3} \quad$ SEPTEMBER

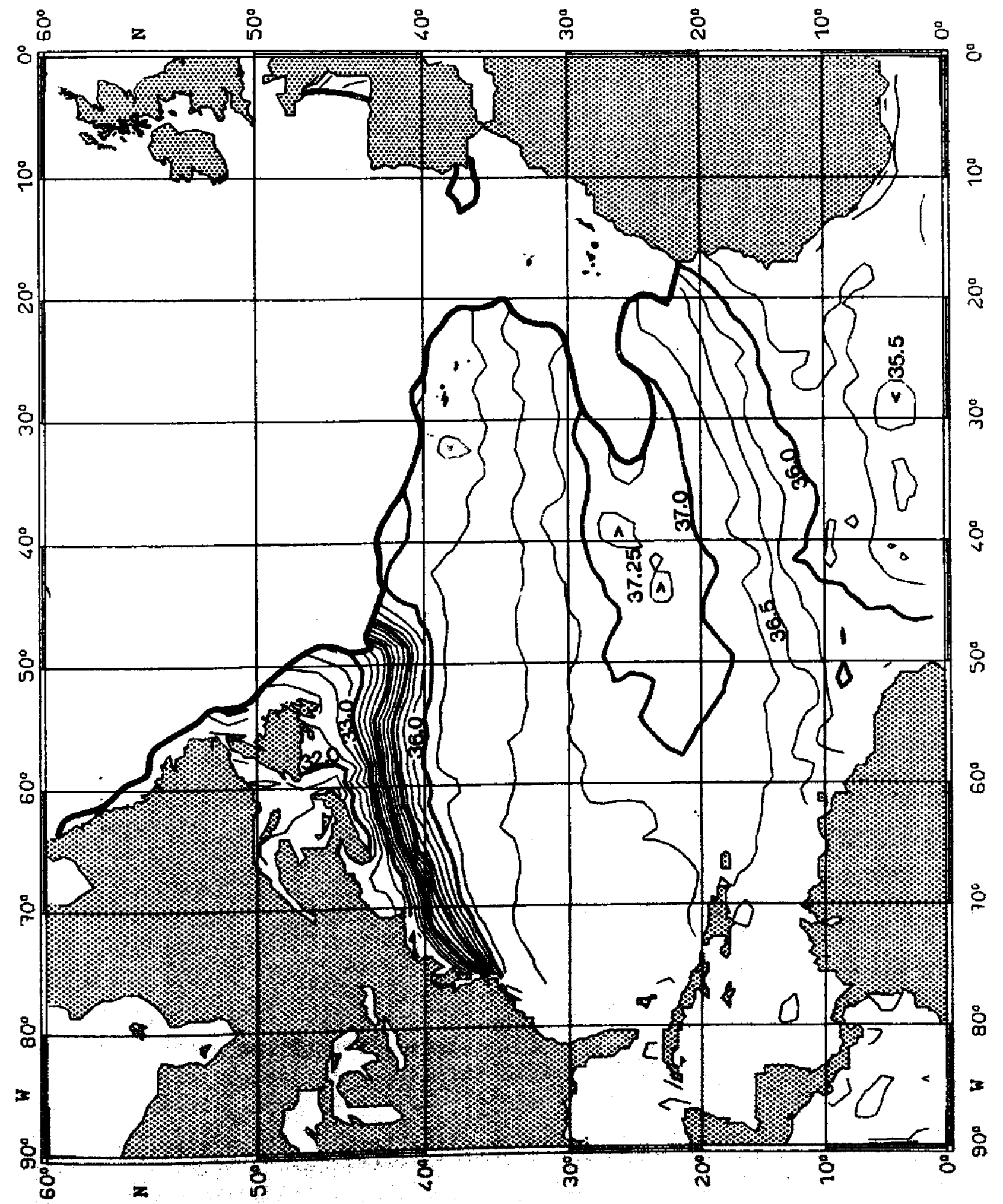


Fig. 103:

PRESSURE $\left(10^{4} \mathrm{~Pa}\right)$ on $\sigma_{\theta}=26.0 \mathrm{~kg} \mathrm{~m}^{-3}$ SEPTEMBER

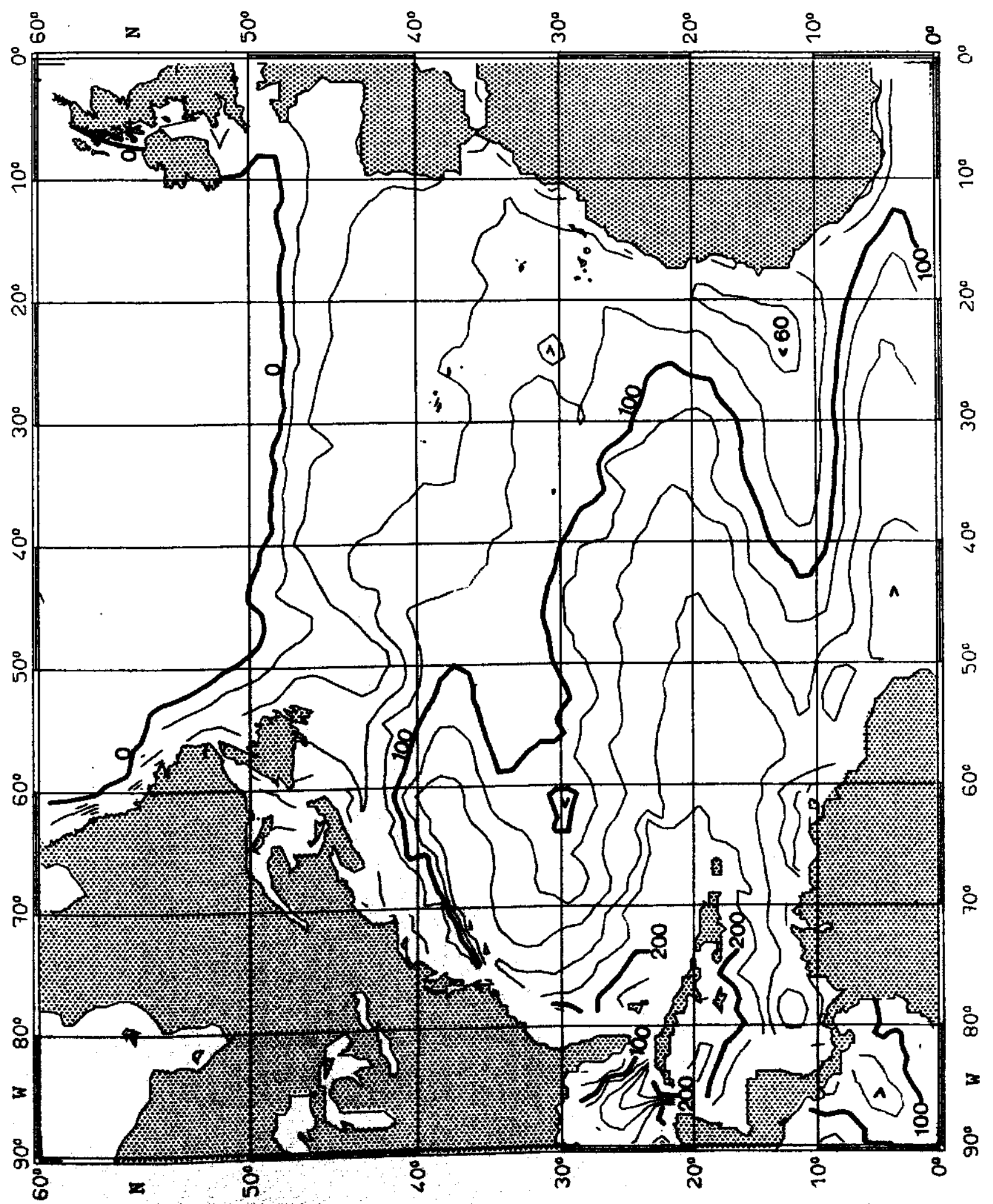


Fig. 104:

TEMPERATURE $\left({ }^{\circ} \mathrm{C}\right)$ on $\sigma_{\theta}=26.0 \mathrm{~kg} \mathrm{~m}^{-3}$ SEPTEMBER

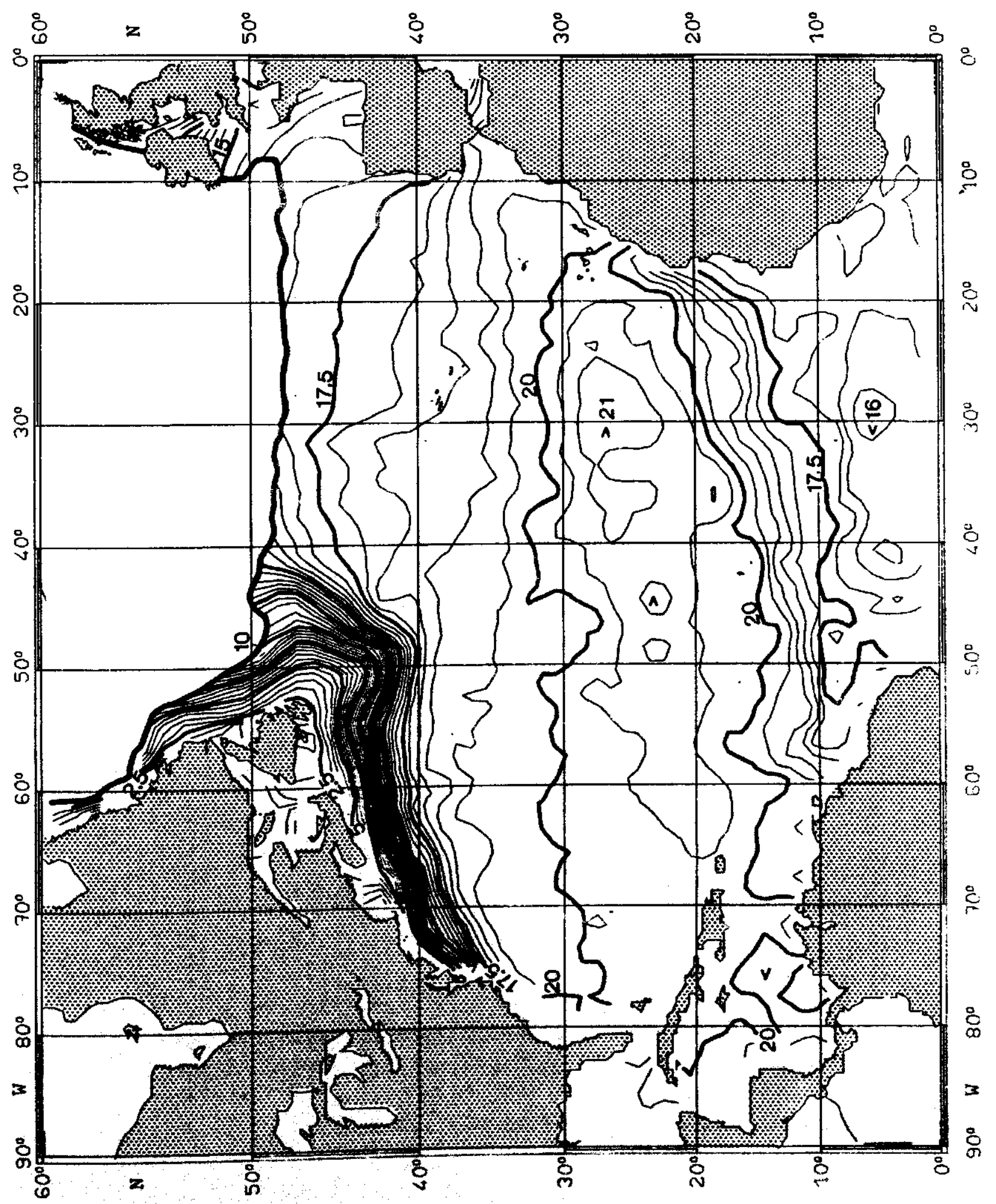


Fig. 105:

SALINITY $\left(10^{-3}\right)$ on $\sigma_{\theta}=26.0 \mathrm{~kg} \mathrm{~m}^{-3} \quad$ SEPTEMBER

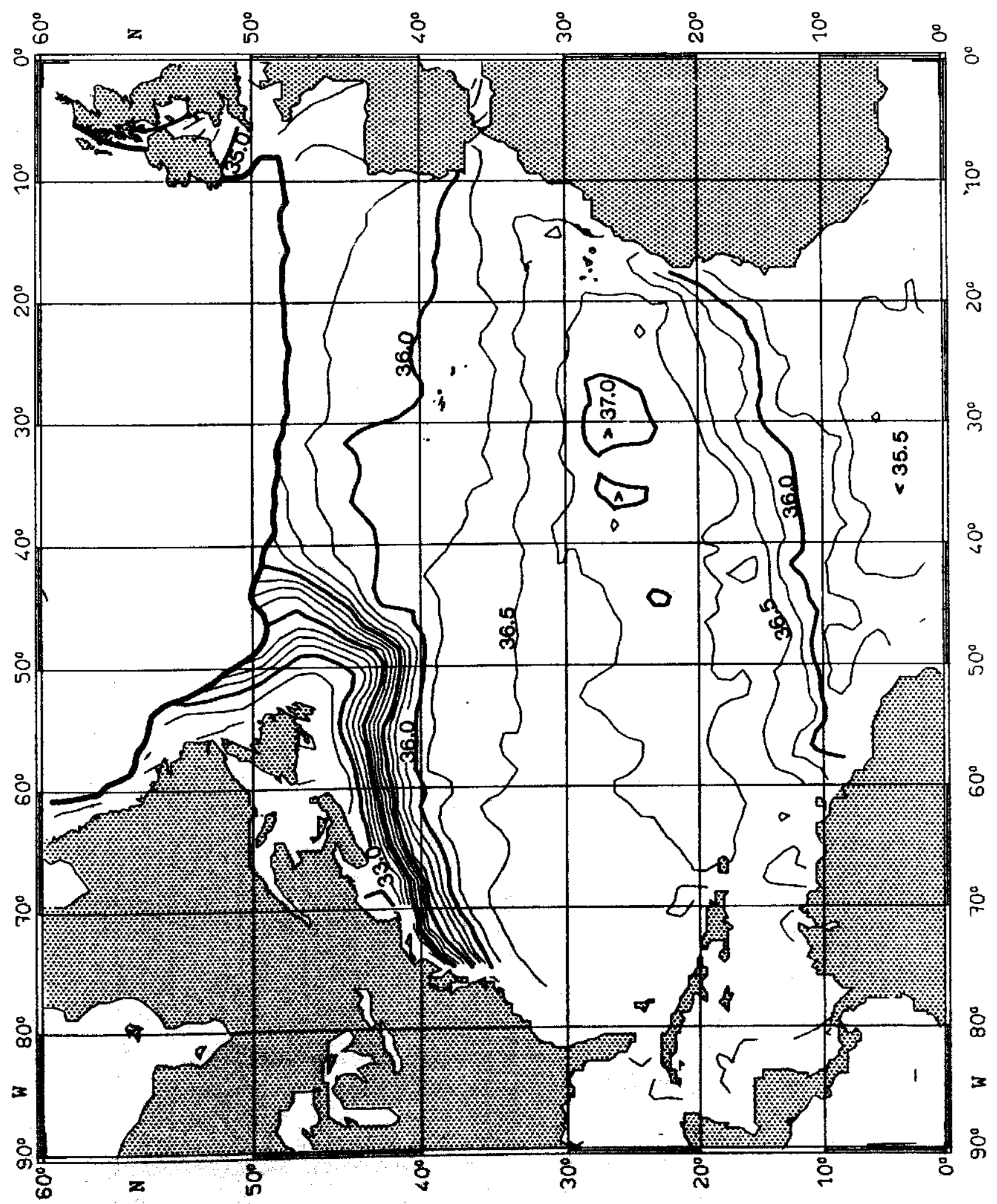


Fig. 106:

PRESSURE $\left(10^{4} \mathrm{~Pa}\right)$ on $\sigma_{\theta}=27.0 \mathrm{~kg} \mathrm{~m}^{-3}$ SEPTEMBER

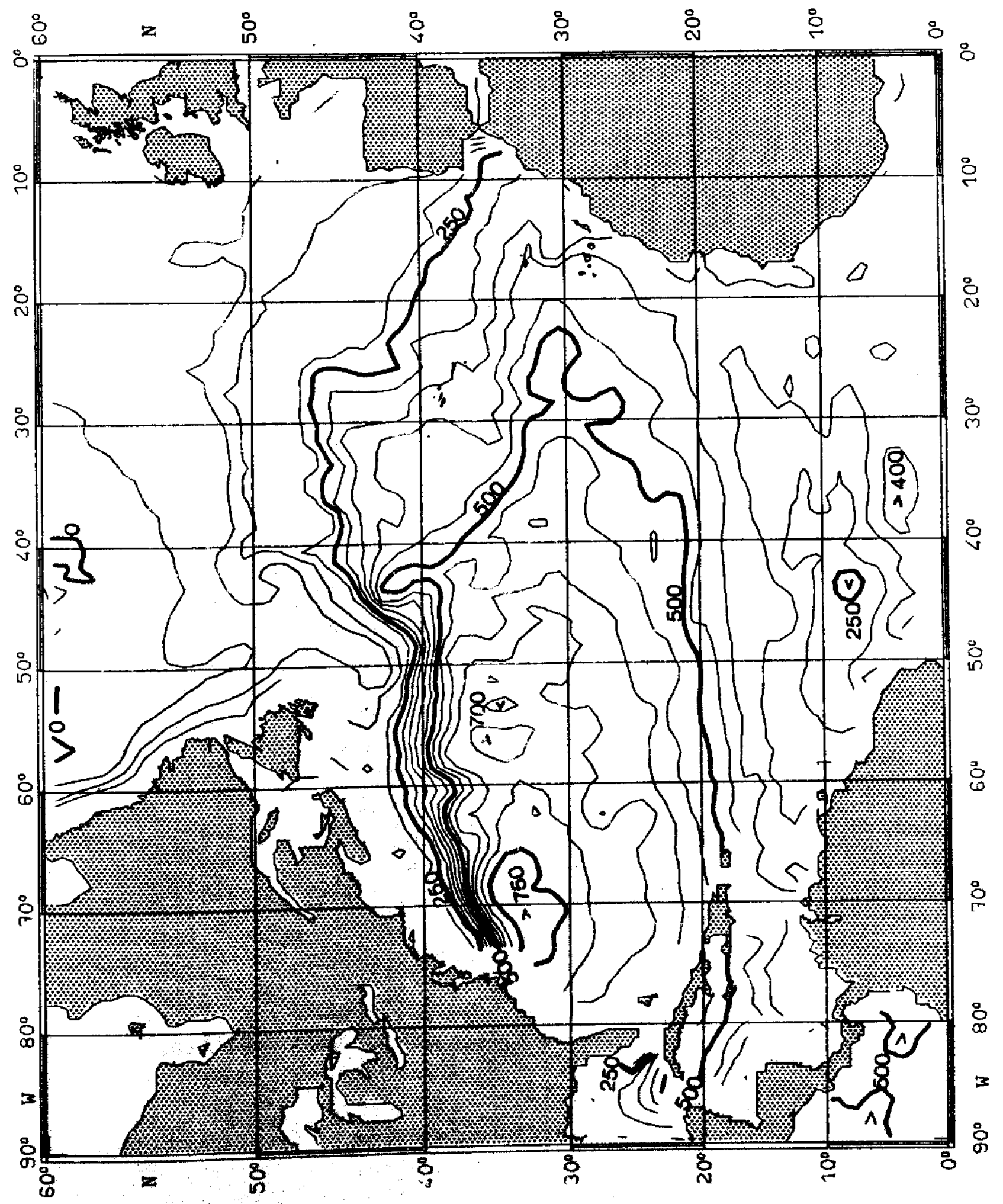


Fig. 107:

TEMPERATURE $\left({ }^{\circ} \mathrm{C}\right)$ on $\sigma_{\theta}=27.0 \mathrm{~kg} \mathrm{~m}^{-3} \quad$ SEPTEMBER

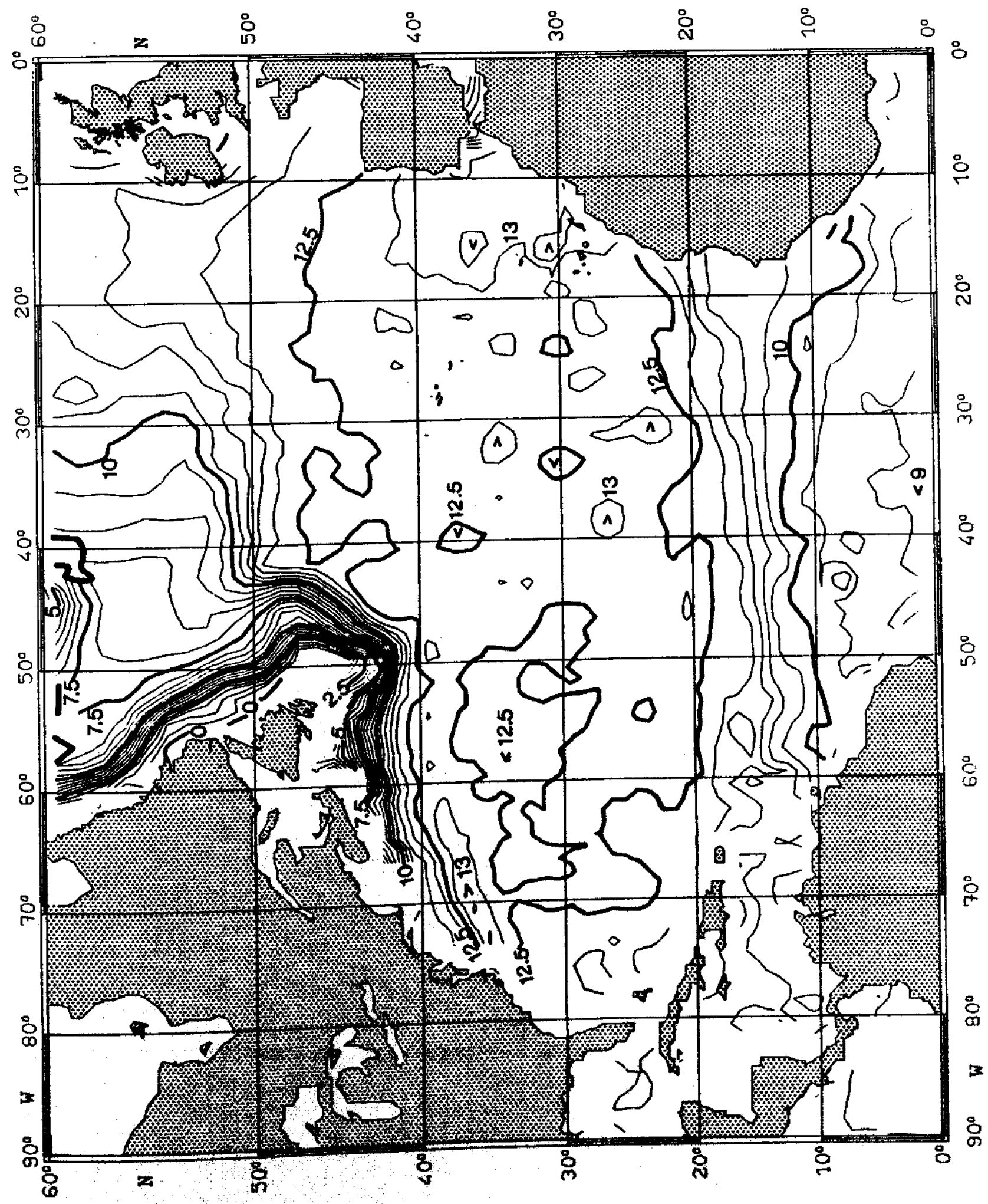


Fig. 108:

SALINITY $\left(10^{-3}\right)$ on $\sigma_{\theta}=27.0 \mathrm{~kg} \mathrm{~m}^{-3} \quad$ SEPTEMBER

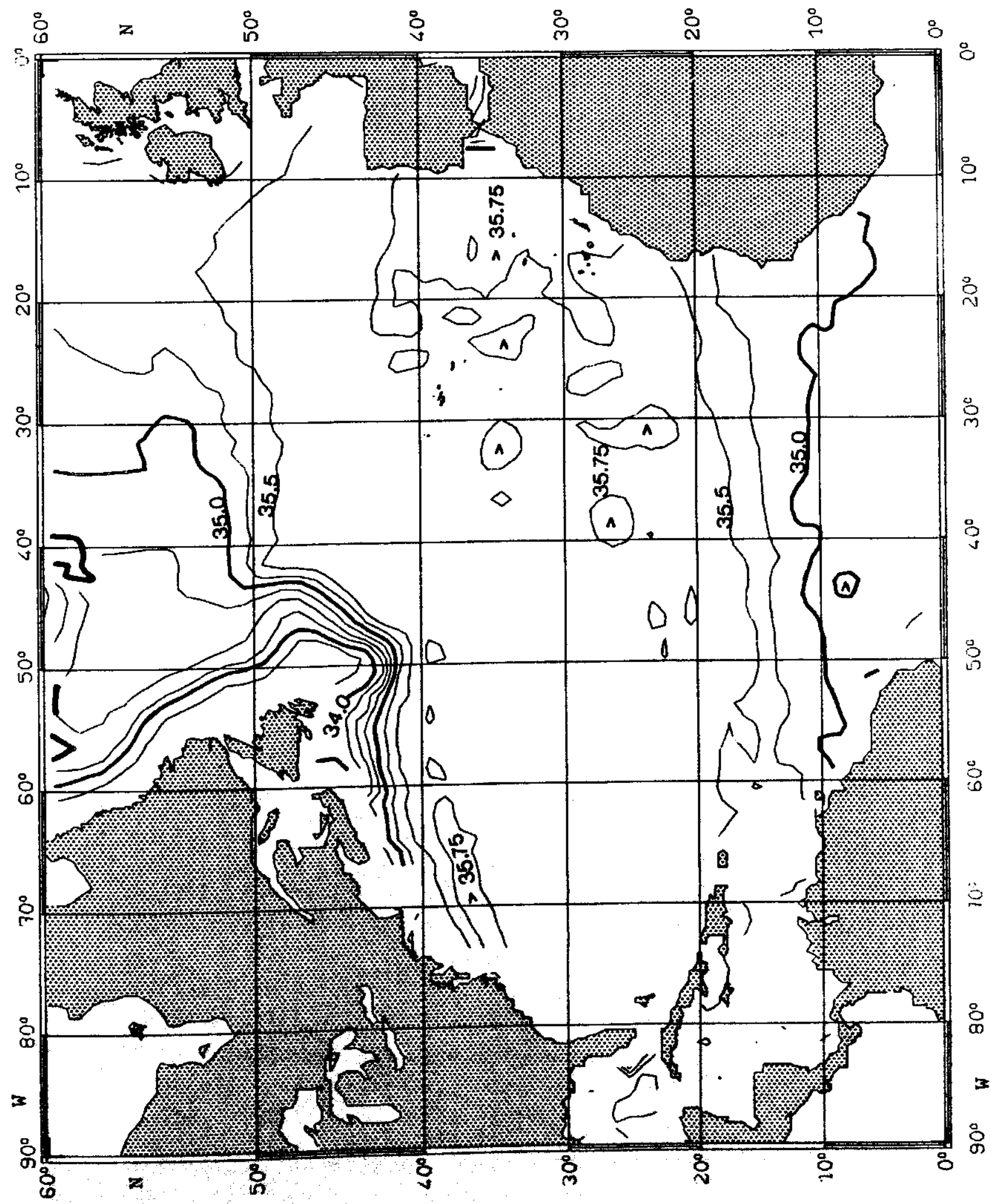


Fig. 109:

PRESSURE $\left(10^{4} \mathrm{~Pa}\right)$ on $\sigma_{\theta}=25.0 \mathrm{~kg} \mathrm{~m}^{-3} \quad$ OCTOBER

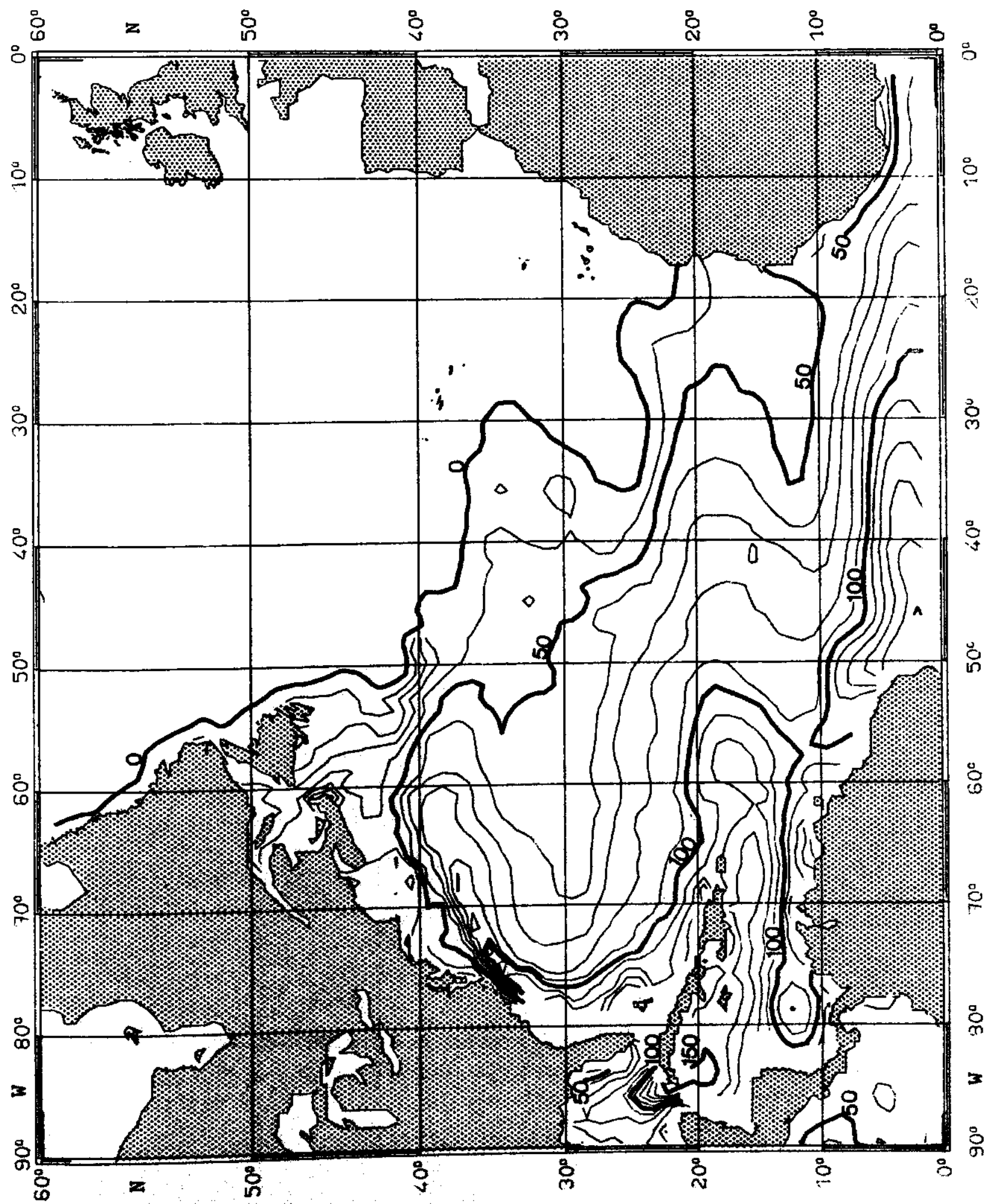


Fig. 110:

TEMPERATURE $\left({ }^{\circ} \mathrm{C}\right)$ on $\sigma_{\theta}=25.0 \mathrm{~kg} \mathrm{~m}^{-3} \quad$ OCTOBER

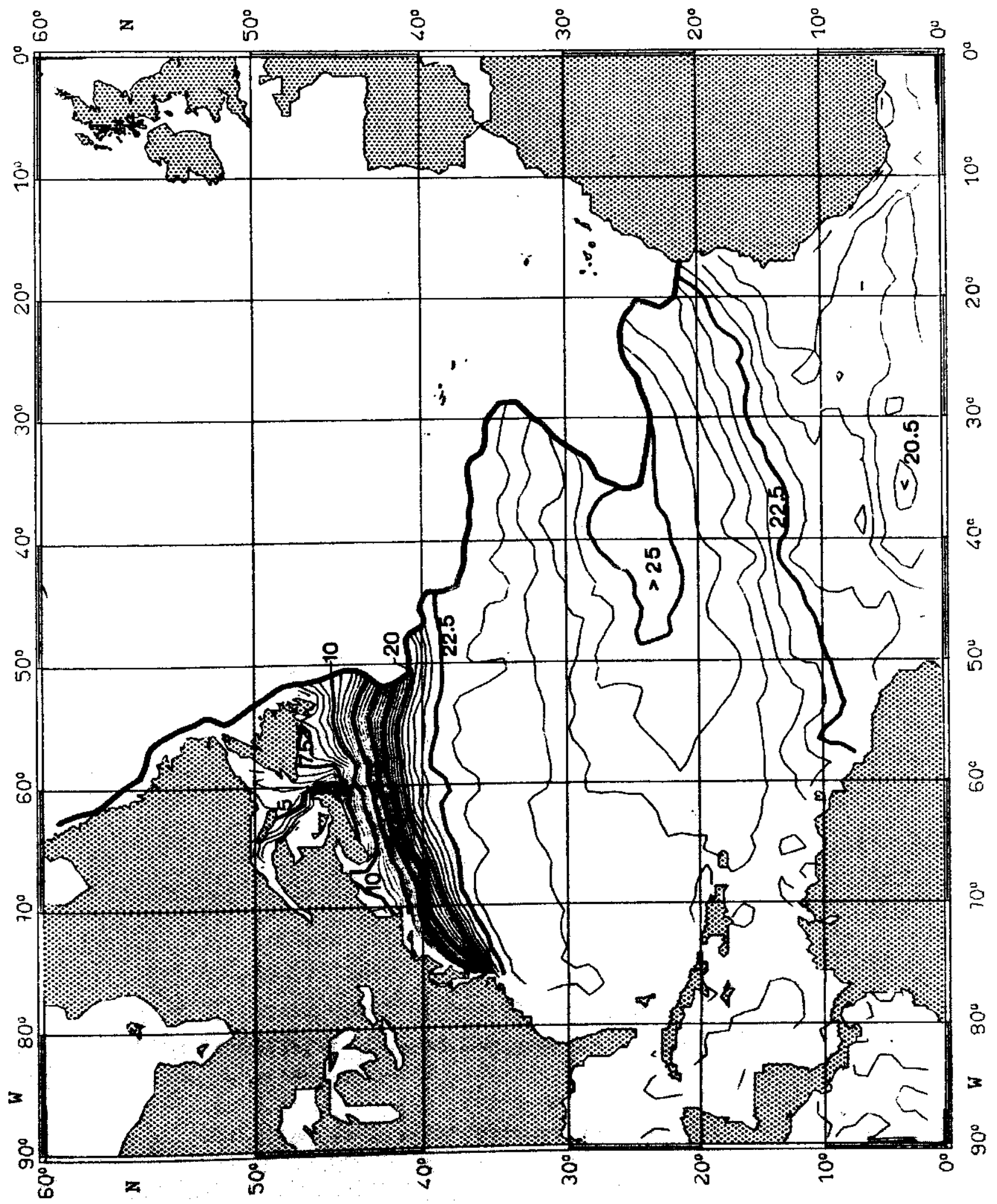


Fig. 111:

SALINITY $\left(10^{-3}\right)$ on $\sigma_{\theta}=25.0 \mathrm{~kg} \mathrm{~m}^{-3} \quad$ OCTOBER

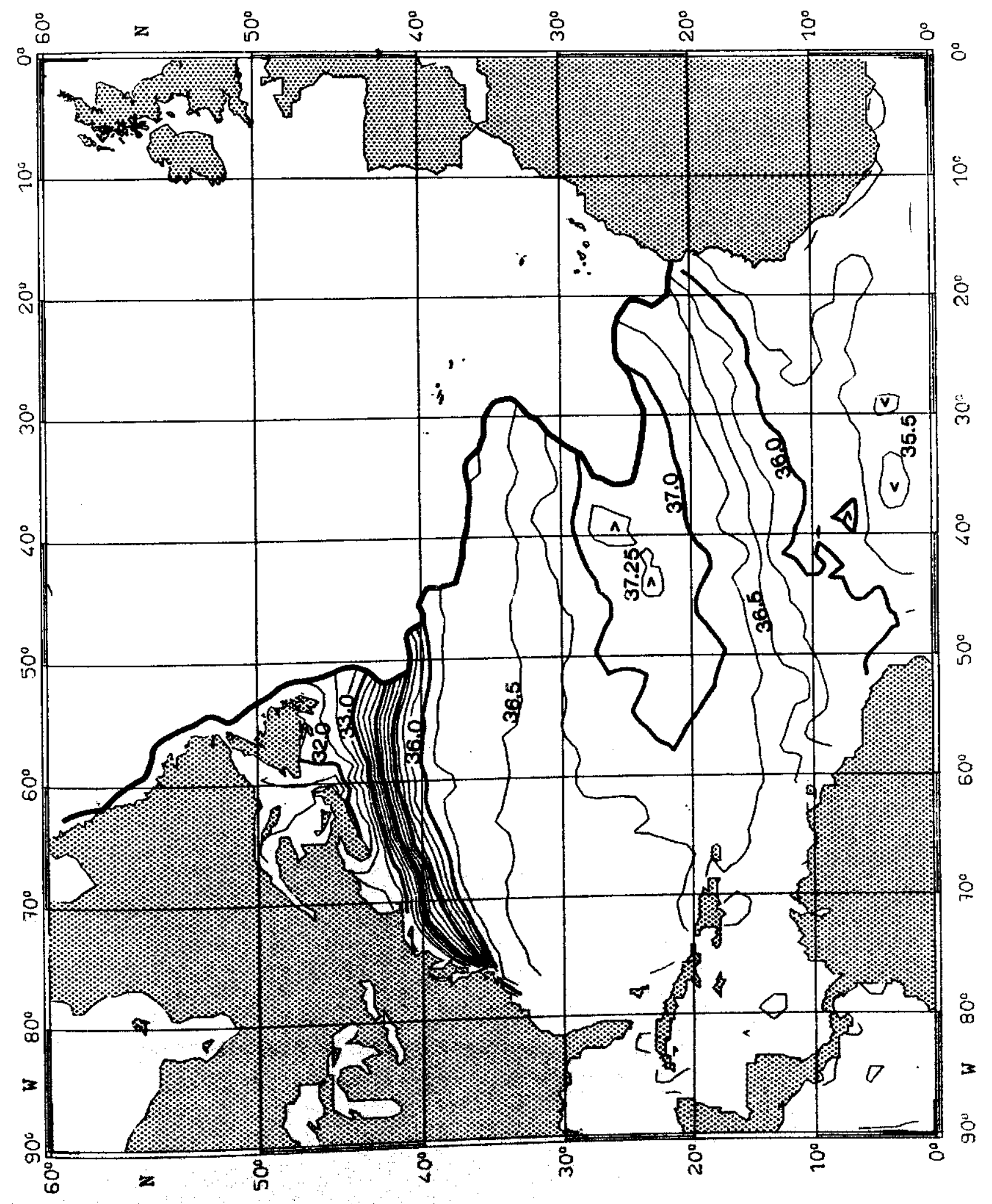


Fig. 112:

PRESSURE $\left(10^{4} \mathrm{~Pa}\right)$ on $\sigma_{\theta}=26.0 \mathrm{~kg} \mathrm{~m}^{-3} \quad$ OCTOBER

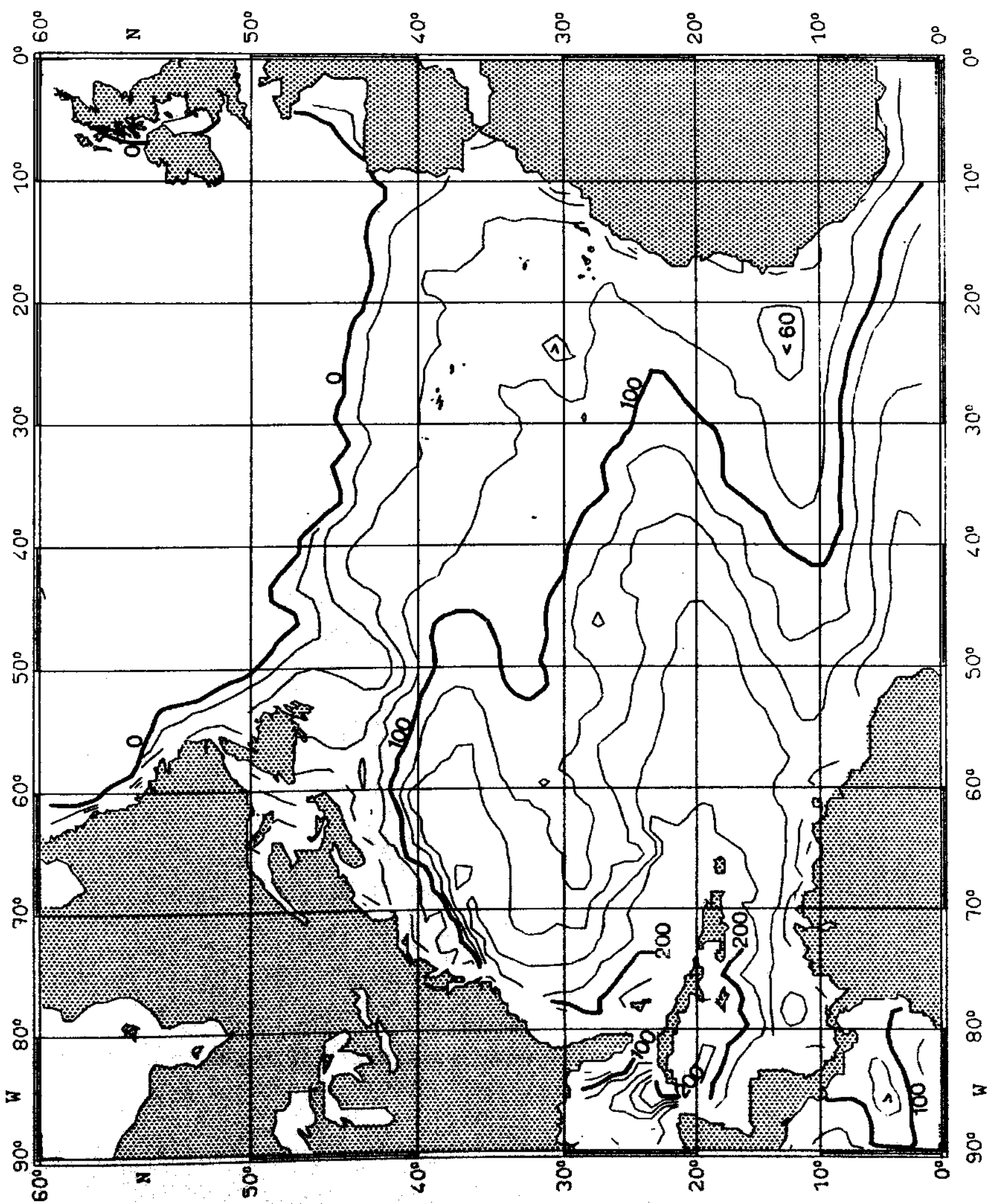


Fig. 113:

TEMPERATURE $\left({ }^{\circ} \mathrm{C}\right)$ on $\sigma_{\theta}=26.0 \mathrm{~kg} \mathrm{~m}^{-3} \quad$ OCTOBER

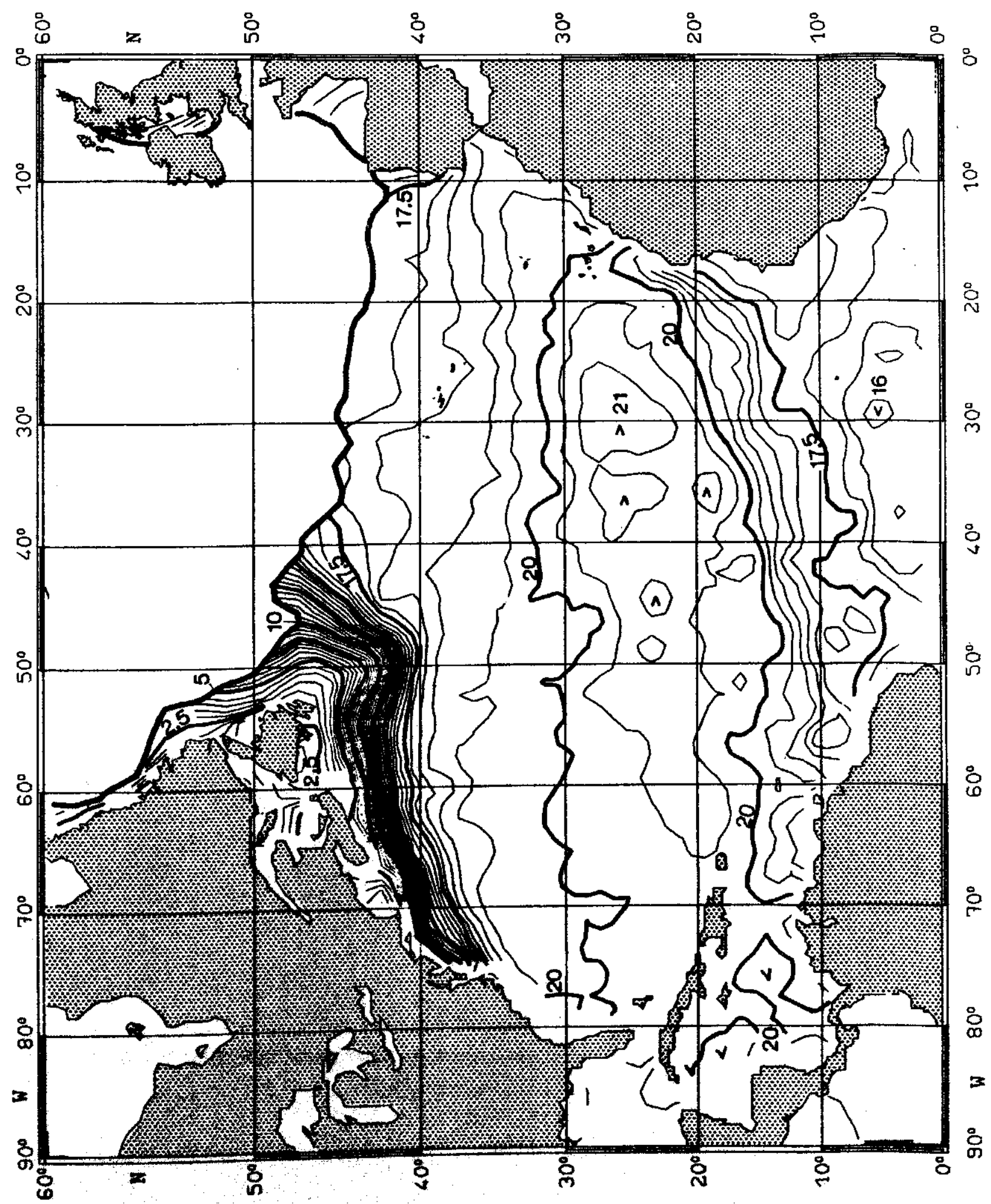


Fig. 114:

SALINITY $\left(10^{-3}\right)$ on $\sigma_{\theta}=26.0 \mathrm{~kg} \mathrm{~m}^{-3} \quad$ OCTOBER

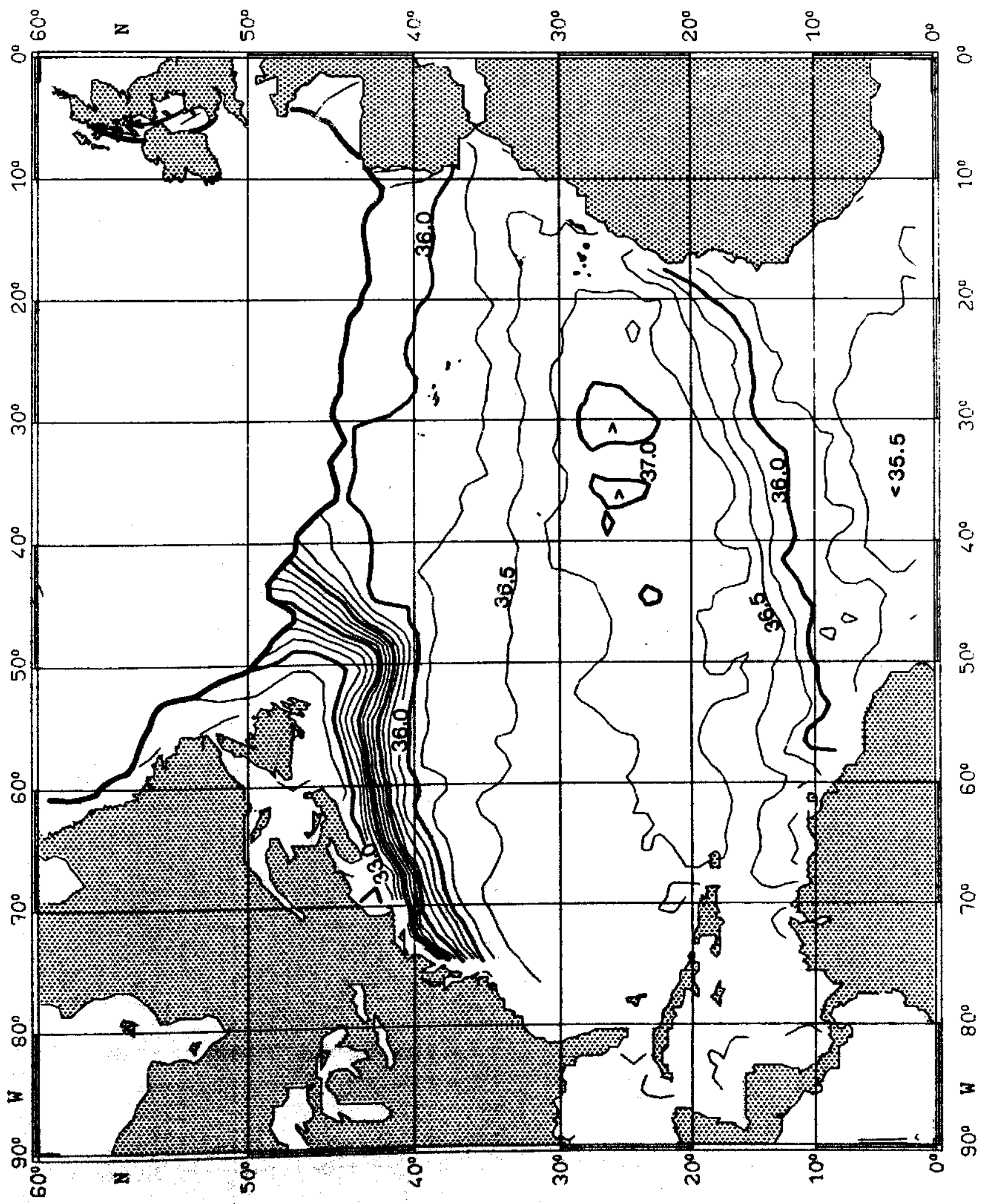


Fig. 115:

PRESSURE $\left(10^{4} \mathrm{~Pa}\right)$ on $\sigma_{\theta}=27.0 \mathrm{~kg} \mathrm{~m}^{-3} \quad$ OCTOBER

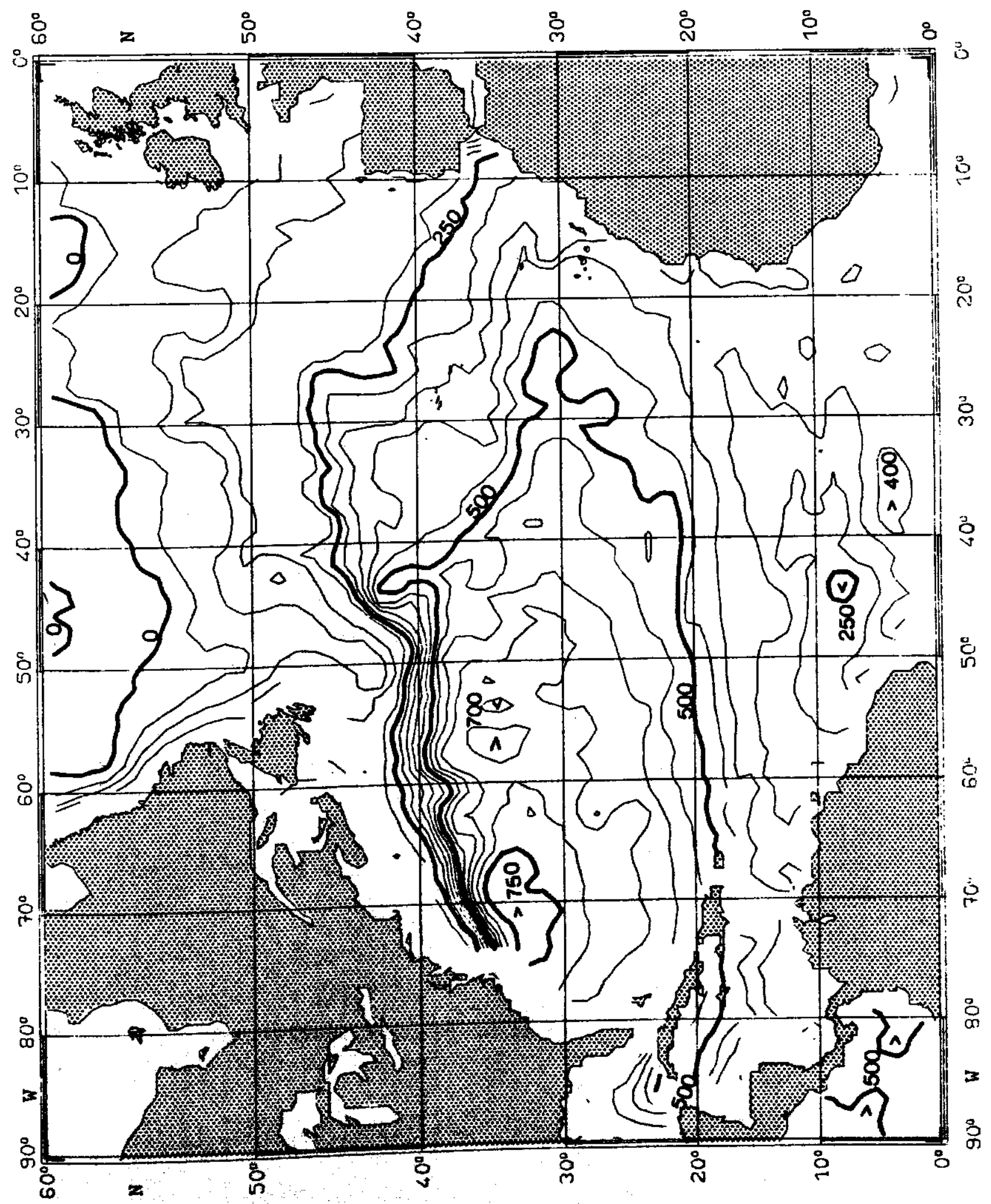


Fig. 116:

TEMPERATURE $\left({ }^{\circ} \mathrm{C}\right)$ on $\sigma_{\theta}=27.0 \mathrm{~kg} \mathrm{~m}^{-3} \quad$ OCTOBER

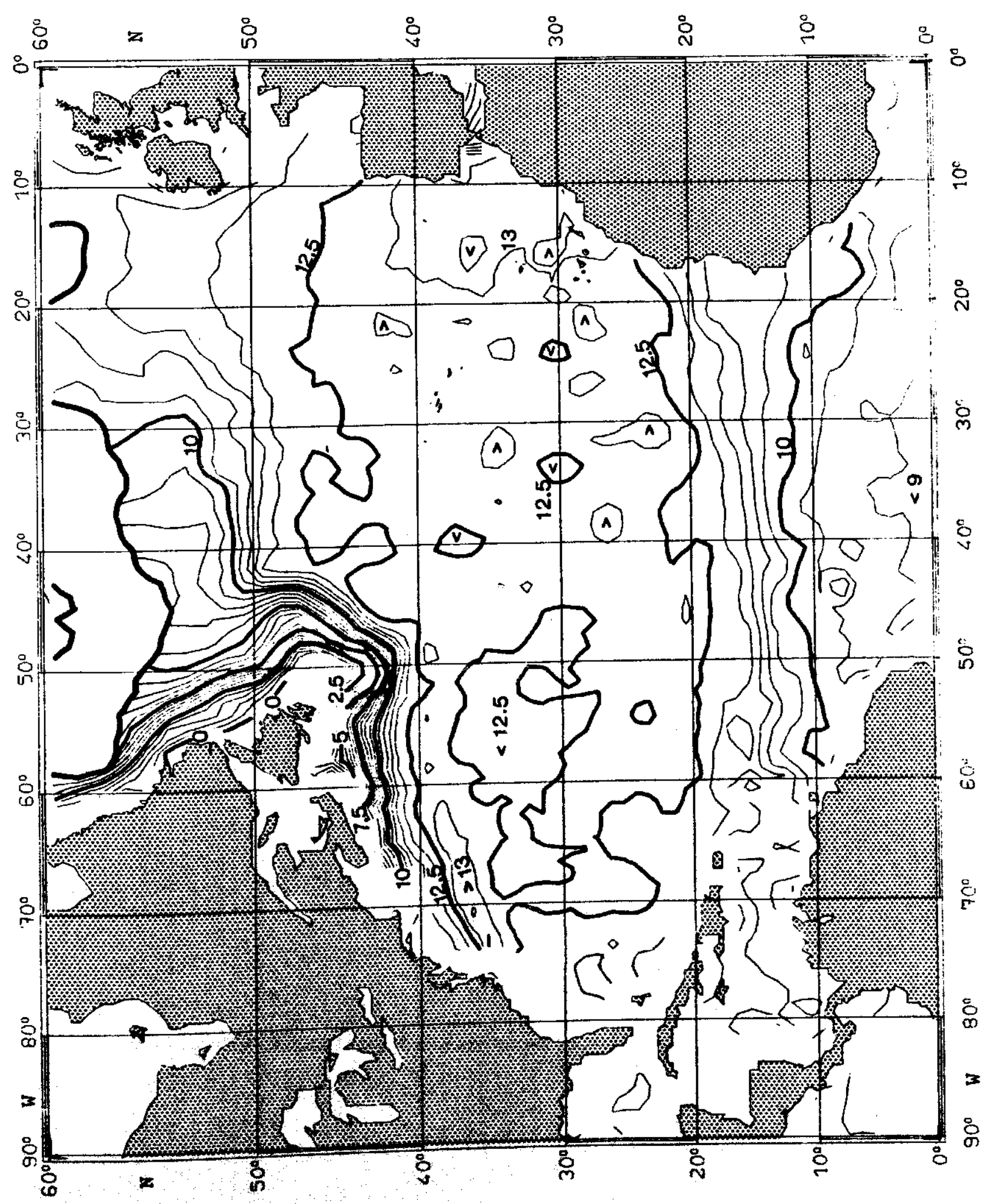


Fig. 117:

SALINITY $\left(10^{-3}\right)$ on $\sigma_{\theta}=27.0 \mathrm{~kg} \mathrm{~m}^{-3} \quad$ OCTOBER

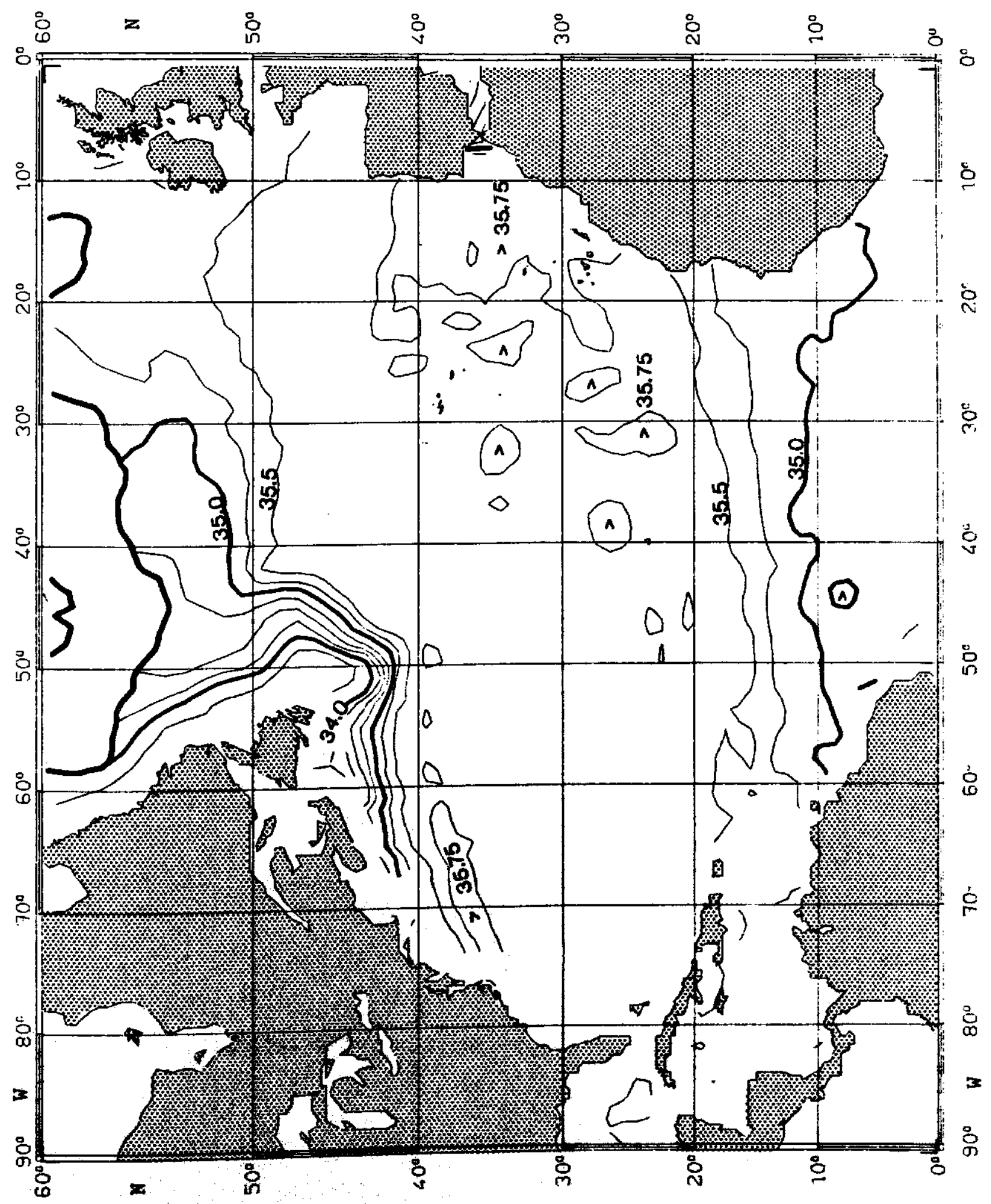


Fig. 118:

PRESSURE $\left(10^{4} \mathrm{~Pa}\right)$ on $\sigma_{\theta}=25.0 \mathrm{~kg} \mathrm{~m}^{-3} \quad$ NOVEMBER

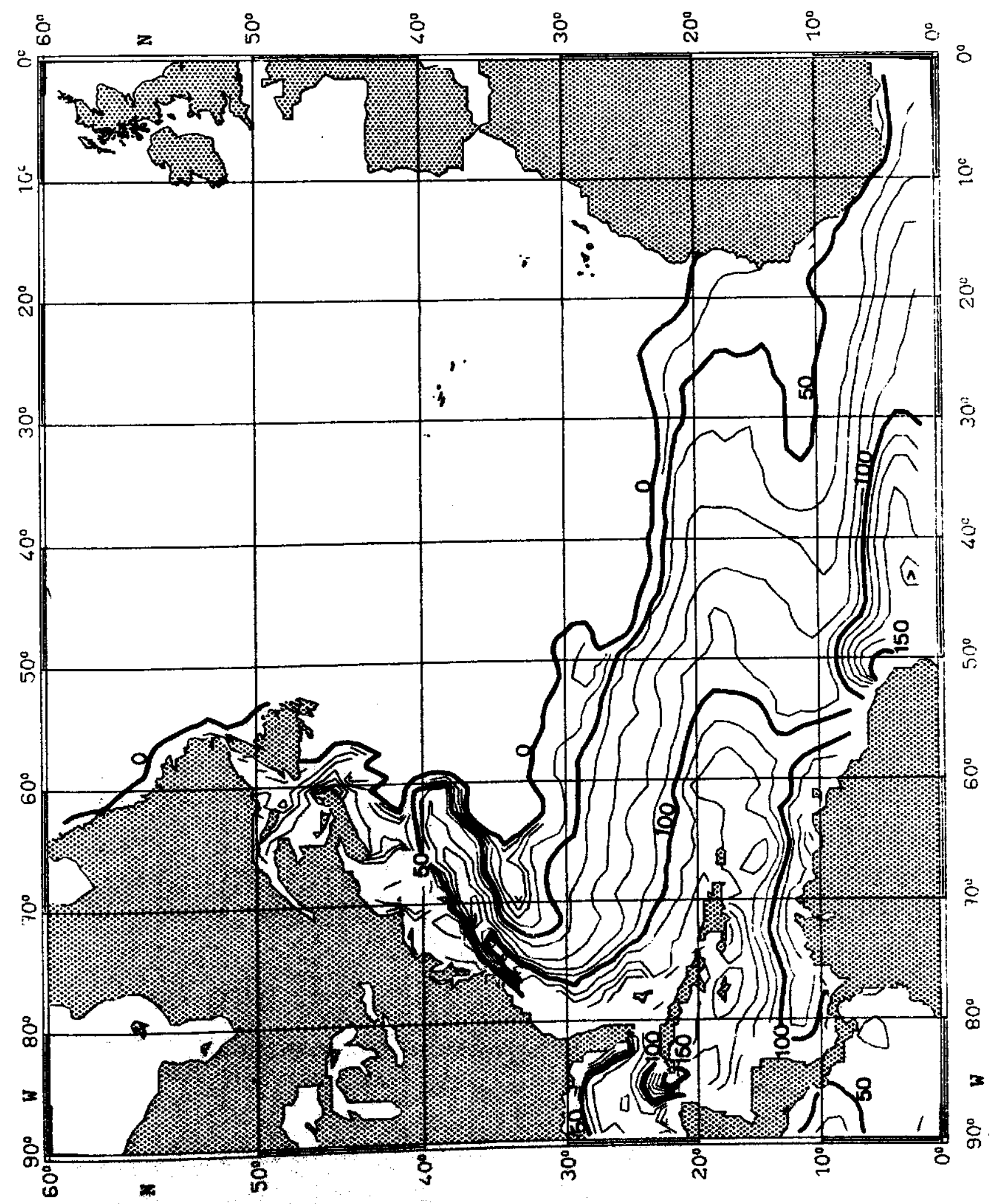


Fig. 119:

TEMPERATURE $\left({ }^{\circ} \mathrm{C}\right)$ on $\sigma_{\theta}=25.0 \mathrm{~kg} \mathrm{~m}^{-3} \quad$ NOVEMBER

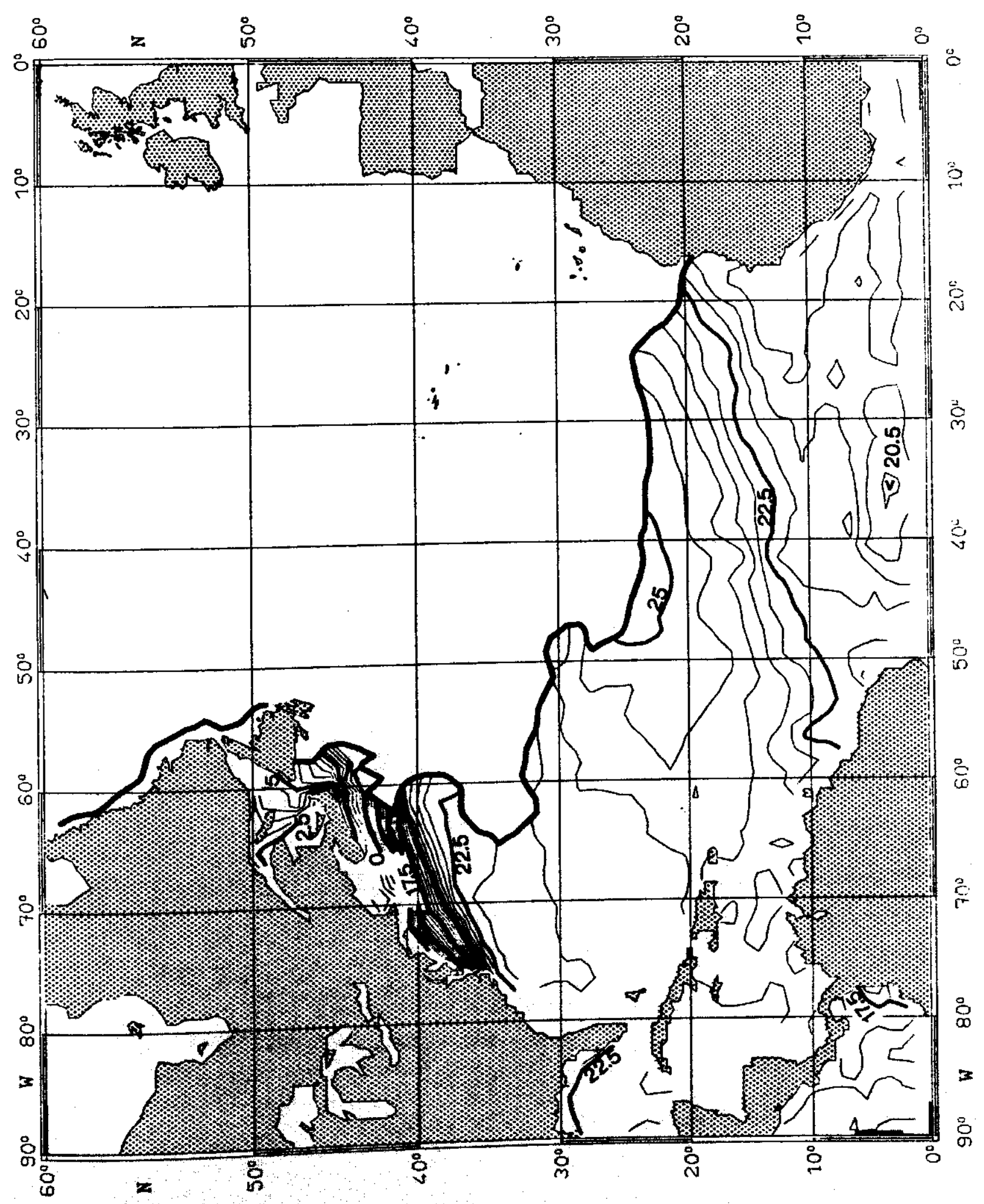


Fig. 120:

SALINITY $\left(10^{-3}\right)$ on $\sigma_{\theta}=25.0 \mathrm{~kg} \mathrm{~m}^{-3} \quad$ NOVEMBER

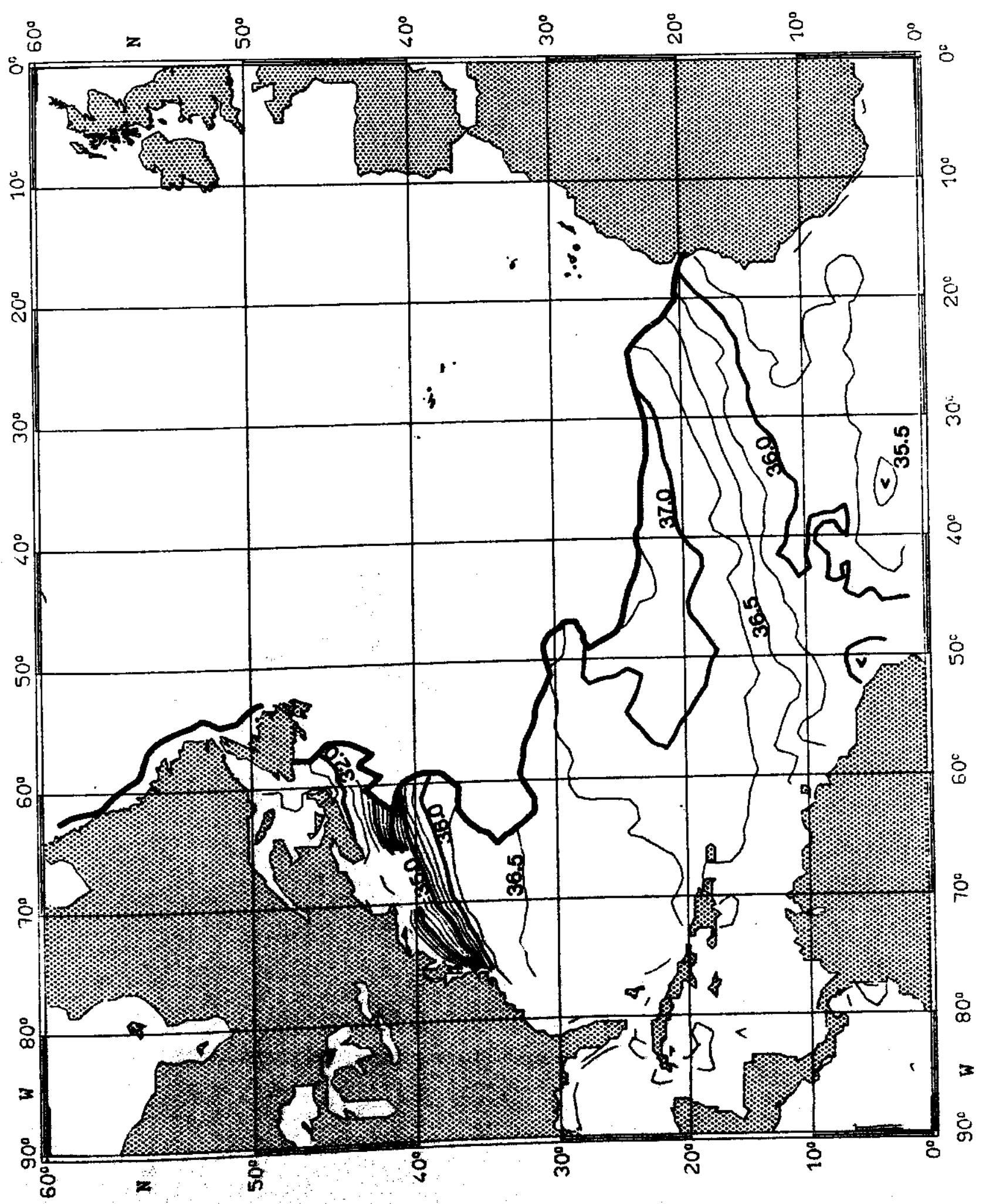


Fig. 121:

PRESSURE $\left(10^{4} \mathrm{~Pa}\right)$ on $\sigma_{\theta}=26.0 \mathrm{~kg} \mathrm{~m}^{-3} \quad$ NOVEMBER

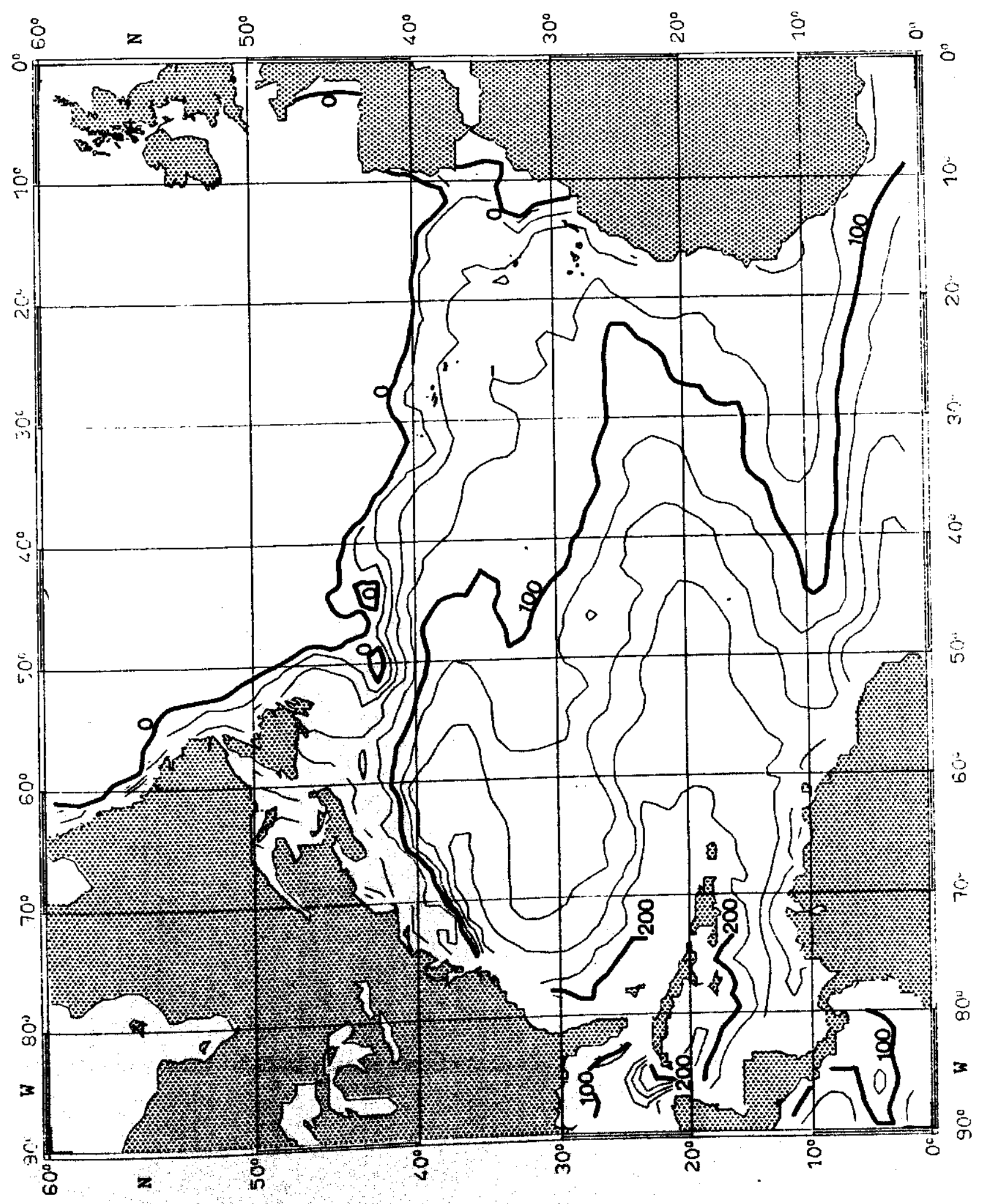


Fig. 122:

TEMPERATURE $\left({ }^{\circ} \mathrm{C}\right)$ on $\sigma_{\theta}=26.0 \mathrm{~kg} \mathrm{~m}^{-3} \quad$ NOVEMBER

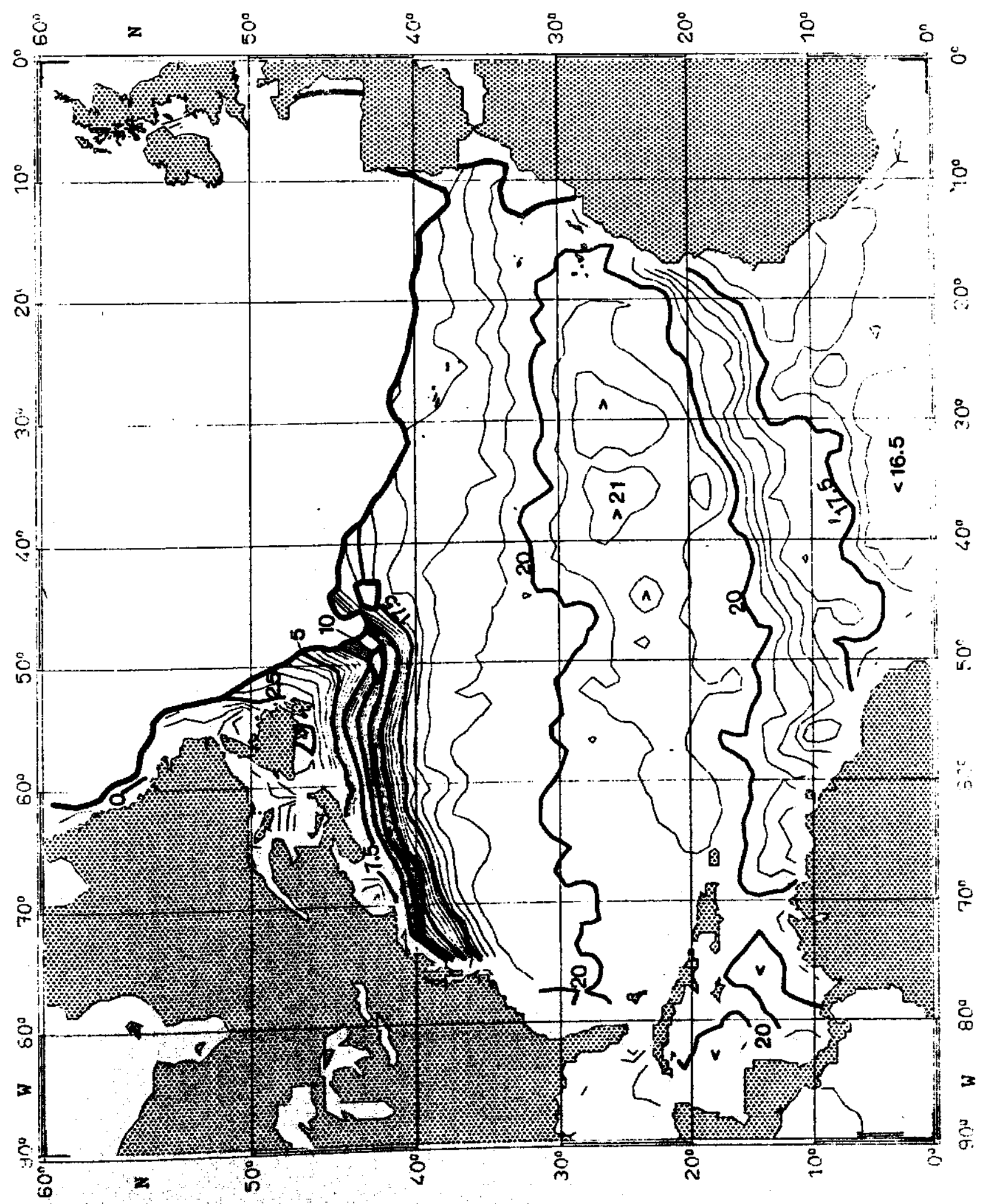


Fig. 123:

SALINITY $\left(10^{-3}\right)$ on $\sigma_{\theta}=26.0 \mathrm{~kg} \mathrm{~m}^{-3} \quad$ NOVEMBER

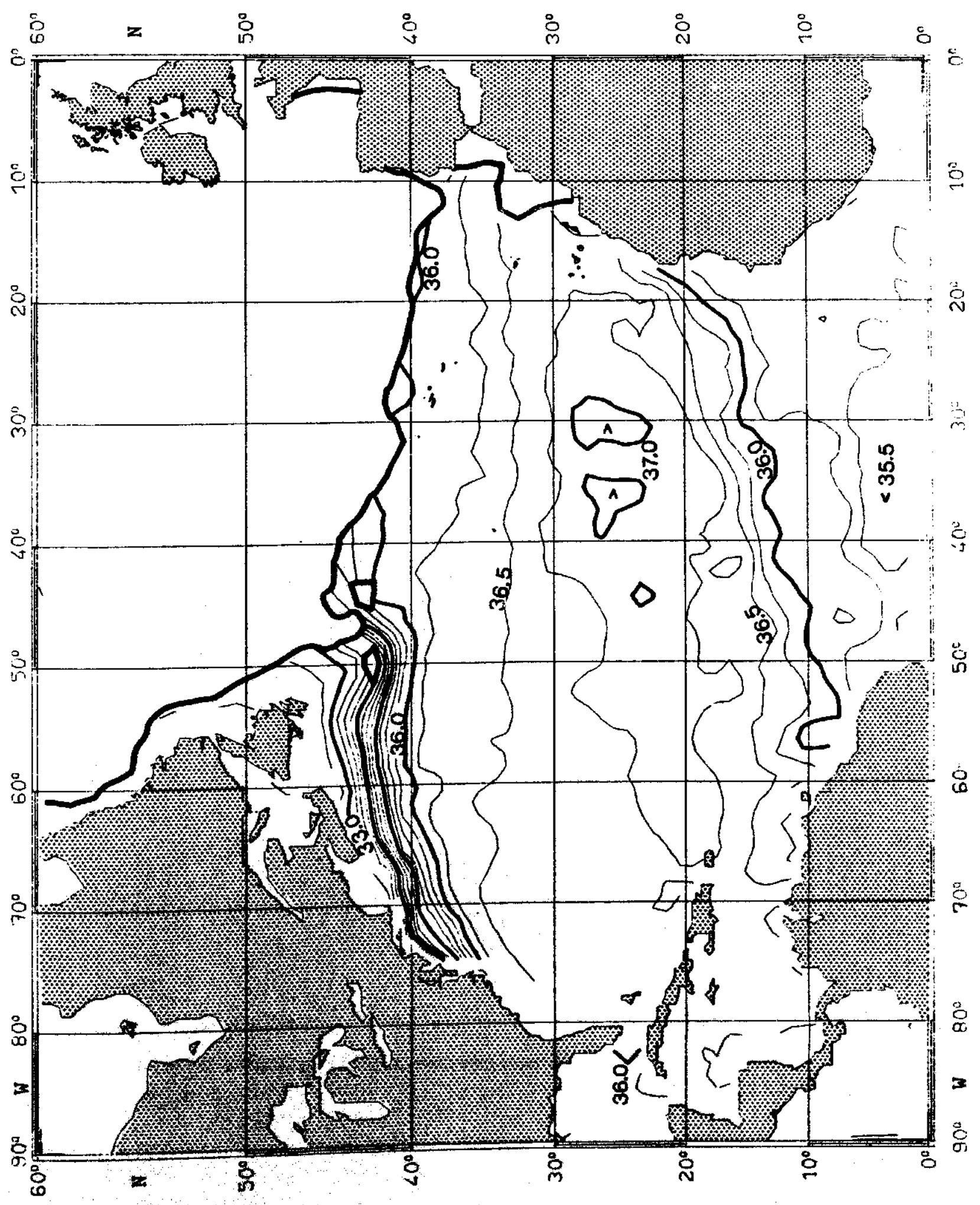


Fig. 124:

PRESSURE $\left(10^{4} \mathrm{~Pa}\right)$ on $\sigma_{\theta}=27.0 \mathrm{~kg} \mathrm{~m}^{-3} \quad$ NOVEMBER

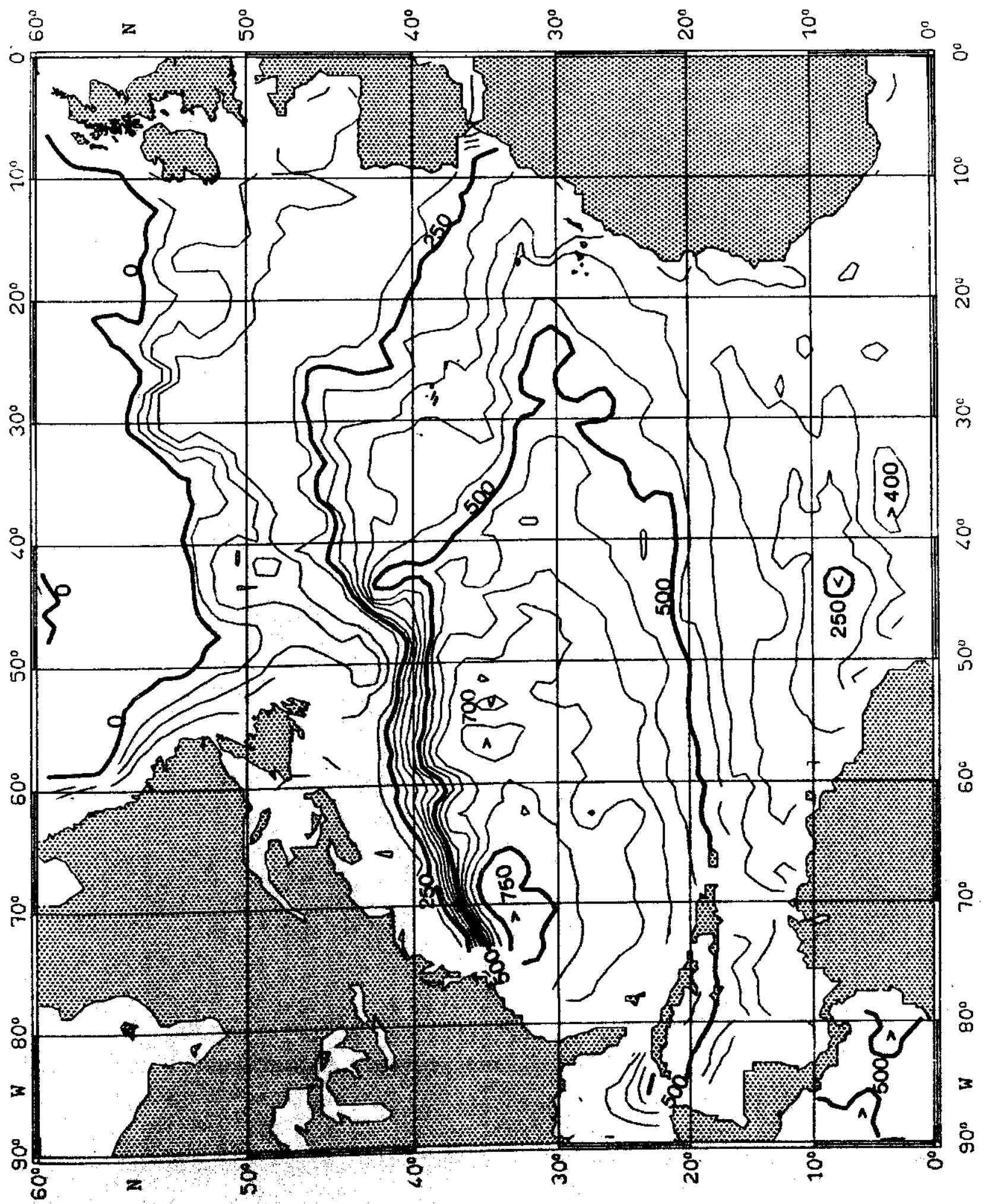


Fig. 125:

TEMPERATURE $\left({ }^{\circ} \mathrm{C}\right)$ on $\sigma_{\theta}=27.0 \mathrm{~kg} \mathrm{~m}^{-3} \quad$ NOVEMBER

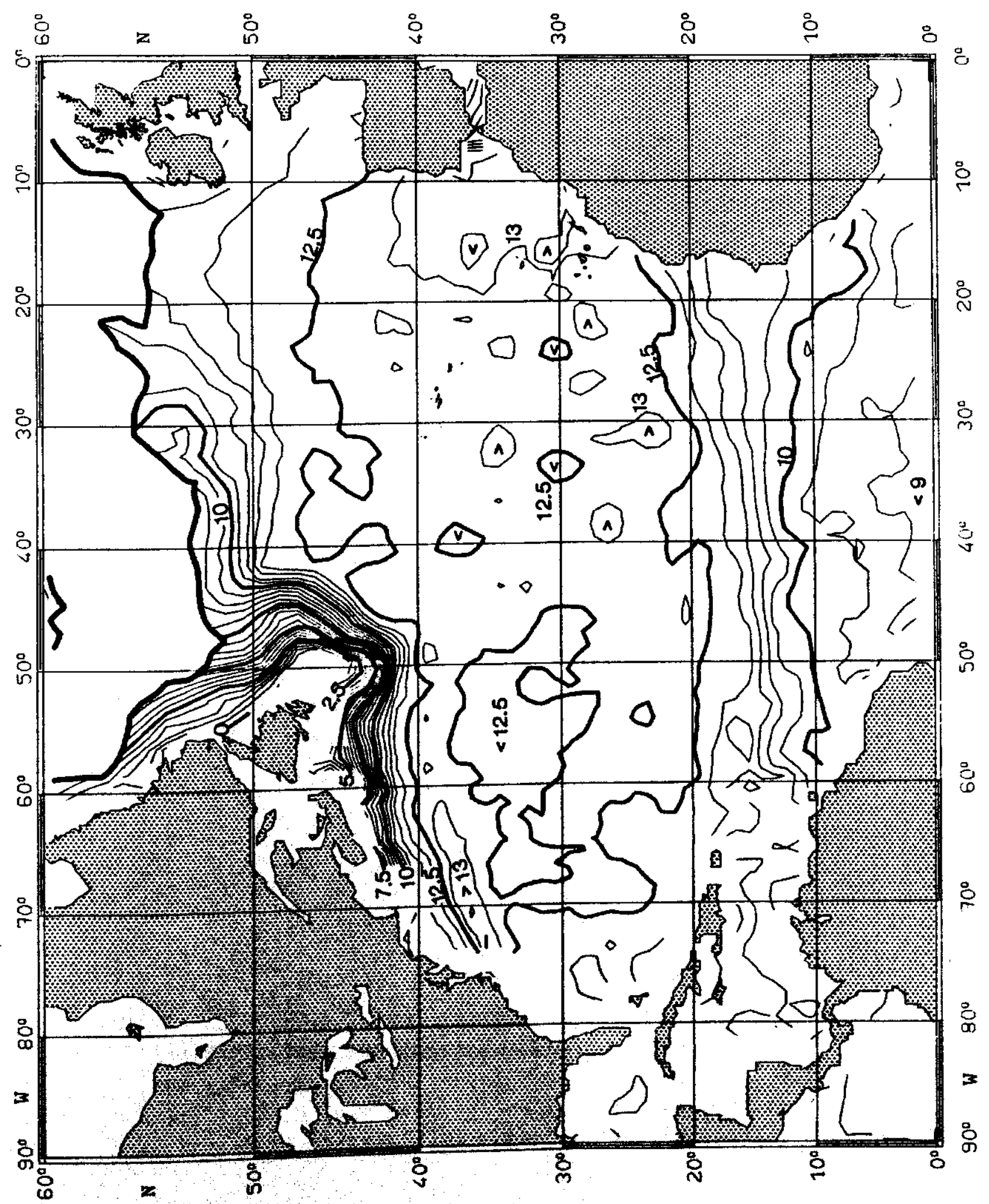


Fig. 126:

SALINITY $\left(10^{-3}\right)$ on $\sigma_{\theta}=27.0 \mathrm{~kg} \mathrm{~m}^{-3} \quad$ NOVEMBER

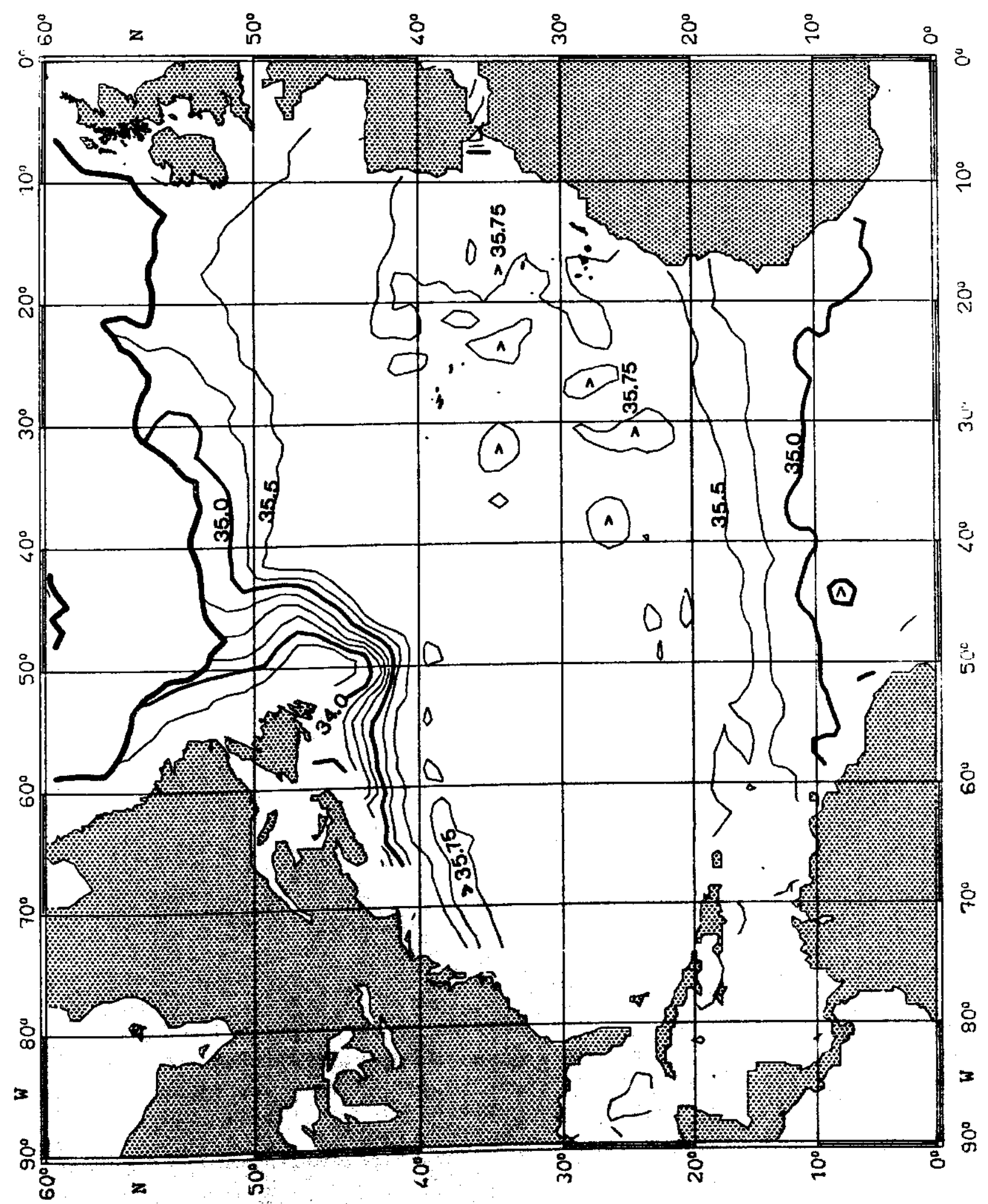


Fig. 127:

PRESSURE $\left(10^{4} \mathrm{~Pa}\right)$ on $\delta_{\theta}=25.0 \mathrm{~kg} \mathrm{~m}^{-3}$ DECEMBER

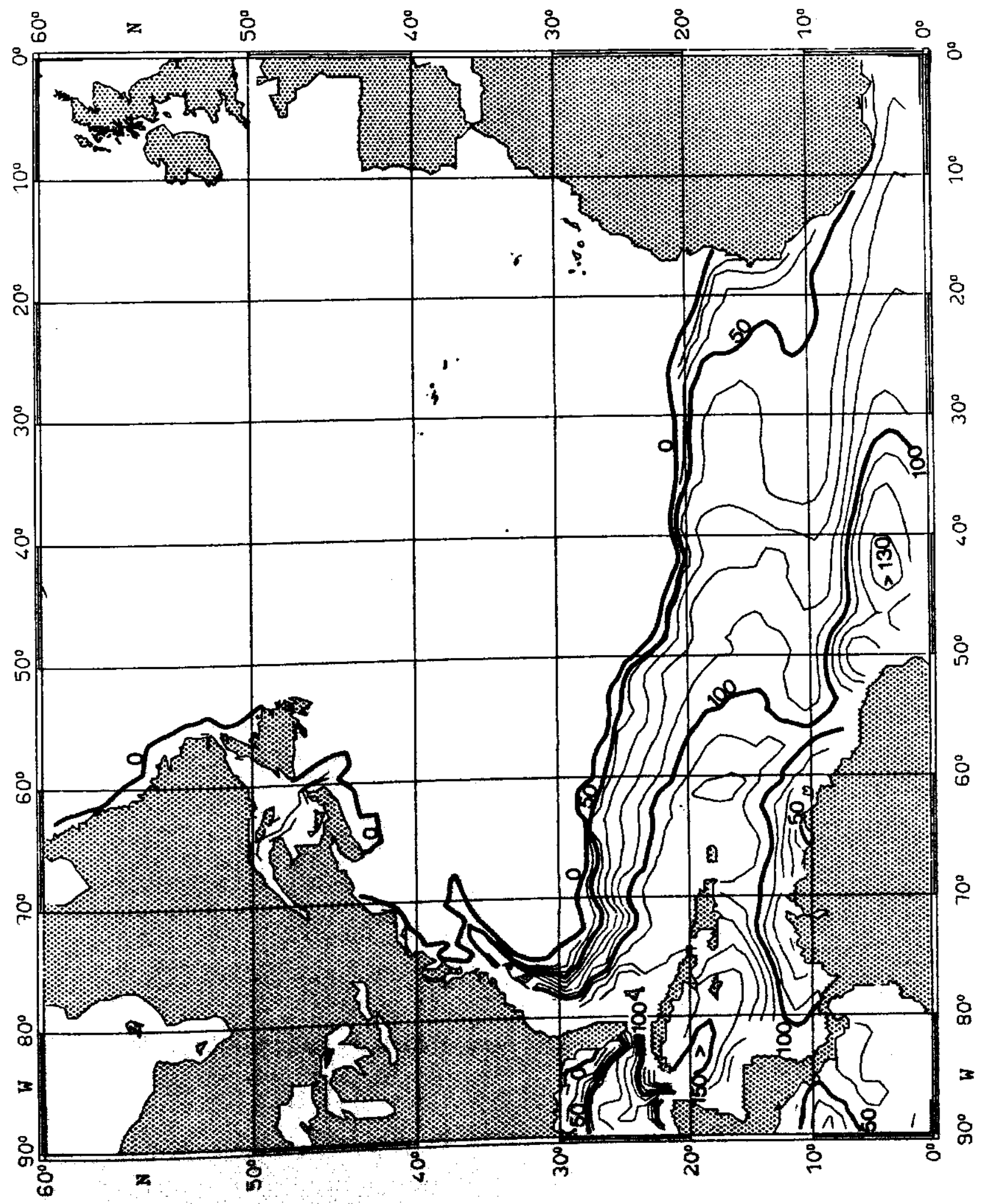


Fig. 128:

TEMPERATURE $\left({ }^{\circ} \mathrm{C}\right)$ on $\sigma_{\theta}=25.0 \mathrm{~kg} \mathrm{~m}^{-3}$ DECEMBER

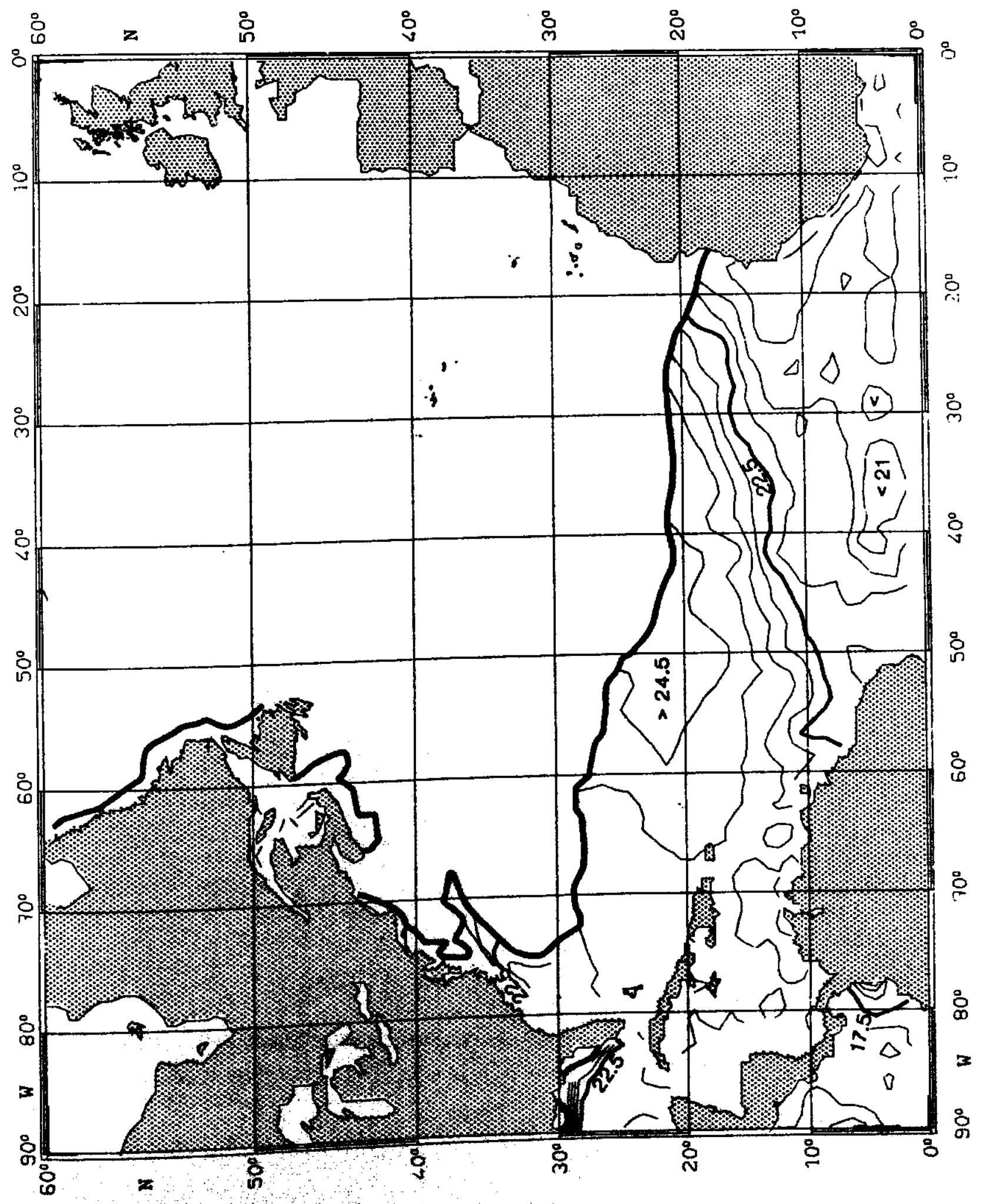


Fig. 129:

SALINITY $\left(10^{-3}\right)$ on $\sigma_{\theta}=25.0 \mathrm{~kg} \mathrm{~m}^{-3} \quad$ DECEMBER

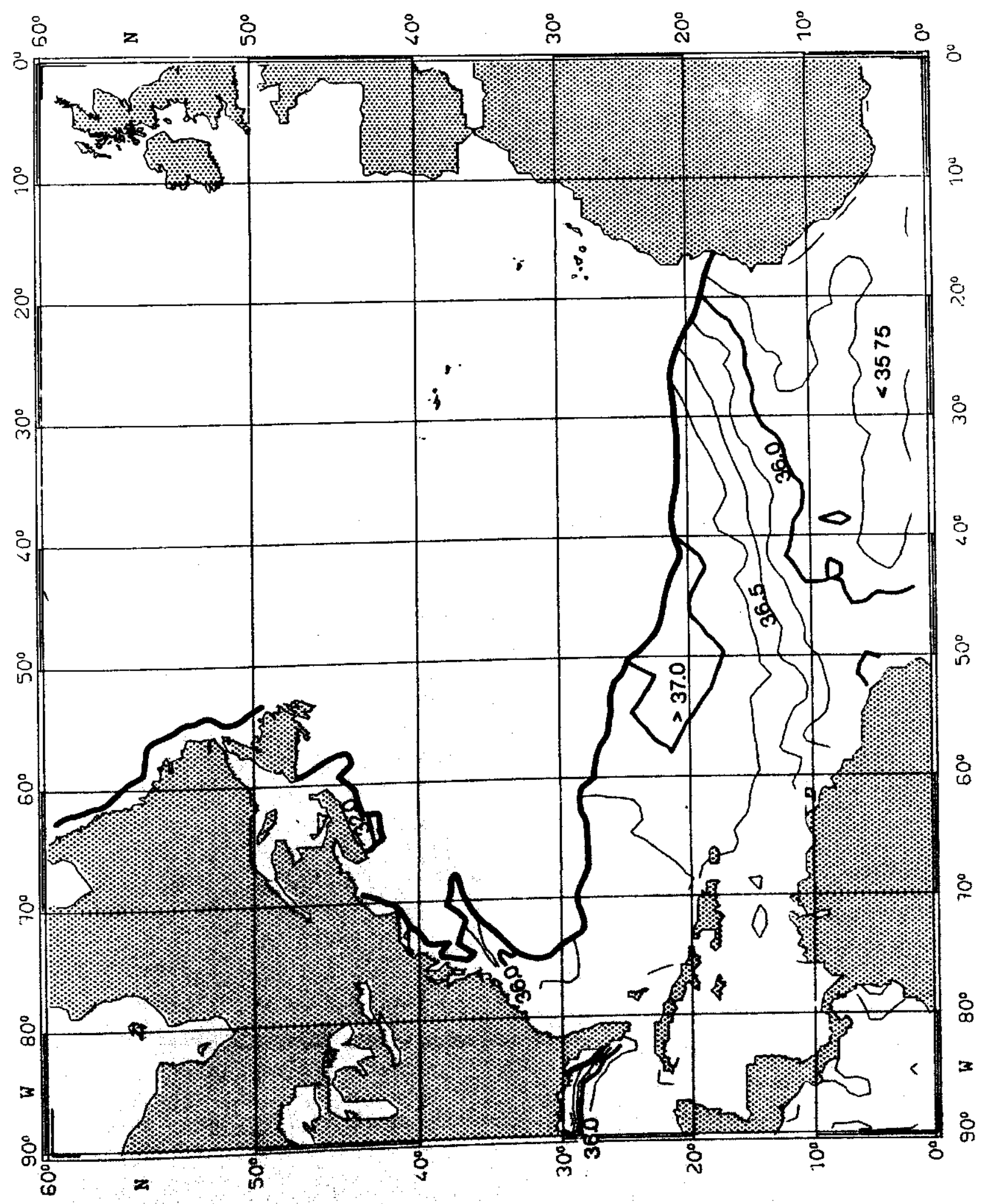


Fig. 130:

PRESSURE $\left(10^{4} \mathrm{~Pa}\right)$ on $\sigma_{\theta}=26.0 \mathrm{~kg} \mathrm{~m}^{-3}$ DECEMBER

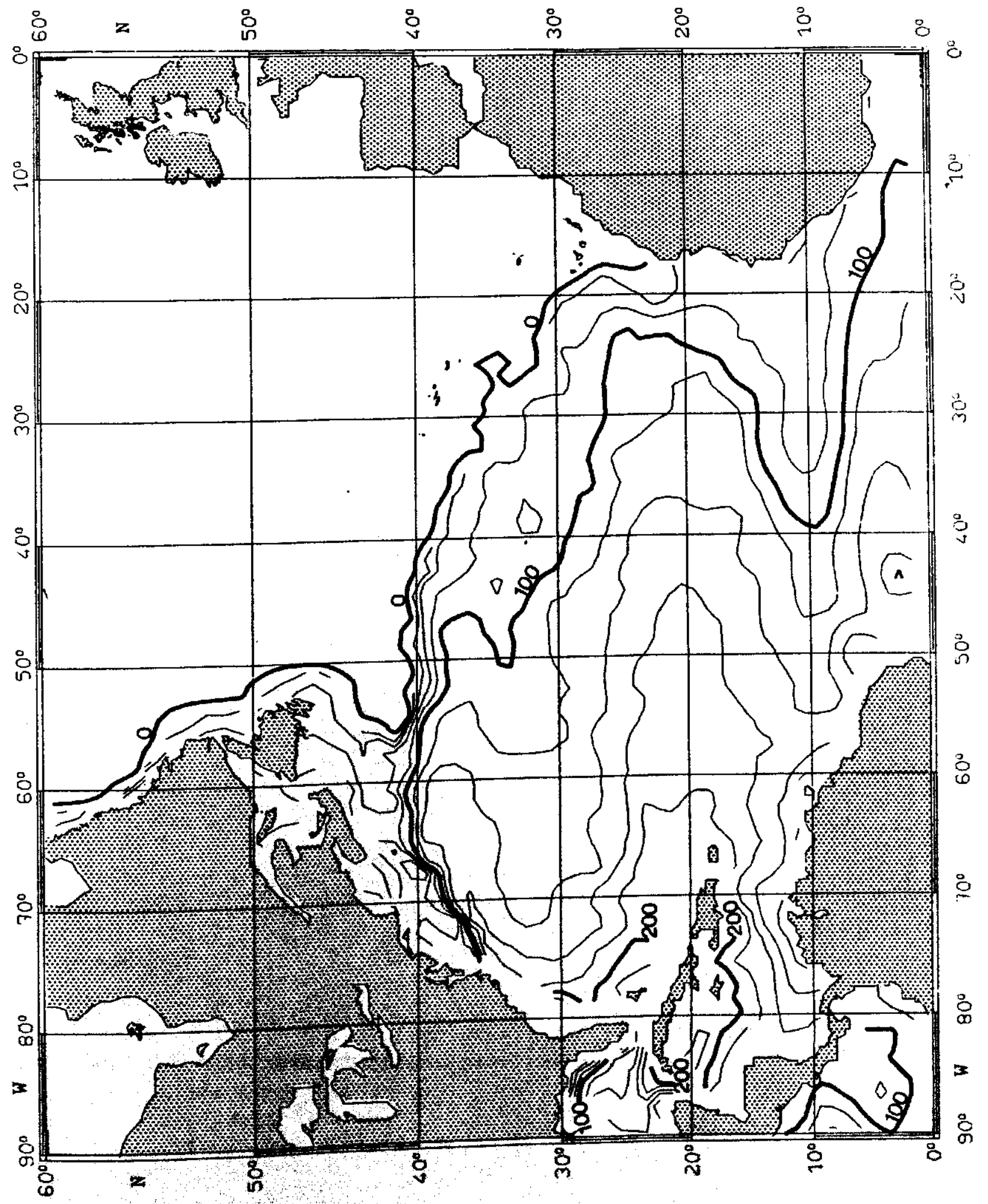


Fig. 131:

TEMPERATURE $\left({ }^{\circ} \mathrm{C}\right)$ on $\sigma_{\theta}=26.0 \mathrm{~kg} \mathrm{~m}^{-3}$ DECEMBER

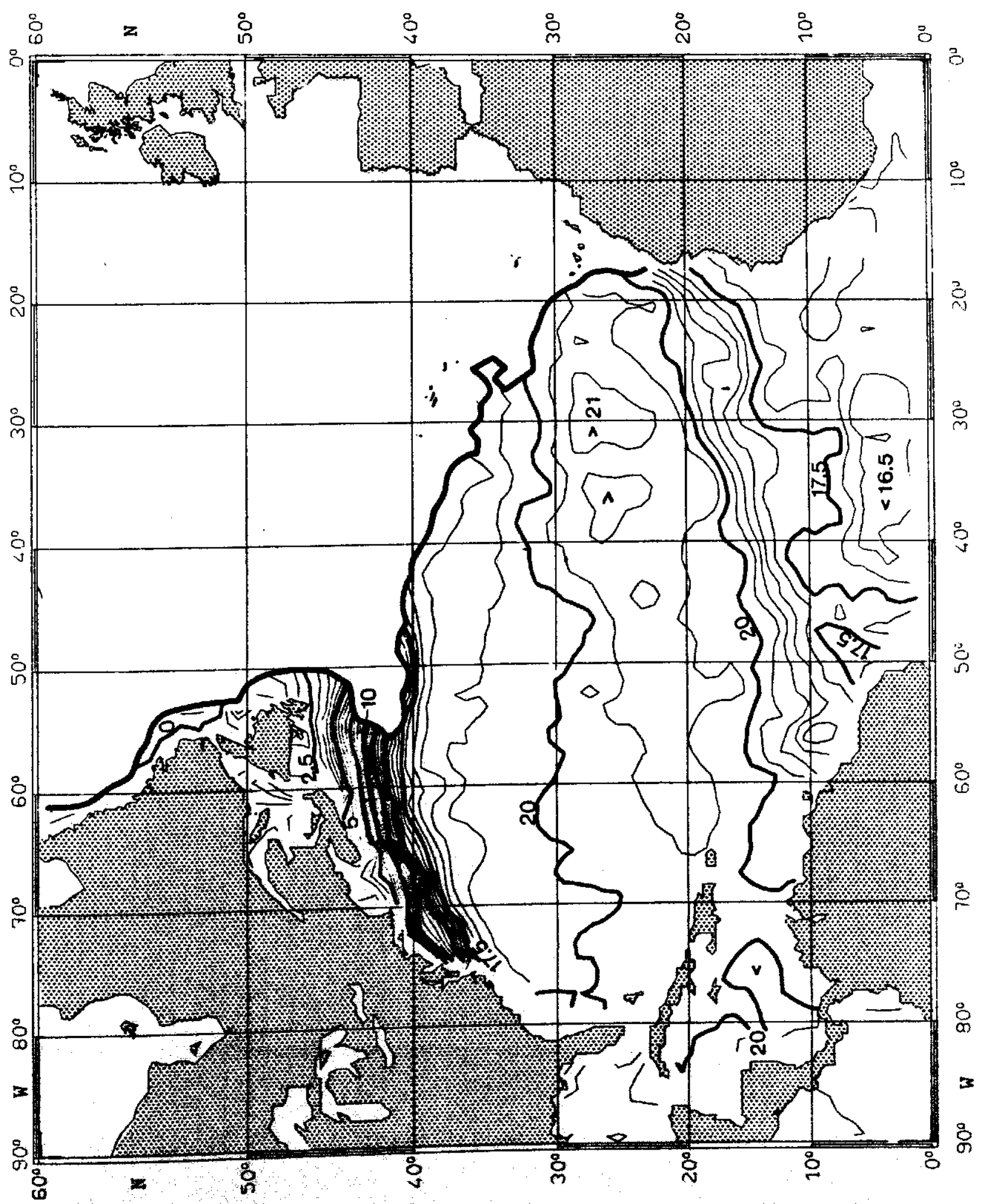


Fig. 132:

SALINITY $\left(10^{-3}\right)$ on $\sigma_{\theta}=26.0 \mathrm{~kg} \mathrm{~m}^{-3}$ DECEMBER

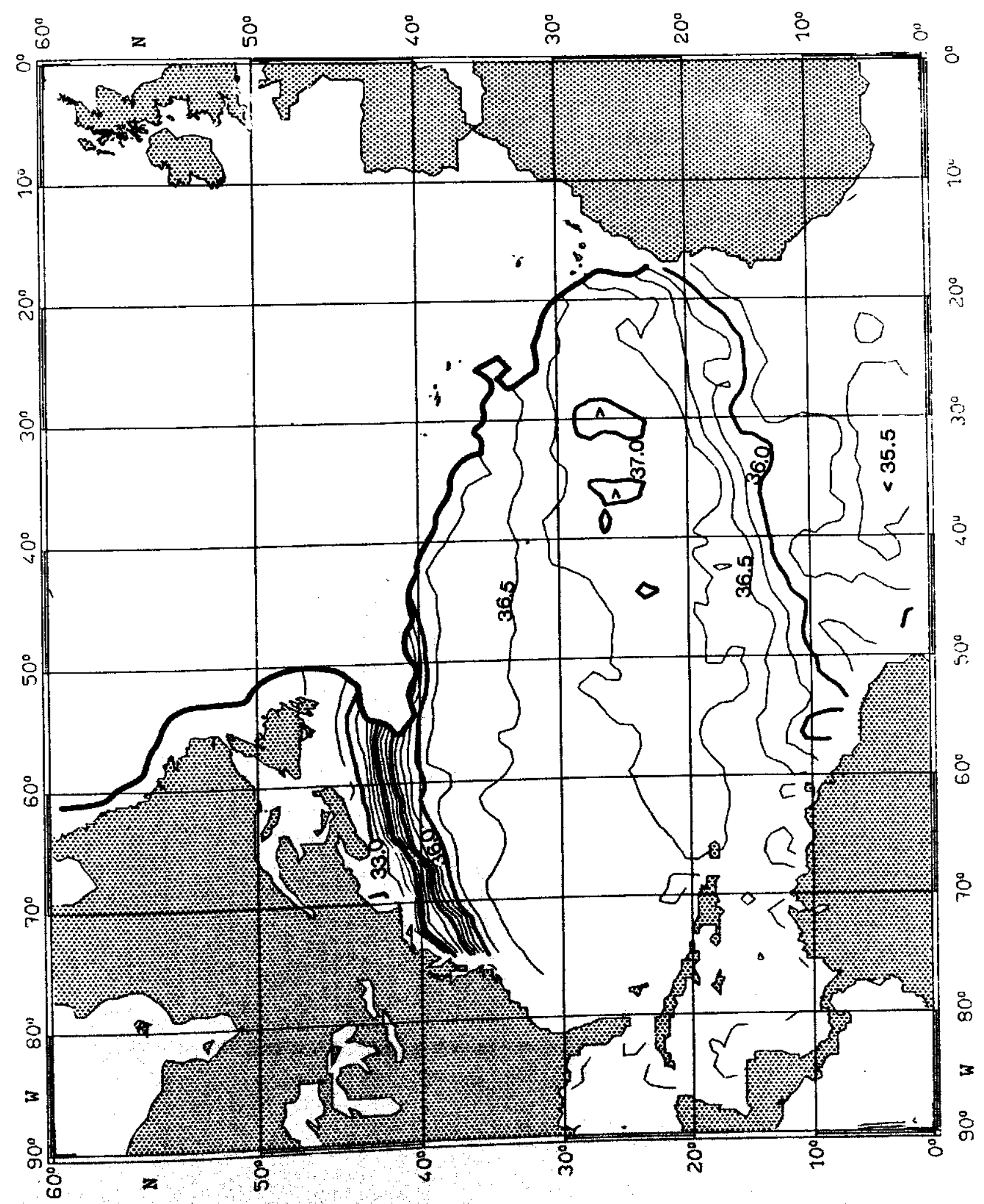


Fig. 133:

PRESSURE $\left(10^{4} \mathrm{~Pa}\right)$ on $\sigma_{\theta}=27.0 \mathrm{~kg} \mathrm{~m}^{-3}$ DECEMBER

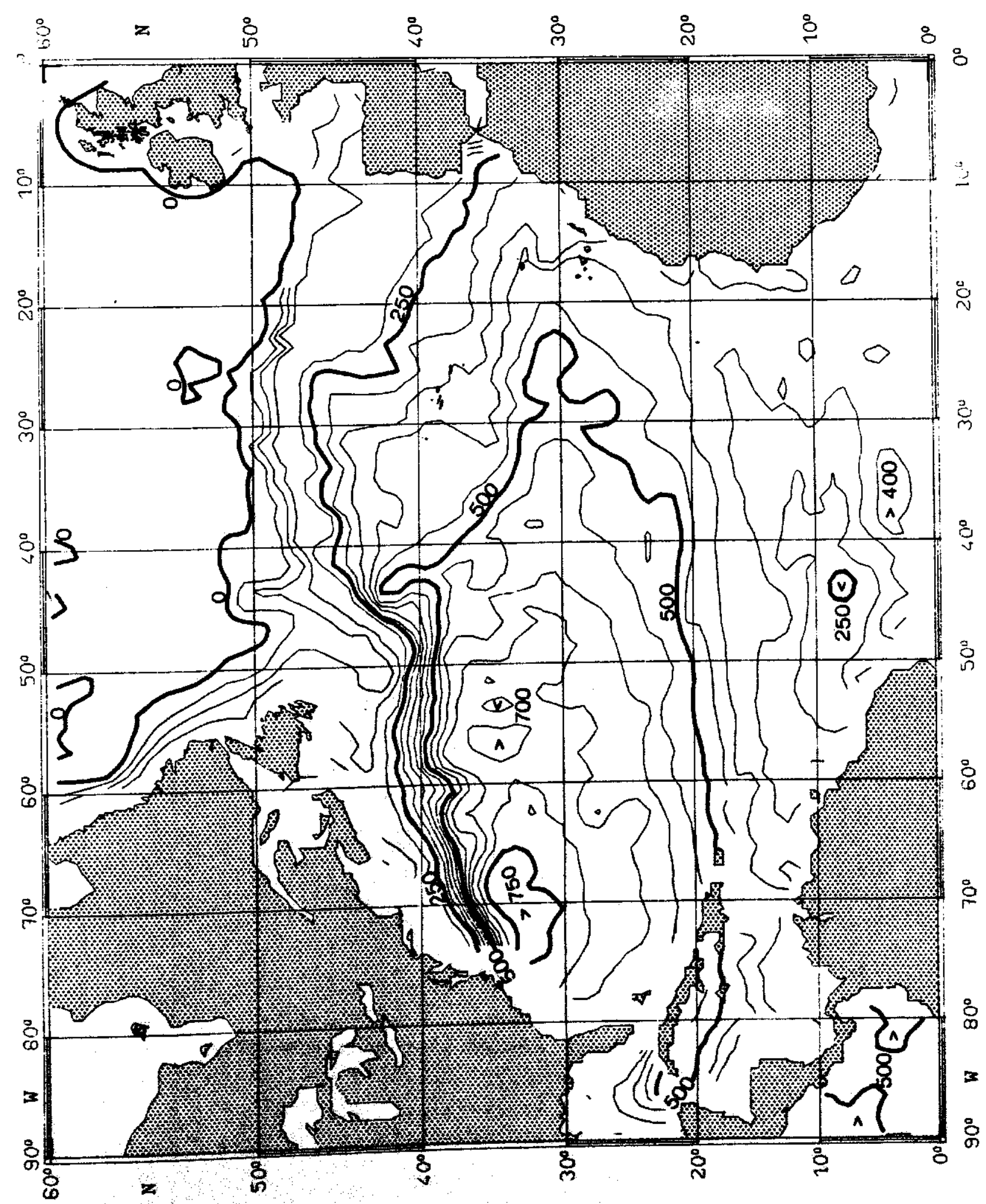


Fig. 134:

TEMPERATURE $\left({ }^{\circ} \mathrm{C}\right)$ on $\sigma_{\theta}=27.0 \mathrm{~kg} \mathrm{~m}^{-3}$ DECEMBER

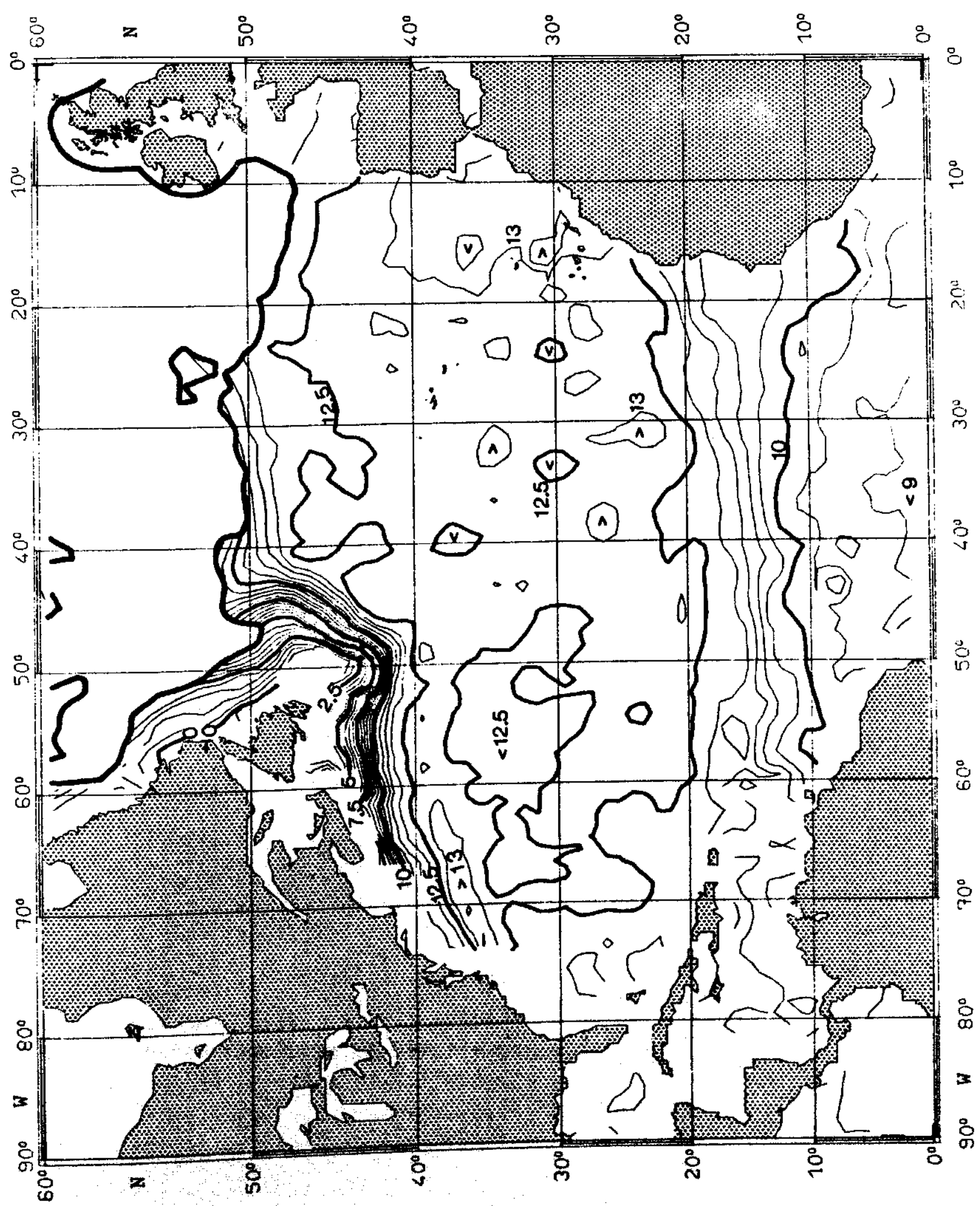


Fig. 135:

SALINITY $\left(10^{-3}\right)$ on $\sigma_{\theta}=27.0 \mathrm{~kg} \mathrm{~m}^{-3} \quad$ DECEMBER

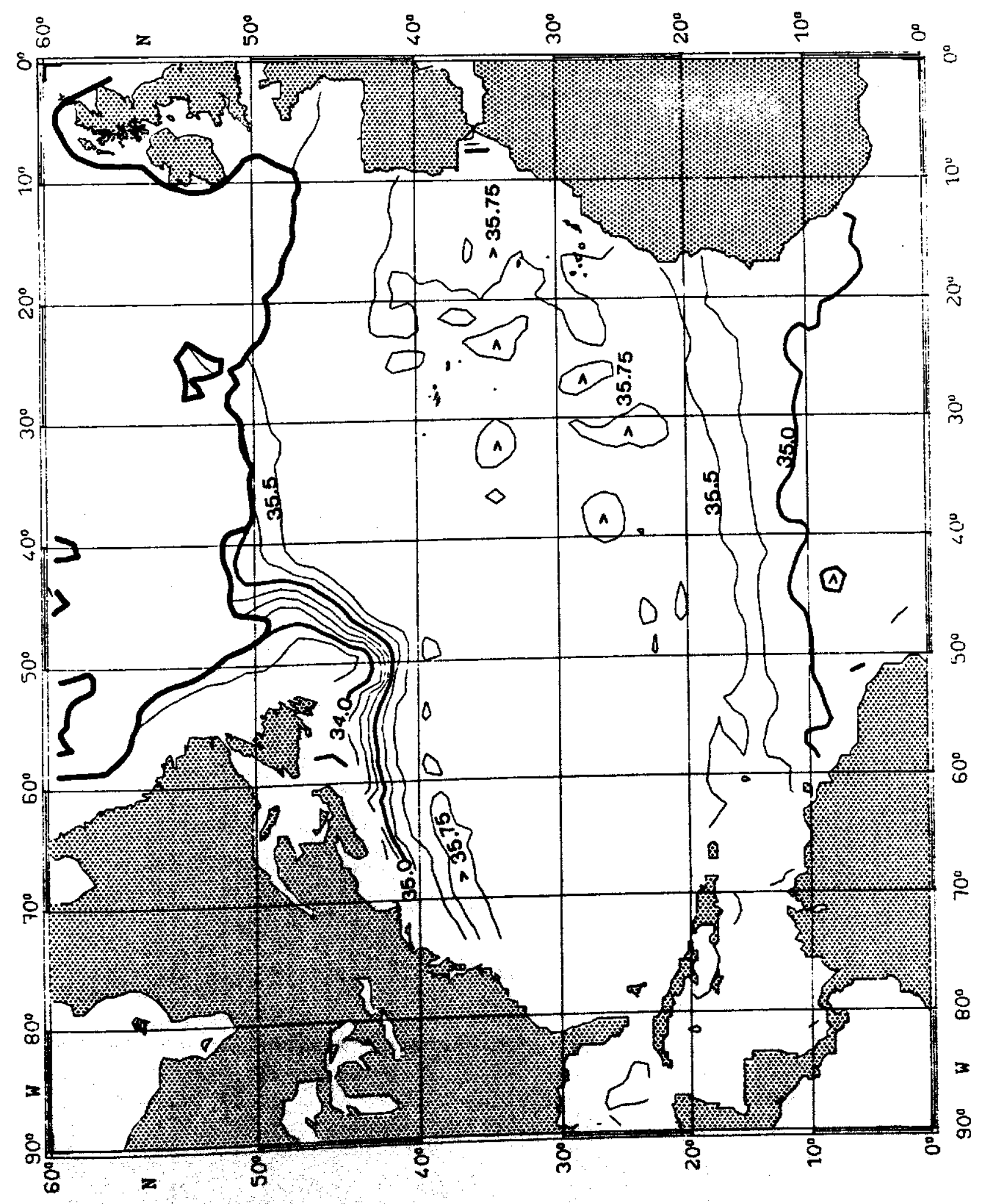


Fig. 136: Location of vertical sections

Azores - Greenland and Azores - English Channel

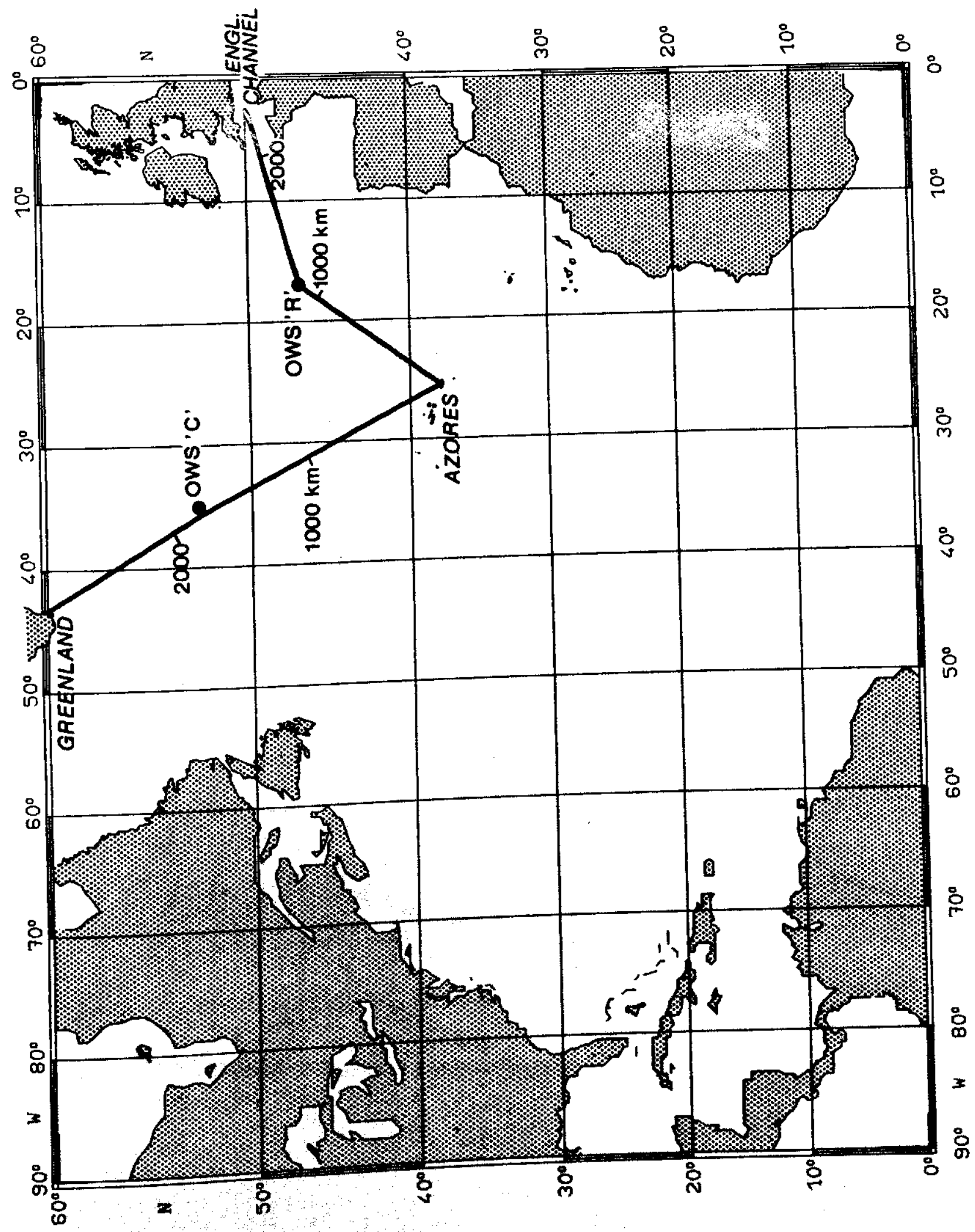




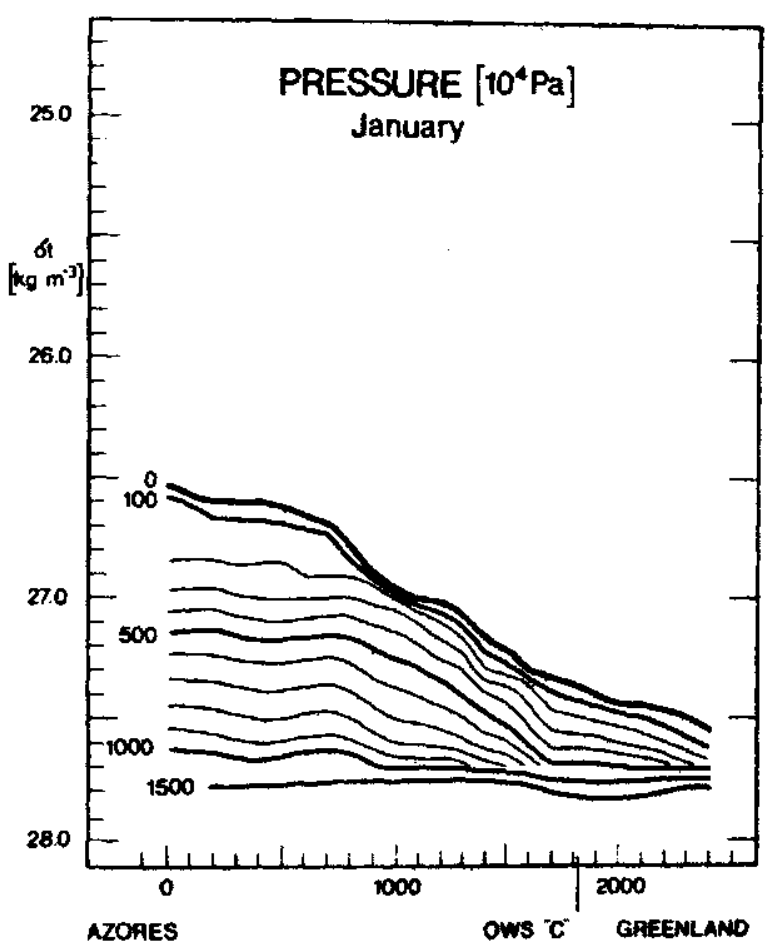

Fig. 137:

$$
\begin{gathered}
\text { AZORES - GREENLAND } \\
\text { January }
\end{gathered}
$$
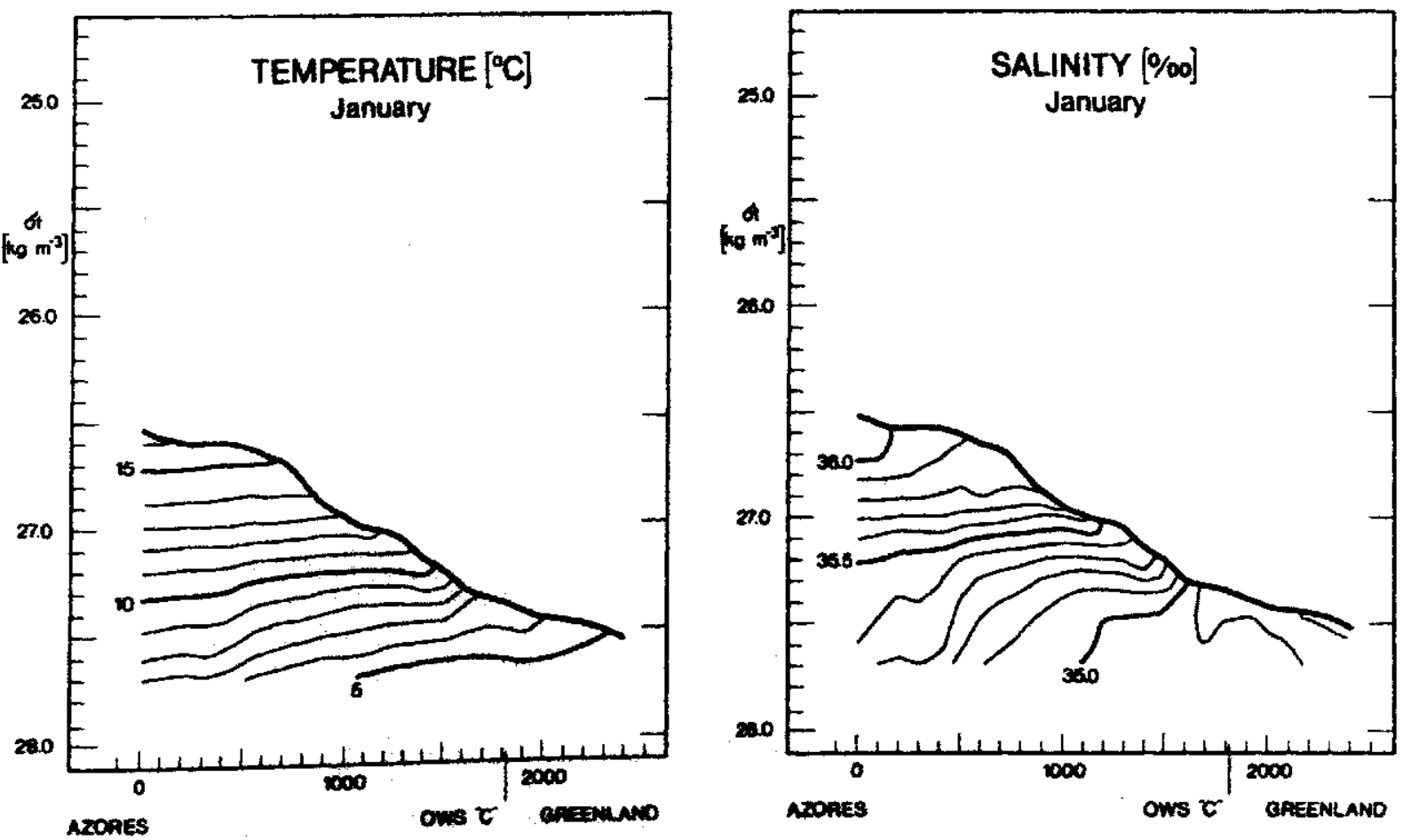


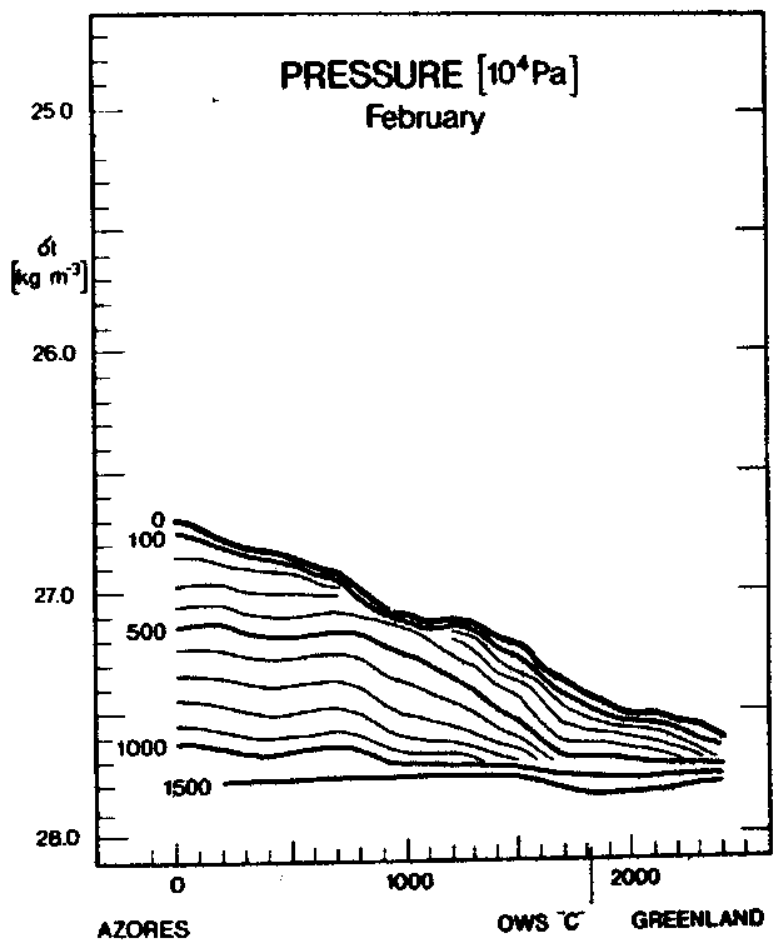

Fig. 138:
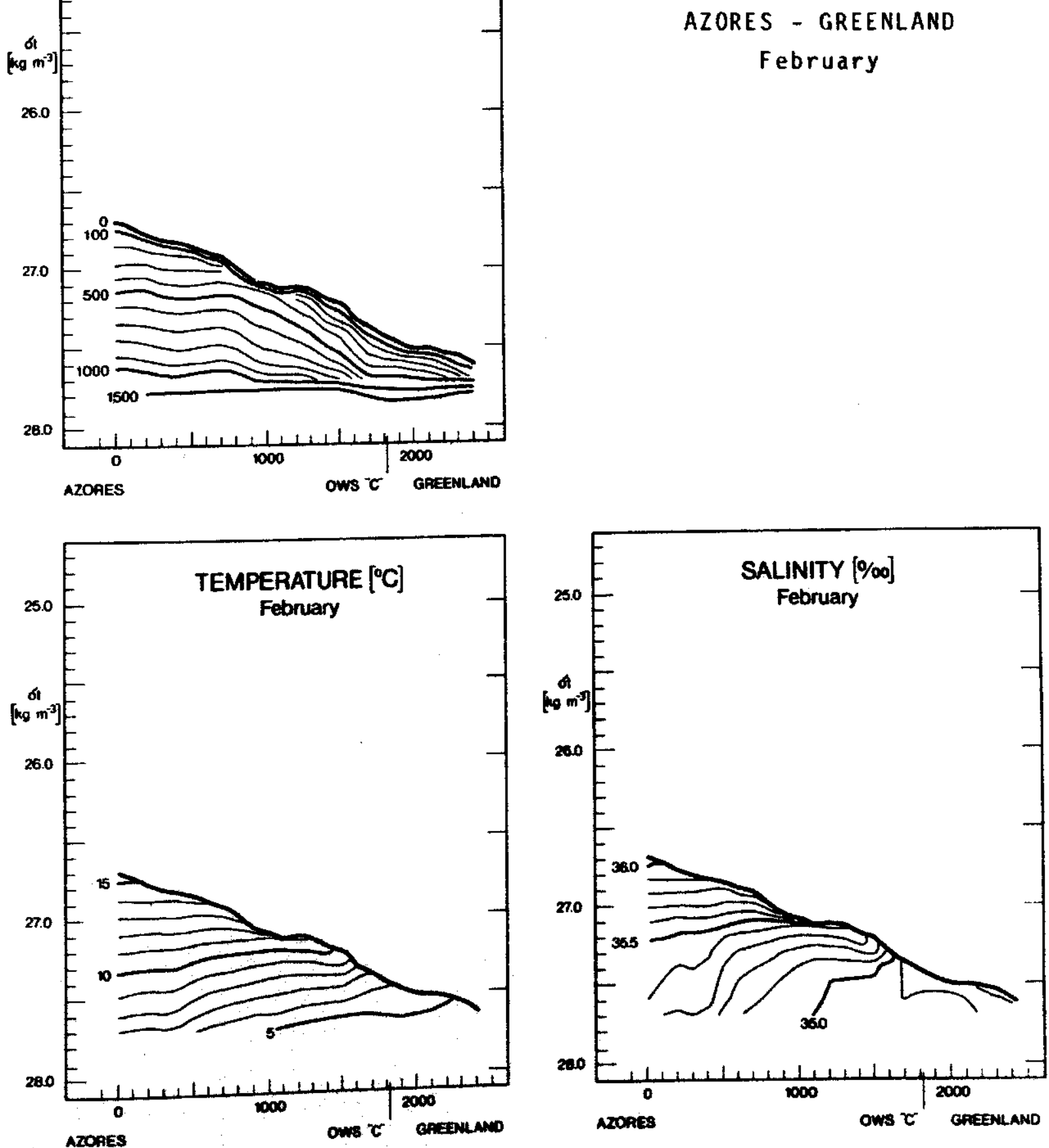


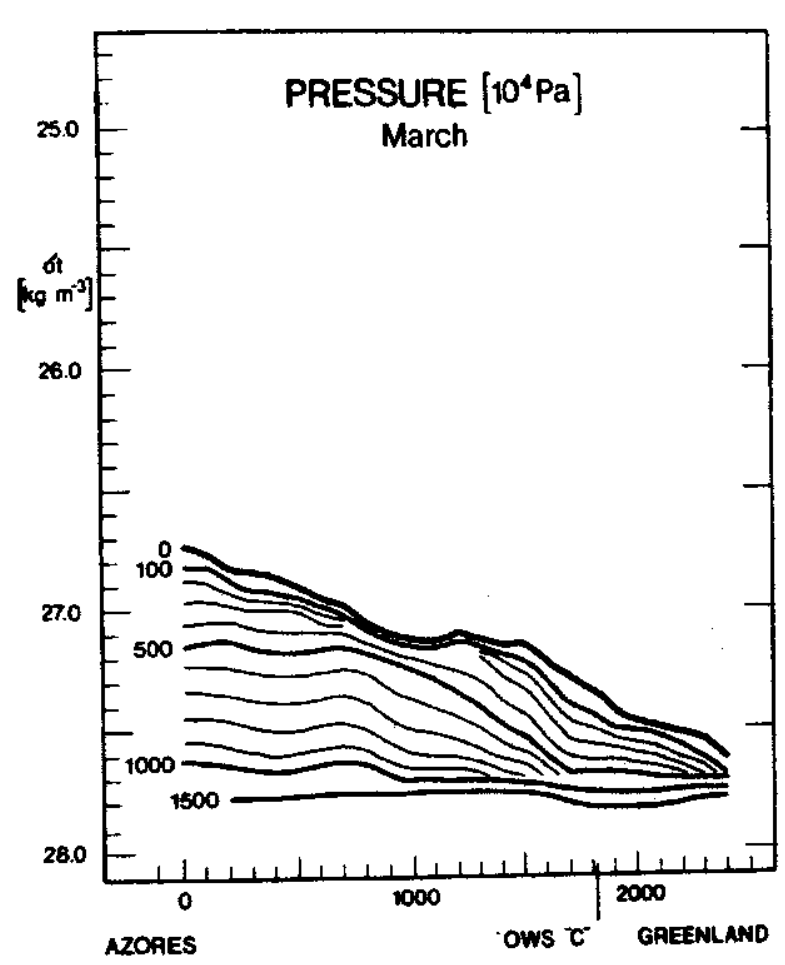

Fig. 139:

AZORES - GREENLAND

March
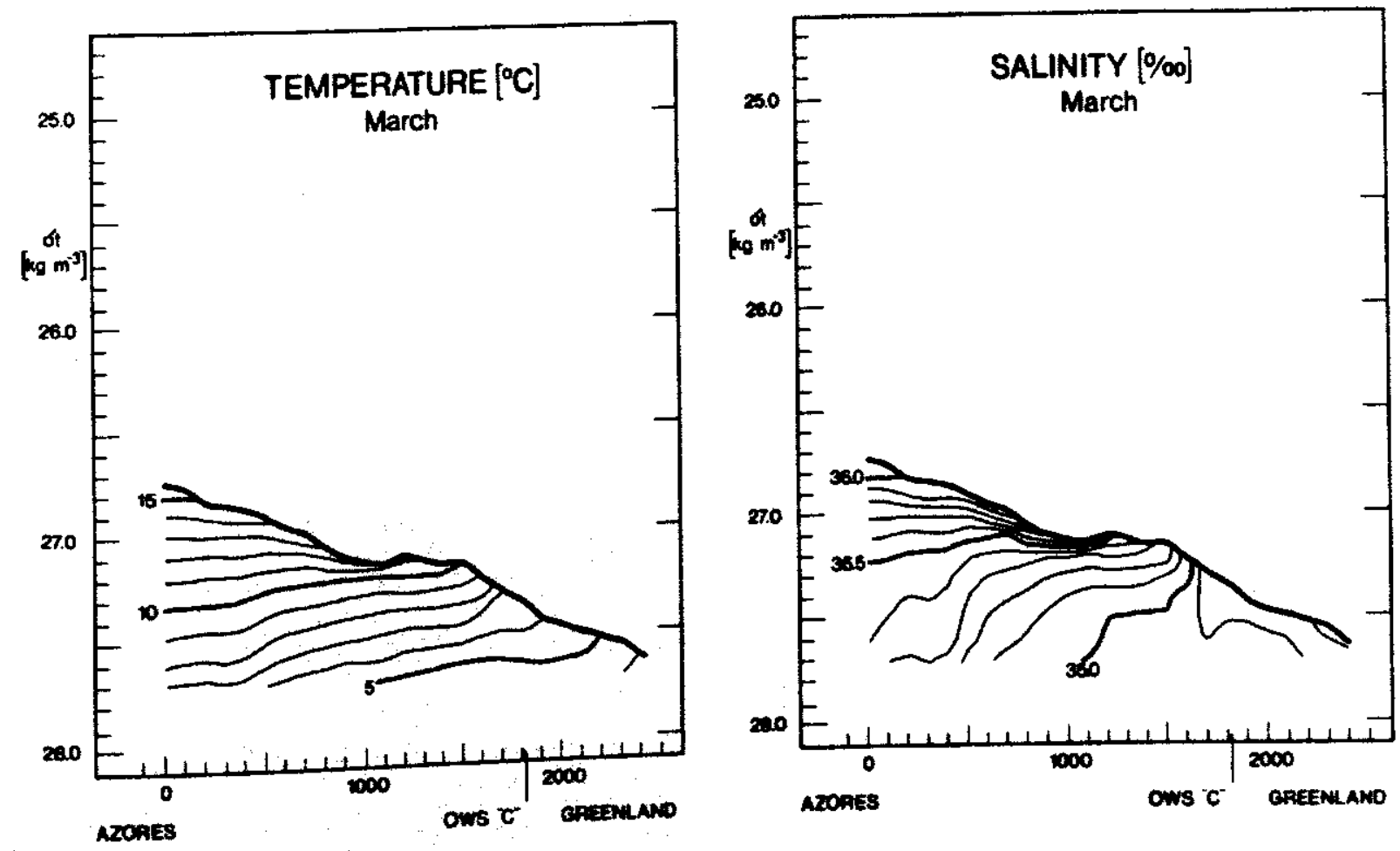


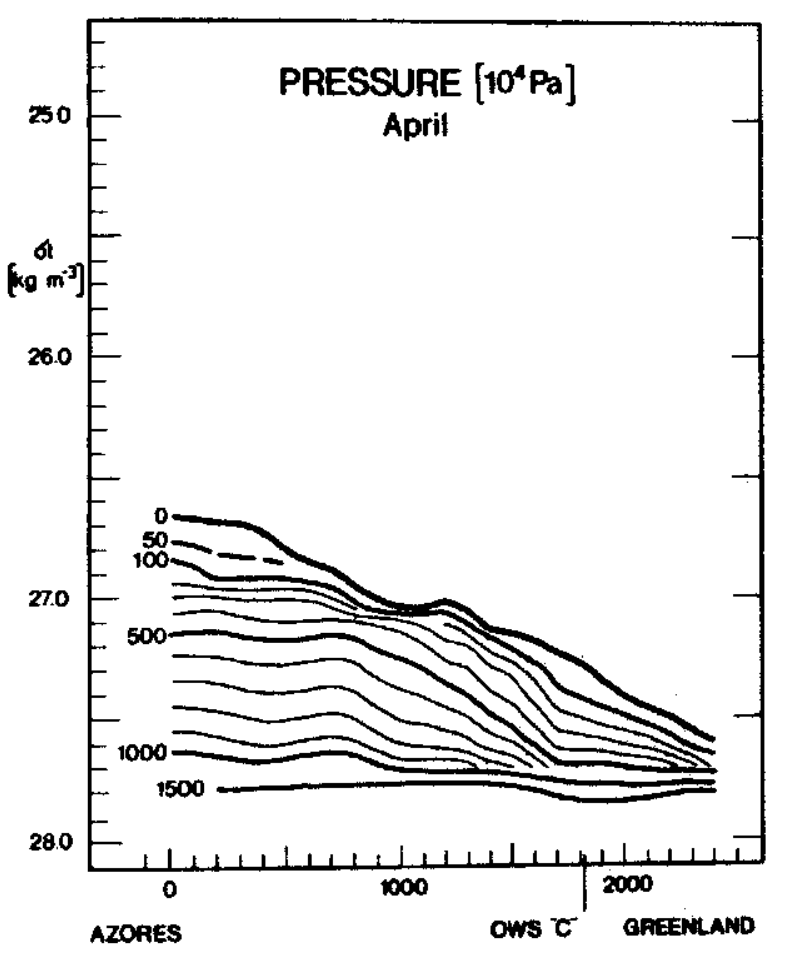

Fig. 140:

\section{AZORES - GREENLAND \\ April}
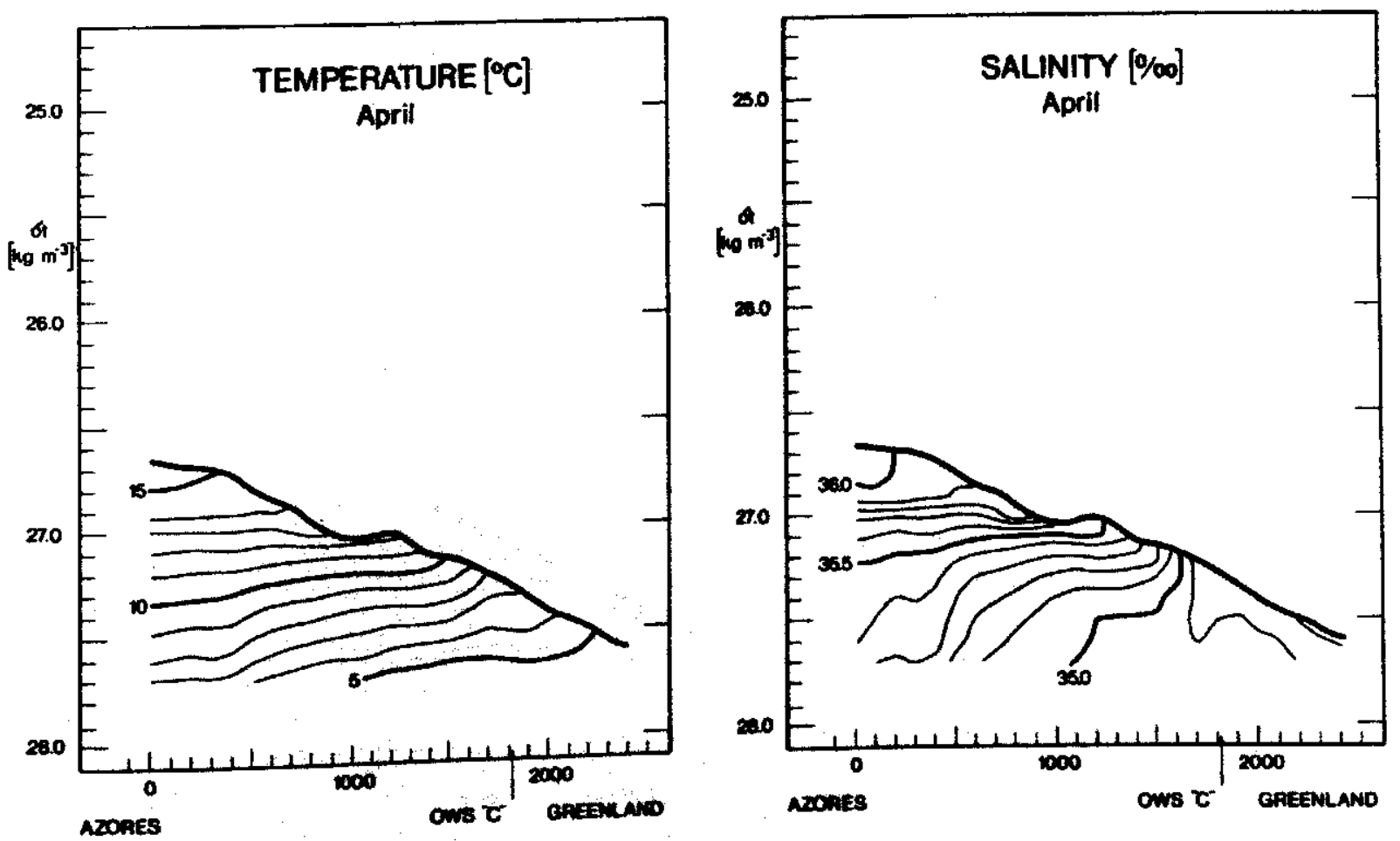


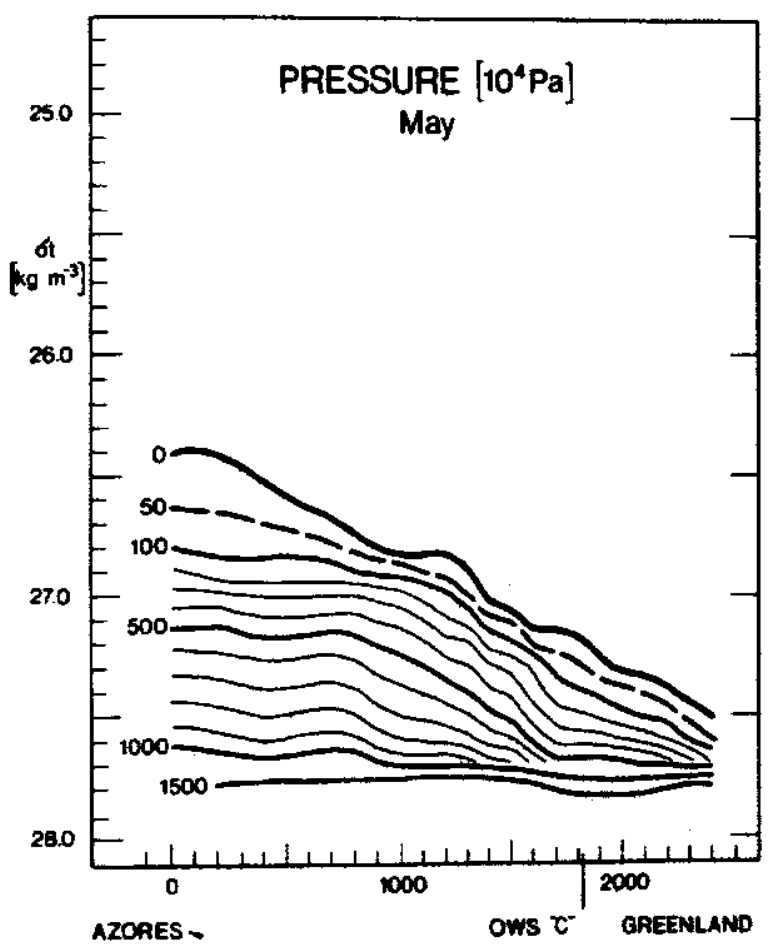

Fig. 141:

\section{AZORES - GREENLAND \\ May}
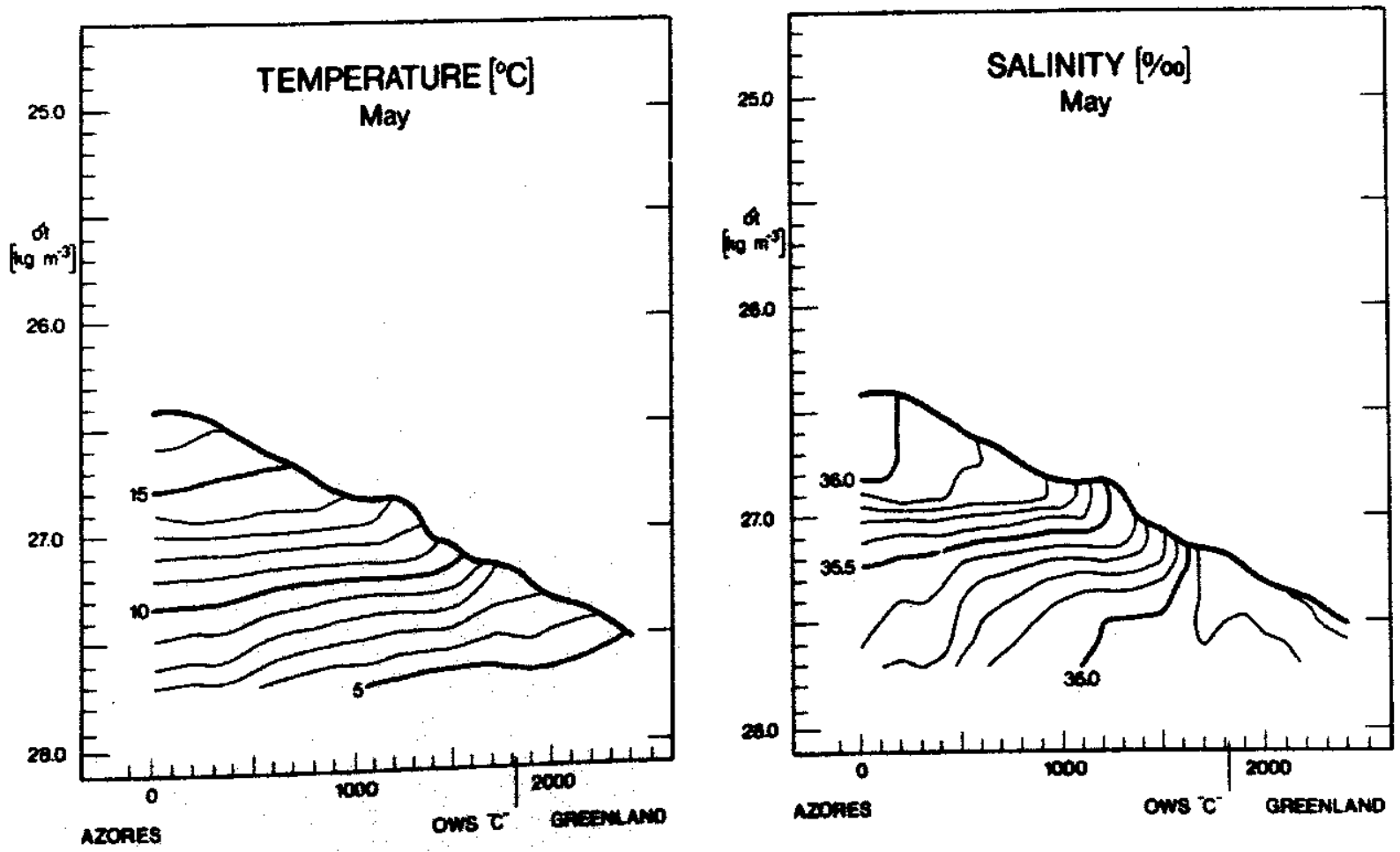


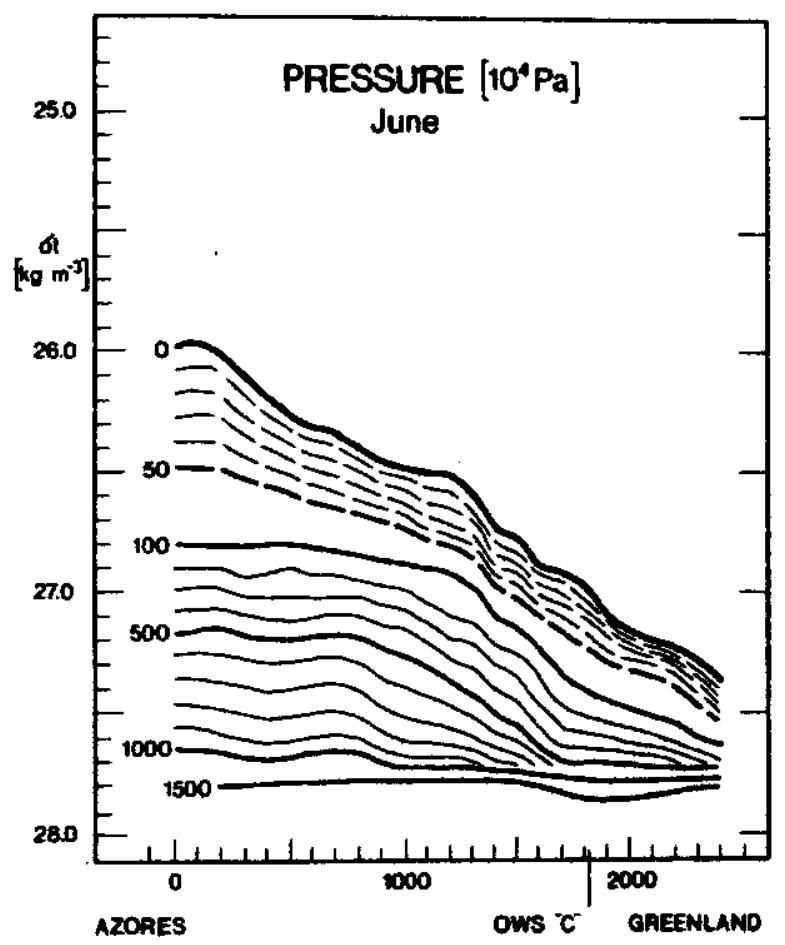

Fig. 142:
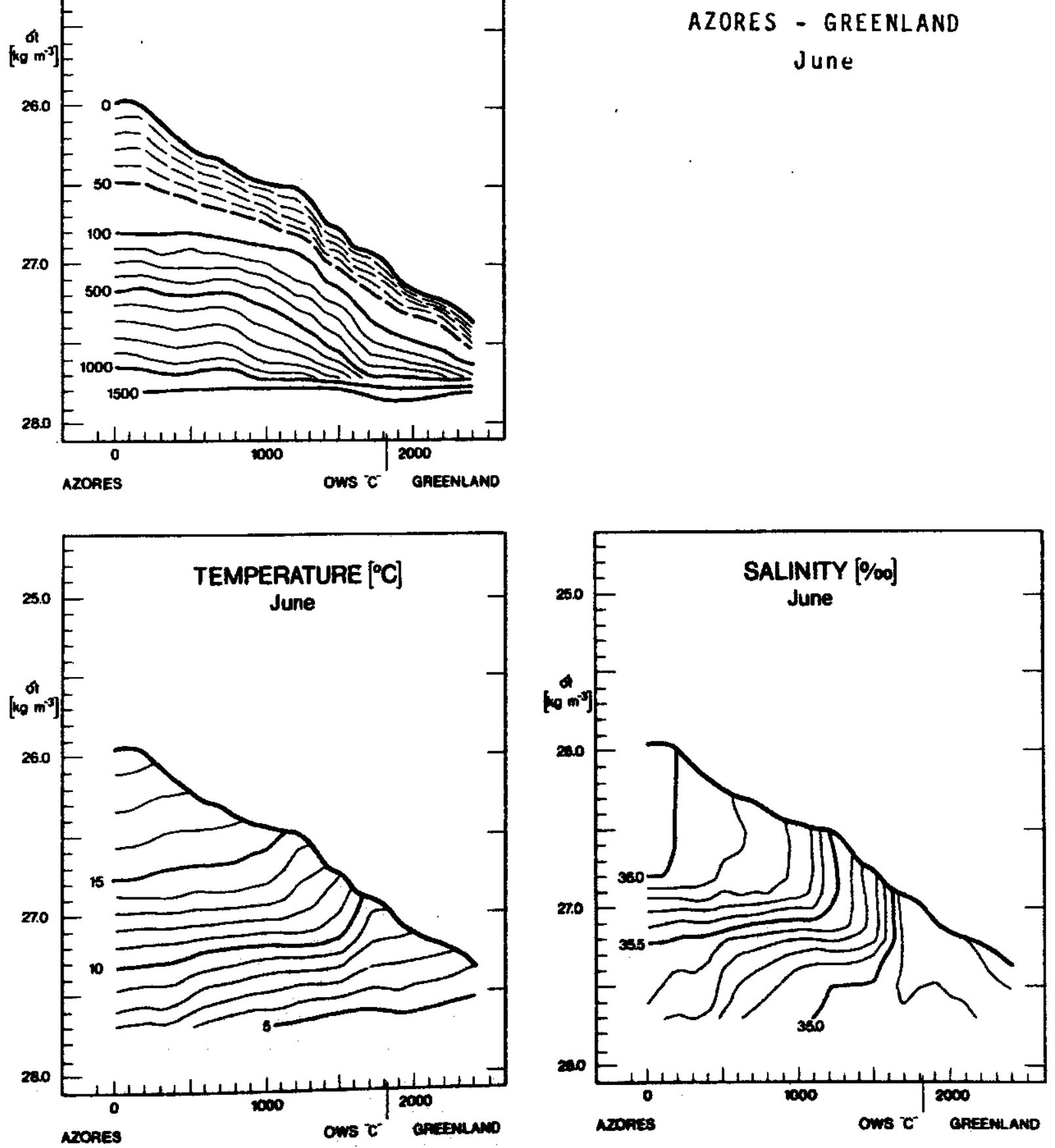


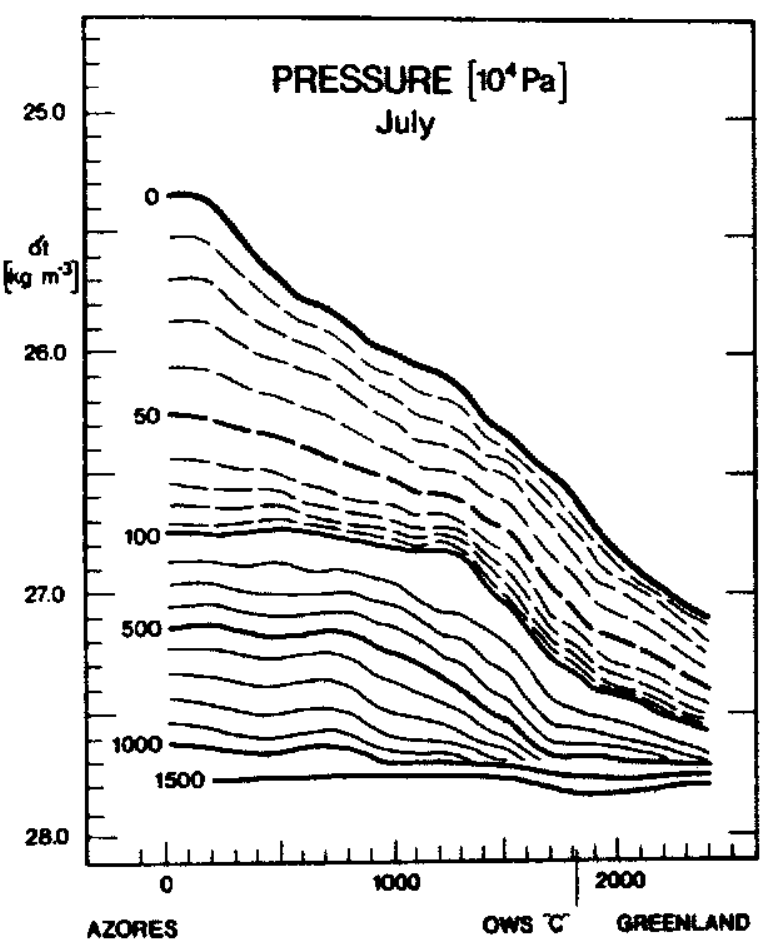

Fig. 143:

$$
\begin{gathered}
\text { AZORES - GREENLAND } \\
\text { July }
\end{gathered}
$$
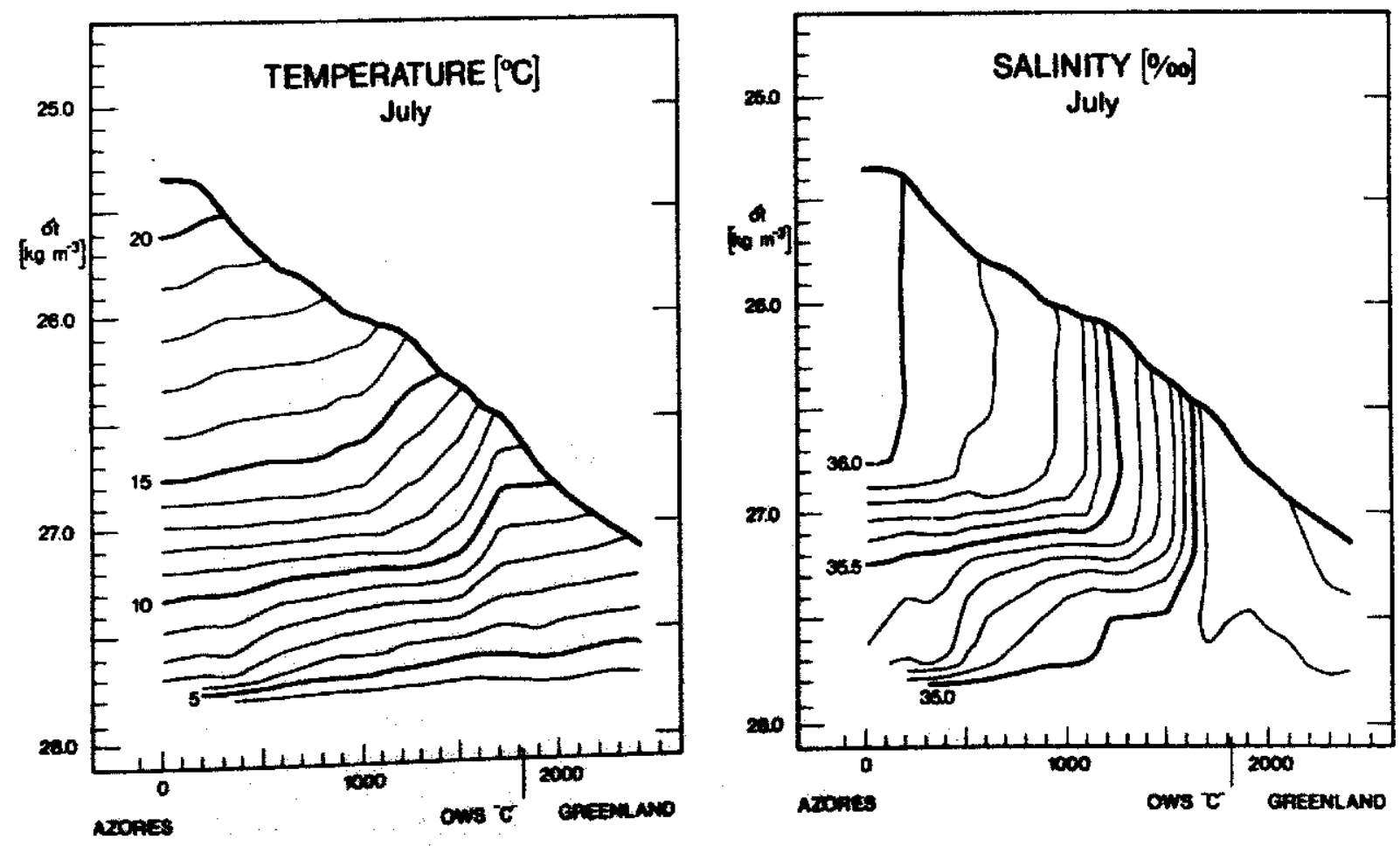


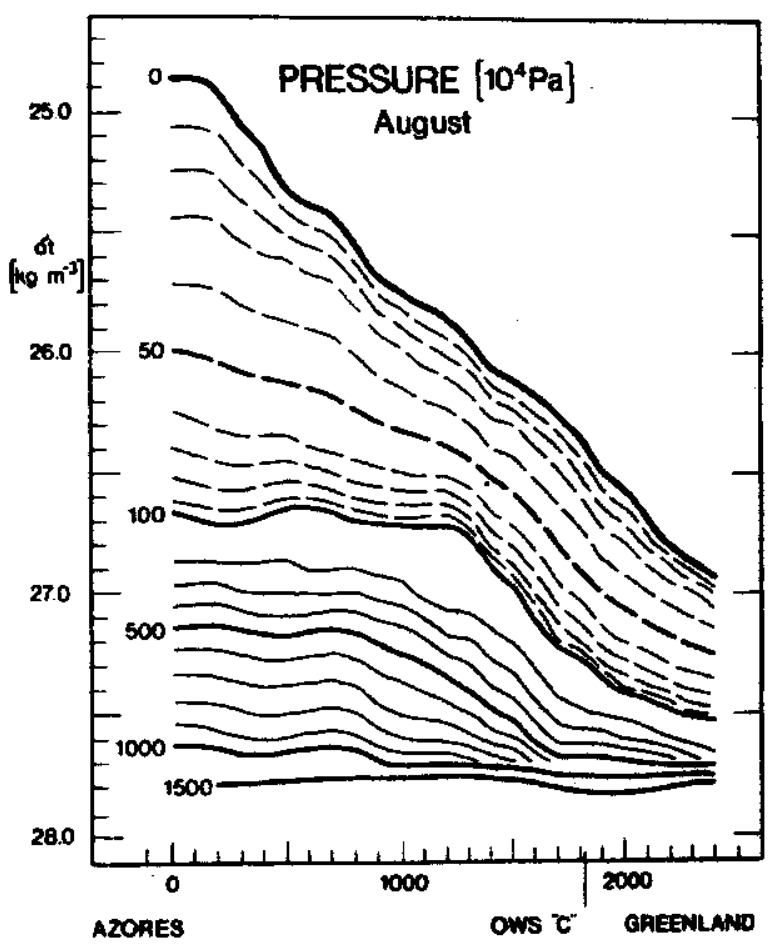

Fig. 144:

\section{AZORES - GREENLAIID \\ August}
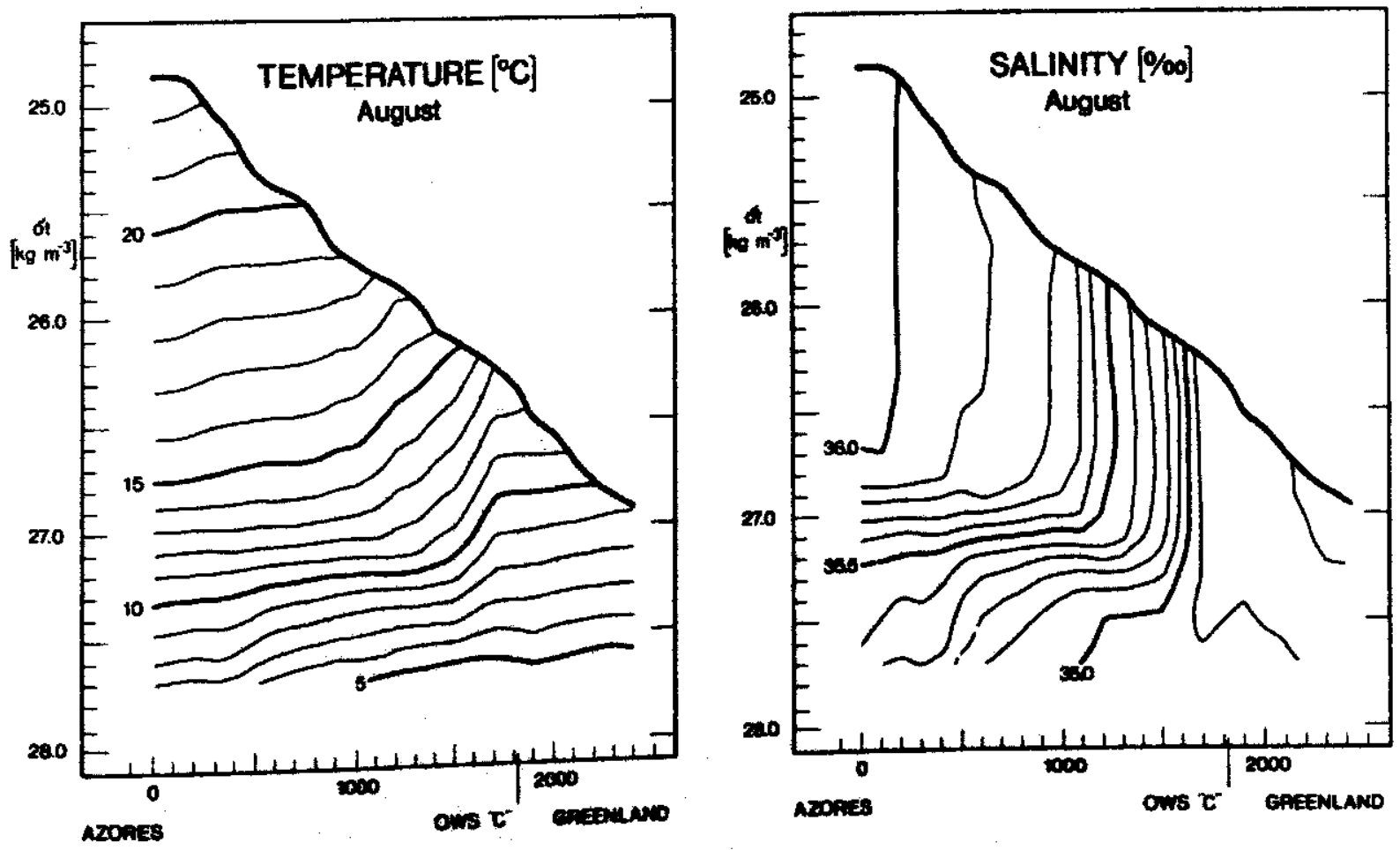


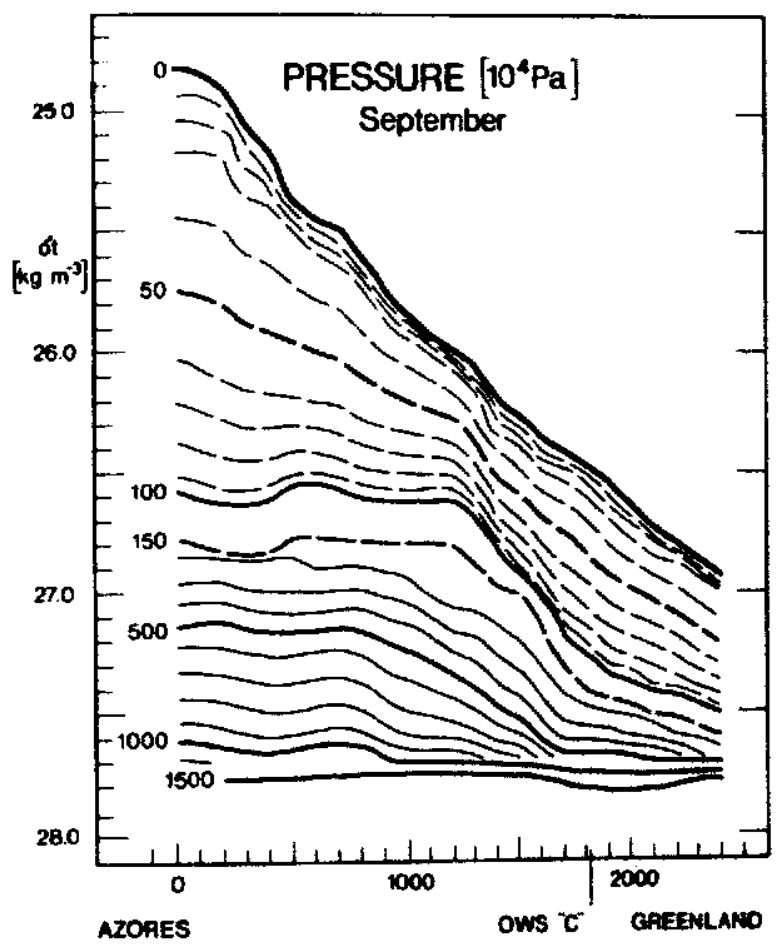

Fig. 145:

$$
\begin{gathered}
\text { AZORES - GREENLAND } \\
\text { September }
\end{gathered}
$$
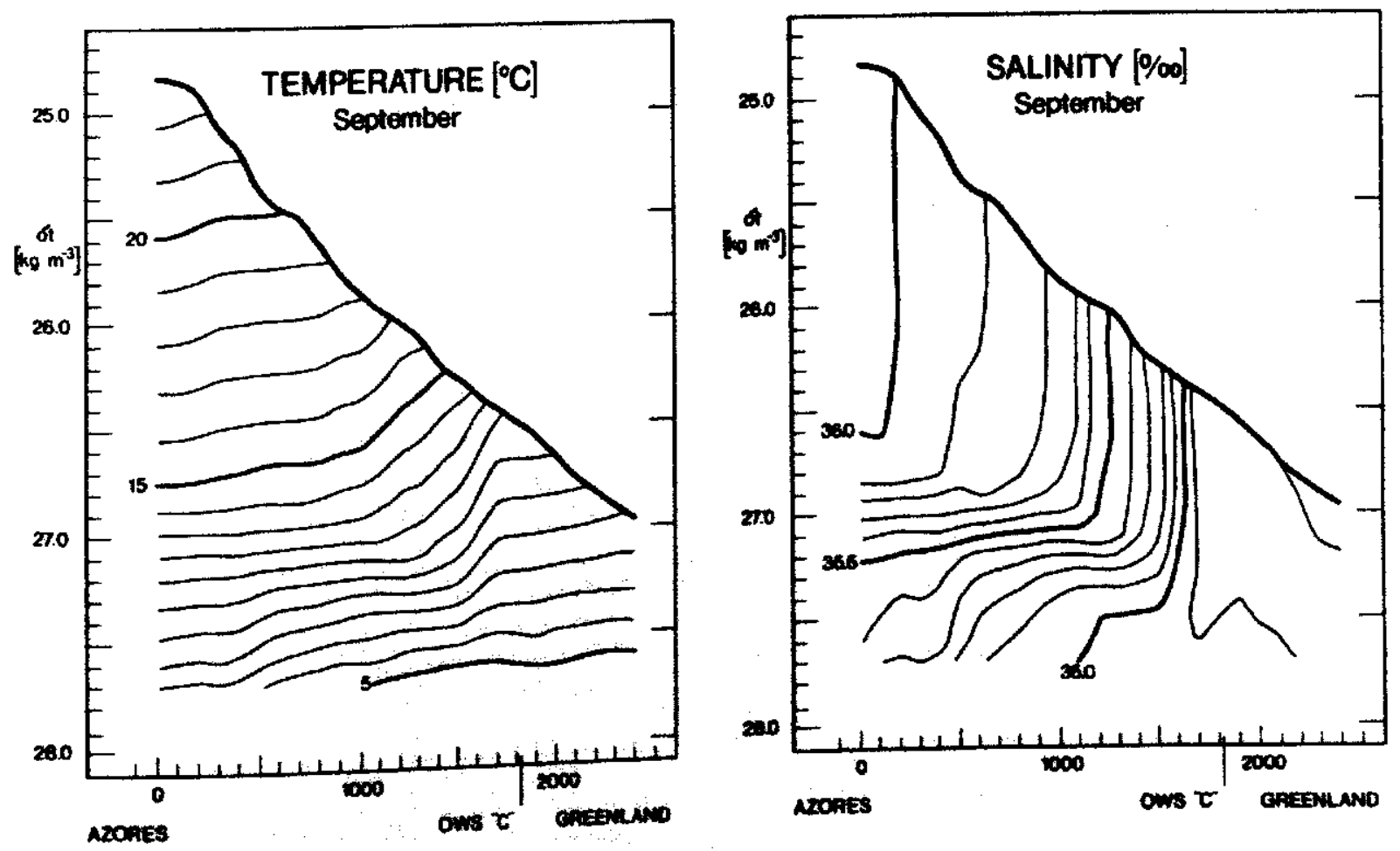


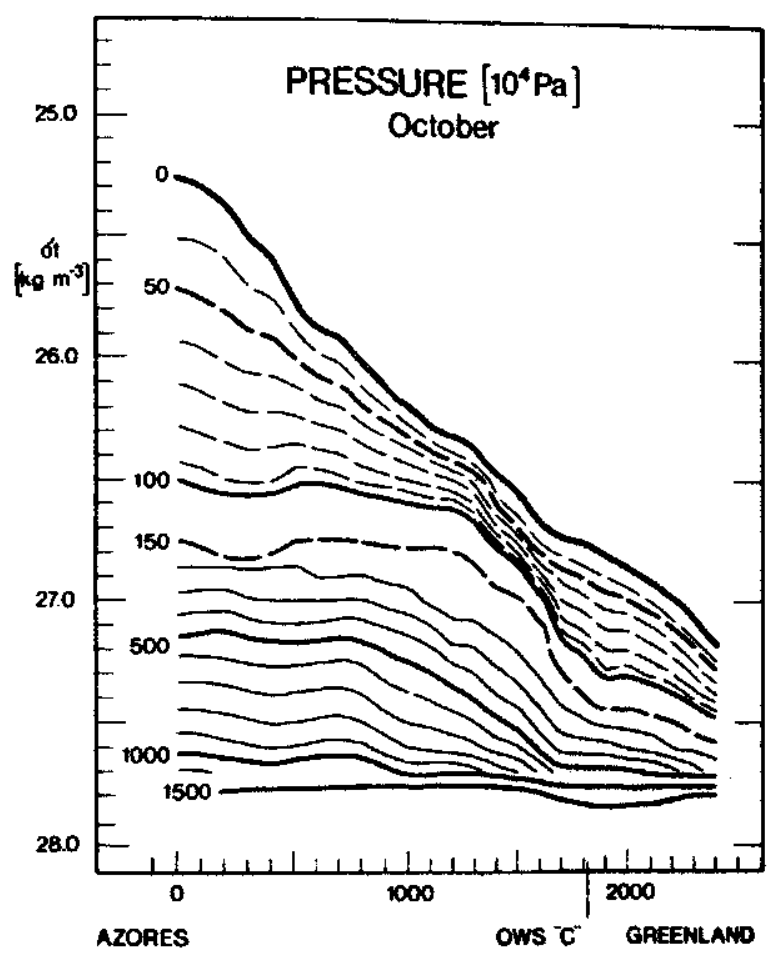

Fig. 146:

\section{AZORES - GREENLAND \\ October}
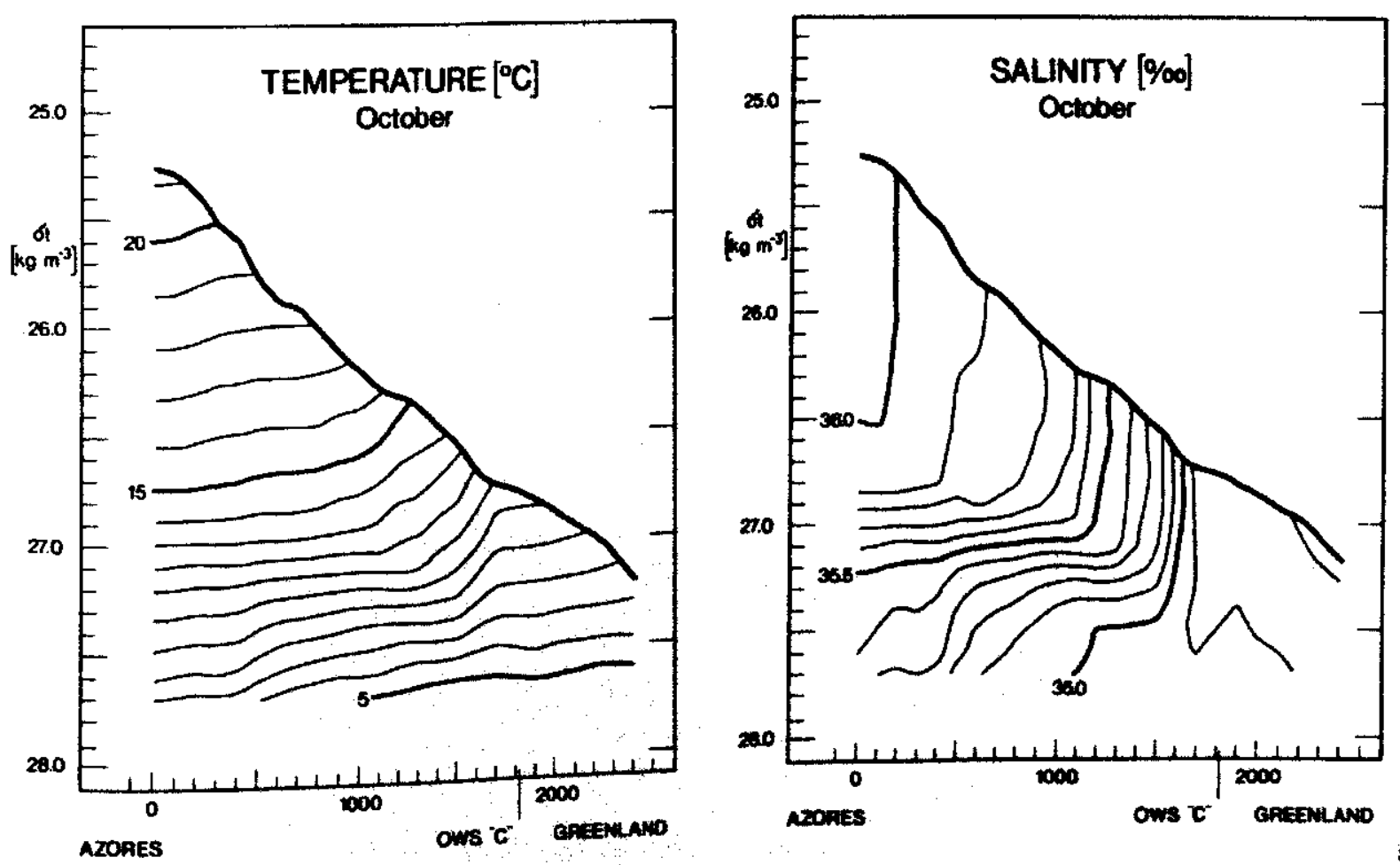


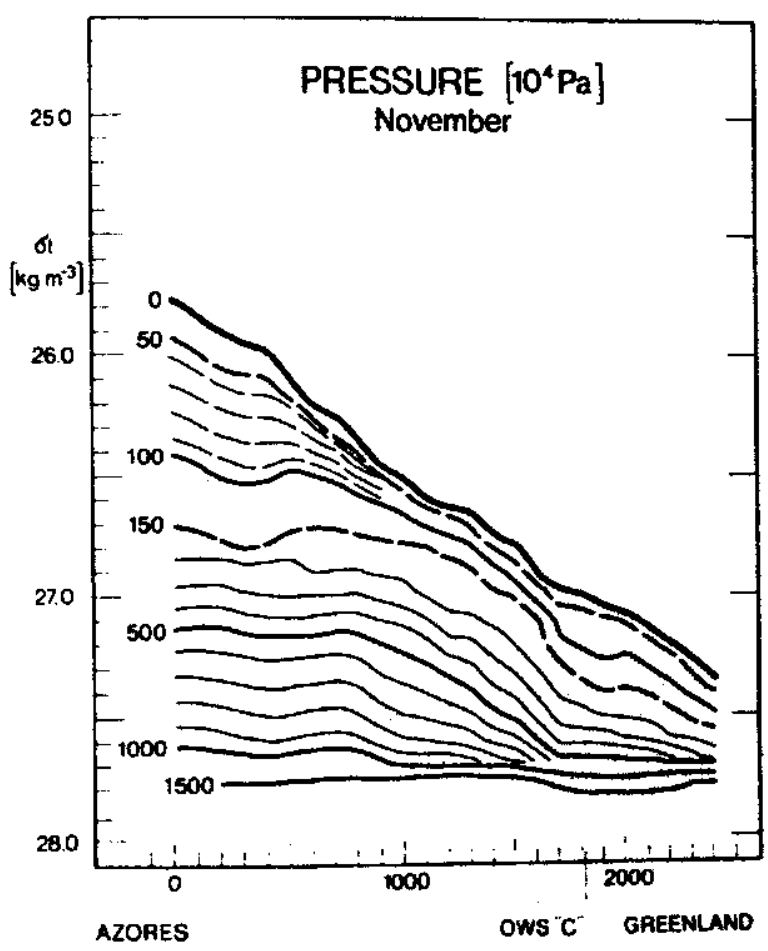

Fig. 147:

\section{AZORES - GREENLAND}

November
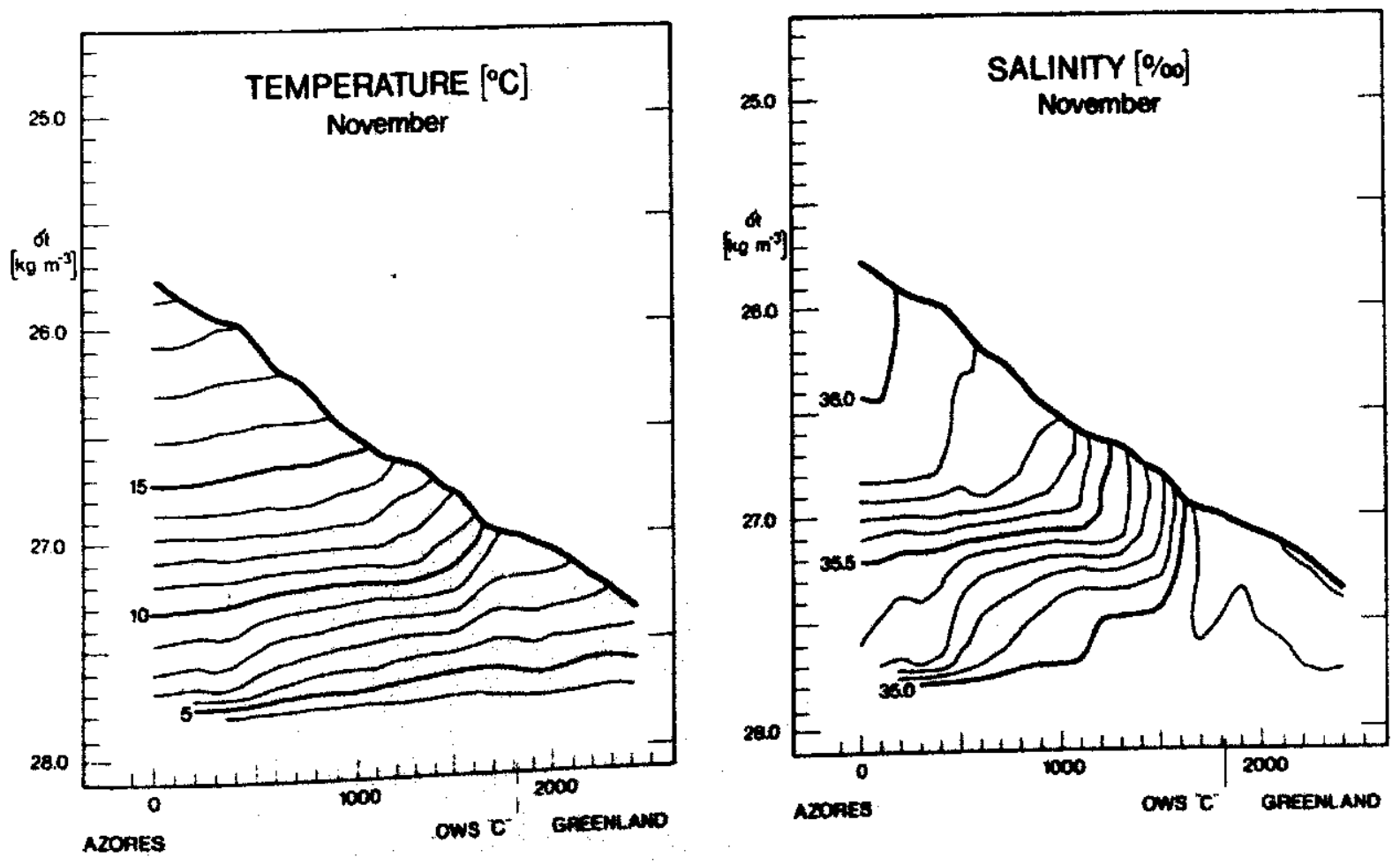


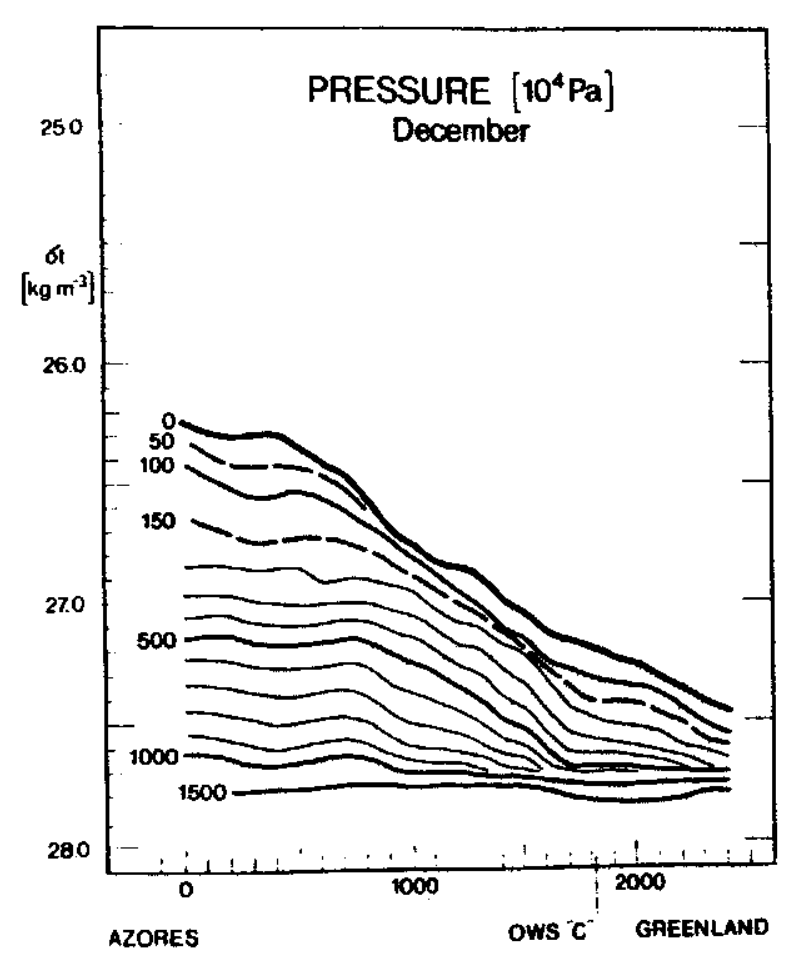

Fig. 148:

\section{AZORES - GREENLAND \\ December}
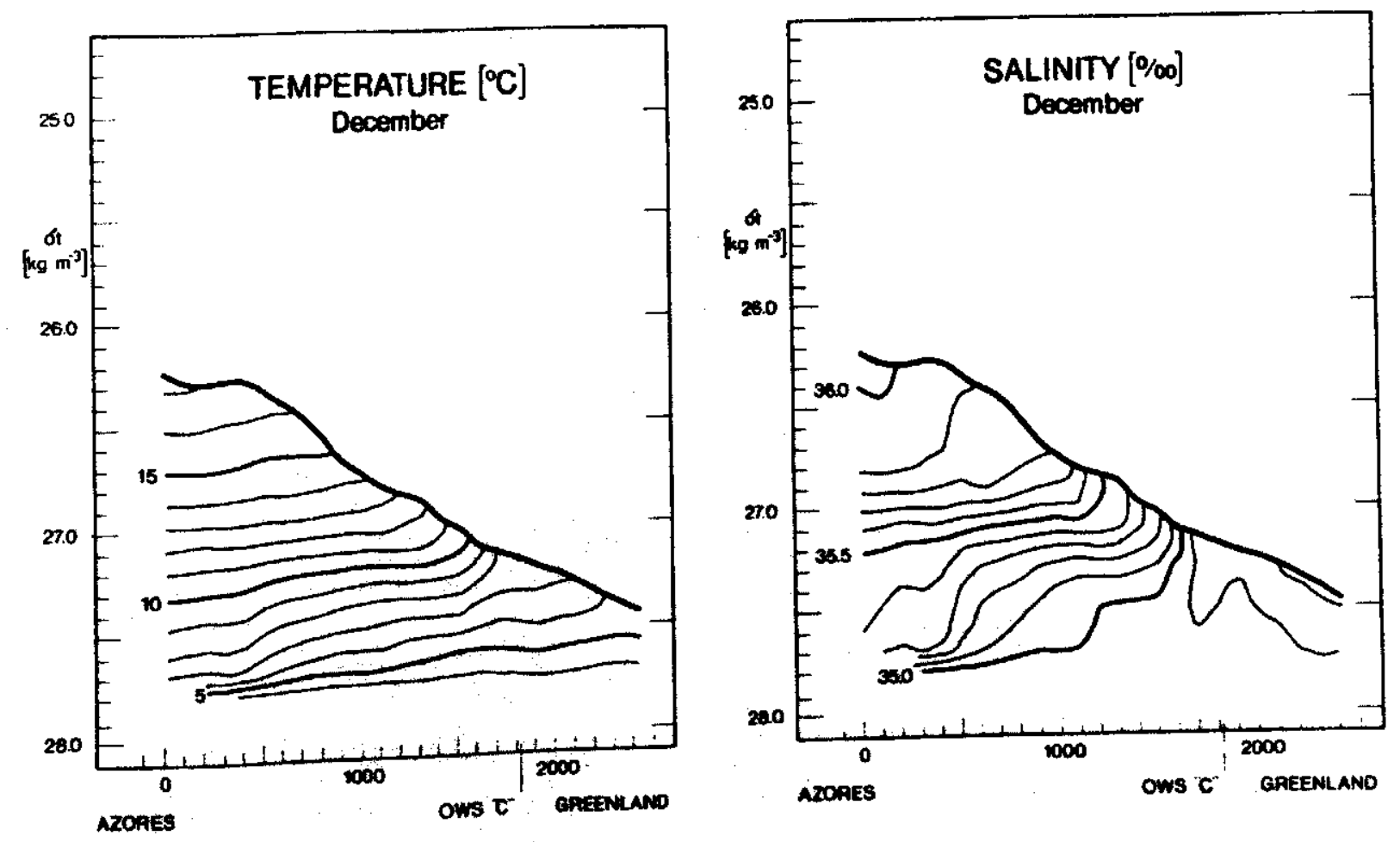


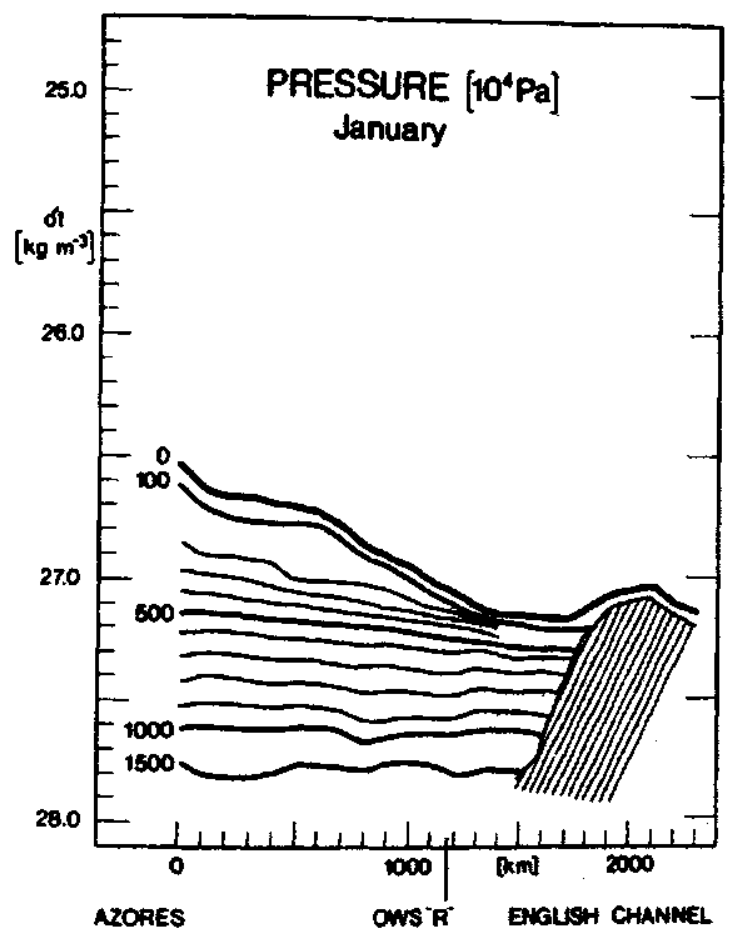

Fig. 149:

\section{AZORES - ENGLISH CHANNEL January}
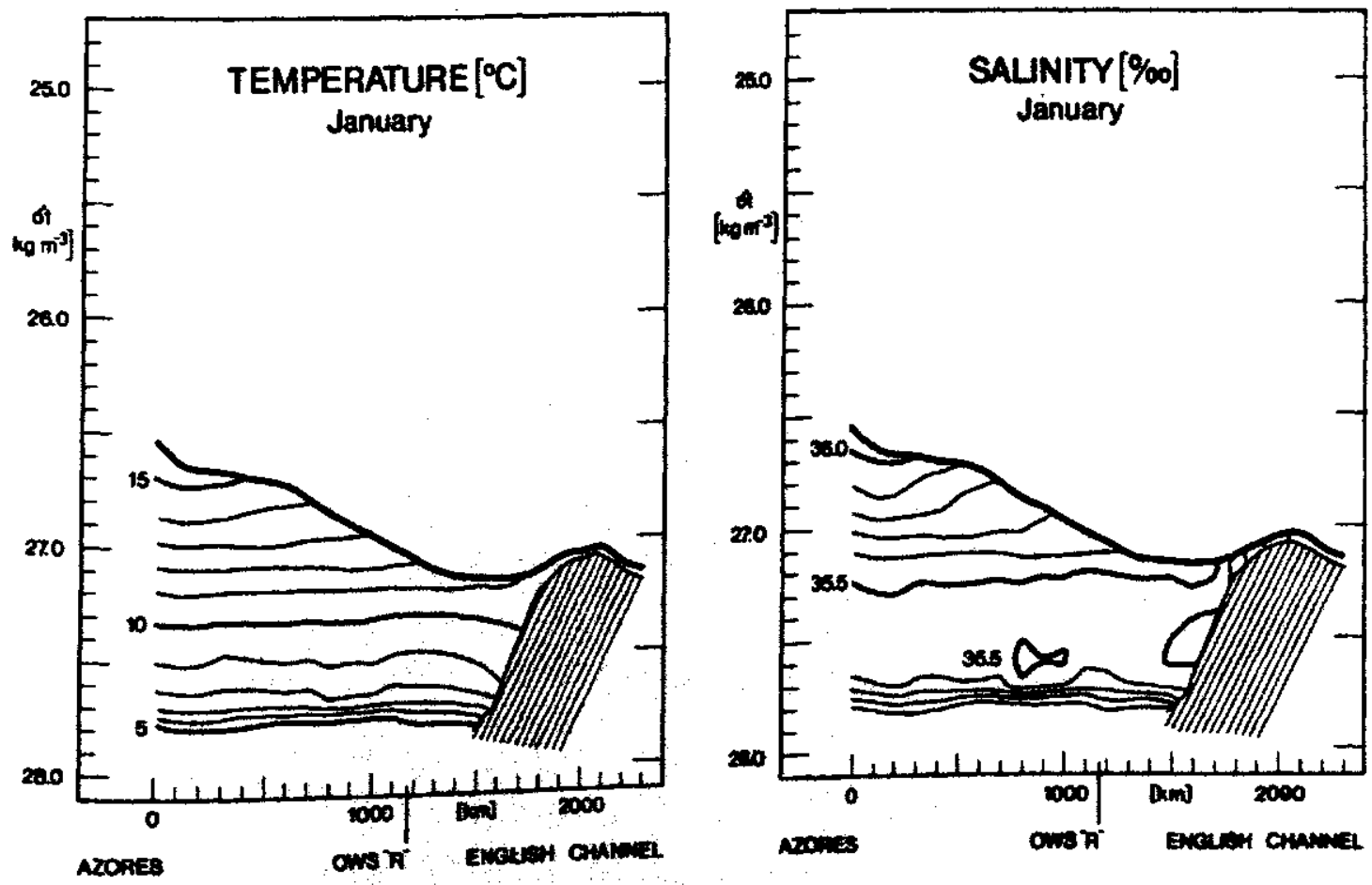


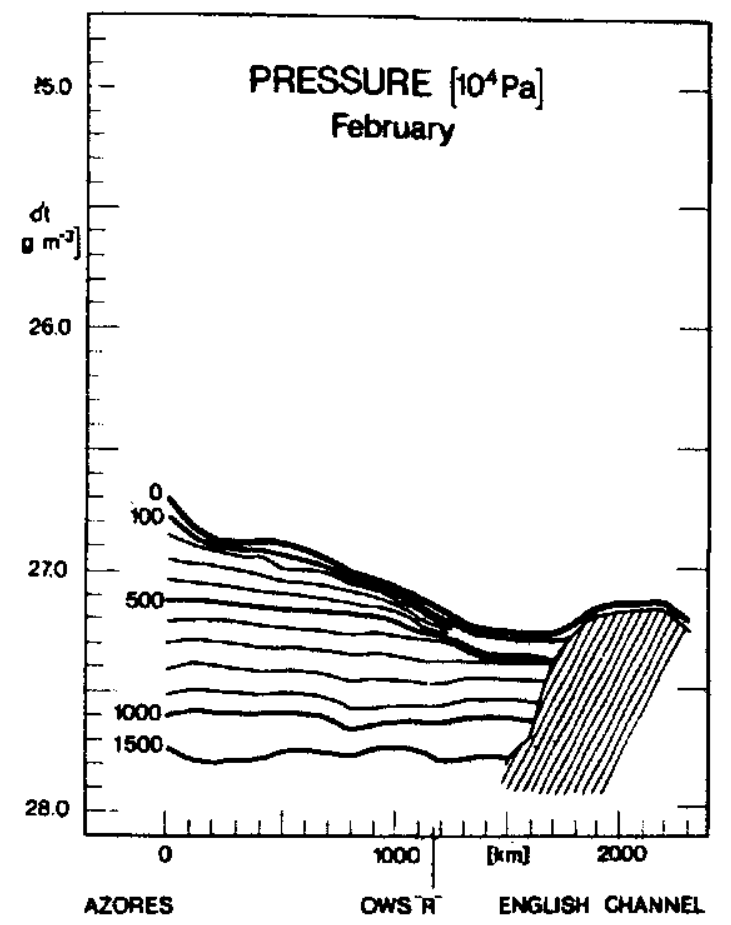

Fig. 150:

\section{AZORES - ENGLISH CHANNEL \\ February}
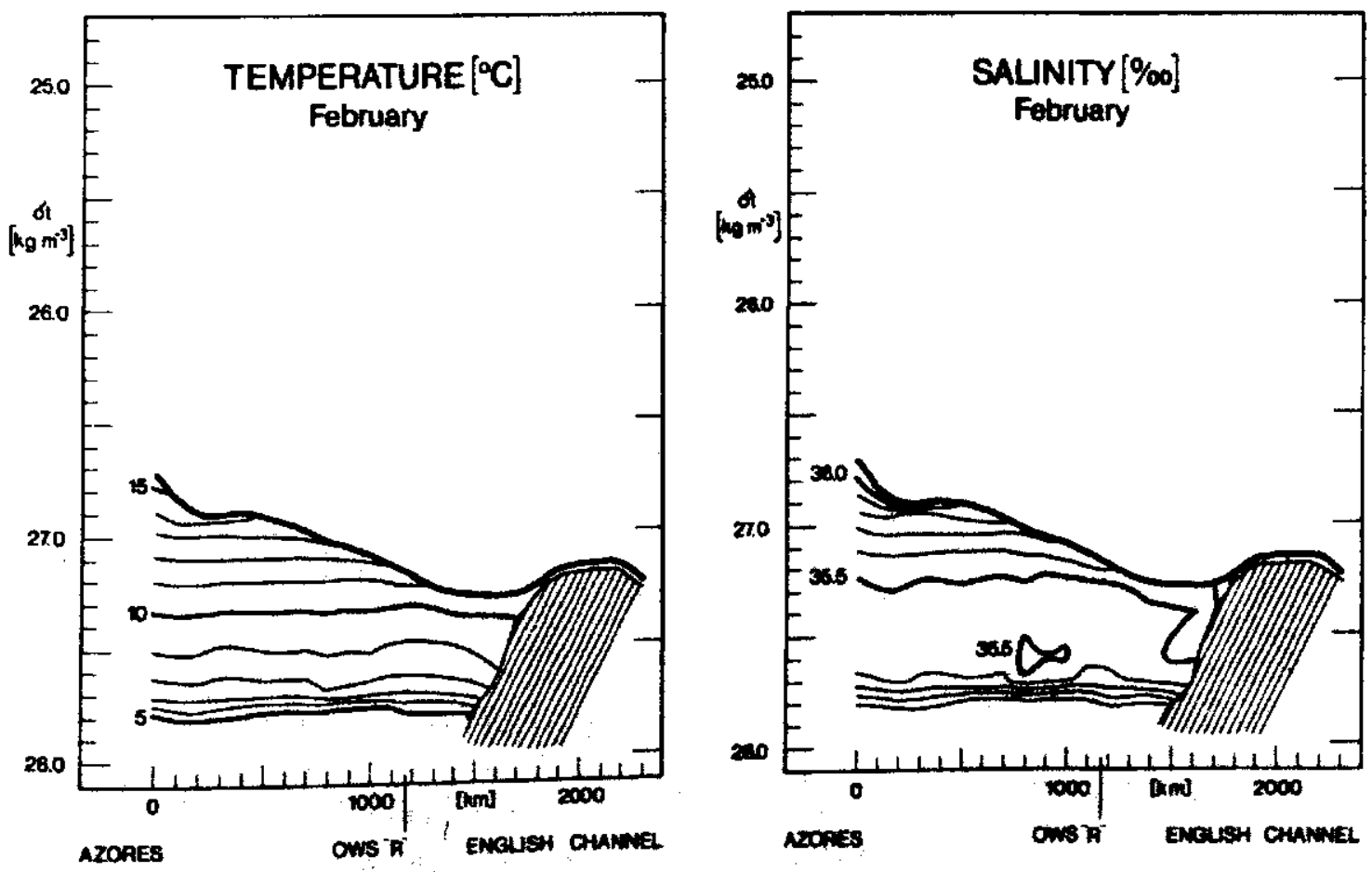


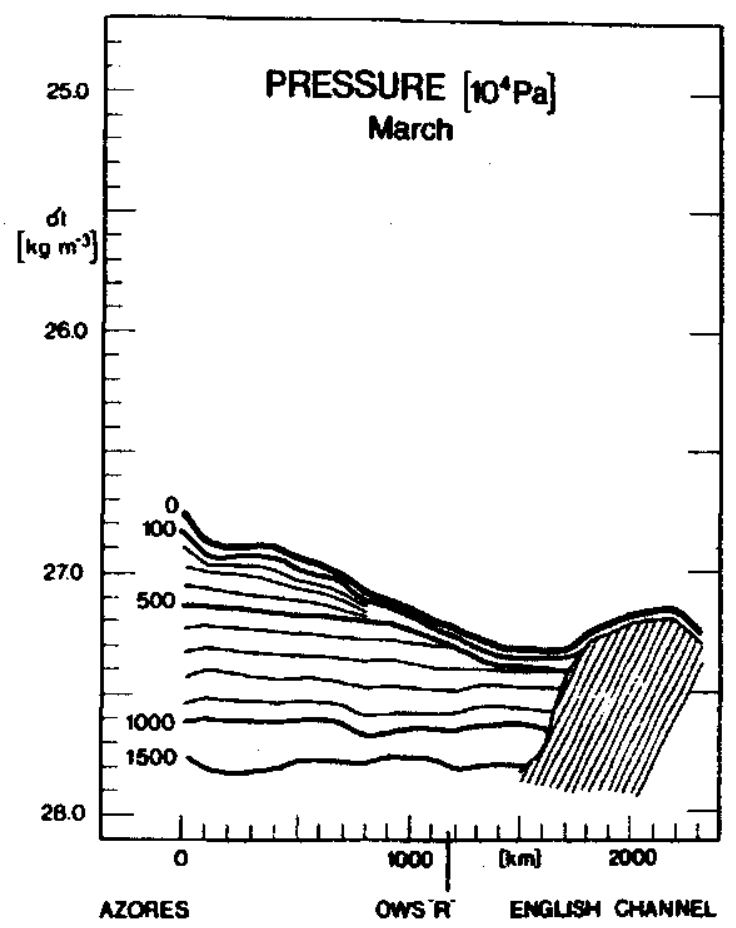

Fig. 151:

AZORES - ENGLISH CHANNEL

March
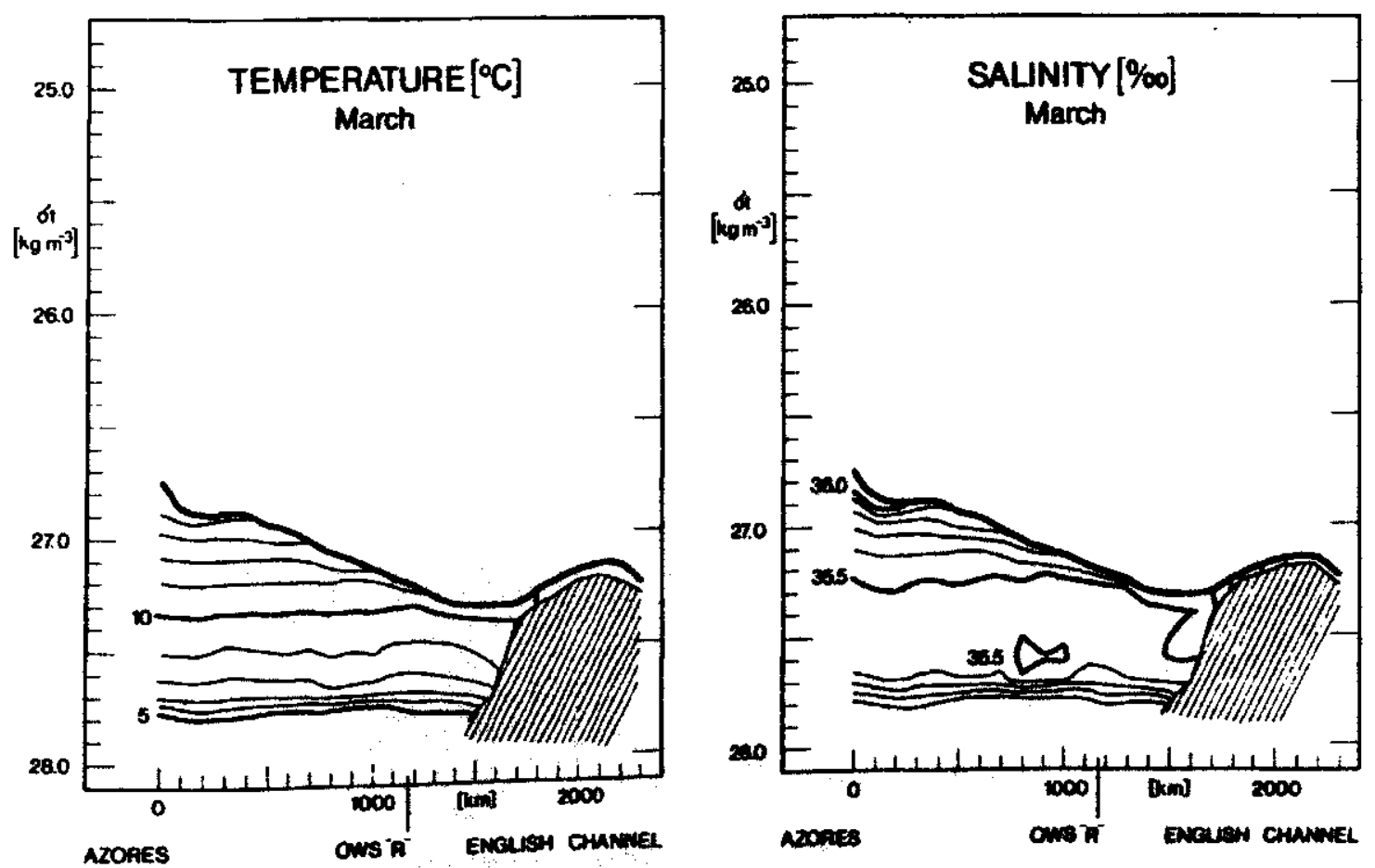


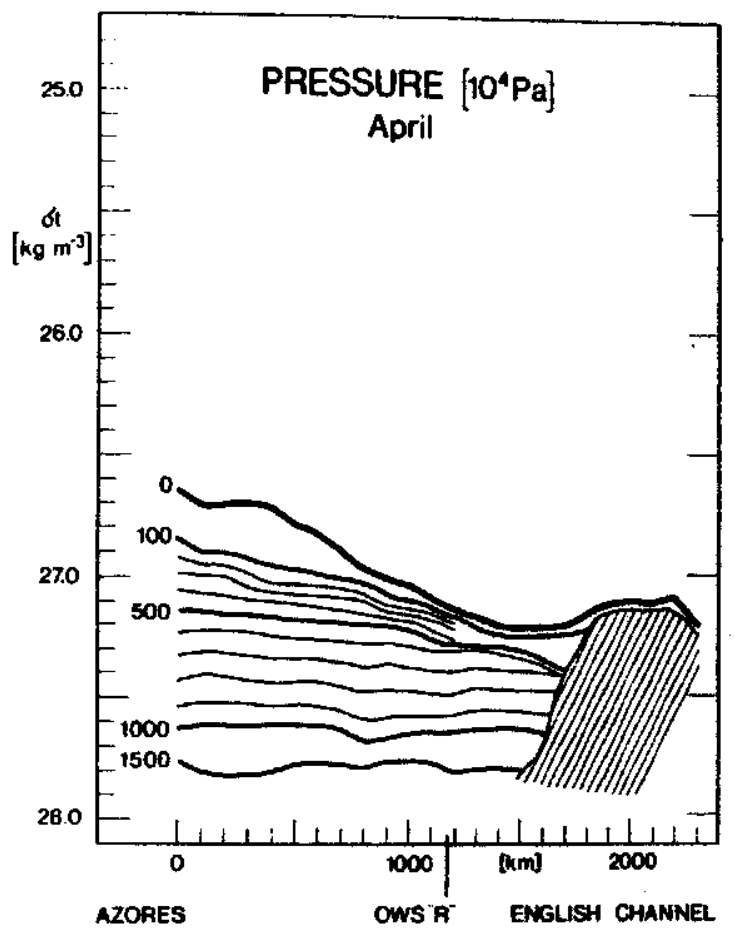

Fig. 152:

\section{AZORES - ENGLISH CHANNEL April}
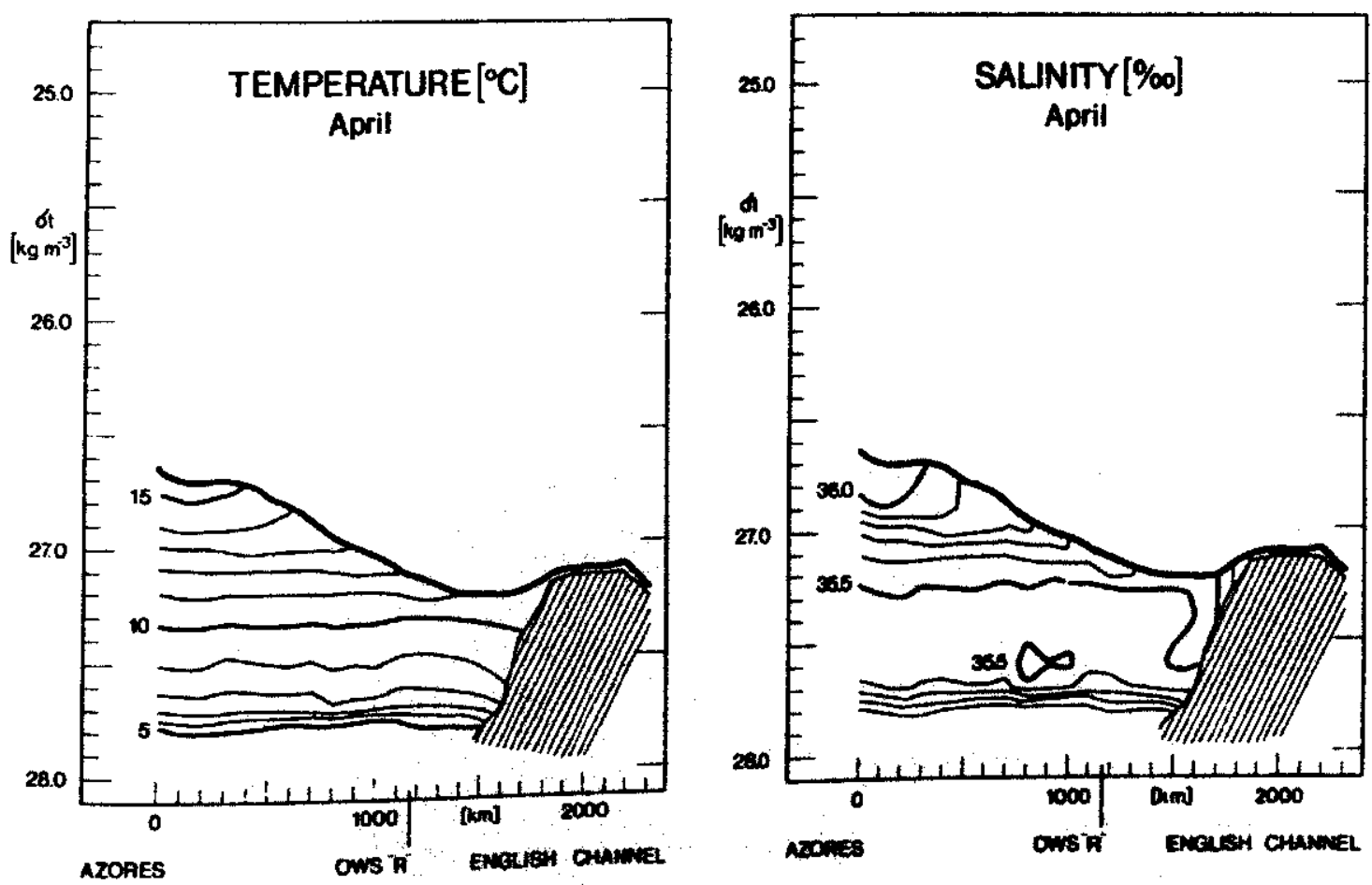


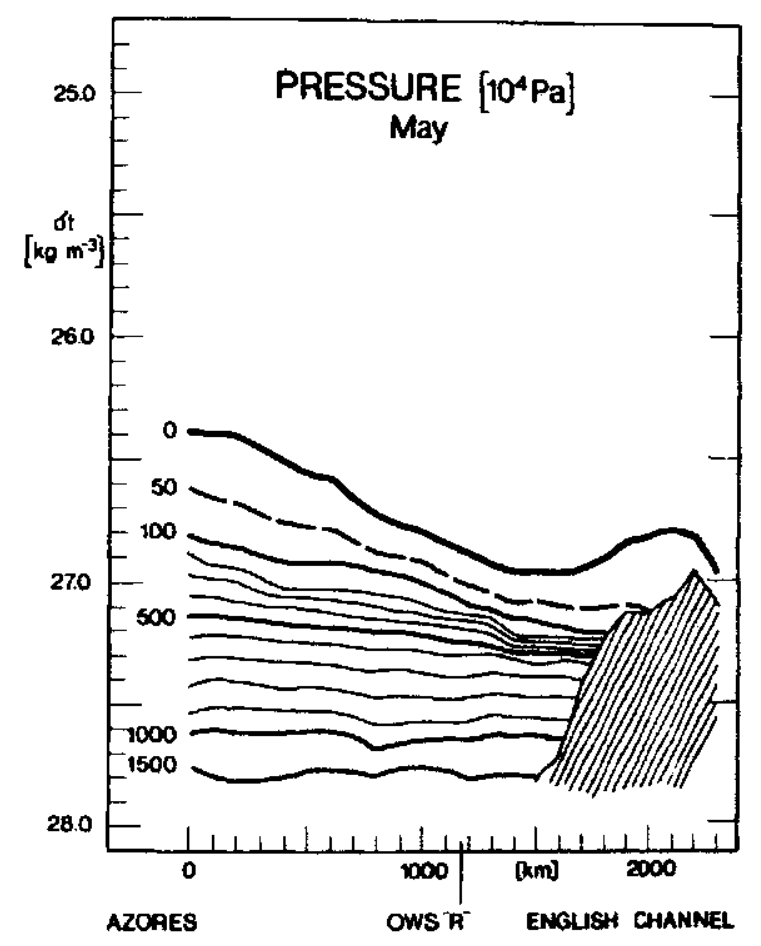

Fig. 153:

\section{AZORES - ENGLISH CHANNEL \\ May}
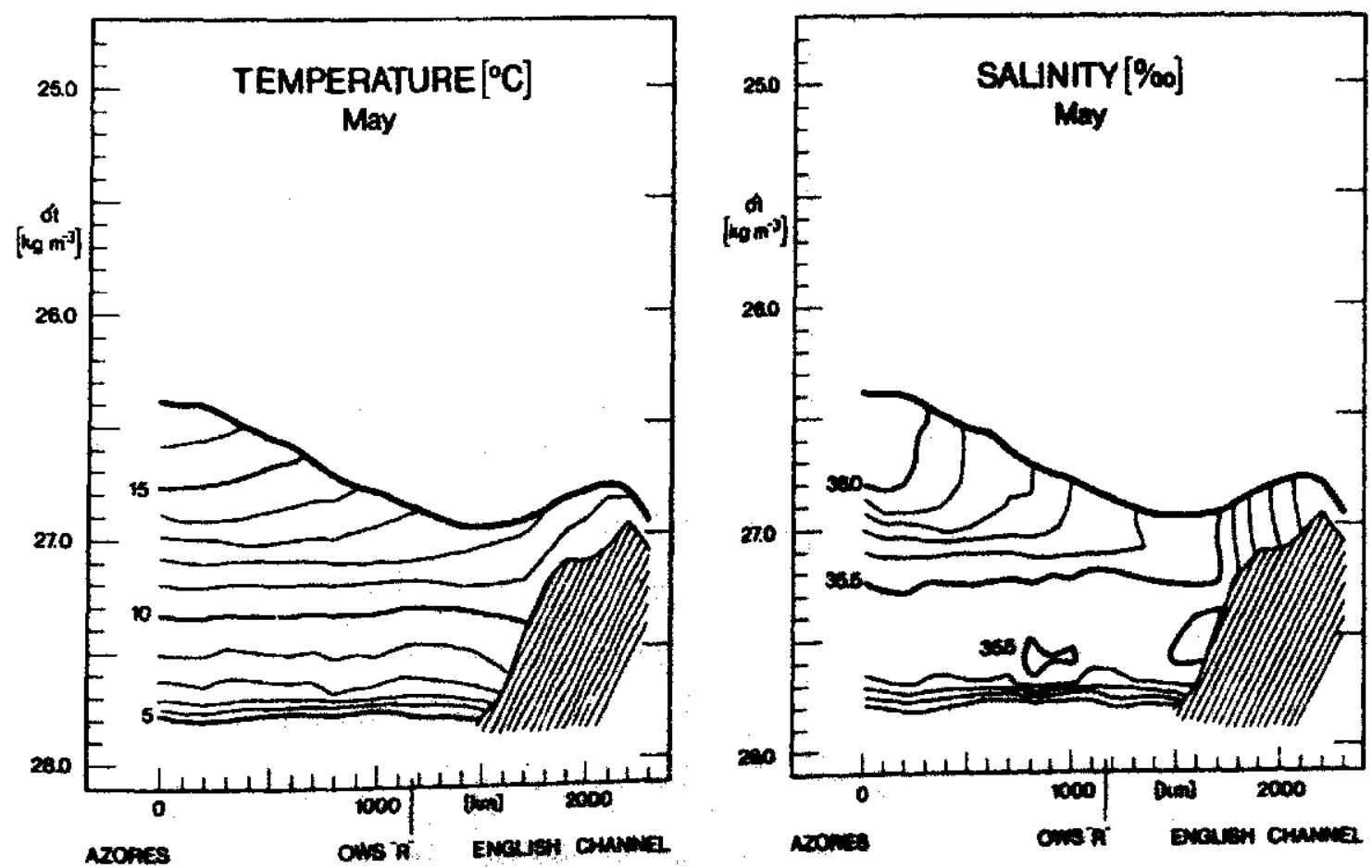


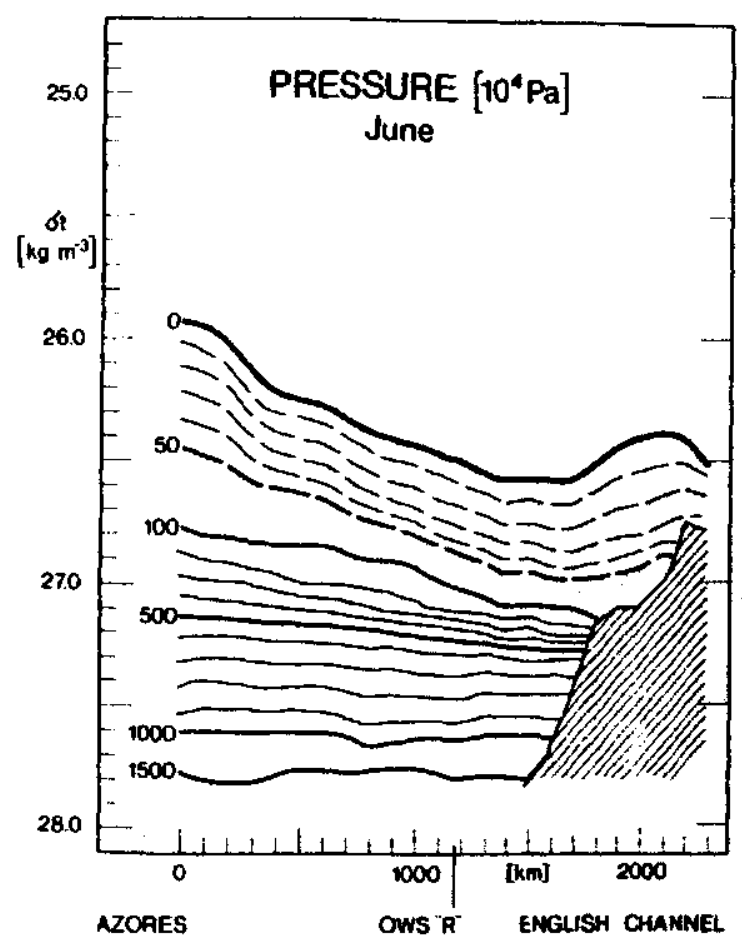

Fig. 154:

\section{AZORES - ENGLISH CHANNEL June}
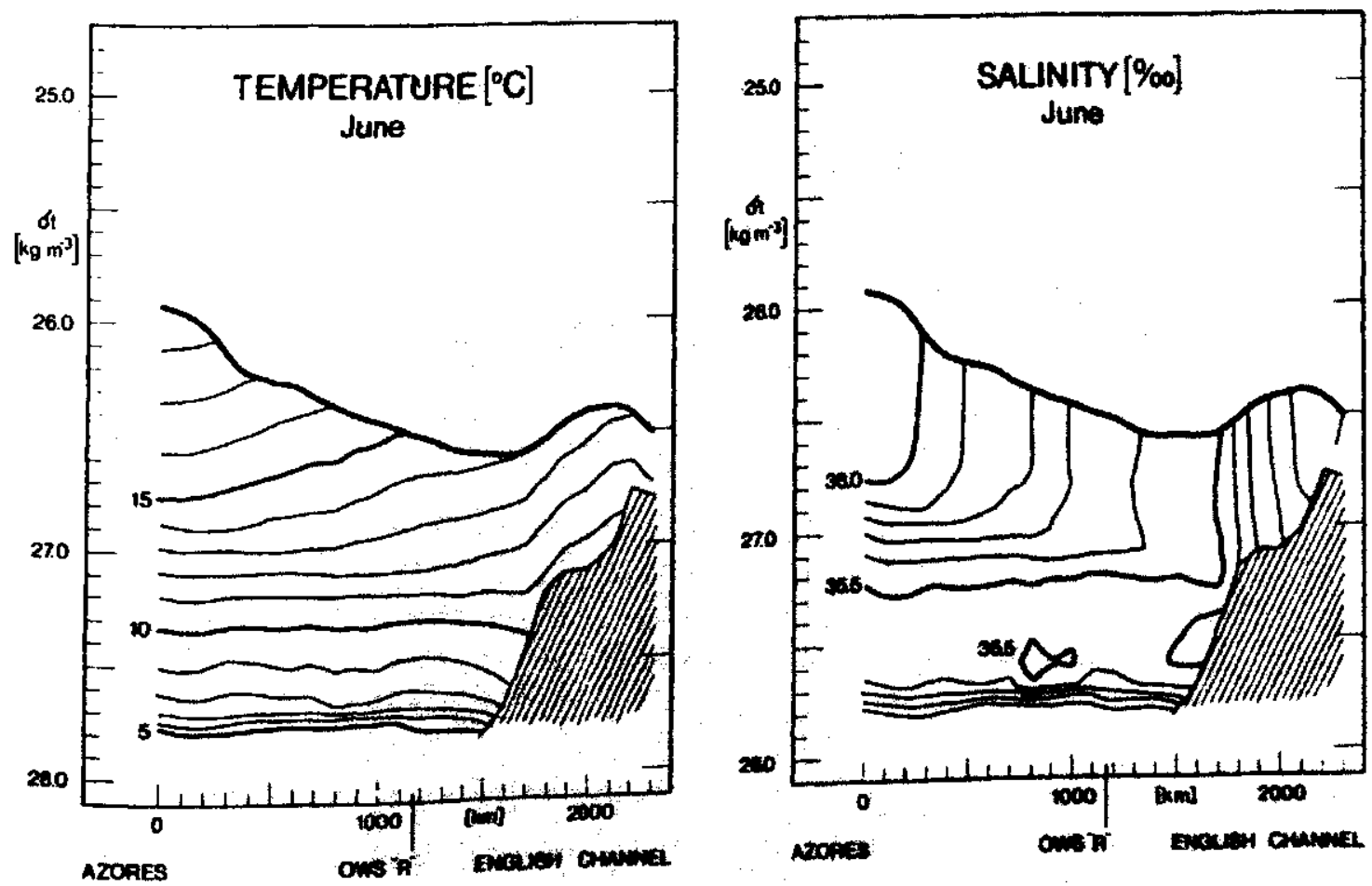


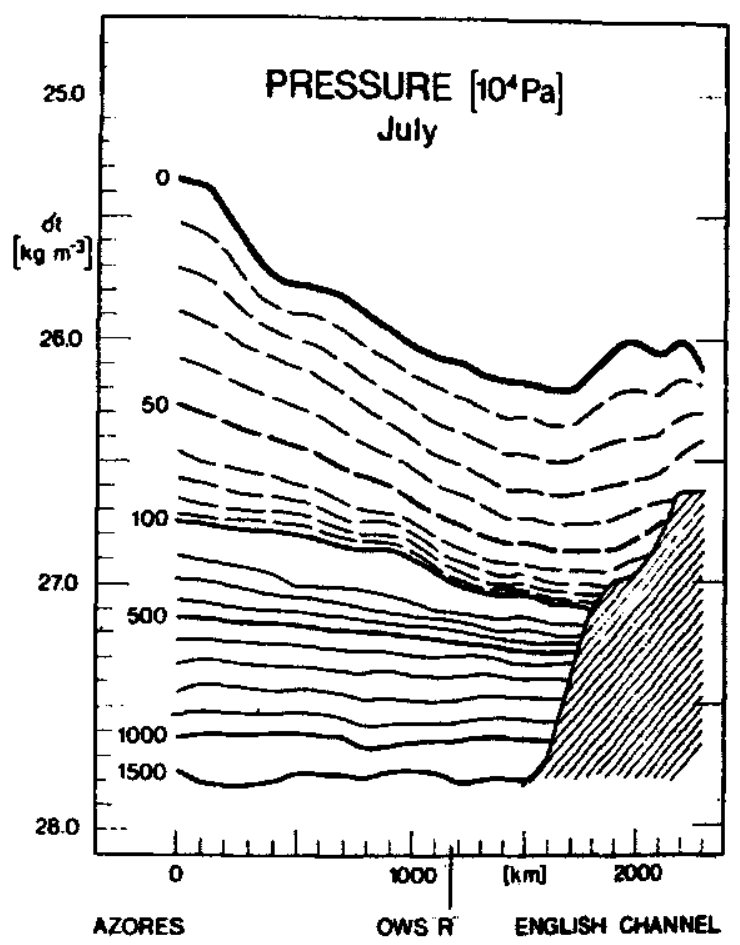

Fig. 155:

\section{AZORES - ENGLISH CHANNEL July}
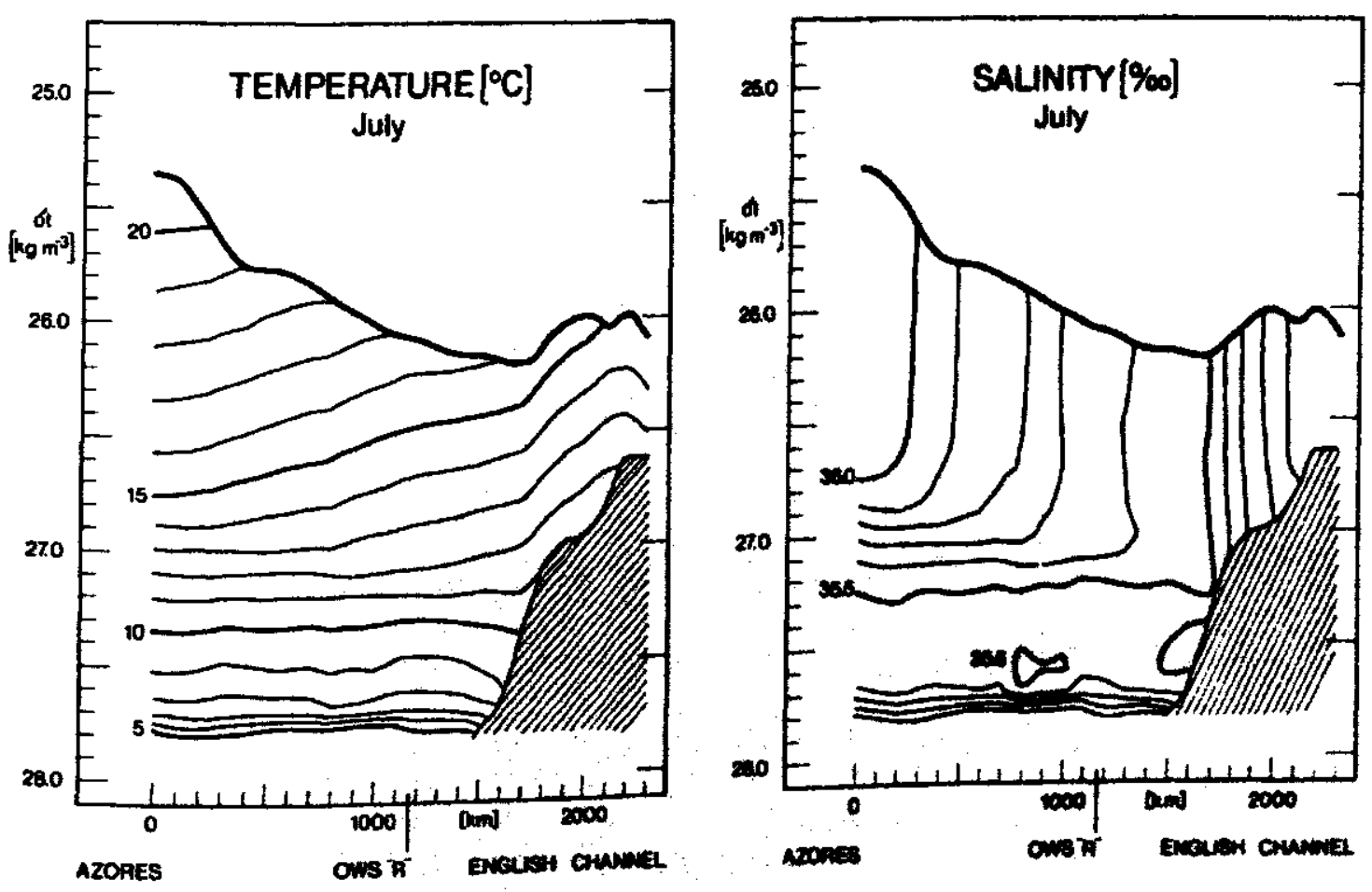


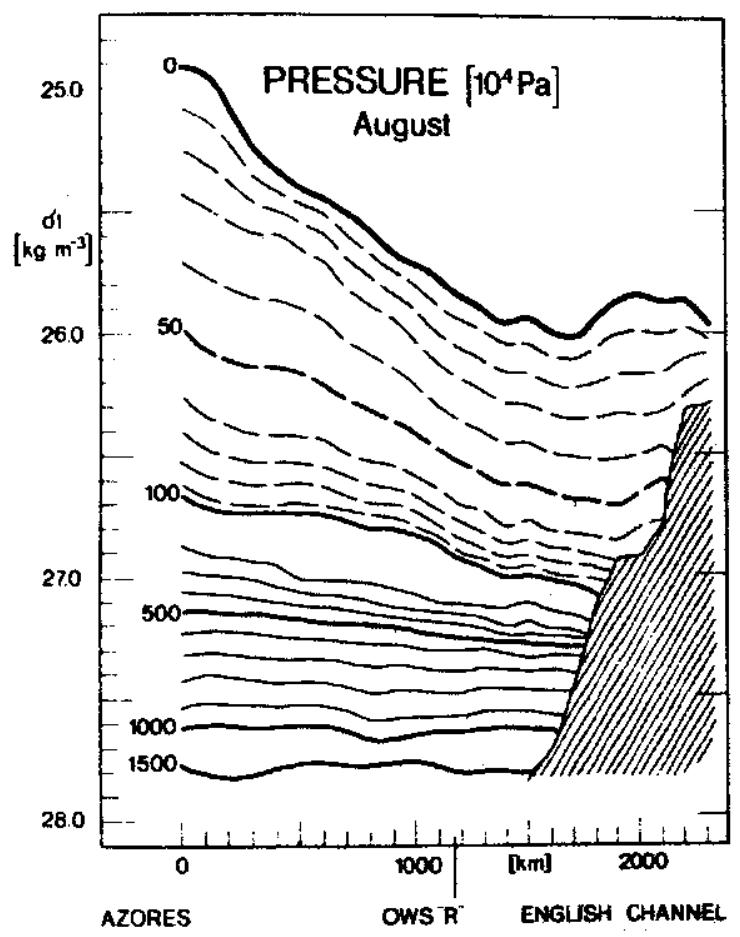

Fig. 156:

\section{AZORES - ENGLISH CHANNEL August}
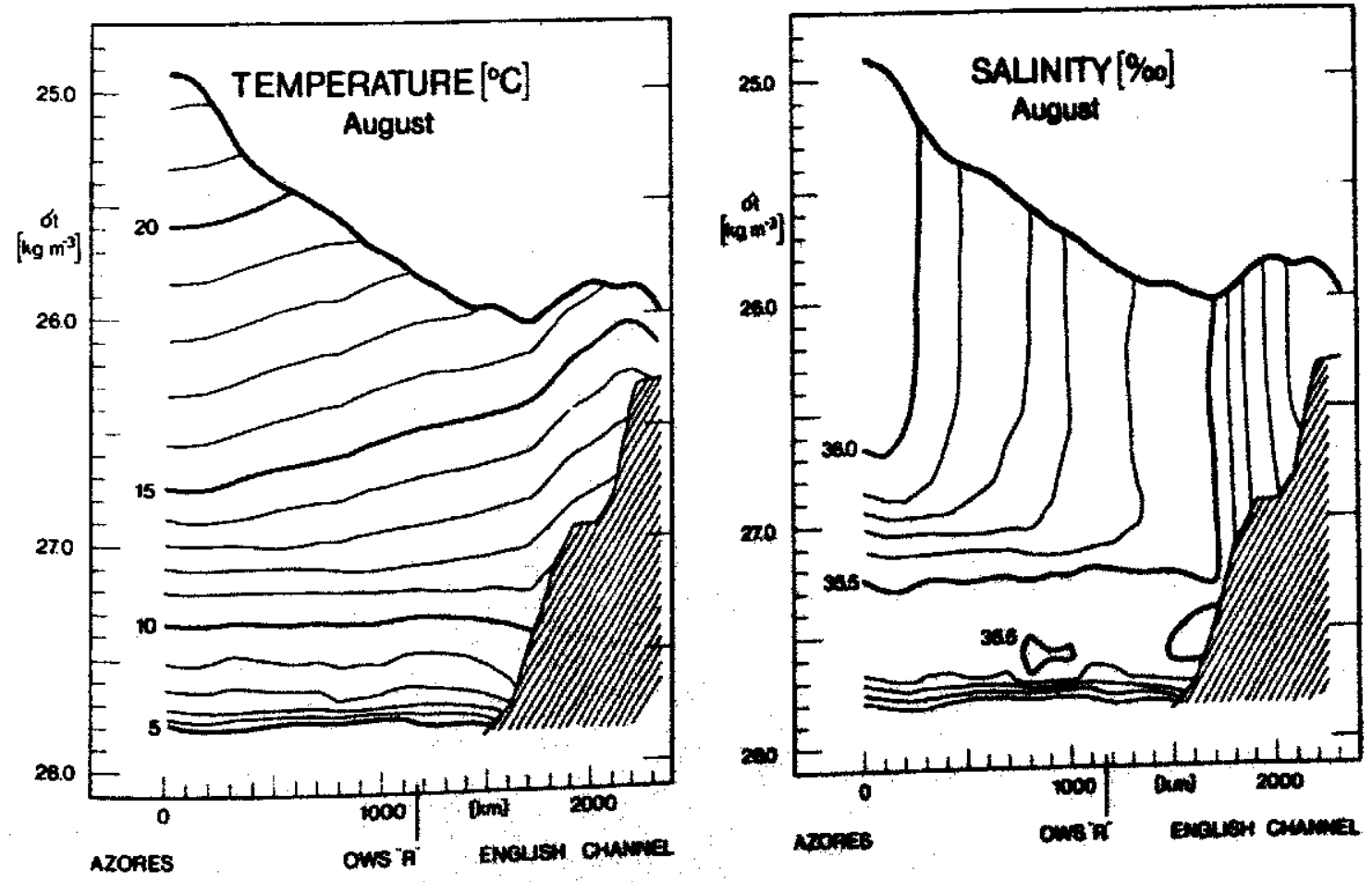


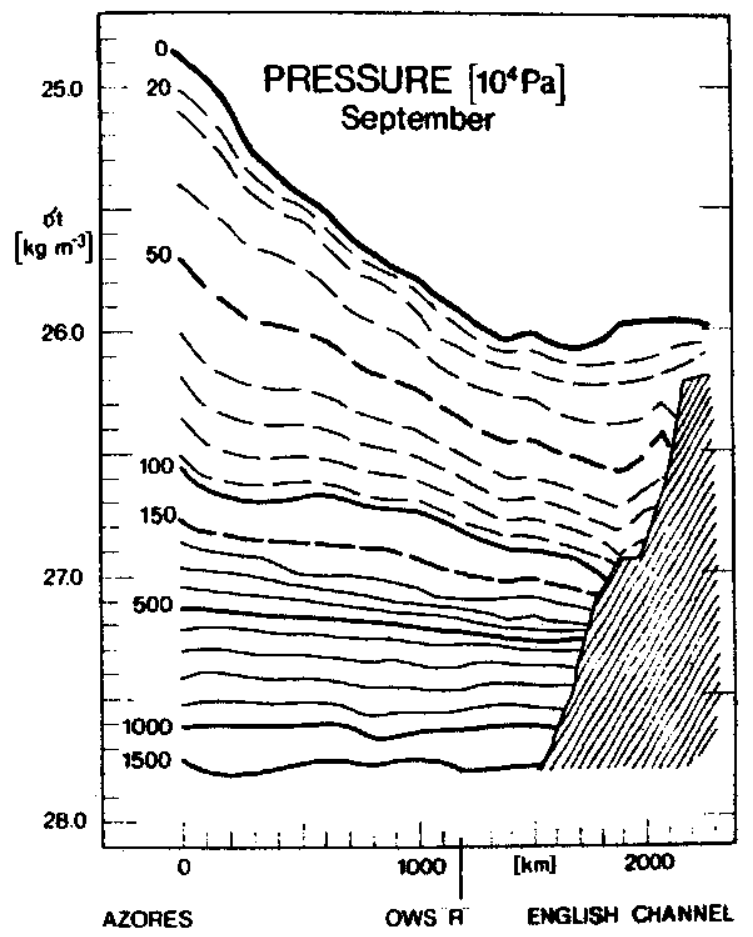

Fig. 157:

\section{AZORES - ENGLISH CHANNEL September}
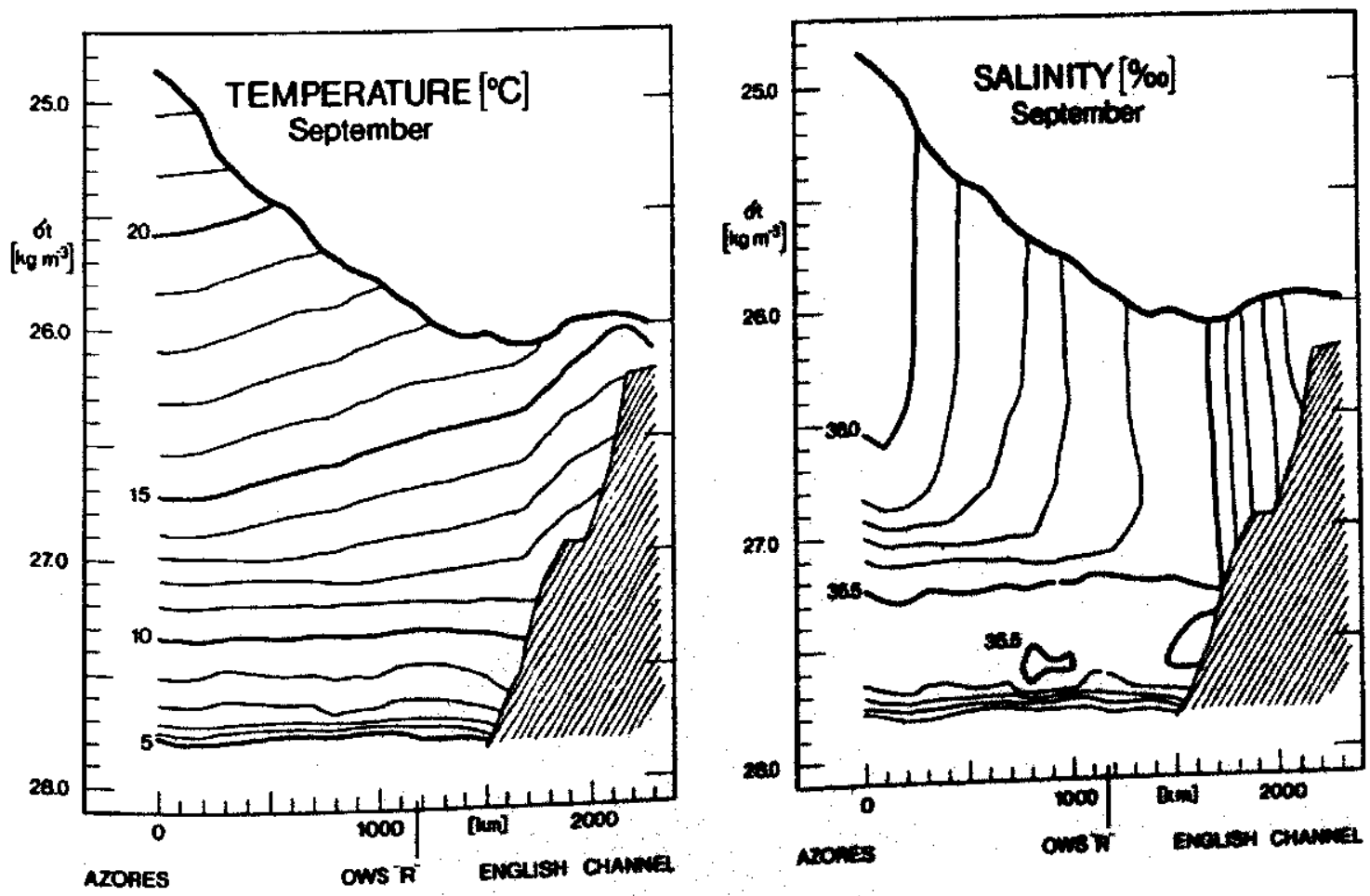


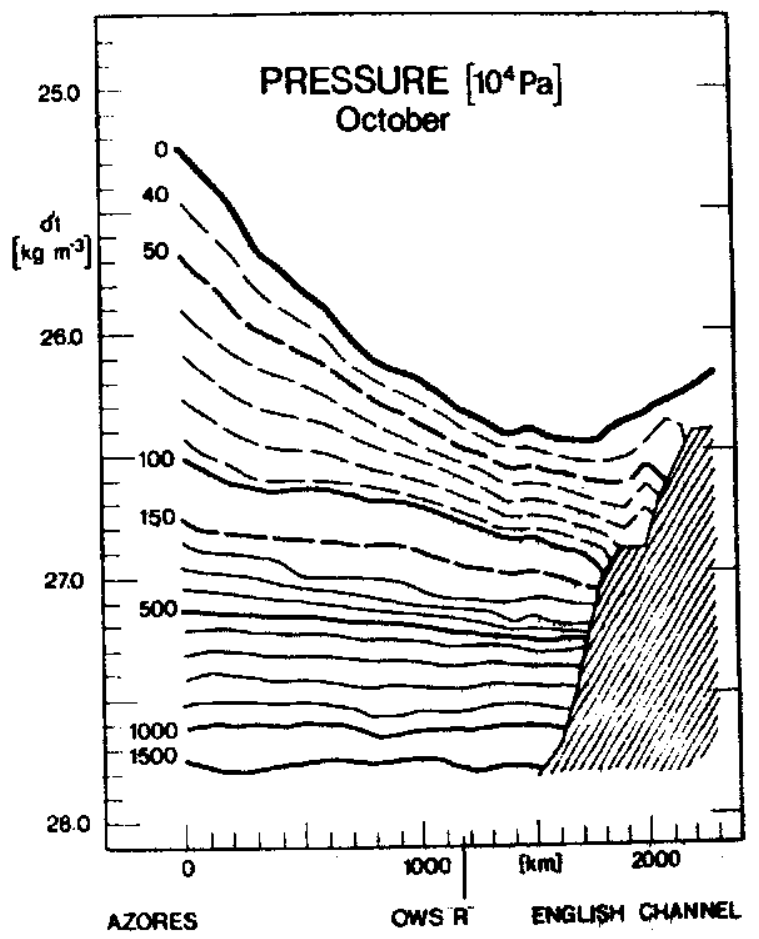

Fig. 158:

\section{AZORES - ENGLISH CHANNEL October}
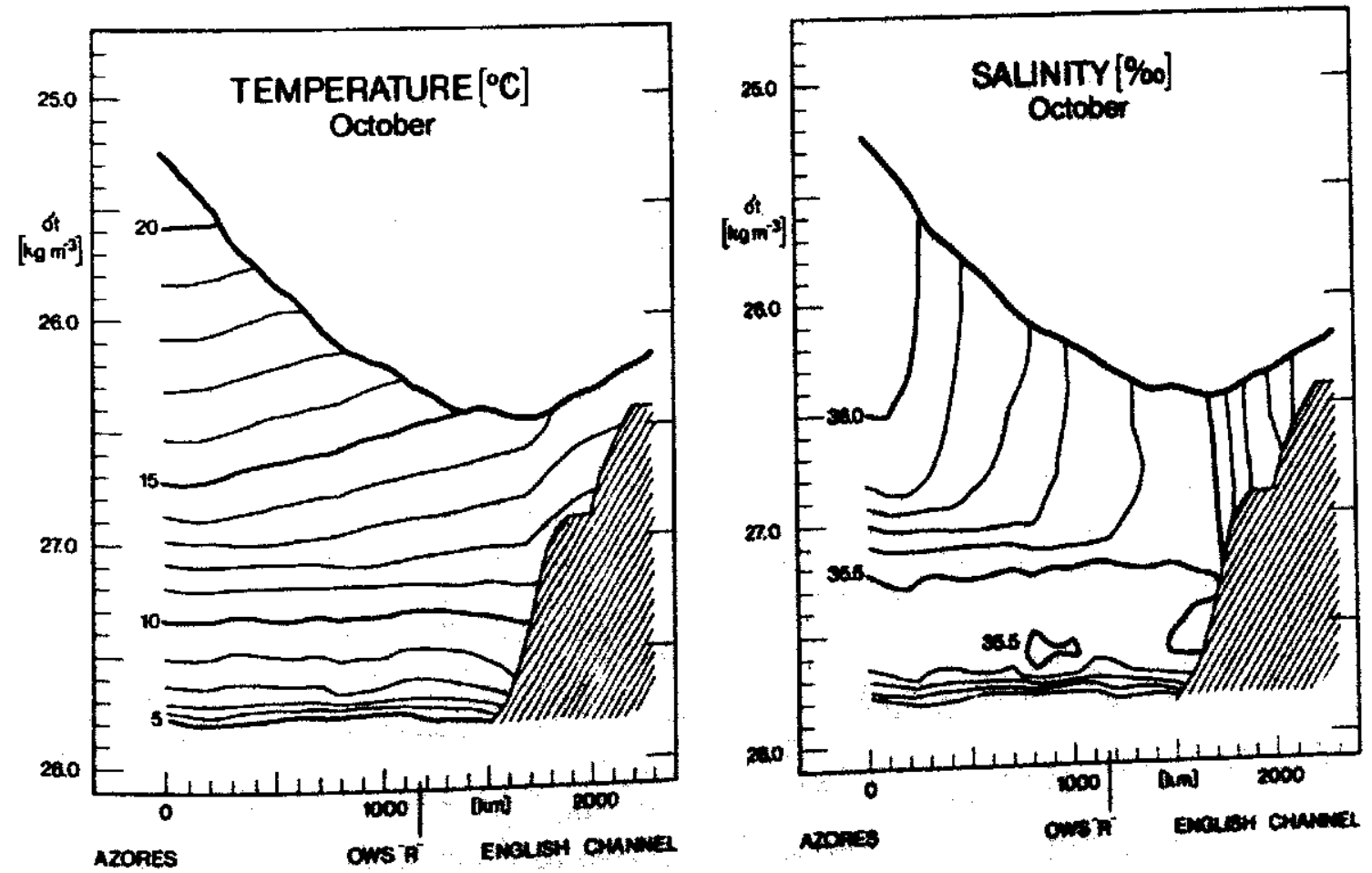


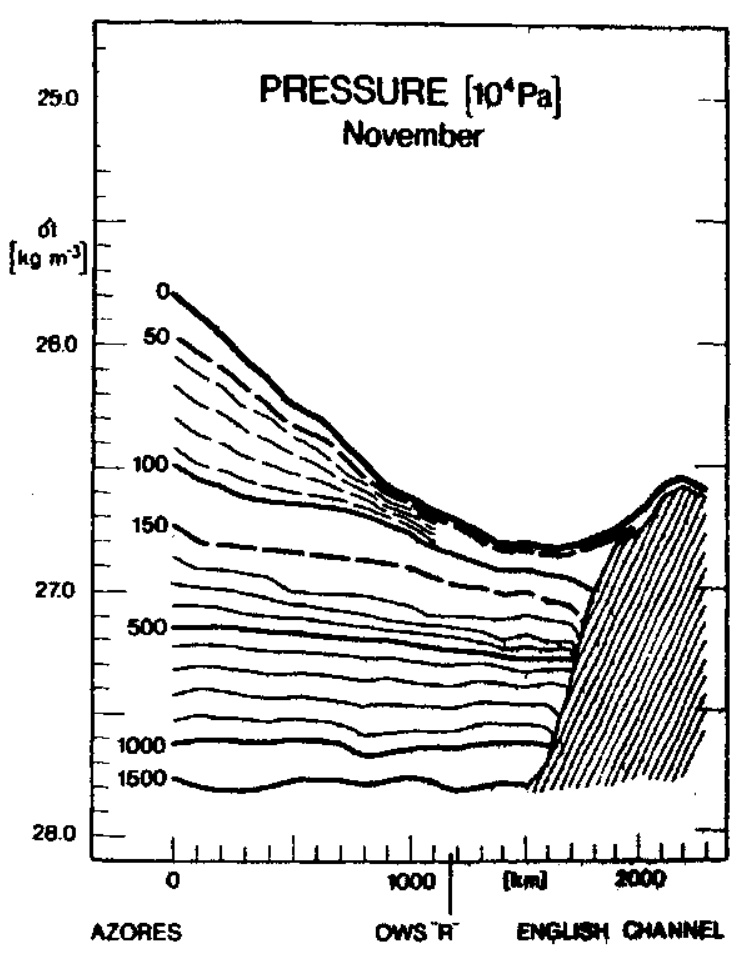

Pig. 159:

\section{AZORES - ENGLISH CHANNEL November}
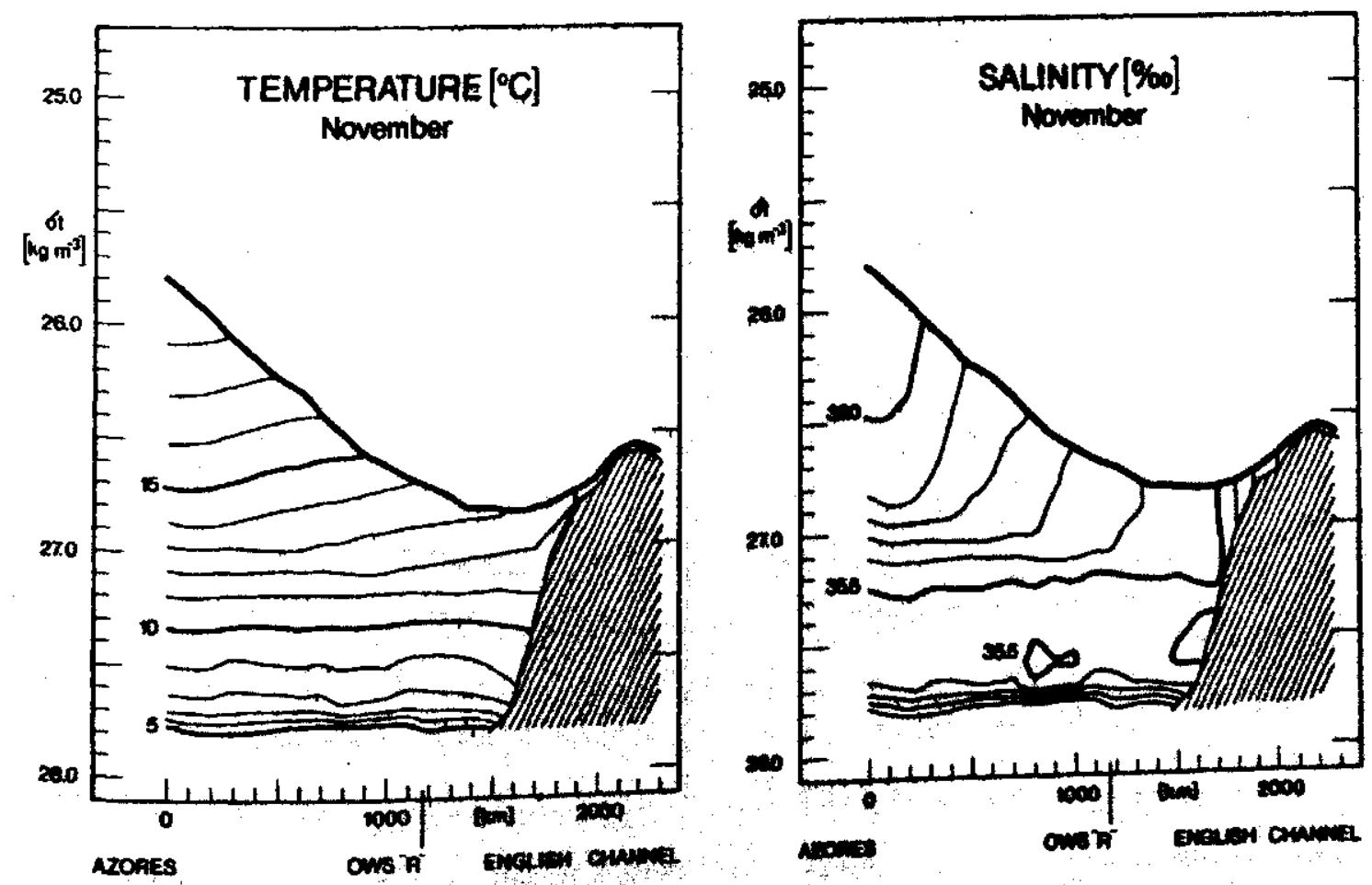


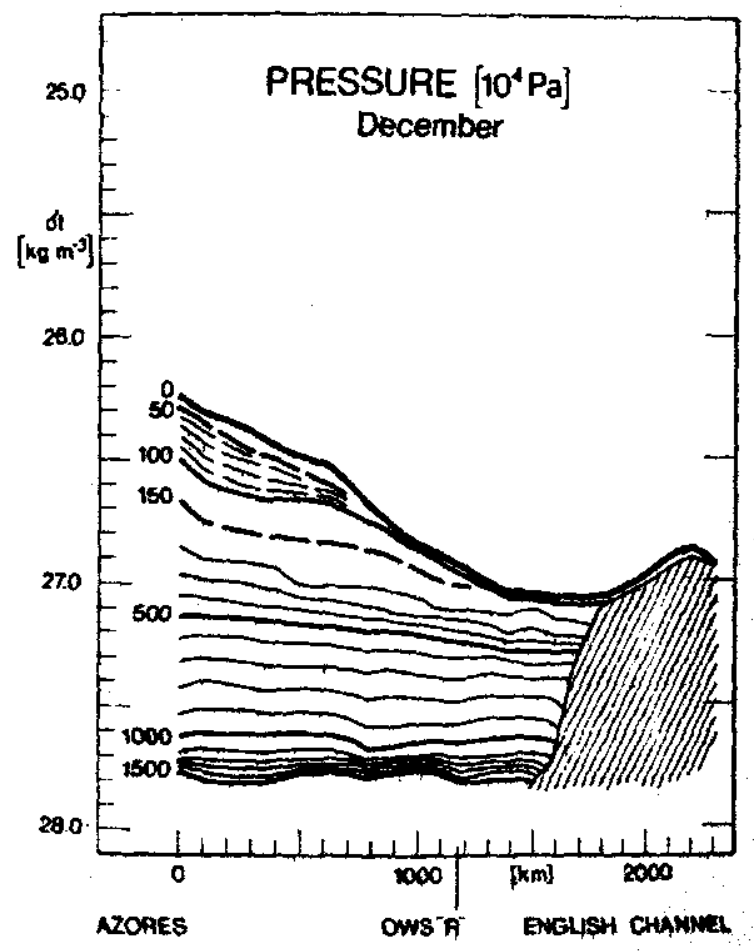

Fig. 160:

\section{AZORES - ENGLISH CHANHEL December}
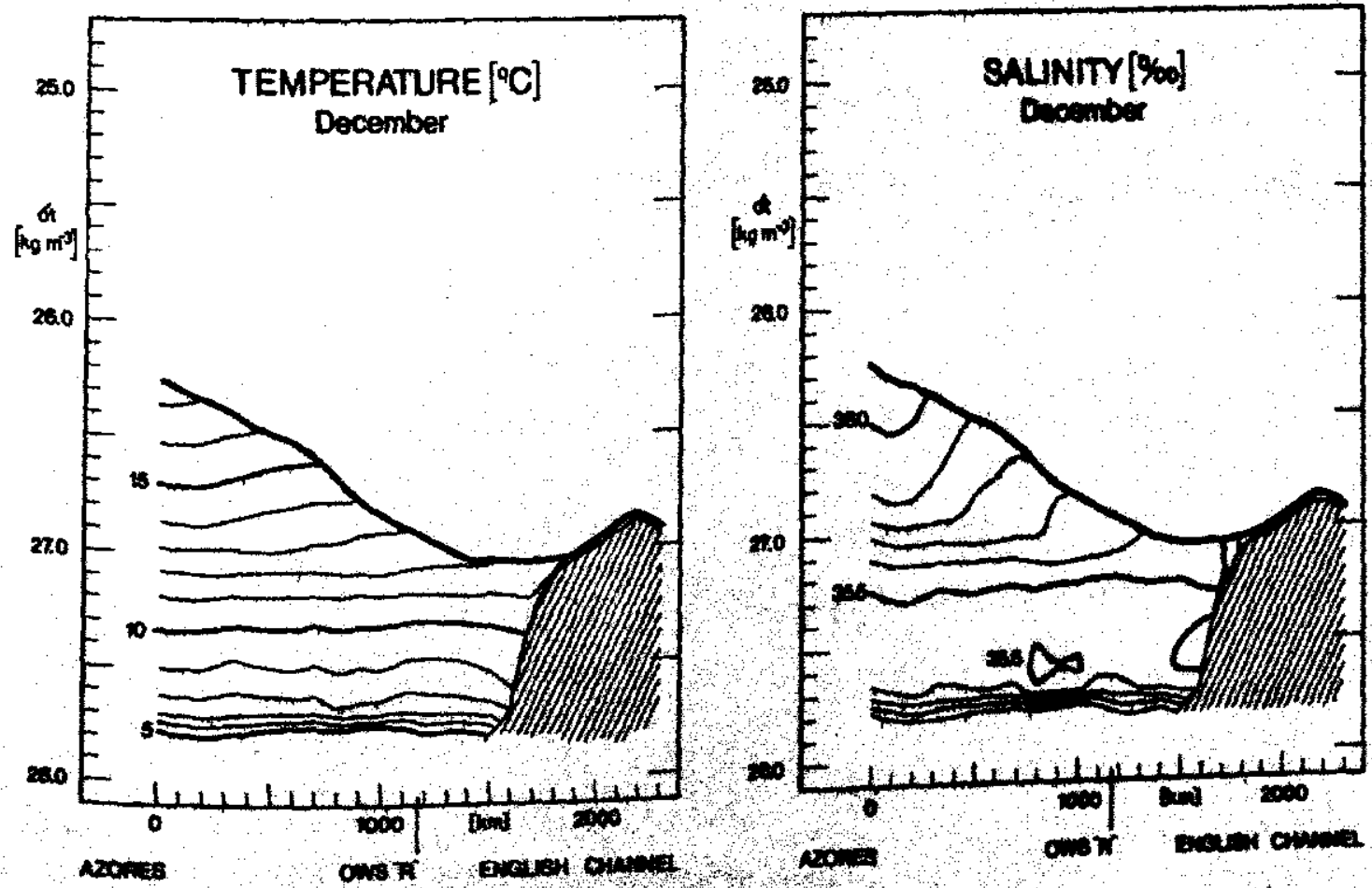